\title{
THE FIRST BRIGHT QUASAR SURVEY. II. 60 NIGHTS AND 1200 SPECTRA LATER
}

\author{
Richard L. White ${ }^{1,2}$, Robert H. BeCKeR ${ }^{2,3,4}$, Michael D. GReGG ${ }^{3,4}$, \\ Sally A. LAURENT-MUEHLEISEN ${ }^{2,3,4}$, Michael S. BROTHERTON ${ }^{2,4}$, CHRIS D. IMPEY ${ }^{5}$, \\ Catherine E. Petry ${ }^{5}$, Craig B. Foltz $Z^{5}$, Frederic H. ChafFeE $^{6}$, Gordon T. Richards ${ }^{7}$,

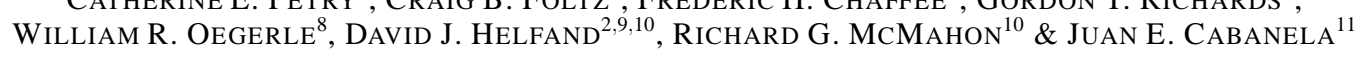 \\ Accepted for January 2000 ApJ Supplement
}

\begin{abstract}
We have used the VLA FIRST survey and the APM catalog of the POSS-I plates as the basis for constructing a new radio-selected sample of optically bright quasars. This is the first radio-selected sample that is competitive in size with current optically selected quasar surveys. Using only two basic criteria, radio-optical positional coincidence and optical morphology, quasars and BL Lacs can be identified with $60 \%$ selection efficiency; the efficiency increases to $70 \%$ for objects fainter than magnitude 17 . We show that a more sophisticated selection scheme can predict with better than $85 \%$ reliability which candidates will turn out to be quasars.

This paper presents the second installment of the FIRST Bright Quasar Survey with a catalog of 636 quasars distributed over 2682 square degrees. The quasar sample is characterized and all spectra are displayed. The FBQS detects both radio-loud and radio-quiet quasars out to a redshift $z>3$. We find a large population of objects of intermediate radio-loudness; there is no evidence in our sample for a bimodal distribution of radio characteristics. The sample includes $\sim 29$ broad absorption line quasars, both high and low ionization, and a number of new objects with remarkable optical spectra.

Subject headings: surveys — quasars: general — galaxies: active — BL Lacertae objects: general — galaxies: starburst — radio continuum: galaxies
\end{abstract}

\section{INTRODUCTION}

The VLA FIRST Bright Quasar survey (FBQS) aims to define a sample of quasars that bridges the gap between traditional radio-loud and radio-quiet objects. Given the limiting radio flux density of $1 \mathrm{mJy}$ at $1400 \mathrm{MHz}$ (Becker, White, \& Helfand 1995; hereafter BWH95), many of the optically bright quasars discovered by the FIRST survey fall near the traditional division between radio-loud and radio-quiet objects. Previous radio-selected quasar surveys have not reached deep enough to probe this regime. In a pilot study for the FBQS, Gregg et al. (1996; hereafter Paper I) developed criteria to create a candidate list based on matching the FIRST survey to the Cambridge Automated Plate Measuring Machine (APM) catalog of POSSI objects (McMahon \& Irwin 1992). Applying these criteria to the catalog of FIRST sources from the initial 2682 square degrees surveyed in the north Galactic cap (White, Becker, Helfand \& Gregg 1997; hereafter WBHG97), a candidate list of 1238 objects with extinction-corrected, recalibrated magnitudes brighter than 17.8 mag on the POSS-I $E$ plates has been assembled. We have now collected optical spectra for more than $90 \%$ of the candidates and have identified 467 new quasars in addition to the 169 that were previously known in this region.
Quasars were originally discovered through their radio emission, but only $\sim 10 \%$ of them are radio-loud ${ }^{12}$. There are now several large surveys for optically selected quasars that are under way or complete: e.g., the Palomar-Green survey (Green, Schmidt, \& Liebert 1986), the Large Bright Quasar Survey (Hewett, Foltz \& Chaffee 1995; hereafter LBQS), the Edinburgh Quasar Survey (Goldschmidt et al. 1992), and the Hamburg/ESO survey (Wisotzki et al. 1996, Hagen, Engels \& Reimers 1999). The FBQS survey will produce a sample of radio-selected quasar spectra that is comparable in size and quality to the largest existing optical surveys. In fact, the catalog published in this paper already contains more $z>0.2$ quasars brighter than $B=18$ than does the LBQS (340 for the FBQS vs. 319 for the LBQS) because even though radio quasars are rarer, the FBQS covers a substantially larger sky area than the LBQS (2682 $\mathrm{deg}^{2}$ vs. $454 \mathrm{deg}^{2}$ for the LBQS.)

In this paper we present this new radio-selected sample of quasars. We discuss the criteria used to define the FBQS sample, as well as a new technique that could be used to make even more efficient samples (\$2). We describe the optical spectroscopy that was carried out (\$3), present the results of the spectroscopy including spectroscopic classifications for all ob-

\footnotetext{
${ }^{1}$ Space Telescope Science Institute, 3700 San Martin Dr., Baltimore, MD 21218, rlw@ stsci.edu

${ }^{2}$ Visiting Astronomer, Kitt Peak National Observatory, National Optical Astronomy Observatory

${ }^{3}$ Physics Dept., University of California-Davis

${ }^{4}$ IGPP/Lawrence Livermore National Laboratory

${ }^{5}$ Steward Observatory, U. Arizona

${ }^{6}$ W. M. Keck Observatory

${ }^{7}$ Astronomy \& Astrophysics Center, U. Chicago

${ }^{8}$ Dept. of Physics \& Astronomy, Johns Hopkins University

${ }^{9}$ Astronomy Dept., Columbia University

${ }^{10}$ Institute of Astronomy, Cambridge University

${ }^{11}$ Dept. of Astronomy, U. Minnesota

${ }^{12}$ We define radio-loud quasars as those with a radio-loudness parameter $R^{*}$ greater than 10 (Stocke et al. 1992; see $\$ 2.5$ for further discussion.) 


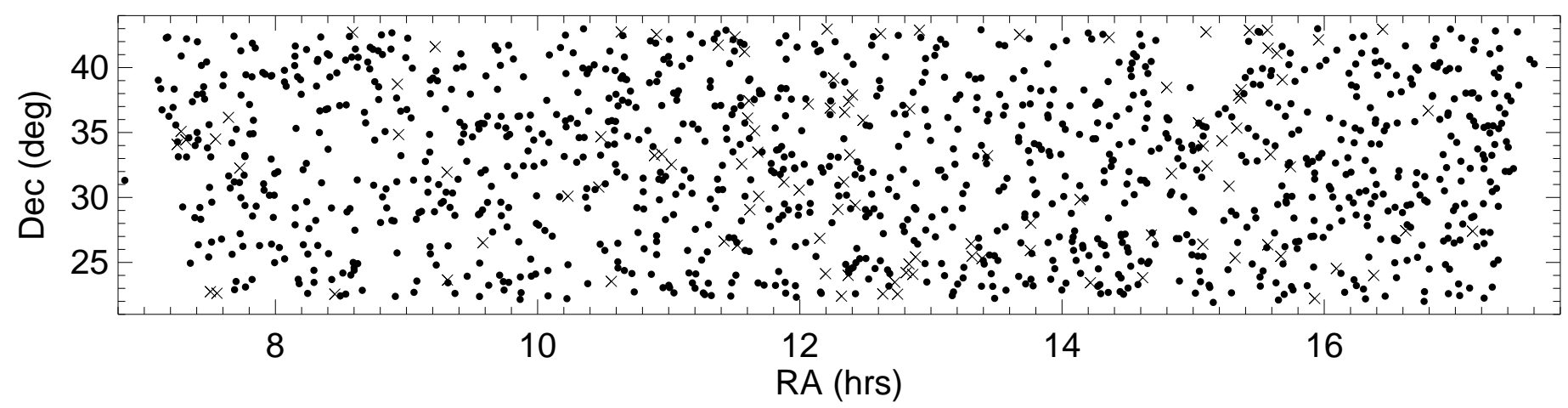

FIG. 1.- Distribution of the $1238 \mathrm{FBQS}$ candidates on the sky. Dots are the 1130 objects identified through spectra; x's mark the 108 objects without spectra.

jects ( $\$ 4)$, and briefly discuss the results ( $\$ 5)$. Detailed analysis of the FBQS sample, including the spectral properties of the new quasars, will be deferred to other papers; here our focus is on defining the sample and on presenting the basic data upon which subsequent work will be based.

\section{THE SAMPLE}

The primary catalog for the FBQS is the VLA FIRST survey which, as of July 1999, covers $\sim 6000 \mathrm{deg}^{2}$ (BWH95, WBHG97; see also the FIRST home page at http://sundog.stsci.edu). However, at this time the vast majority of optical spectroscopy has been restricted to objects drawn from the smaller area covered by the 1997Apr24 version of the FIRST northern Galactic cap catalog. For the purposes of this paper, we will restrict the discussion to candidates in the north Galactic cap between declinations of +22 and +43 degrees, with Right Ascensions ranging from approximately $\sim 7$ to $\sim 17$ hours; the area covered is 2682 square degrees. The spatial distribution of the candidates included in the paper is shown in Figure 1. The selection criteria for membership in the sample are:

- The radio and optical positions must coincide to better than 1.2 arcsec.

- The recalibrated, extinction-corrected optical magnitude on the POSS-I red plate $E \leq 17.8$. (The $O$ magnitude roughly corresponds to $B$ and $E$ to $R$ or to Gunn $r$.)

- The optical morphology of the object must be stellar on at least one of the two POSS-I plates.

- The POSS-I color must be bluer than $O-E=2$.

We avoid as far as possible any restriction on the radio properties of sources in the sample, other than requiring them to be in the FIRST catalog, which has its own selection effects. These criteria were based on the analysis presented in Paper I and have been found to be quite liberal, excluding very few potential quasars. The criteria are discussed in detail below.

\subsection{Radio-Optical Positional Coincidence}

We require that the radio and optical sources be separated by no more than 1.2 arcsec. A discussion of the astrometric accuracy that allows for such a tight constraint is found in WBHG97. The FIRST radio positions have been used to correct the APM positions for POSS-I plate distortions before comparing the radio and optical positions (McMahon et al. 1999). In general, the required close agreement in position excludes quasars that do not have at least a weak core radio component, so the candidate list is biased against completely lobe-dominated radio sources. The distribution of radio-optical separations for confirmed quasars is strongly peaked and falls rapidly beyond 0.5 arcsec (see Fig. 2a). Only $5(0.8 \%)$ of our 636 quasars have separations between 1.1 and 1.2 arcsec, even though that annulus contains $16 \%$ of the area searched. Nonetheless, this criterion certainly excludes some quasars that are detected by the FIRST survey. Figure 2(b) shows the fraction of optical candidates that are found to be quasars as a function of separation; it declines steadily from $\sim 80 \%$ near 0 arcsec to $\sim 20 \%$ at 1.2 arcsec. If we make the conservative assumption that the quasar fraction does not decline further, we estimate that there are $<10$ additional quasars with separations between 1.2 and 1.5 arcsec that are excluded from the sample by the separation criterion.

\subsection{E Magnitude}

The original candidate list was limited to optical counterparts that are brighter than $17.5 \mathrm{mag}$ on the POSS-I $E$ plate as measured by the APM scans. In fact, the APM $E$ magnitude typically overestimates the actual brightness by $\sim 0.3 \pm 0.3 \mathrm{mag}$, so the original candidate list went slightly deeper than intended and was not uniform over the survey area. In an attempt to improve the photometric accuracy and uniformity of the sample, the APM magnitudes were subsequently recalibrated plate-byplate (McMahon et al. 1999) using magnitudes from the Minnesota Automated Plate Scanner POSS-I catalog (APS ${ }^{13}$, Pennington et al. 1993), which are more uniform than the APM magnitudes because they were calibrated on a plate-by-plate basis, and a new limit of 17.8 in $E$ was used to redefine the complete sample. We preferred not simply to substitute the APS catalog for the APM because the APS sky coverage is incomplete in the FIRST region and because the APS catalog excludes objects detected on only one of the POSS-I plates, which dramatically reduces the fraction of FIRST sources that have optical identifications since most radio source counterparts are near the plate limit and so appear on only one plate. The latter consideration is not important for the FBQS, but is a disadvantage for most other optical projects.

After the APS recalibration, we find that the APM magnitudes are accurate to better than 0.2 magnitudes rms in both $\mathrm{O}$ and $\mathrm{E}$ (McMahon et al. 1999). This accuracy was determined

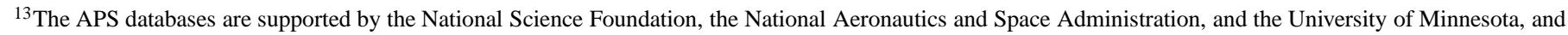
are available at http://aps .umn . edu/.
} 


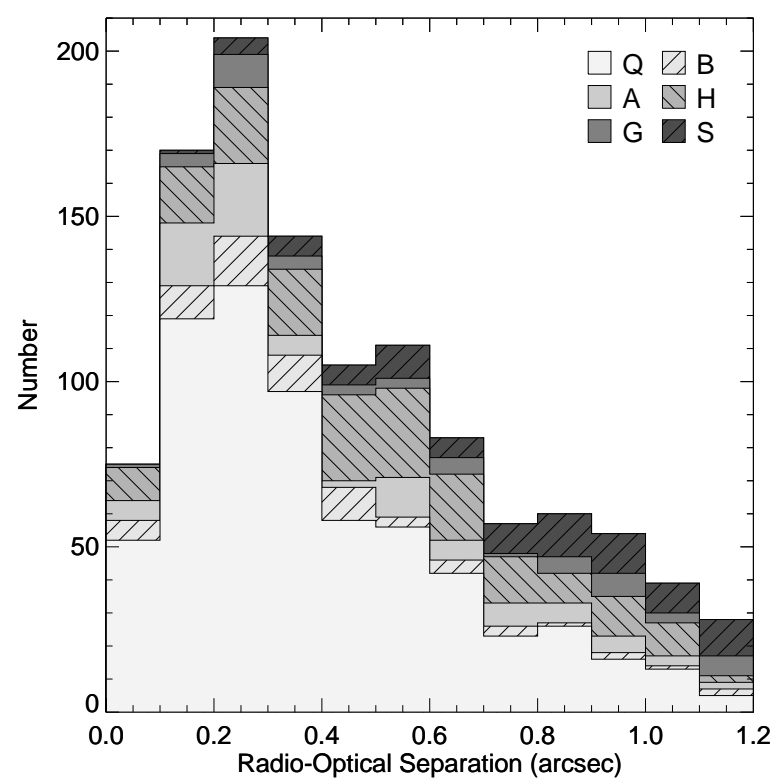

(a)

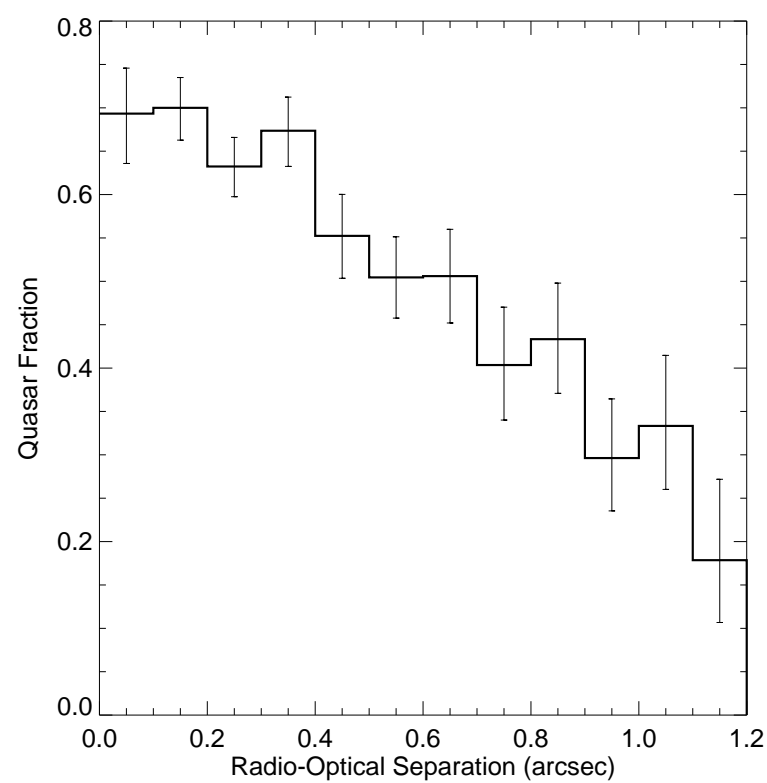

(b)

FIG. 2.- (a) Histogram of separations for FBQS candidates identified as quasars (Q), BL Lacs (B), narrow-line AGN (A), H II/star-forming galaxies (H),

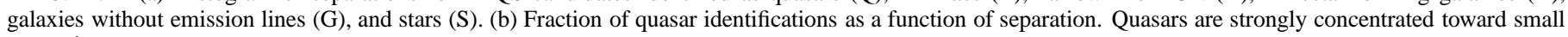
separations.

by comparing the magnitudes of objects appearing in the narrow overlap regions at the edges of the POSS-I plates; since the photometric errors are probably worst at the plate edges, for most objects the errors may be even smaller.

As a result of the E magnitude adjustments, the sample was augmented by approximately 100 candidate quasars. Furthermore, there are instances of sources in the APS catalog that are not detected on one or both plates in the APM catalog (the opposite also occurs.) The most common reason for such missing sources is that close objects are blended into a single merged entry. We added 26 such APS-supplement objects to our candidate list; these objects are noted in the comments. Finally, to improve the uniformity of the sample with Galactic latitude, an extinction correction was computed for each candidate object using the $E(B-V)$ map of Schlegel, Finkbeiner \& Davis 1998 with $A(E)=2.7 E(B-V)$ and $A(O)=4.4 E(B-V)$ (estimated from the $E$ and $O$ bandpasses given in Minkowski \& Abell 1963.) These corrections are usually quite small — the median values are $A(E)=0.058$ and $A(O)=0.094$ - but can be significant at the high and low RA edges of the survey (see Fig. 3). We keep all objects that satisfy $E \leq 17.8$ and the $O-E$ color cut described below using the extinction-corrected magnitudes; this adds an additional $\sim 90$ candidates to the sample.

The extinction-corrected magnitude distribution of identified candidates is shown in Figure 4(a) for various classifications. (The definitions adopted for these object classifications are given in \$4; briefly, a "quasar" is any object with broad emission lines.) The efficiency of finding quasars is a strong function of magnitude. Figure 4(b) shows the fraction of candidate objects that are quasars as a function of $E$ magnitude. Less than $5 \%$ of the objects brighter than $E=14$ mag are quasars (most such objects are brighter than $E=13$ and so do not appear in Fig. 4); this grows to $70 \%$ for candidates fainter than $E=17$ mag. Most of the brightest nonquasars are galaxies that the APM has misclassified as stellar.
The very steep increase in the number of quasars with increasing magnitude introduces a strong Malmquist bias into this sample (and other samples of bright quasars.) There is a large pool of quasars just below the magnitude threshold, so the sample is certain to include variable objects that happened to be unusually bright when the POSS-I plates were taken; it will also include quasars with measurement and/or calibration errors that made them look brighter than they really are. When analyzing properties of the sample, readers are cautioned to consider this bias. See our study of the variability of a subset of the FBQS quasars for further discussion of this issue (Helfand et al. 1999b).

\subsection{Optical Morphology}

Since we are searching for quasars, we require all candidates to be classified by the APM as stellar on at least one POSS-I plate. The APM star/galaxy separator is not infallible, however. Approximately $14 \%$ of the quasars found in the FBQS are classified as nonstellar on one of the two POSS-I plates. Since we admit Seyfert galaxies into our quasar sample, some of these nonstellar classifications may be correct. Fully $75 \%$ of the objects classified as stellar on both plates turned out to be quasars. In comparison, only $25 \%$ of the objects classified as stellar on only one plate proved to be quasars.

Nonetheless, it is likely that some quasars are being lost to the sample because they are being misclassified as galaxies on both plates. A match between the APM catalog and the Véron-Cetty \& Véron 1998 quasar catalog found that $10 \%$ of the Véron quasars brighter than $\sim 17.8$ in $E$ were classified as galaxies on both POSS-I plates by the APM (Paper I). However, $\sim 40 \%$ of such objects are identified as Seyfert galaxies in the Véron catalog, so that the APM galaxy classification is likely to be correct. The FBQS sample is certainly not complete for Seyfert galaxies due to the restriction to point-like optical sources. This morphology criterion similarly makes the FBQS 


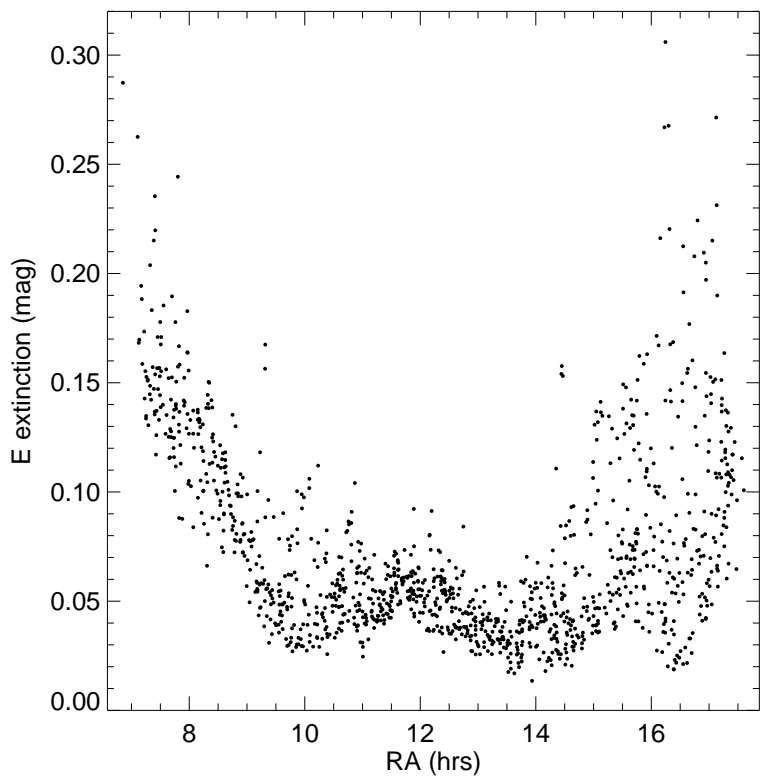

FIG. 3.- Extinction at $E$ as a function of Right Ascension for FBQS candidates. The $O$ extinction is 1.63 times larger. The extinction corrected magnitudes have been used for the color and magnitude cuts in defining the FBQS sample.

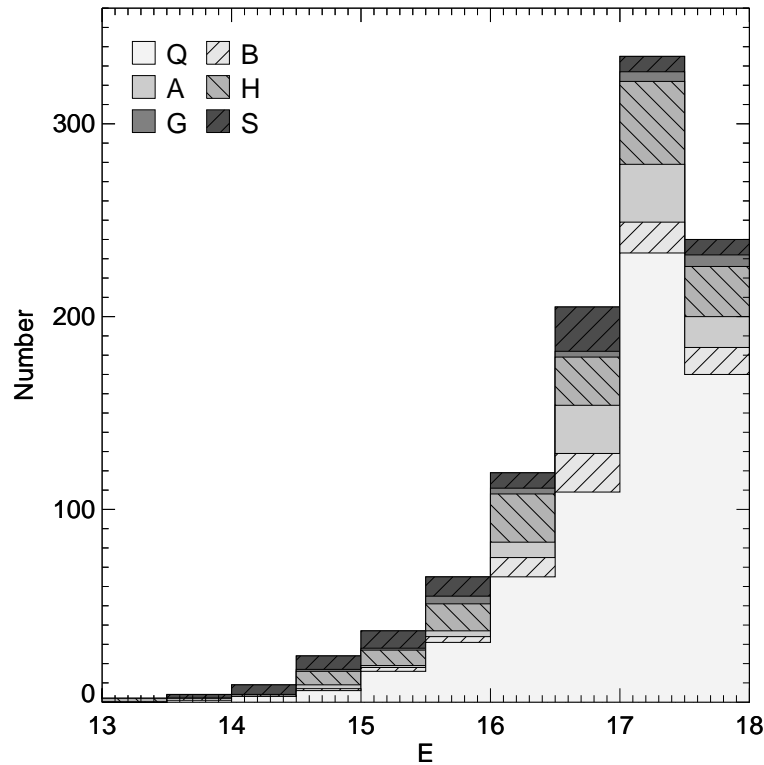

(a)

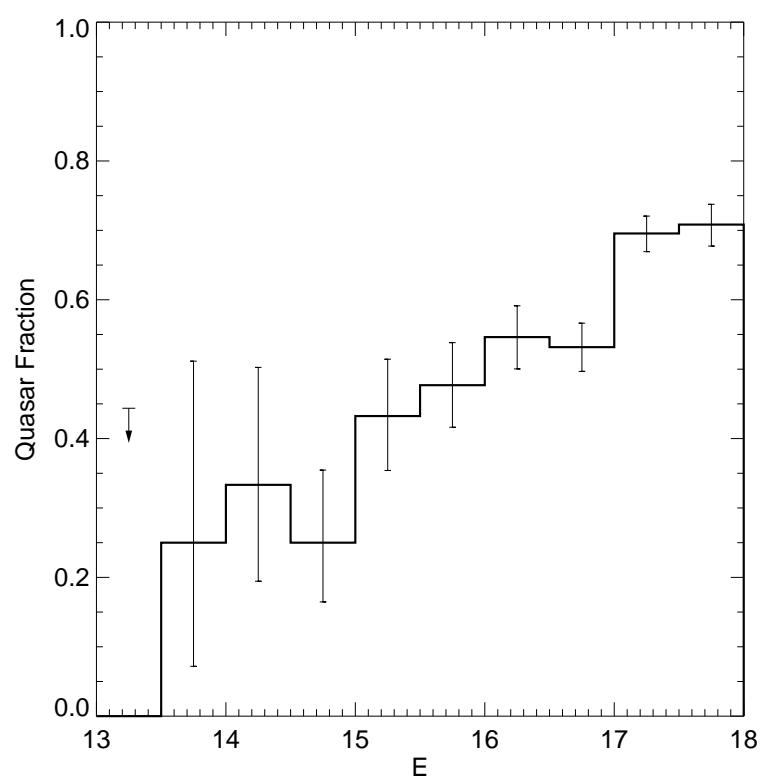

(b)

FIG. 4.- (a) Histogram of extinction-corrected $E$ (POSS-I red) magnitudes for FBQS candidates with identifications. Note that the 17.5-18.0 magnitude bin only includes objects between $17.5<E<17.8$. (b) Fraction of quasar identifications as function of $E$ magnitude. 
sample somewhat incomplete at low redshifts $(z \lesssim 0.2)$, where the quasar host galaxy may be bright enough to skew the APM classification.

Requiring a point-like optical morphology may also discriminate against another interesting class of quasar: gravitationally lensed (or binary) objects. Gravitational lenses with two roughly equally bright images separated by $\sim 2$ to $\sim 10$ arcseconds are likely to be recorded as a single non-stellar object in the APM catalog; they are also likely to have less accurate positions in both the radio and the optical due to their extent. Such wide lenses are probably best identified using higher-resolution images than can be obtained from photographic Schmidt surveys. However, most gravitationally lensed quasars have smaller separations than this and so will be included in our sample despite not being true point sources. In an imaging search for lenses among the FBQS sample (Schechter et al. 1998), we have so far found two lensed objects; both have component separations of $\sim 1$ arcsec or less and are unresolved by the APM, which classifies each as stellar on both plates. We have also identified one binary quasar (Brotherton et al. 1999) with a separation of 2.3 arcsec, which is similarly classified as stellar on both plates, although it is slightly too faint to be in the FBQS sample.

\subsection{Optical Color}

Lastly, we excluded FIRST sources with very red optical counterparts $(O-E>2)$ since in the pilot study no quasars were this red. At the risk of missing a few intrinsically red or high-redshift quasars, this cut substantially reduces the number of candidates to observe. A histogram of the colors of all the quasars identified to date (Fig. 5a) shows very few close to the color limit and a very low discovery efficiency near $O-E=2$ (Fig. 5b). There are only 7 confirmed quasars with dereddened $O-E \geq 1.8$. Although we cannot exclude a large population of red quasars, there are very few quasars near our color boundary, whereas the non-quasar contamination of the candidate list has a strong dependence on color. Figure 5(a) also indicates the color distribution of the BL Lacs, AGN, H II/star-forming galaxies, and normal galaxies identified in our survey.

Our color cut does discriminate against one known class of red quasars: very high redshift quasars $(z \gtrsim 3.5)$. Hook et al. 1995 describe the evolution of APM/POSS-I colors with redshift and show that when the Lyman forest absorption moves into the $O$ band, the $O-E$ color becomes dramatically redder. This trend is visible in a plot of $O-E$ versus $z$ for the FBQS quasars (Fig. 6), where the colors of $z>3$ quasars are distinctly redder than those at lower redshifts. A prime goal of extending the FBQS sample to include redder objects would be to find high redshift quasars (e.g., Hook, Becker, McMahon, \& White 1998), but in view of the contamination of the red objects in the APM catalog by galaxies, due mainly to the poor morphology discrimination feasible with the POSS-I plates, we decided it was best for the FBQS to postpone a red quasar search until we have higher quality optical images (e.g., from the Sloan Digital Sky Survey and other deeper, higher resolution CCD surveys.)

In any case, few if any high redshift quasars were missed as a result of our color cut. The limit at $O-E=2$ is substantially redder than the typical unreddened quasar color $(O-E \sim 0.5$, Fig. 5), so it does allow for significant absorption in the $O$ band; and we do not expect to find many $z>3.5$ quasars brighter than $E=17.8$ mag simply because of the large ultraviolet luminosities required. If there is a closer population of quasars that is heavily reddened by either intrinsic or intervening dust absorp- tion, the absorption at $E$ would have to be several magnitudes in order to produce a differential extinction of a magnitude or more in $O-E$. Consequently, such objects would also be too faint to be included in this bright sample.

\subsection{Radio Properties}

There are no cuts to the sample based on radio flux density or radio morphology (other than the unavoidable requirement that the radio source have at least a weak core component, as mentioned above.) However, the FIRST catalog itself introduces several minor selection effects (BWH95; WBHG97). The FIRST sensitivity limit is somewhat non-uniform on the sky, with small ( $\sim 15 \%$ peak-to-peak) variations due to the observing strategy and large variations due to decreased sensitivity in the vicinity of bright sources. The fraction of the survey area affected by sensitivity variations is small: $86 \%$ of the FBQS area has a $5 \sigma$ flux density limit of $\leq 1 \mathrm{mJy}$ (the catalog has a hard limit at $1 \mathrm{mJy}$ even when the images are slightly better than that), and $98 \%$ of the survey has a limit $<1.25 \mathrm{mJy}$. The FIRST coverage map (WBHG97; available through the FIRST web pages) gives the sensitivity as a function of position.

The FIRST survey detection limit applies to the peak flux density of sources rather than to the integrated flux density. Consequently, extended sources with total fluxes greater than 1 mJy may not appear in the catalog because their peaks fall below the detection threshold. For the objects selected in the FBQS, we expect the insensitivity of FIRST to extended sources to contribute less to the incompleteness than the requirement that FBQS quasars must have a nuclear radio component in order to meet the positional coincidence criterion. The latter, for example, makes the FBQS incomplete to the population of radio quasars that have extended, fossil radio lobes but no active nuclear emission (which must exist, though they are rare.)

The FBQS sample constitutes a significantly different population of quasars than has been seen in previous radio surveys. Figure 7 shows the distribution of radio flux densities for the confirmed quasars. The number of quasars increases dramatically with decreasing radio flux density near the FIRST survey limit. This is because at $1 \mathrm{mJy}$, the FIRST survey is probing the larger, radio-quiet (but obviously not radio-silent) quasar population. This is also clearly seen in Figure 8(a), which plots the monochromatic radio luminosity versus redshift for FBQS quasars. FIRST is sensitive to a large population of low radioluminosity quasars, especially at low redshift.

In $\$ 2.2$ we discussed the importance of the Malmquist bias vis a vis the E magnitude cutoff for the sample. The rapid increase in the FBQS counts toward our 1 mJy flux density cutoff implies that there is also a radio Malmquist bias in our sample. The slight nonuniformity of the FIRST survey flux density limit directly affects the FBQS sample and could create the appearance of large scale structure, since regions of higher sensitivity will have an excess of quasars. This effect must be accounted for in analyses of large-scale structure in the FBQS sample.

Even though the number of quasars increases sharply below a flux density of several mJy, the number of non-quasar candidates rises even faster such that the efficiency of identifying quasars decreases as the radio flux density decreases (see Fig. 7b). Nonetheless, quasars near the FIRST flux density limit are worth the effort because it is here that the FBQS is making a unique contribution, probing the transition between radio-loud 


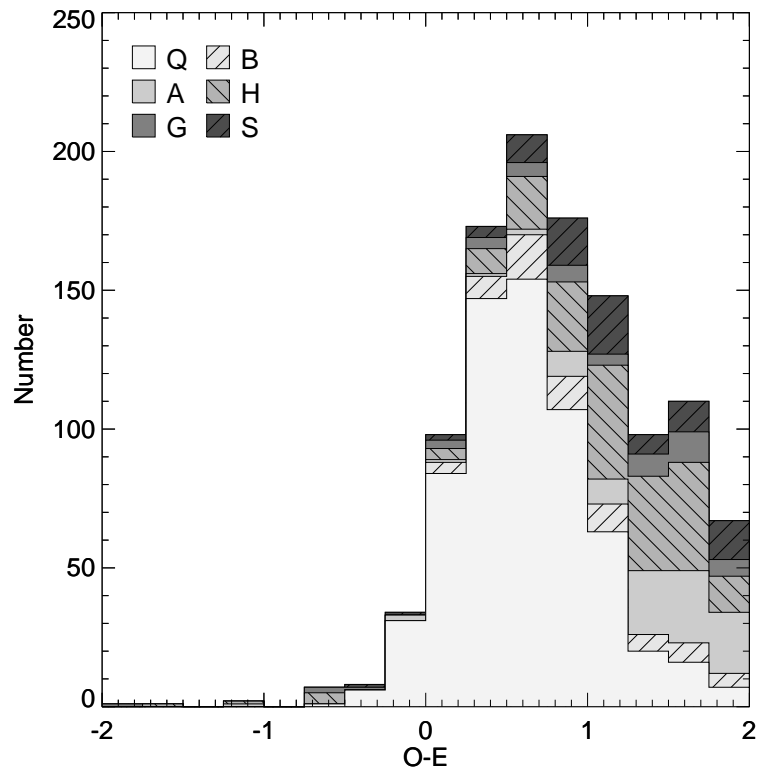

(a)

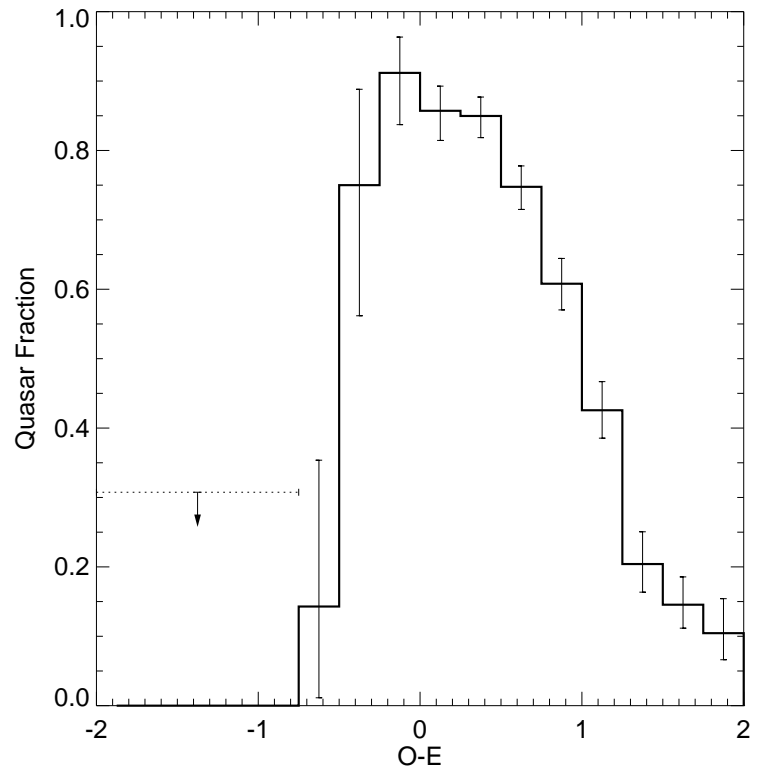

(b)

FIG. 5.- (a) Histogram of extinction-corrected colors of identified FBQS candidates. (b) Fraction of quasar identifications as function of color.

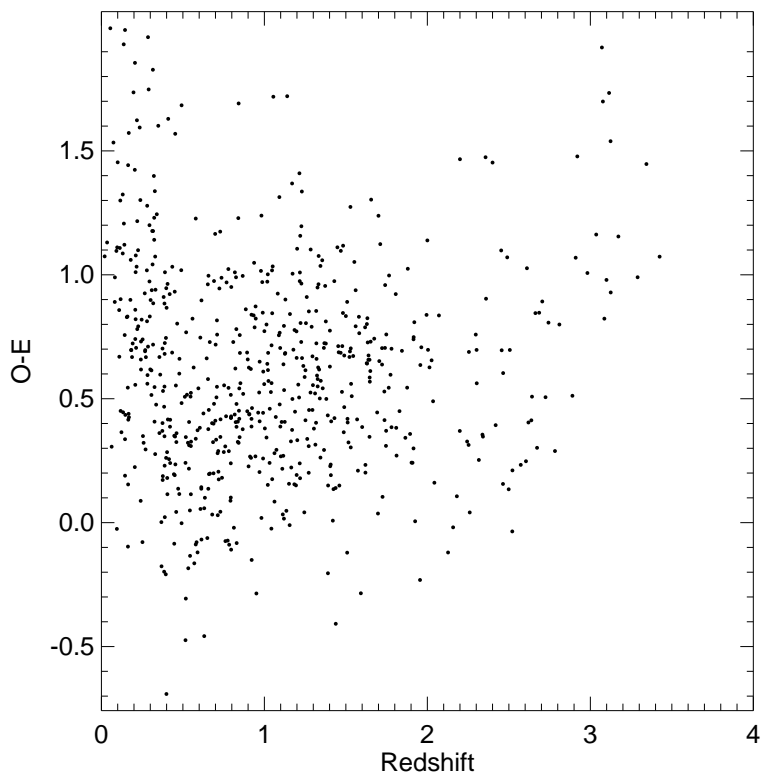

FIG. 6.- Optical color versus redshift for FBQS quasars. At $z>3$ the colors of the quasars begin to redden, so that high- $z$ quasars may be lost from the FBQS sample due to the color cut at $O-E \leq 2$ (Hook et al. 1995). 


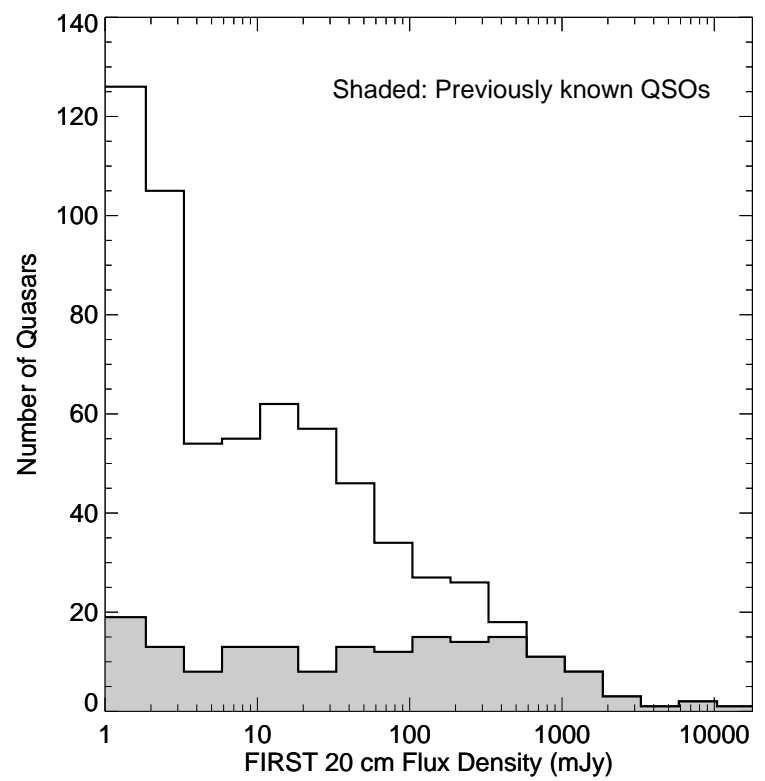

(a)

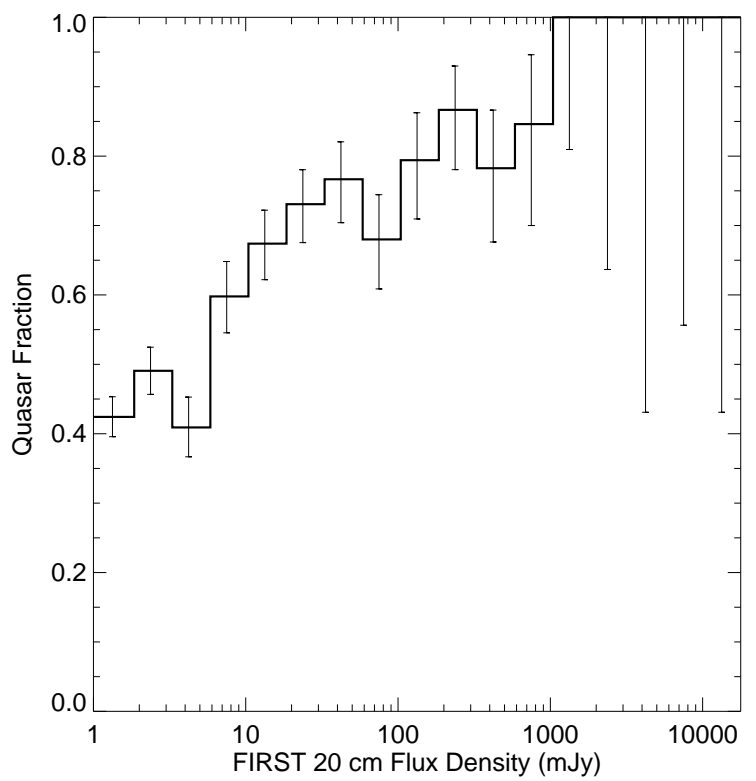

(b)

FIG. 7.- (a) Histogram of FIRST integrated radio flux densities of FBQS quasars. Shaded: previously known quasars. (b) Fraction of FBQS candidates identified as quasars as function of radio flux density. The flux densities come from the FIRST catalog and so include only the core radio emission.

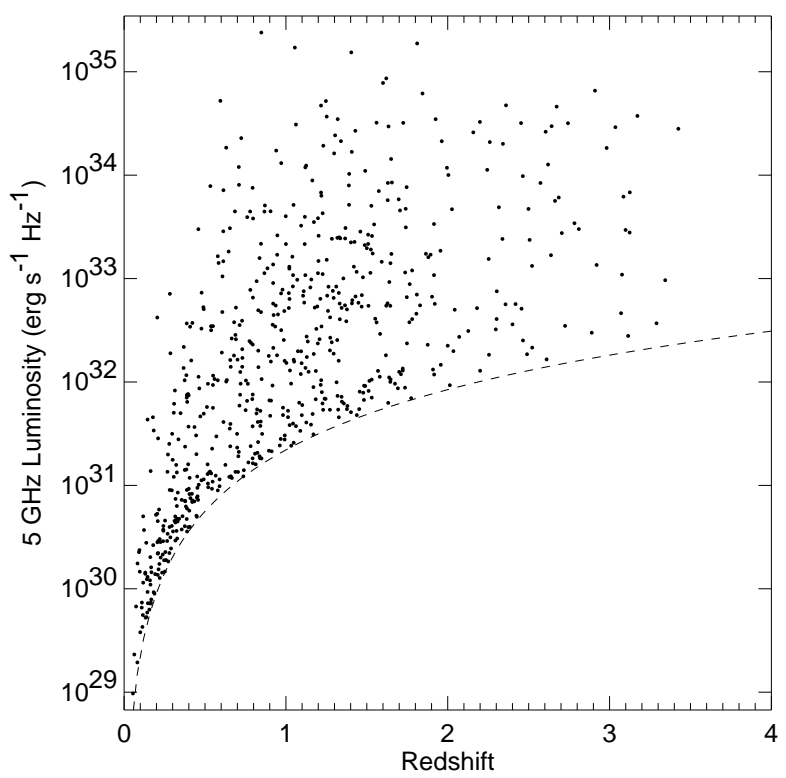

(a)

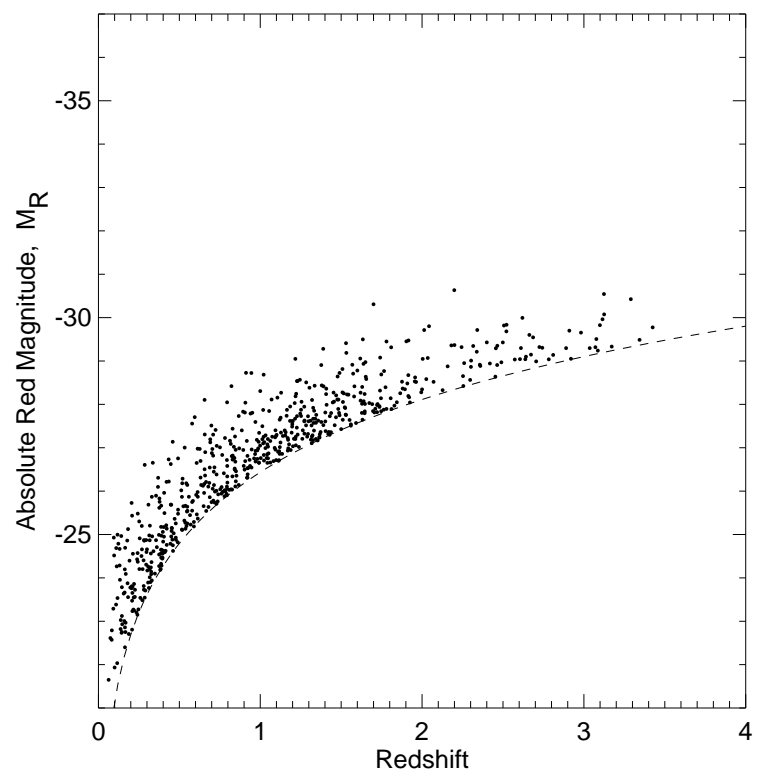

(b)

FIG. 8. - FBQS quasar luminosities versus redshift in the radio and optical. (a) Radio luminosity at a rest frequency of 5 GHz versus redshift, using spectral index $\alpha=-0.5$. (b) Absolute red magnitude versus redshift, using spectral index $\alpha_{o p t}=-1$ for the $K$-correction. Dashed lines show $1 \mathrm{mJy}$ FIRST detection limit and $E=17.8$ APM magnitude limit. The radio luminosities have a much larger dynamic range and do not crowd as closely against the detection limit as do the optical magnitudes. 
and radio-quiet objects. The distribution of objects in the transition region is discussed further below ( $\$ 5.4)$.

\subsection{How Efficiently Can Quasars Be Selected?}

The fraction of FBQS candidates that turns out to be quasars is, as shown above, a function of magnitude, color, radio-optical separation, etc. This raises an interesting question: how efficiently can we select quasars, using only the information that is available before taking spectra? In other words, how well can we maximize the number of quasars discovered per spectrum taken?

It is clear that by setting tight limits on the separations (Fig. 2) and colors (Fig. 5), and by observing only the optically fainter (Fig. 4) and radio-brighter (Fig. 7) objects, we could eliminate many of the FBQS candidates that turn out not to be quasars and so increase our efficiency. However, the completeness of the sample would suffer greatly using such a strategy, and many of the quasar candidates rejected would be among the more interesting types (e.g., radio-quiet, radio-intermediate, and high-redshift objects.)

To explore this question quantitatively, we have applied artificial-intelligence methods to classify the FBQS candidates according to their a priori (before taking spectra) probability of being quasars, $P(Q)$. There are a number of classification methods that we could have used (neural nets, nearest-neighbor algorithms, clustering methods, Bayesian classifiers, etc.); we chose to use the oblique decision tree classifier OC $1^{14}$ (Murthy, Kasif, \& Salzberg 1994), which we have found to represent a good compromise between the competing goals of being fast in training and in application and of generating accurate, understandable classification algorithms. In the past this method has been successfully applied to cosmic ray identification in HST images (Salzberg et al. 1995), to star-galaxy discrimination for the Guide Star Catalog-II (White 1997), and to the problem of flagging sidelobes appearing in the FIRST catalog (WBHG97).

\subsubsection{Description of $O C 1$}

OC1 takes as input a training set of objects that have known classifications; in our case, we use all objects with spectra as the training set. Each object is described by a vector of numerical features (magnitudes, colors, and so on.) OC1 constructs a decision tree that accurately classifies the training set.

At each node in the tree a linear combination of the feature values plus a constant is computed; if the sum is positive, the right branch of the tree is taken, otherwise the left branch is taken. After each branch, there may be another decision test node, or the tree may terminate (a "leaf" node). To classify an unknown object, one starts at the tree's root node and works down the decision tree, doing a series of tests and branches, until a leaf node is reached. The OC1 program attempts to produce trees that have pure samples of training-set objects at each leaf node (i.e., in the current case it tries to separate the training objects so that each leaf has nearly all quasars or nearly all non-quasars.) The unknown object is classified as quasar or not depending on which is the majority type of object at the final leaf.

\subsubsection{Voting Decision Trees}

We have improved on the accuracy of the classification by using not just a single tree, but rather a group of 10 trees that vote. This multiple-tree approach has been shown to be quite effective (Heath, Kasif, \& Salzberg 1996). Searching for a good decision tree is an extremely difficult optimization problem; to find an approximate solution, $\mathrm{OC} 1$ uses a complex search algorithm that includes some randomization to avoid the classic problem of getting stuck in local minima in the manydimensional search space. Thus, one can run OC1 many times using different seeds for the random number generator to produce many different trees.

Heath, Kasif, \& Salzberg 1996 used a simple majority voting scheme: classify the object with each tree and then count the number of votes for each class. We have improved on this by using a weighted voting scheme, where each tree splits its vote between the quasar and non-quasar classes depending on the populations of the two types from the training set at that leaf. If an object winds up at a leaf node with $N$ training set objects of which $Q$ are quasars, the tree's single vote is split into $(Q+1) /(N+2)$ in favor and $(N-Q+1) /(N+2)$ against a quasar classification. (The particular form used was derived from the binomial statistics at the leaf.) We considered a variety of other voting schemes, but this one appeared to be the most accurate and robust.

\subsubsection{Testing the Classification Accuracy}

The classifier was tested using 5-fold cross-validation. The training set (which consists of $\sim 1000$ objects with spectra) is divided into 5 equal-sized, randomly selected subsets (or folds). Setting aside the first fold, 10 decision trees are constructed by training on the other 4 folds. Then the trees are tested on the first fold, which was not used in the training, computing $P(Q)$ for each object in the fold. This process is repeated 5 times, each time holding back a different fold; when complete, we have used each object for testing exactly once. This technique, which is a standard approach, avoids the over-optimistic results for classification accuracy one would get if one simply trained on all the data and then tested the classifier on the same data.

\subsubsection{Results}

The approach may sound complicated, but the ultimate result is simple - for each object, we compute the probability $P(Q)$ that the object is a quasar. A perfect classifier would assign all quasars $P(Q)=1$ and all non-quasars $P(Q)=0$; in a non-ideal case, we want to see quasars with $P(Q)$ values concentrated near unity and non-quasars near zero.

Figures 9(a) and 9(b) show the distribution of $P(Q)$, the probability that an object is classified as a quasar, using 10 voting trees. The distinction between the two cases is the set of features used for classification. Example (a) used 7 parameters: $E, O-E, \log S_{p}$ (where $S_{p}$ is the FIRST peak flux density), $S_{i} / S_{p}$ (where $S_{i}$ is the FIRST integrated flux density), the radiooptical separation, and the two PSF parameters from the APM catalog that measure how point-like the object is on the $O$ and $E$ plates. Example (b) omitted the parameter $O-E$, so that no color information was included.

Both panels of Figure 9 clearly show that the classifier does a good job of separating the FBQS candidates into high and low probability quasars. In both cases the distribution is encouragingly close to that of an ideal classifier. The quasar/non-quasar separation is somewhat better when the $O-E$ color information is used: the distribution is more strongly peaked at the low and high $P(Q)$ ends. We created the color-free classifier because an examination of the

\footnotetext{
${ }^{14} \mathrm{OC} 1$ is available via anonymous ftp from http://www. cs.jhu. edu/salzberg/announce-oc1.html
} 


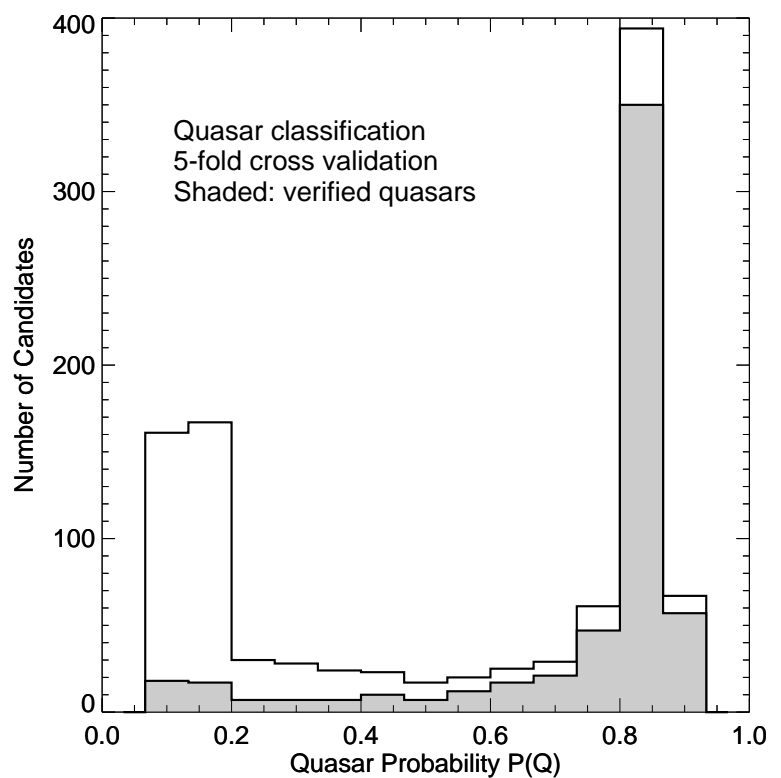

(a)

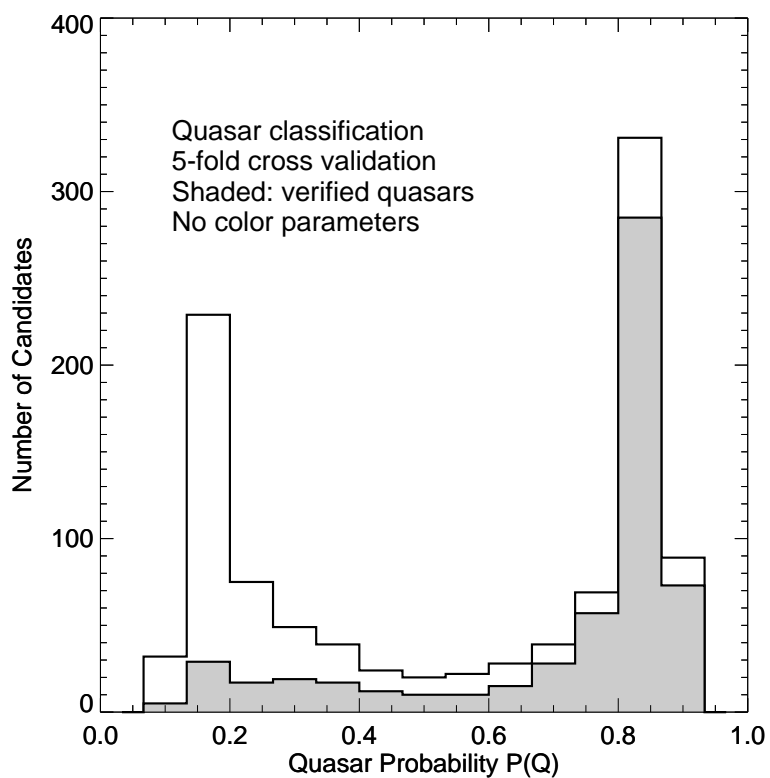

(b)

FIG. 9.- Distribution of the a priori probability that an object is a quasar, $P(Q)$, for all identified FBQS candidates. The shaded histogram is the distribution of confirmed quasars, while the unshaded area represents non-quasars. A perfect classifier would put all the quasars at $P(Q)=1$ and all the non-quasars at $P(Q)=0$. The probabilities were calculated with decision trees using 5-fold cross-validation, as described in the text. (a) $P(Q)$ computed using all available parameters, including $O-E$ color. (b) $P(Q)$ computed without color. The second approach is less efficient at distinguishing quasars from non-quasars, but it does not discriminate against high-redshift or heavily reddened broad absorption line quasars as does the method including color. In both cases, the great majority of the $P(Q)<0.2$ quasars are at low redshift $(z<0.3)$ and are presumably slightly resolved.

quasars with $P(Q)<0.2$ (the shaded bins below 0.2 in Fig. 9a) shows that 4 of the 36 misclassified objects are in the interesting category of red quasars, either because they are high redshift $(\mathrm{J} 092104.4+302031, z=3.34)$ or because they have strong broad absorption lines (BALs; J105427.1+253600, $\mathrm{J} 120051.5+350831$, and $\mathrm{J} 132422.5+245222$ ). If we exclude color as a classification criterion, the number of $P(Q)<0.2$ quasars stays about the same ( 34 objects), but the misclassified candidates are neither high-redshift nor BAL quasars.

In both cases, the great majority of the low $P(Q)$ quasars are low redshift objects: $24 / 34(70 \%)$ of the $P(Q)<0.2$ objects have $z<0.25$. These objects are probably being recognized as slightly resolved on the POSS-I plates and so are, in some sense, rightly classified as non-quasars (since they are perceptibly non-stellar.)

At the other extreme, the great majority of the high $P(Q)$ candidates that are not quasars turn out to be BL Lacs. Using the classifier with color, there are 419 objects with $P(Q)>0.85$, of which $373(89 \%)$ are quasars and $46(11 \%)$ are non-quasars. But 31 of the 46 non-quasars are BL Lacs. If we combine the BL Lacs with the quasars, an astounding $96.4 \%$ of the high probability objects are quasars or BL Lacs and only $3.6 \%$ are other types of objects.

Figure 10 shows the fraction of candidates that turn out to be quasars as a function of $P(Q)$. The distribution follows well the expected linear form assuming that $P(Q)$ is, in fact, a good estimate of the probability that an object is a quasar.

Finally, Figure 11 shows how the completeness $C$ and reliability $R$ of the sample vary as we change the $P(Q)$ threshold, $P_{C}$, above which candidates are accepted into the sample. If we set $P_{C}=0$, all objects in the original FBQS sample are included, and the resulting sample is $100 \%$ complete (at least, it includes all the objects in the current paper) but is only $55 \%$ reliable (so
$45 \%$ of the candidates turn out to be non-quasars.) As $P_{C}$ increases, the reliability of the sample increases dramatically at little cost in completeness. If color is used by the classifier, one can construct a sample that is about $80 \%$ reliable while still being $90 \%$ complete, or one can choose higher reliability (up to $89 \%)$ if lower completeness (70-80\%) is acceptable. The results when color is not used are not quite as good, but still it is possible to construct an impressively pure sample at modest cost in completeness.

The way we might use this information depends on the scientific goals of the quasar survey. For the FBQS, our scientific interest is in taking a census of quasars to understand their demographics and physics. Consequently, we put a high priority on completeness and are willing to pay for it by spending extra telescope time to get spectra of lower probability candidates. We are loathe to risk losing high redshift and heavily absorbed BAL quasars from our sample, so we prefer to restrict the use of color and to aim instead for a highly complete sample.

On the other hand, if one is interested in quasars mainly as sources to probe the intergalactic medium, it could be appropriate to sacrifice completeness and the occasional red quasar in order to get a highly pure sample. In a search for quasars at particular locations, for example near other quasars or behind galaxy clusters, this approach can be used to identify excellent candidates with high probability, before a single night of telescope time has been used on spectroscopy.

To put our money where our mouths are, Table 8 gives our estimate (using color parameters) of the probability $P(Q)$ that each object is a quasar for the FBQS candidates that have not as yet been identified. As these objects are observed, we are confident that our predictions will be (in the mean) borne out. While we expect to use our simple sample-definition criteria as we continue expand the FBQS sample, we are already using 


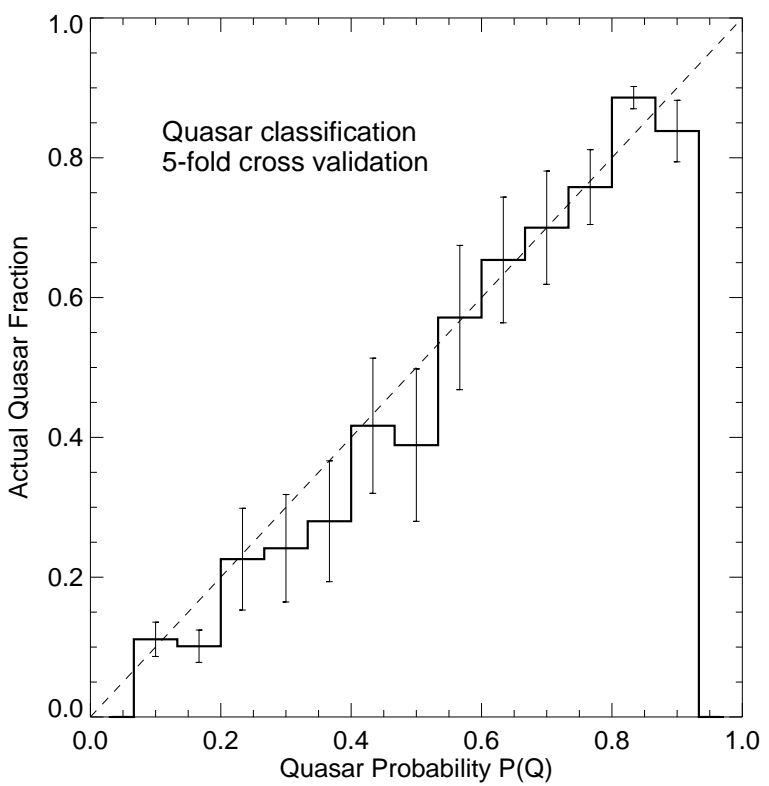

FIG. 10.- Fraction of candidates that are confirmed as quasars as a function of $P(Q)$. This was computed including color as one of the parameters, but the results without color are essentially identical. The dotted line has a slope of unity. The probability is a good predictor of the likelihood that a candidate will turn out to be a quasar.

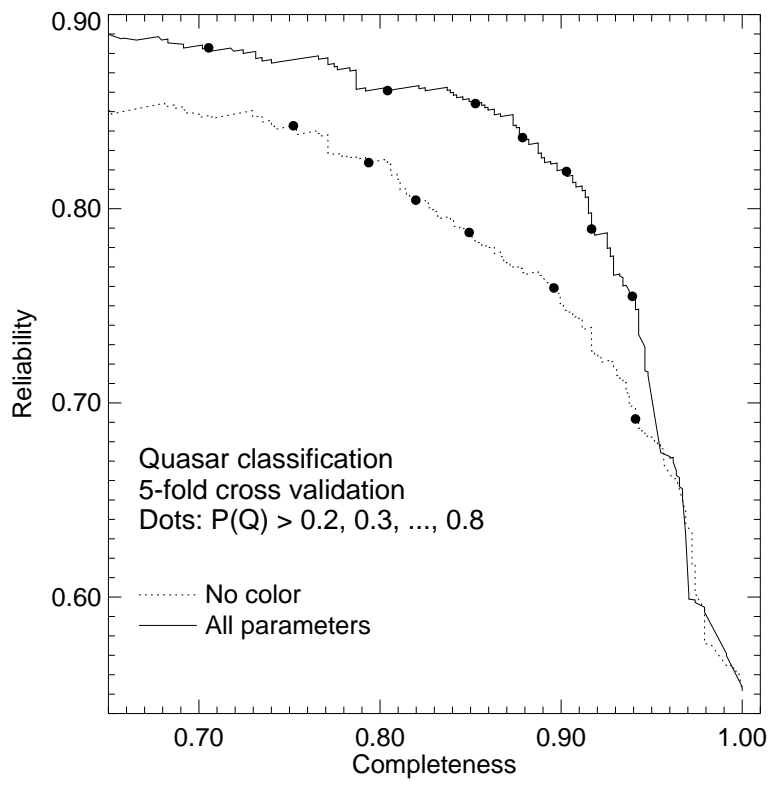

FIG. 11.- Completeness $C$ and reliability $R$ of the sample as a function of the threshold selected for the probability $P(Q)$. The completeness is the fraction of quasars from the complete FBQS sample that are included at the selected $P(Q)$ threshold; the reliability is the fraction of selected candidates that are quasars. The solid line is computed using all parameters; the dashed line excludes color. The FBQS sample presented in this paper is the point at $C=1, R=0.55$ (i.e., the sample is complete but only $55 \%$ of the candidates turn out to be quasars.) The efficiency of the quasar search (determined by the reliability of the prediction) can be increased to 89\% using color (85\% without color), if completeness is not crucial. Even with completeness greater than $90 \%$, the reliability can be higher than $80 \%$. 
the $P(Q)$ probabilities to assist in prioritizing targets for observation. Further improvements are probably possible utilizing advance knowledge of the X-ray and infrared properties of the candidates.

\subsection{Summary of Incompleteness of the FBQS Sample}

In brief, the selection criteria applied to the FBQS sample lead to several possible kinds of incompleteness and bias:

- The requirement of close positional coincidence excludes extended, lobe-dominated radio sources with no core component.

- The $E$ magnitude cut combined with the steep quasar luminosity function leads to unavoidable Malmquist bias in the sample. There is also a radio Malmquist bias from the FIRST flux density limit, which varies slightly as a function of position on the sky.

- The requirement that the objects be stellar on the POSSI plates leads to incompleteness for low-redshift objects (which may appear resolved) and will bias against the discovery of wide gravitational lenses. Since the APM classifier is not perfect, there can also be truly stellar objects that are misclassified as galaxies on both plates and so are missing from our sample.

- The red color cut makes the sample incomplete for very high redshift $(z \gtrsim 3.5)$ and heavily obscured quasars; however, most such objects are likely to be optically too faint to be in the sample anyway.

- The FIRST catalog itself is incomplete for weak extended sources.

It is important to note that this survey is targeting quasars and hence the selection criteria have been optimized for quasars. Generally speaking, what works for quasars does not necessarily work for BL Lac objects, AGNs, or H II galaxies. In particular, requiring the optical morphology to be stellar and the optical colors to be bluer than $O-E=2$ excludes very few quasars, but probably excludes the majority of the other types of objects. So even though we are finding examples of such objects, the lists should not be taken as complete.

For most purposes, however, these selection effects do not seriously affect the utility of the sample for statistical studies of quasars. The biases in optically selected samples are generally worse in that they are more difficult to characterize and correct. For example, the differential $K$-correction for broad-absorption line quasars introduces a complex selection in number counts versus redshift. The FBQS at least partly redresses these biases (e.g., by allowing much redder objects to be included.) Indeed, the FBQS is, within its limits, perhaps the most complete largearea quasar survey that exists.

\section{OPTICAL SPECTROSCOPY}

Spectra for the quasar candidates are being collected at five different observatories: the 3-m Shane telescope at Lick Observatory, the 2.1-m telescope at Kitt Peak National Observatory ${ }^{15}$, the 3.5-m telescope at Apache Point Observatory ${ }^{16}$, the $6 \times 1.8$ $\mathrm{m}$ Multiple Mirror Telescope ("MMT classic"), and the 10-m Keck-II telescope. The spectrographs at the five observatories all have different resolutions and wavelength coverage, which are summarized in Table 1 . The observations are made in a wide variety of atmospheric conditions ranging from photometric to cloudy with both good and bad seeing. Many of the candidates have been observed more than once. We have observed almost all the candidates in the original candidate list, even those with previous identifications. (In several cases we have found errors in published redshifts or positions. Objects found to be discrepant compared with the Véron-Cetty \& Véron 1998 catalog are noted in the table.)

\section{SPECTROSCOPIC RESULTS}

Applying all the criteria described in $\$ 2$ results in a list of 1238 candidates in 2682 square degrees, of which 169 are previously known quasars. In Tables 2-7 we present a list of all the candidates in the FBQS sample with spectral classifications. For each object we list the FIRST catalog RA and $\operatorname{Dec}^{17}$ (J2000), the recalibrated and extinction-corrected $E$ and $O$ magnitudes, the red extinction correction $A(E)$, and the FIRST peak and integrated radio flux densities. The radio-optical positional separation and APM star-galaxy classification (used to define the sample) are also given. The objects have been segregated into 6 tables by their optical spectral classification. Quasars are listed in Table 2, narrow line AGN in Table 3, BL Lac objects in Table 4, H II/star-forming galaxies in Table 5, galaxies without any emission lines in Table 6, and stars in Table 7. The criteria used to classify spectra are given below. Table 8 lists the objects for which spectra have not yet been obtained.

For the purposes of the FBQS, we have classified any object with broad emission lines as a quasar: i.e., we do not make a distinction between quasars and Seyfert 1 galaxies. We also make no distinction between quasars and broad-line radio galaxies, the radio-loud counterparts to Seyfert 1 galaxies. The absolute blue magnitude is given for objects with redshifts, so a conventional cut at $M_{B}=-23$ can be made if desired to exclude lower-luminosity objects. Of the 636 quasars, 50 fall into this lower luminosity category. (The APM magnitudes are far too bright for extended objects with apparent magnitudes brighter than $\sim 12 \mathrm{mag}$, so the high luminosities implied by the $M_{B}$ values for such objects should not be taken seriously. We have appended a note in the table for such objects giving the blue magnitude from $\mathrm{NED}^{18}$ when available.)

BL Lac classification is based on the traditional definition presented in Stocke et al. 1991: all objects with emission line rest-frame equivalent widths less than $5 \mathrm{~A}$ and/or $4000 \mathrm{~A}$ break contrasts $\left(\mathrm{Br}_{4000}\right)$ less than $25 \%$ appear in Table 4 . When emission lines or $\mathrm{Br}_{4000}$ have been measured, we list the quantities in the notes. Although this definition has been shown to be

\footnotetext{
${ }^{15}$ Kitt Peak National Observatory, NOAO, is operated by the Association of Universities for Research in Astronomy, Inc. (AURA), under cooperative agreement with the National Science Foundation

${ }^{16}$ The Apache Point Observatory is owned and operated by the Astrophysical Research Consortium.

${ }^{17}$ The preferred naming convention for objects in the sample is "FBQS Jhhmmss.s+ddmmss", where the Right Ascension and Declination given in the table are truncated (not rounded.) This is consistent with the IAU-approved naming convention for FIRST radio sources.

${ }^{18}$ The NASA/IPAC Extragalactic Database (NED) is operated by the Jet Propulsion Laboratory, California Institute of Technology, under contract with the National Aeronautics and Space Administration.
} 
TABLE 1

CHARACTERISTICS OF SPECTROGRAPHS

\begin{tabular}{lcc}
\hline \hline \multicolumn{1}{c}{ Telescope } & $\begin{array}{c}\text { Approximate } \\
\text { Wavelength Range (A) }\end{array}$ & Resolution (A) \\
\hline Lick 3-m & $3600-8150$ & 6 \\
KPNO 2.1-m & $3700-7400$ & 4 \\
APO 3.5-m & $3650-10000$ & 10 \\
MMT 6 $\times 1.8-\mathrm{m}$ & $3600-8500$ & 8 \\
Keck-II 10-m & $3800-8800$ & 8 \\
\hline
\end{tabular}

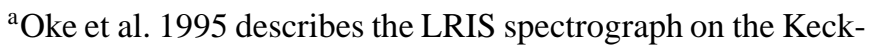
II telescope.

too restrictive, excluding some objects that otherwise exhibit BL Lac-like properties (see Marchã et al. 1996 and LaurentMuehleisen et al. 1998), our preliminary analysis indicates that broader criteria admit many objects into the BL Lac class that do not properly belong there. This is mainly because the FBQS candidate objects are more than two orders of magnitude fainter in the radio than the Marchã et al. sample used to define the newer BL Lac criteria. The FBQS selection thus admits many objects with very low radio luminosities that exhibit weak optical emission and absorption features like the high radio luminosity objects in Marchã et al. Many of these objects are clearly not BL Lacs despite being consistent with the broader optical selection criteria. A full analysis of the BL Lac content of the FBQS sample is underway (Laurent-Muehleisen et al. 1999) and to avoid including non-BL Lacs in the table, we list only those objects that adhere to the strictest criteria.

We divide objects with narrow emission lines into narrowline AGN and $\mathrm{H}$ II galaxies based on the ratios of [N II] to $\mathrm{H} \alpha$ and [O III] to $\mathrm{H} \beta$. An object is classified as a narrow-line AGN if [N II] is more than $60 \%$ of $\mathrm{H} \alpha$, or (when $\mathrm{H} \alpha$ is not observed) if [O III] is more than twice $\mathrm{H} \beta$; otherwise it is designated as an H II galaxy (Osterbrock 1989). The classification based on $[\mathrm{O} \mathrm{III}] / \mathrm{H} \beta$ can be ambiguous and so the H II/AGN separation is less reliable for higher redshift objects.

Some of the objects classified as H II galaxies are likely to be narrow-line Seyfert 1 galaxies, which can have similar line ratios (Osterbrock \& Pogge 1985). For this paper we have not attempted to separate the two types of objects.

All other objects are classified as either normal galaxies (with no obvious emission lines) or stars. Galaxies flagged as previously known in Table 6 have been identified in NED; in most cases we have not obtained spectra, so their emission line (and $\mathrm{Br}_{4000}$ ) status should be considered less certain than the other objects in the table.

Note that most stars in the sample are likely to be chance coincidences with the radio sources. The APM positions for stars are degraded by 40 years of proper motion between the POSSI and FIRST epochs; coupled with the rarity of radio emission from stars, this prevents a useful match between FIRST and APM for stars. Recent epoch stellar catalogs (ideally with proper motions) are required to study the radio emission from stars using FIRST (Helfand et al. 1999a). One object listed in the stars table, J163302.6+234928, is detected in the ROSAT
All Sky Survey Bright Source Catalog (Voges et al. 1996) and is therefore likely to be a true stellar radio source identification. Another, J164018.1+384220, is actually a known halopopulation planetary nebula (Barker \& Cudworth 1984). It is included with the stars because it is a Galactic object (and it was formerly a star.) A dozen or more of the other objects in Table 7 are also likely to be real radio stars, but current epoch optical positions will be needed to secure the identifications.

In Tables 2-6, we list the measured redshift where available (redshifts are unknown for many of the BL Lac objects). Redshifts for quasars and BL Lac objects were computed by crosscorrelating the spectra with templates; for the quasars, a template constructed from the FBQS objects themselves was used (Brotherton, Tran, et al. 1999). We use the redshift to calculate for each object the radio luminosity $L_{R}$ at a rest frequency of $5 \mathrm{GHz}$ (assuming a radio spectral index of $\alpha=-0.5$ ), the absolute $B$ magnitude $M_{B}$, and, as a measure of radio loudness, the ratio $R^{*}$ of the $5 \mathrm{GHz}$ radio flux density to the 2500 A optical flux in the quasar rest frame (assuming $\alpha_{o p t}=-1$ and using the definition of Stocke et al. 1992). We use the (APS-calibrated) APM $O$ magnitude as a direct estimate of $B$, and we do not correct the optical magnitude for the emission line contribution. The cosmological parameters $H_{0}=50 \mathrm{~km} \mathrm{~s}^{-1} \mathrm{Mpc}^{-1}, \Omega=1$, and $\Lambda=0$ were used in the luminosity calculations. When the redshift is not available we omit $M_{B}$ and $L_{R}$, and we compute $R^{*}$ from the ratio of the $5 \mathrm{GHz}$ flux density to the $2500 \mathrm{~A}$ flux as above, but no $K$-corrections are applied. There is also a comments column, which notes details of particular interest such as the presence of broad absorption lines or damped Lyman $\alpha$ absorption lines, whether an object was previously known in the Véron quasar catalog (Véron-Cetty \& Véron 1998), the Hamburg catalog (Hagen, Engels \& Reimers 1999) or in NED, association with a ROSAT X-ray source or an IRAS infrared source, etc.

The spectra for all the objects identified as quasars are displayed in Figure 16 (See related series of gif files on the astroph website).

\section{DISCUSSION}

Detailed analysis of the FBQS sample will be deferred to other papers, but we briefly discuss here both general properties of the sample and some interesting source classes. 


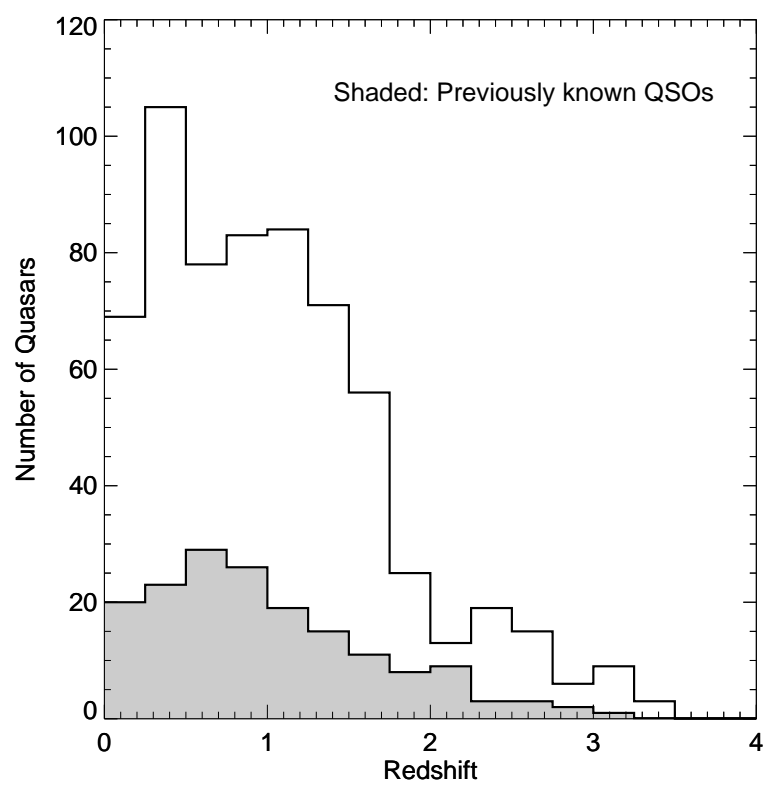

FIG. 12.— Histogram of redshifts for FBQS quasars. Shaded: previously known quasars.

\subsection{Redshift Distribution}

While the FIRST survey is clearly sensitive enough to detect quasars out to redshifts greater than 3 , the preponderance of FBQS quasars are at redshifts below 2 (Fig. 12). The precipitous drop beyond $z=2$ is attributable more to the limit in $E$ than to the limit in radio flux density. It is apparent in the redshift-radio luminosity distribution (Fig. 8a) that the highredshift quasars do not crowd against the radio detection limit; they are missing from the sample mainly because they are too faint in the optical (Fig. 8b). On the other hand, the lack of quasars at $z>3.5$ could be partly due to the red color cut used to define the sample, though the number of quasars beyond $z=3$ that are lost due to the color limit is certainly small $(\$ 2.4)$.

\subsection{Efficiency of the $F B Q S$}

The absolute efficiency of the FBQS in identifying quasars can be estimated by comparing the number of quasars found in the FBQS to the number expected from previous surveys. Figure 13 compares the sky density of FBQS quasars to estimates of the sky density of optically selected quasars as given by La Franca \& Cristiani 1997. A correction factor of 1.17 has been applied to the FBQS counts in the $B=17.4-17.9$ bin to account for objects that do not appear in the FBQS either because they are too faint on the red plate $(E>17.8)$ or because they are not yet identified (Table 8). The correction factor for the other bins is negligible, changing the raw counts by less than $3 \%$.

For quasars brighter than $B=16.4$ mag, the FBQS quasar density is indistinguishable from the density of optically selected quasars, so the efficiency is very high. The efficiency drops at fainter magnitudes, falling to $\sim 25 \%$ when $B=17.4-$ $17.9 \mathrm{mag}$. This does not mean that FIRST detects all optically bright quasars - we know that there are radio-silent quasars that would not be detected by FIRST. We can conclude, however, that FIRST detects the bulk of bright radio-quiet quasars and that the incompleteness of the FBQS (due mainly to very low radio fluxes) is similar to the incompleteness of bright optically selected samples (which is typically due to objects missed due to unusual color and emission line properties.)

An analysis of both quasars in the Véron-Cetty \& Véron 1998 and Hamburg (Hagen, Engels \& Reimers 1999) catalogs indicates that approximately $25 \%$ of all quasars brighter than $E=17.8$ are detected by the FIRST survey. The detected fraction is a bit lower for the Hamburg catalog - FIRST detects 17 out of $78(22 \%)$ quasars in the FBQS area. That may just be a reflection of the statistics of the small numbers involved, or it may indicate that the Hamburg survey is more efficient than previous optical surveys at detecting quasars with unusual optical properties.

\subsection{Advantages Over Optical Surveys}

When compared to previous quasar surveys, we find that the FBQS has two primary advantages. First, because it is a radio-selected survey, it has high selection efficiency, mainly because detectable radio emission from stars is extremely rare. Only about one in $10^{4} 12$ th magnitude stars has radio emission detectable by the FIRST survey (Helfand et al. 1999a). In contrast, X-ray selected samples are contaminated by ubiquitous stellar X-ray emission. The FBQS can cover a significantly larger sky area than optical objective prism surveys, with very much less telescope time required than for optical colorselected surveys. Perhaps the color-selection method will become comparably efficient with the advent of the wide-area, 5color Sloan Digital Sky Survey, but until then radio selection is the most efficient technique for discovering new quasars (once someone has done the hard work of making a deep, wide-area radio catalog.)

Second, the radio-selected FBQS can discover classes of quasars that are rare or unknown in other surveys because of biases against them. The best example in the FBQS sample are the extreme broad absorption line quasars that show low ionization iron absorption. Becker et al. 1997 reported the discovery of two such objects, J084044.4+363328 $(z=1.22$; included in this paper) and $\mathrm{J} 155633.8+351758(z=1.48$; too faint for the FBQS.) This paper includes 3 additional objects: $\mathrm{J} 104459.5+365605(z=0.701), \mathrm{J} 121442.3+280329(z=0.700)$, 


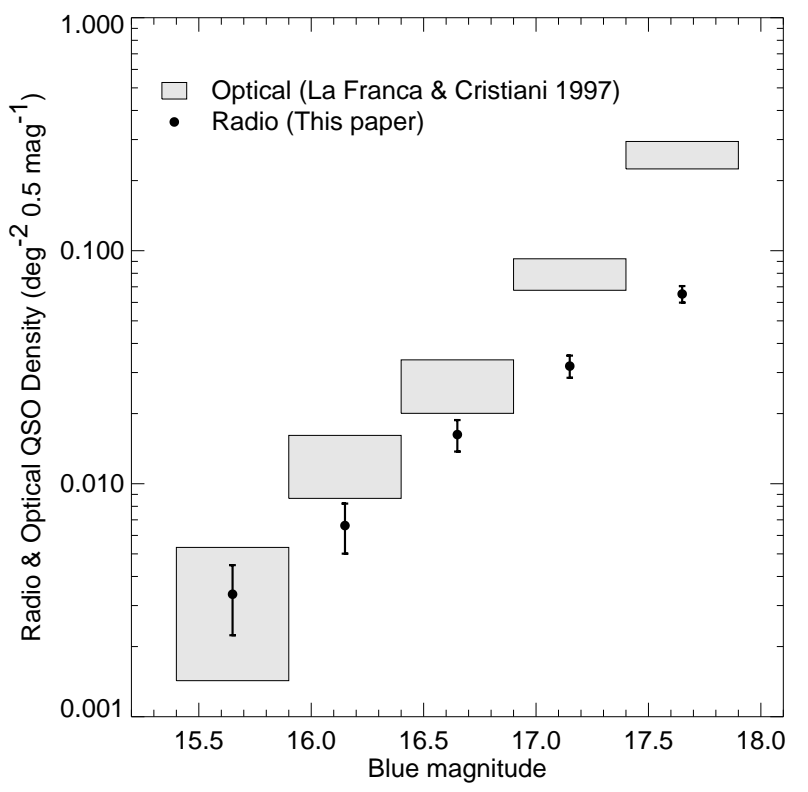

FIG. 13.- Density of FBQS quasars on the sky as a function of extinction-corrected blue magnitude. Shaded boxes show the density of all optically selected quasars from La Franca \& Cristiani (1997). The redshift range of FBQS quasars is restricted to $0.3 \leq z \leq 2.2$ to match the optical counts. The surface density of FBQS quasars is very similar to optically selected samples for $B<16$ mag, indicating that FIRST detects most optical quasars and that the FBQS and optical samples are similarly incomplete for bright objects. At $B=18$ mag the FBQS sky density drops to $\sim 25 \%$ of the optically selected quasar density.

and $\mathrm{J} 142703.6+270940(z=1.170)$. The spectra of these objects are remarkable; they are often difficult even to recognize as quasars. The emission lines are almost completely masked by absorption, and the colors of these objects are quite red. Such objects would be very unlikely to be recognized in any existing optically selected survey, so we really have no idea how common they may be among radio-quiet quasars.

In total there are 29 definite or tentative BAL quasars among the 636 FBQS quasars. This is comparable to the percentage of BAL quasars in optically selected samples (Foltz et al. 1990). The properties of the FBQS BAL quasar sample are discussed further by Becker et al. 1999.

Of course, the disadvantage of a radio-selected survey is that we can find only quasars with radio emission above the FIRST $1 \mathrm{mJy}$ limit, but as discussed above these constitute a significant minority (25\%) of all quasars brighter than $E=17.8$. The FIRST survey is sufficiently deep that we detect many radiointermediate and even radio-quiet quasars; only radio-silent quasars are not represented in the sample. This is most apparent in the number counts (Fig. 13), which are very similar for FBQS and optically selected samples for the brightest objects. It is the combination of large optical quasar surveys with the FBQS that may be most productive for studying quasar physics.

\subsection{No Radio-Loud/Radio-Quiet Dichotomy?}

Figure 14 shows the distribution of the radio-loudness parameter $R^{*}$ versus redshift. An $R^{*}$ of 10 is generally taken as the divide between radio-loud and radio-quiet objects (Stocke et al. 1992). It is clear that over the entire range of observed redshifts, the FBQS is reaching well into the radio-quiet population and that there is no obvious deficit of quasars at the boundary. Previous studies, in contrast, have always found a bimodal distribution in $R^{*}$ with a dearth of quasars in the range $3 \lesssim R^{*} \lesssim 100$ (e.g., Miller, Peacock, \& Mead 1990; Stocke et al. 1992).

The inadequacy of previous surveys in finding these radio- transition quasars is well illustrated in Figures 15(a) and 15(b), which show the histogram of the number of quasars versus $R^{*}$ compared with the distribution for previously known quasars. Although the FBQS shows a sharp increase in the number of quasars for $R^{*}<100$, this population is actually decreasing among the ranks of previously known quasars. The vast majority $(85 \%)$ of FBQS quasars with $1<R^{*}<100$ are newly discovered, while those with more extreme $R^{*}$ values in both directions are well-represented in existing samples.

Figure 15(a) also shows a histogram for previously known quasars that includes $R^{*}$ upper limits for all the Véron quasars (Véron-Cetty \& Véron 1998) that fit the FBQS criteria (including sky area and brightness) but that are not detected by the FIRST survey. This distribution is certainly bimodal; however, the FBQS quasars fill the gap and there is no clear evidence for bimodality in the combined FBQS and radio-quiet Véron distribution.

Conceivably this is the result of selection effects in the FBQS, though we have no plausible explanation for how our sample selection could eliminate a gap in the $R^{*}$ distribution. A definitive conclusion on the actual distribution of quasar radioloudness awaits full analysis and modeling of the sample and its selection effects.

\subsection{Summary}

This paper purposely defers most of the scientific studies that can be based on this new sample of quasars in order to make the data available to the community more quickly. Clearly the value of this database will be greatly enhanced with complementary data at other radio frequencies as well as in the X-ray, IR, and UV bands. The FBQS will serve as a vital bridge between the traditional radio-loud and radio-quiet quasar samples. The literature is full of characteristics such as line ratios, X-ray spectral indices, and broad absorption lines that appear to depend on radio-loudness. With the new sample, we can begin to explore the radio-loudness transition zone. 


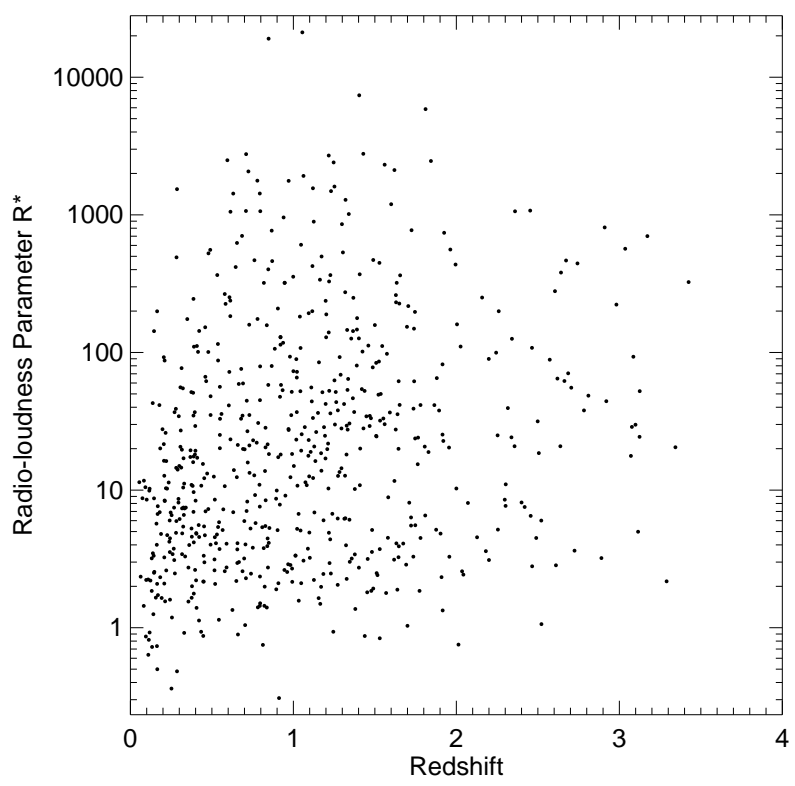

FIG. 14.- Radio-optical ratio $R^{*}$ (rest-frame ratio of the $5 \mathrm{GHz}$ radio flux density to the $2500 \mathrm{~A}$ optical flux; Stocke et al. 1992) versus redshift for FBQS quasars.

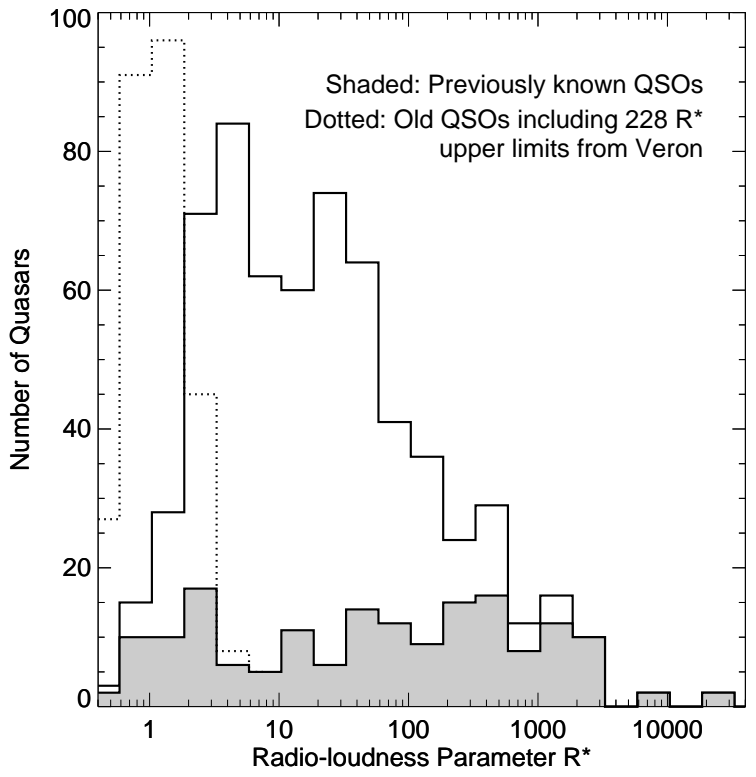

(a)

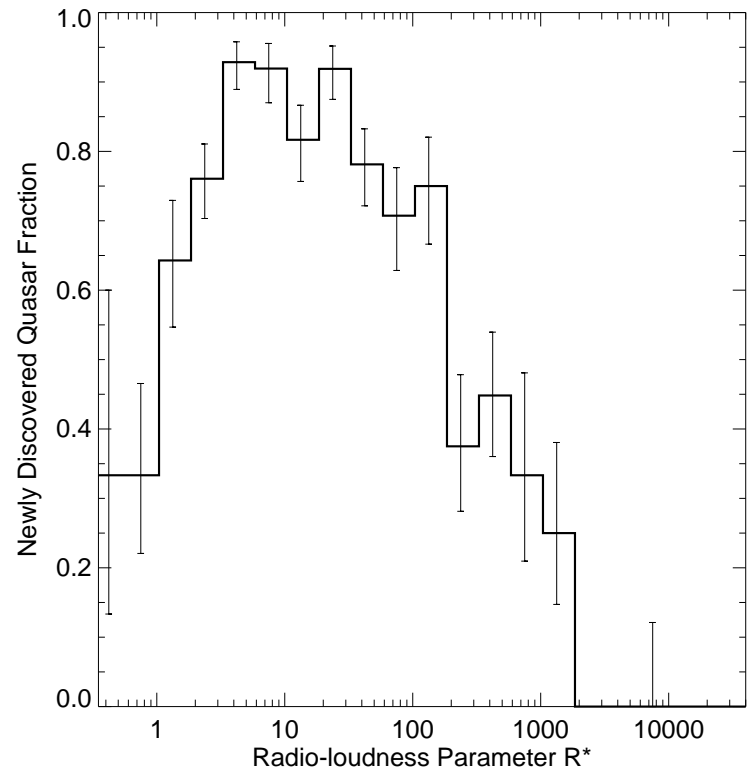

(b)

FIG. 15.- (a) Histogram of radio-optical ratio $R^{*}$ (Stocke et al. 1992) for FBQS quasars. Shaded: previously known quasars. The dotted histogram includes $R^{*}$ upper limits for Véron catalog objects in the FBQS area but not detected by FIRST. The Véron quasars (shaded plus dotted histograms) show a bimodal distribution of $R^{*}$, with a dip around $R^{*}=3-30$, but the FBQS quasar counts rise continuously through that region and show no obviously evidence for bimodality. (b) Fraction of quasars that were newly discovered versus $R^{*}$. The FBQS is increasing the number of known objects in the radio-quiet/radio-loud transition region $\left(R^{*}=1-100\right)$ by a large factor. 
The results in this paper are based on the 1997 version of the FIRST radio catalog, which covered 2682 square degrees of the north Galactic cap. As this is written in mid-1999, data have been collected that extend the area covered by FIRST to 6000 square degrees, and the FBQS candidate list will soon stand at $\sim 2700 ; \sim 1500$ of those candidates will eventually be confirmed as quasars (assuming that the telescope time allocation committees continue to be kind to us.) At that point the FBQS catalog will be one of the largest uniformly selected quasar surveys and will be an even more powerful tool for furthering our understanding of the quasar phenomenon.

Thanks to the referee, Todd Tripp, for many helpful suggestions, including an analysis of the FBQS/Hamburg match rate. We appreciate helpful discussions with Ed Moran on the classification of the emission line galaxies. Thanks to Hien Tran for computing the quasar redshifts from template correlations.
We acknowledge extensive use of the NASA/IPAC Extragalactic Database (NED), which is operated by the Jet Propulsion Laboratory, Caltech, under contract with the National Aeronautics and Space Administration. The success of the FIRST survey is in large measure due to the generous support of a number of organizations. In particular, we acknowledge support from the NRAO, the NSF (grants AST-98-02791 and AST98-02732), the Institute of Geophysics and Planetary Physics (operated under the auspices of the U.S. Department of Energy by Lawrence Livermore National Laboratory under contract No. W-7405-Eng-48), the Space Telescope Science Institute, NATO, the National Geographic Society (grant NGS No. 5393094), Columbia University, and Sun Microsystems. We appreciate the hospitality of the Institute of Astronomy, Cambridge University, which hosted visits enabling the writing of much of this paper.

\section{REFERENCES}

Barker, T., \& Cudworth, K. M. 1984, ApJ, 278, 610

Becker, R. H., White, R. L. \& Helfand, D. J. 1995, ApJ, 450, 559 (BWH95)

Becker, R. H., Gregg, M. D., Hook, I. M., McMahon, R. G., White, R. L., \& Helfand, D. J. 1997, ApJ, 479, L93

Becker, R. H., et al. 1999, in preparation

Brotherton, M. S., Gregg, M. D., Becker, R. H., Laurent-Muehleisen, S. A., White, R. L., \& Stanford, S. A. 1999, ApJ, submitted

Brotherton, M. S., Tran, H., et al. 1999, in preparation

Foltz, C. B., Chaffee, F. H., Hewett, P. C., Weymann, R. J., \& Morris, S. L. 1990, BAAS, 2, 806

Goldschmidt, P., Miller, L., La Franca, F., \& Cristiani, S. 1992, MNRAS, 256, $65 \mathrm{P}$

Green, R. F., Schmidt, M., \& Liebert, J. 1986, ApJS, 61, 305

Gregg, M., D., Becker, R. H., White, R. L., Helfand, D. J., McMahon, R. G., \& Hook, I. M. 1996, AJ, 112, 407 (Paper I)

Hagen, H.-J., Engels, D., \& Reimers, D. 1999, A\&AS, 134, 483

Helfand, D. J., Schnee, S., Becker, R. H., White, R. L., \& McMahon, R. G. 1999a, AJ, 117, 1568

Helfand, D. J., Willman, B., White, R. L., Becker, R. H., \& McMahon, R. G. 1999b, in preparation

Hewett, P. C., Foltz, C. B., \& Chaffee, F. H. 1995, AJ, 109, 1498 (LBQS)

Heath, D., Kasif, S., \& Salzberg, S. 1996, in Cognitive Technology: In Search of a Humane Interface, eds. B. Gorayska \& J. Mey (Amsterdam: Elsevier), p. 305

Hook, I. M., McMahon, R. G., Patnaik, A. R., Brown, I. W. A., Wilkinson, P. N., Irwin, M. J., \& Hazard, C. 1995, MNRAS, 273, 63L

Hook, I. M., Becker, R. H., McMahon, R. G., \& White, R. L. 1998, MNRAS, 297,1115

La Franca, F., \& Cristiani, S. 1997, AJ, 113, 1517

Laurent-Muehleisen, S. A., Kollgaard, R. I., Ciardullo, R., Feigelson, E. D., Brinkmann, W., \& Siebert, J. 1998, ApJS, 118, 127

Laurent-Muehleisen, S. A., et al. 1999, in preparation

Marchã, M. J. M., Browne, I. W. A., Impey, C. D., \& Smith, P. S., 1996, MNRAS, 281, 425
McMahon, R. G., \& Irwin, M. J. 1992, in Digitised Optical Sky Surveys, eds. H. T. MacGillivray and E. B. Thomson (Dordrecht: Kluwer), p. 417 McMahon, R. G., et al. 1999, in preparation

Miller, L., Peacock, J. A., \& Mead, A. R. G. 1990, MNRAS, 244, 207

Minkowski, R., \& Abell, G. 1963, in Stars and Stellar Systems, Vol. III, Basic Astronomical Data, ed. K. Aa. Strand (Chicago: Univ. of Chicago Press), 481

Moshir, M. M., et al. 1992, Explanatory Supplement to the IRAS Faint Source Survey, Version 2, JPL D-10015 (Pasadena: JPL)

Murthy, S. K., Kasif, S., \& Salzberg, S. 1994, Journal of Artificial Intelligence Research, 2, 1

Oke, J. B., Cohen, J. G., Carr, M., et al. 1995, PASP, 107, 375

Osterbrock, D. E., \& Pogge, R. W. 1985, ApJ, 297, 166

Osterbrock, D. E. 1989, Astrophysics of Gaseous Nebulae and Active Galactic Nuclei (Mill Valley California: University Science Books), p. 346

Pennington, R. L., Humphreys, R. M., Odewahn, S. C., Zumach, W., \& Thurmes, P. M. 1993, PASP, 105, 521

Salzberg, S., Chandar, R., Ford, H., Murthy, S. K., \& White, R. L. 1995, PASP, 107,279

Schechter, P. L., Gregg, M. D., Becker, R. H., Helfand, D. J., \& White, R. L. 1998, AJ, 115, 1371

Schlegel, D. J., Finkbeiner, D. P., \& Davis, M. 1998, ApJ, 500, 525

Stocke, J. T., et al. 1991, ApJS, 76, 813

Stocke, J. S., Morris, S. L., Weymann, R. J., \& Foltz, C. B. 1992, ApJ, 396, 487

Véron-Cetty, M.-P., \& Véron, P. 1998, A Catalogue of Quasars and Active Nuclei (8th Edition), European Southern Observatory Scientific Report, 18, 1

Voges, W. et al. 1996, IAU Circ., 6420

Weymann, R. J., Morris, S. L., Foltz, C. B., \& Hewett, P. C. 1991, ApJ, 373, 23

White, R. L. 1997, in Statistical Challenges in Modern Astronomy II, eds. G. J. Babu \& E. D. Feigelson (New York: Springer), p. 135

White, R. L., Becker, R. H., Helfand, D. J., \& Gregg, M. D. 1997, ApJ, 475, 479 (WBHG97)

Wisotzki, L., Koehler, T., Groote, D., \& Reimers, D. 1996, A\&AS, 115, 227 
TABLE 2

FIRST BRIGHT QUASAR CATALOG

\begin{tabular}{|c|c|c|c|c|c|c|c|c|c|c|c|c|c|c|}
\hline $\begin{array}{l}\text { RA } \\
\text { (1) }\end{array}$ & $\begin{array}{l}\text { Dec } \\
(2)\end{array}$ & $\begin{array}{l}\mathrm{Cl} \\
(3)\end{array}$ & $\begin{array}{c}E \\
(4)\end{array}$ & $\begin{array}{c}\mathrm{O} \\
(5)\end{array}$ & $\begin{array}{l}\text { O-E } \\
(6)\end{array}$ & $\begin{array}{c}\mathrm{A}(\mathrm{E}) \\
(7)\end{array}$ & $\begin{array}{l}\text { Sep } \\
(8)\end{array}$ & $\begin{array}{l}S_{p} \\
(9)\end{array}$ & $\begin{array}{c}S_{i} \\
(10)\end{array}$ & $\begin{array}{c}M_{B} \\
(11)\end{array}$ & $\begin{array}{c}\log L_{R} \\
\text { (12) }\end{array}$ & $\begin{array}{c}\log R^{*} \\
\text { (13) }\end{array}$ & $\begin{array}{c}\mathrm{z} \\
(14)\end{array}$ & $\begin{array}{l}\text { Notes } \\
\text { (15) }\end{array}$ \\
\hline 070732.951 & +38 2213.44 & $\mathrm{gE}$ & 17.19 & 17.10 & -0.09 & 0.17 & 0.45 & 66.80 & 218.76 & -25.8 & 33.2 & 2.42 & 0.579 & o \\
\hline 070806.412 & +364456.80 & $\mathrm{gE}$ & 16.80 & 17.63 & 0.83 & 0.17 & 0.25 & 15.80 & 15.78 & -23.8 & 31.5 & 1.54 & 0.297 & \\
\hline 071044.341 & +422054.75 & $*$ & 16.86 & 17.19 & 0.33 & 0.19 & 0.29 & 266.98 & 300.35 & -27.4 & 33.9 & 2.53 & 1.163 & o \\
\hline 071309.447 & +365606.60 & * & 15.80 & 15.80 & -0.00 & 0.17 & 0.38 & 71.90 & 93.10 & -26.8 & 32.7 & 1.55 & 0.492 & $\mathrm{o}, \mathrm{X}$ \\
\hline 071340.291 & +38 2040.08 & $*$ & 14.62 & 14.98 & 0.36 & 0.14 & 0.46 & 10.43 & 10.78 & -24.4 & 30.6 & 0.34 & 0.123 & $\mathrm{o}, \mathrm{X}$ \\
\hline 071424.813 & +35 3439.70 & $*$ & 17.31 & 17.51 & 0.20 & 0.16 & 0.06 & 1506.51 & 1533.45 & -27.9 & 34.9 & 3.33 & 1.620 & o \\
\hline 071734.467 & +291613.39 & $*$ & 17.17 & 17.44 & 0.27 & 0.13 & 0.82 & 2.18 & 2.25 & -27.0 & 31.8 & 0.51 & 1.101 & P1 \\
\hline 071919.414 & +330709.65 & $*$ & 17.76 & 18.78 & 1.02 & 0.15 & 0.37 & 302.28 & 329.81 & -24.9 & 33.6 & 3.25 & 0.779 & \\
\hline 071921.358 & +421250.86 & * & 17.15 & 17.44 & 0.29 & 0.20 & 0.08 & 7.28 & 7.83 & -27.0 & 32.3 & 1.06 & 1.074 & \\
\hline 072105.179 & +245617.21 & $\mathrm{gO}$ & 17.39 & 17.79 & 0.40 & 0.18 & 0.43 & 4.43 & 4.44 & -24.1 & 31.1 & 1.04 & 0.375 & \\
\hline 072201.260 & +372228.68 & * & 17.01 & 17.65 & 0.64 & 0.16 & 0.34 & 145.26 & 147.74 & -27.8 & 33.9 & 2.36 & 1.630 & O(APS),o \\
\hline 072418.492 & +415914.40 & $*$ & 17.65 & 18.71 & 1.05 & 0.24 & 0.16 & 7.89 & 7.90 & -26.6 & 32.6 & 1.52 & 1.552 & E(APS),O(APS),LoBAL \\
\hline 072438.348 & +342621.08 & $\mathrm{gO}$ & 17.65 & 17.97 & 0.33 & 0.15 & 0.05 & 22.76 & 23.58 & -24.4 & 32.1 & 1.82 & 0.460 & \\
\hline 072526.450 & +291341.73 & $*$ & 17.19 & 17.76 & 0.57 & 0.12 & 0.41 & 1.64 & 0.97 & -27.2 & 31.8 & 0.48 & 1.346 & P1 \\
\hline 072550.600 & +281905.83 & $*$ & 17.01 & 17.85 & 0.85 & 0.13 & 0.19 & 37.99 & 38.85 & -28.8 & 33.8 & 1.79 & 2.663 & \\
\hline 072609.182 & +401002.30 & $\mathrm{gE}$ & 17.76 & 19.72 & 1.96 & 0.14 & 0.77 & 97.17 & 102.36 & -21.6 & 32.3 & 3.19 & 0.286 & \\
\hline 072637.031 & +375908.90 & $*$ & 17.50 & 17.76 & 0.26 & 0.16 & 0.33 & 1.11 & 0.88 & -24.3 & 30.6 & 0.42 & 0.397 & \\
\hline 072831.661 & +402615.85 & $*$ & 15.13 & 15.26 & 0.14 & 0.13 & 0.24 & 16.96 & 16.79 & -28.0 & 32.2 & 0.57 & 0.656 & LoBAL \\
\hline 072928.475 & +252451.99 & * & 17.25 & 17.81 & 0.56 & 0.15 & 0.09 & 6.83 & 6.47 & -28.4 & 32.9 & 1.04 & 2.303 & \\
\hline 072939.071 & +353613.50 & $*$ & 17.80 & 18.17 & 0.37 & 0.14 & 0.55 & 1.17 & 1.26 & -24.8 & 31.0 & 0.61 & 0.590 & ? \\
\hline 072952.300 & +304645.06 & $*$ & 16.87 & 17.30 & 0.43 & 0.18 & 0.34 & 1.48 & 1.17 & -22.5 & 29.9 & 0.41 & 0.147 & P1 \\
\hline 073047.098 & +263409.89 & * & 17.41 & 17.74 & 0.32 & 0.16 & 0.44 & 1.81 & 2.15 & -25.0 & 31.1 & 0.68 & 0.531 & E(APS \\
\hline 073521.205 & +264744.51 & $\mathrm{gE}$ & 16.47 & 17.77 & 1.30 & 0.13 & 0.42 & 1.66 & 1.20 & -23.1 & 30.3 & 0.63 & 0.239 & \\
\hline 073600.363 & +383629.52 & $*$ & 17.29 & 18.13 & 0.85 & 0.16 & 0.31 & 1.24 & 1.42 & -27.0 & 31.8 & 0.55 & 1.482 & \\
\hline 073623.157 & +392617.38 & $\mathrm{gE}$ & 14.31 & 15.22 & 0.90 & 0.14 & 0.14 & 3.50 & 3.63 & -24.1 & 30.1 & -0.03 & 0.118 & $\mathrm{o}, \mathrm{X}$ \\
\hline 073919.561 & +304314.83 & $*$ & 17.74 & 17.83 & 0.09 & 0.12 & 0.33 & 4.72 & 4.90 & -25.8 & 31.8 & 1.04 & 0.792 & \\
\hline 074004.775 & +341205.29 & $*$ & 17.62 & 18.22 & 0.59 & 0.12 & 0.25 & 1.42 & 1.30 & -26.7 & 31.7 & 0.60 & 1.323 & \\
\hline 074011.663 & +253728.03 & $*$ & 16.87 & 17.18 & 0.31 & 0.13 & 0.50 & 5.70 & 5.73 & -27.6 & 32.3 & 0.79 & 1.272 & \\
\hline 074103.692 & +422513.27 & $*$ & 17.36 & 17.39 & 0.02 & 0.14 & 0.26 & 1.05 & 0.95 & -24.6 & 30.6 & 0.25 & 0.389 & \\
\hline 074110.698 & +311200.31 & $*$ & 16.44 & 16.50 & 0.06 & 0.13 & 0.26 & 2051.49 & 2071.27 & -26.6 & 34.3 & 3.15 & 0.631 & o \\
\hline 074205.862 & +351503.98 & $*$ & 17.80 & 18.86 & 1.06 & 0.19 & 0.13 & 24.11 & 25.18 & -26.1 & 33.0 & 2.10 & 1.355 & \\
\hline 074334.486 & +310906.29 & * & 17.32 & 17.56 & 0.24 & 0.13 & 0.93 & 1.71 & 1.02 & -28.2 & 32.1 & 0.37 & 1.909 & \\
\hline 074352.023 & +271239.79 & $*$ & 15.72 & 15.64 & -0.08 & 0.12 & 0.22 & 1.02 & 0.81 & -25.4 & 30.2 & -0.44 & 0.252 & I \\
\hline $0744 \quad 10.474$ & +411733.85 & $*$ & 17.63 & 17.78 & 0.15 & 0.12 & 0.60 & 51.66 & 61.83 & -27.4 & 33.5 & 2.05 & 1.460 & \\
\hline 074412.042 & +295906.69 & $*$ & 17.67 & 17.70 & 0.04 & 0.12 & 0.14 & 93.72 & 94.90 & -27.8 & 33.8 & 2.19 & 1.697 & P1 \\
\hline 074417.467 & +3753 17.22 & * & 17.22 & 17.50 & 0.28 & 0.14 & 0.30 & 1113.79 & 1244.87 & -26.9 & 34.5 & 3.28 & 1.062 & o \\
\hline 074505.924 & +261409.91 & $*$ & 16.19 & 17.15 & 0.96 & 0.10 & 0.21 & 32.83 & 33.62 & -27.6 & 33.0 & 1.56 & 1.227 & \\
\hline 074541.659 & +314256.52 & $*$ & 15.26 & 15.30 & 0.04 & 0.18 & 0.56 & 614.67 & 632.74 & -27.1 & 33.5 & 2.18 & 0.459 & $\mathrm{o}, \mathrm{X}$ \\
\hline 074559.311 & +331334.52 & * & 17.59 & 17.64 & 0.06 & 0.14 & 0.10 & 98.52 & 126.82 & -25.4 & 33.0 & 2.40 & 0.608 & o \\
\hline 074604.904 & +292250.92 & $\mathrm{gE}$ & 17.28 & 17.78 & 0.51 & 0.11 & 0.54 & 1.62 & 1.31 & -25.0 & 31.0 & 0.57 & 0.547 & P1 \\
\hline 074618.722 & +2306 23.34 & * & 17.23 & 17.78 & 0.54 & 0.14 & 0.22 & 3.07 & 2.68 & -28.0 & 32.4 & 0.71 & 1.876 & \\
\hline 074920.007 & +234104.37 & * & 17.07 & 18.27 & 1.20 & 0.17 & 0.26 & 2.06 & 1.48 & -23.1 & 30.6 & 0.91 & 0.295 & \\
\hline 074927.911 & +415242.20 & $*$ & 17.15 & 18.13 & 0.98 & 0.13 & 0.08 & 15.29 & 15.20 & -28.8 & 33.5 & 1.48 & 3.099 & \\
\hline 074948.175 & +345443.76 & $\mathrm{gO}$ & 15.62 & 16.07 & 0.45 & 0.14 & 0.86 & 1.31 & 0.82 & -23.5 & 29.7 & -0.14 & 0.133 & $\mathrm{o}, \mathrm{X}$ \\
\hline 075047.362 & +413033.11 & * & 17.02 & 17.18 & 0.15 & 0.12 & 0.03 & 2.06 & 2.22 & -27.5 & 31.8 & 0.39 & 1.184 & \\
\hline 075112.354 & +291937.30 & $*$ & 15.29 & 15.55 & 0.27 & 0.11 & 1.15 & 1.00 & 1.16 & -28.5 & 31.3 & -0.51 & 0.912 & P1 \\
\hline 075245.634 & +261735.12 & * & 15.93 & 16.82 & 0.89 & 0.09 & 0.67 & 1.13 & 1.27 & -21.7 & 29.3 & 0.16 & 0.082 & $\mathrm{o}, \mathrm{X}$ \\
\hline 075448.829 & +303355.13 & * & 17.11 & 17.54 & 0.43 & 0.15 & 0.47 & 44.75 & 46.47 & -26.2 & 32.8 & 1.90 & 0.796 & $\mathrm{P} 1, \mathrm{X}$ \\
\hline 075800.047 & +392029.09 & $\mathrm{gO}$ & 13.90 & 13.87 & -0.03 & 0.18 & 0.13 & 10.80 & 11.56 & -25.0 & 30.4 & -0.06 & 0.095 & $\mathrm{o}, \mathrm{X}, \mathrm{I}$ \\
\hline 075813.948 & +262418.75 & $\mathrm{gE}$ & 17.13 & 17.87 & 0.74 & 0.14 & 0.18 & 13.72 & 13.91 & -27.9 & 33.0 & 1.40 & 1.915 & \\
\hline 075928.298 & +301028.97 & * & 17.18 & 17.74 & 0.56 & 0.16 & 0.65 & 161.38 & 182.20 & -26.5 & 33.6 & 2.55 & 0.999 & P1 \\
\hline 080409.242 & +385348.71 & * & 16.82 & 17.90 & 1.08 & 0.14 & 0.12 & 2.88 & 2.68 & -22.7 & 30.5 & 0.92 & 0.211 & I \\
\hline 080413.671 & +251634.07 & * & 17.39 & 18.09 & 0.70 & 0.08 & 0.36 & 3.48 & 3.68 & -28.2 & 32.6 & 0.89 & 2.302 & \\
\hline 080849.182 & +400918.87 & * & 17.50 & 18.06 & 0.56 & 0.14 & 0.70 & 1.95 & 1.49 & -25.8 & 31.5 & 0.72 & 0.853 & \\
\hline 080856.647 & +4052 44.92 & $\mathrm{gE}$ & 17.80 & 18.77 & 0.97 & 0.14 & 0.67 & 589.58 & 608.29 & -26.3 & 34.4 & 3.44 & 1.429 & o \\
\hline 080901.332 & +275341.67 & * & 17.17 & 17.58 & 0.40 & 0.10 & 0.68 & 1.17 & 1.67 & -27.7 & 31.9 & 0.40 & 1.511 & HiBAL \\
\hline 080906.230 & +291235.58 & * & 17.06 & 17.59 & 0.53 & 0.13 & 0.15 & 21.48 & 21.87 & -27.6 & 33.0 & 1.52 & 1.476 & P1 \\
\hline 080906.466 & +413932.68 & * & 16.47 & 17.28 & 0.81 & 0.13 & 0.32 & 2.21 & 2.45 & -27.4 & 31.9 & 0.47 & 1.222 & o \\
\hline 081054.197 & +232145.27 & * & 16.44 & 16.86 & 0.42 & 0.13 & 0.24 & 12.84 & 14.35 & -26.6 & 32.2 & 1.13 & 0.731 & \\
\hline 081130.749 & +390410.26 & * & 17.43 & 18.00 & 0.57 & 0.13 & 0.73 & 1.62 & 1.82 & -27.4 & 32.0 & 0.59 & 1.647 & \\
\hline 081148.342 & +375645.43 & * & 17.08 & 17.76 & 0.69 & 0.11 & 0.15 & 1.76 & 1.60 & -27.4 & 31.9 & 0.50 & 1.456 & \\
\hline 081240.683 & +320808.51 & $*$ & 17.66 & 18.55 & 0.89 & 0.12 & 0.39 & 18.32 & 18.41 & -28.1 & 33.4 & 1.74 & 2.705 & $\operatorname{dmpL} \alpha$ \\
\hline 081409.215 & +323731.88 & * & 17.73 & 18.15 & 0.42 & 0.13 & 0.44 & 126.85 & 136.64 & -25.7 & 33.3 & 2.60 & 0.846 & o \\
\hline 081415.054 & +412323.44 & * & 17.54 & 18.21 & 0.67 & 0.12 & 0.06 & 5.15 & 4.65 & -26.6 & 32.3 & 1.16 & 1.295 & \\
\hline 081446.213 & +223645.58 & $*$ & 17.52 & 17.93 & 0.41 & 0.11 & 0.64 & 1.64 & 2.63 & -23.7 & 30.8 & 0.87 & 0.326 & \\
\hline 081520.657 & +273616.90 & * & 15.97 & 16.40 & 0.44 & 0.09 & 0.00 & 3.62 & 3.15 & -27.6 & 31.8 & 0.32 & 0.908 & $\mathrm{o}, \mathrm{X}$ \\
\hline 081738.344 & +242330.05 & * & 16.40 & 16.96 & 0.56 & 0.11 & 0.31 & 5.02 & 4.45 & -24.3 & 31.0 & 0.77 & 0.282 & $\mathrm{X}$ \\
\hline 081740.150 & +232731.60 & $\mathrm{gE}$ & 16.13 & 16.82 & 0.69 & 0.14 & 0.02 & 10.02 & 10.10 & -27.1 & 32.3 & 0.94 & 0.894 & \\
\hline 081849.231 & +38 3416.44 & $\mathrm{gE}$ & 16.99 & 17.82 & 0.83 & 0.10 & 0.92 & 1.00 & 2.08 & -22.2 & 30.1 & 0.76 & 0.160 & \\
\hline 082107.619 & +310751.40 & $*$ & 16.58 & 16.98 & 0.40 & 0.11 & 0.20 & 85.41 & 89.98 & -29.6 & 34.1 & 1.81 & 2.620 & o \\
\hline 082346.003 & +410404.07 & $*$ & 16.97 & 17.07 & 0.10 & 0.12 & 0.55 & 1.01 & 1.30 & -26.6 & 31.3 & 0.16 & 0.794 & \\
\hline
\end{tabular}


TABLE 2-Continued

\begin{tabular}{|c|c|c|c|c|c|c|c|c|c|c|c|c|c|c|}
\hline $\begin{array}{l}\text { RA } \\
(1)\end{array}$ & $\begin{array}{l}\text { Dec } \\
(2)\end{array}$ & $\begin{array}{l}\mathrm{Cl} \\
(3)\end{array}$ & $\begin{array}{c}\mathrm{E} \\
(4)\end{array}$ & $\begin{array}{c}\mathrm{O} \\
(5)\end{array}$ & $\begin{array}{c}\text { O-E } \\
(6)\end{array}$ & $\begin{array}{c}\mathrm{A}(\mathrm{E}) \\
(7)\end{array}$ & $\begin{array}{l}\text { Sep } \\
(8)\end{array}$ & $\begin{array}{l}S_{p} \\
(9)\end{array}$ & $\begin{array}{c}S_{i} \\
(10)\end{array}$ & $\begin{array}{c}M_{B} \\
(11)\end{array}$ & $\begin{array}{c}\log L_{R} \\
\text { (12) }\end{array}$ & $\begin{array}{c}\log R^{*} \\
(13)\end{array}$ & $\begin{array}{c}\mathrm{Z} \\
(14)\end{array}$ & $\begin{array}{l}\text { Notes } \\
(15)\end{array}$ \\
\hline 082358.226 & 364954.14 & $\mathrm{gE}$ & 17.74 & 18.44 & 0.71 & 0.14 & 0.24 & 13.64 & 13.64 & -26.5 & 32.7 & 1.67 & 1.365 & \\
\hline 06.241 & +334245.45 & $*$ & 17.53 & 18.25 & 0.72 & 0.11 & 0.54 & 1.93 & 1.08 & -23.3 & 30.6 & 0.87 & 0.317 & \\
\hline 082438.971 & +405708.19 & $*$ & 17.09 & 17.24 & 0.14 & 0.13 & 0.37 & 87.01 & 174.72 & -25.8 & 33.2 & 2.38 & 0.613 & \\
\hline 082455.475 & +391641.76 & $*$ & 17.50 & 17.74 & 0.24 & 0.12 & 0.10 & 1403.96 & 1456.09 & -27.0 & 34.7 & 3.43 & 1.217 & $\mathrm{o}, \mathrm{X}$ \\
\hline 082806.853 & +393540.30 & $\mathrm{gE}$ & 17.64 & 17.95 & 0.31 & 0.10 & 0.42 & 4.59 & 5.11 & -25.6 & 31.8 & 1.11 & 0.760 & 0 \\
\hline 083052.076 & +241059.85 & $*$ & 16.30 & 17.09 & 0.79 & 0.09 & 0.07 & 835.48 & 885.66 & -27.0 & 34.2 & 2.98 & 0.940 & $\mathrm{o}, \mathrm{X}$ \\
\hline 083225.342 & +370736.64 & $\mathrm{gE}$ & 15.47 & 16.56 & 1.10 & 0.10 & 0.11 & 11.78 & 11.73 & -22.2 & 30.4 & 1.02 & 0.092 & $\mathrm{o}, \mathrm{X}$ \\
\hline 083246.974 & +285312.68 & $\mathrm{gE}$ & 17.04 & 17.84 & 0.79 & 0.11 & 0.94 & 1.64 & 1.73 & -22.9 & 30.3 & 0.67 & 0.226 & $\mathrm{P} 1, \mathrm{X}$ \\
\hline 083431.610 & +240145.90 & $*$ & 17.76 & 17.77 & 0.01 & 0.10 & 0.10 & 27.22 & 29.88 & -27.3 & 33.1 & 1.73 & 1.420 & \\
\hline 083535.820 & +245940.65 & $*$ & 15.76 & 16.04 & 0.28 & 0.08 & 0.53 & 1.84 & 1.76 & -25.6 & 30.7 & -0.04 & 0.330 & o \\
\hline 083622.895 & +272852.49 & $*$ & 17.49 & 17.42 & -0.07 & 0.11 & 0.62 & 300.48 & 304.39 & -26.2 & 33.6 & 2.67 & 0.761 & o \\
\hline 083636.885 & +412554.87 & $*$ & 17.25 & 17.81 & 0.55 & 0.10 & 0.13 & 424.23 & 443.13 & -27.1 & 34.2 & 2.93 & 1.298 & $0, X$ \\
\hline 083740.261 & +245423.00 & $*$ & 17.40 & 17.80 & 0.41 & 0.11 & 0.29 & 422.63 & 446.21 & -26.7 & 34.1 & 2.95 & 1.125 & o \\
\hline 084044.457 & +363328.41 & $*$ & 16.20 & 17.53 & 1.34 & 0.10 & 0.43 & 1.63 & 1.00 & -27.2 & 31.7 & 0.39 & 1.230 & FeLoBAL \\
\hline 084118.087 & +354439.02 & $*$ & 16.18 & 16.88 & 0.70 & 0.10 & 0.21 & 6.01 & 5.66 & -28.7 & 32.6 & 0.65 & 1.780 & o \\
\hline 084310.772 & +395345.13 & $*$ & 17.24 & 18.11 & 0.87 & 0.09 & 0.16 & 2.57 & 2.17 & -24.0 & 31.0 & 0.92 & 0.400 & $\mathrm{O}(\mathrm{A}$ \\
\hline 084437.984 & +412432.12 & $\mathrm{gE}$ & 16.40 & 17.17 & 0.77 & 0.08 & 0.84 & 1.58 & 1.38 & -25.5 & 31.0 & 0.32 & 0.520 & \\
\hline 084715.181 & +3831 10.13 & $*$ & 17.70 & 18.86 & 1.15 & 0.08 & 0.33 & 184.89 & 185.49 & -28.2 & 34.6 & 2.85 & 3.172 & \\
\hline 084716.046 & +373217.85 & $*$ & 17.23 & 17.46 & 0.24 & 0.09 & 0.29 & 1.69 & 1.20 & -24.9 & 30.9 & 0.48 & 0.454 & $\mathrm{o}, \mathrm{X}$ \\
\hline 084832.032 & +423108.46 & $*$ & 17.13 & 17.73 & 0.60 & 0.08 & 0.18 & 1.71 & 1.55 & -24.1 & 30.7 & 0.60 & 0.361 & \\
\hline 084902.577 & +300234.86 & $*$ & 16.35 & 16.54 & 0.20 & 0.10 & 0.66 & 1.02 & 1.26 & -26.7 & 31.1 & -0.05 & 0.660 & o \\
\hline 085317.834 & +281349.95 & $*$ & 17.22 & 17.61 & 0.39 & 0.11 & 0.11 & 71.73 & 72.97 & -26.4 & 33.1 & 2.11 & 21 & 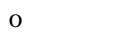 \\
\hline 085721.069 & +36384 & $*$ & 17.40 & 17.59 & 0.19 & 0.08 & 0.82 & 1.09 & 0.78 & -24.8 & 30.7 & 0.34 & 0.450 & \\
\hline 085726.954 & +331317.24 & $*$ & 17.08 & 17.43 & 0.36 & 0.07 & 0.29 & 20.69 & 21.29 & -28.9 & 33.4 & 1.38 & 2.338 & \\
\hline 090007.973 & +364610.77 & $*$ & 16.80 & 17.04 & 0.24 & 0.10 & 0.30 & 2.67 & 2.38 & -25.6 & 31.2 & 0.50 & 0.516 & \\
\hline 090033.493 & +421546.48 & $*$ & 16.70 & 17.69 & 0.99 & 0.07 & 0.64 & 1.52 & 1.71 & -29.4 & 32.6 & 0.34 & 3.290 & $\mathrm{E}(A$ \\
\hline 090239.895 & +395726.77 & $*$ & 17.73 & 18.55 & 0.82 & 0.05 & 0.07 & 32.00 & 32.35 & -28.4 & 33.8 & 1.97 & 3.086 & \\
\hline & +31201 & $*$ & 17.57 & 17.92 & 0.35 & 0.06 & & 1.73 & 1.26 & -24.8 & 0 & 0.66 & 18 & \\
\hline 090325.620 & +224148.10 & $*$ & 17.63 & 19.38 & 1.75 & 0.08 & 0.40 & 1.29 & 0.84 & -22.0 & 30.4 & 1.15 & 0.290 & I \\
\hline 090429.630 & +281932.83 & $*$ & 16.86 & 16.87 & 0.02 & 0.08 & 0.03 & 34.28 & 39.24 & -27.6 & 33.0 & 1.52 & 1.120 & o \\
\hline 090444.337 & +233354.05 & $*$ & 16.87 & 17.20 & 0.33 & 0.08 & 0.10 & 105.35 & 107.31 & -29.0 & 34.1 & 2.00 & 2.244 & o \\
\hline 090541.757 & +284928.22 & $*$ & 17.46 & 17.64 & 0.18 & 0.08 & 0.05 & 190.44 & 195.06 & -27.1 & 33.8 & 2.52 & 1.219 & \\
\hline 090838.808 & +324620.47 & $\mathrm{gE}$ & 17.54 & 17.86 & 0.32 & 0.05 & 0.61 & 1.25 & 0.88 & -23.2 & 30.3 & 0.53 & 0.259 & \\
\hline 090947.293 & +302403.01 & $*$ & 17.66 & 17.57 & -0.09 & 0.07 & 0.50 & 8.57 & 17.49 & -26.1 & 32.4 & 1.49 & 0.784 & \\
\hline 090947.827 & +312443.41 & $*$ & 16.64 & 17.56 & 0.93 & 0.06 & 0.26 & 1.81 & 1.50 & -23.6 & 30.5 & 0.57 & 0.266 & \\
\hline 091044.902 & +261253.71 & $*$ & 17.78 & 19.26 & 1.48 & 0.09 & 0.11 & 7.84 & 7.46 & -27.6 & 33.1 & 1.65 & 2.920 & $\mathrm{HiB}$ \\
\hline 091054.184 & +375915.11 & $*$ & 17.22 & 17.20 & -0.02 & 0.06 & 0.04 & 250.93 & 265.93 & -28.9 & 34.4 & 2.40 & 2.158 & o,new $z$ \\
\hline 091055.239 & +253921.58 & $*$ & 17.38 & 18.18 & 0.81 & 0.10 & 0.11 & 202.60 & 207.22 & -28.5 & 34.5 & 2.65 & 2.743 & \\
\hline 091206.976 & +391146.25 & $*$ & 17.63 & 18.04 & 0.41 & 0.04 & 0.21 & 16.98 & 17.80 & -27.5 & 33.1 & 1.59 & 1.737 & \\
\hline 091247.806 & +285406.56 & $\mathrm{gE}$ & 16.74 & 17.43 & 0.70 & 0.07 & 0.37 & 1.22 & 0.88 & -22.9 & 30.0 & 0.37 & 0.183 & $\mathrm{P} 1, \mathrm{X}$ \\
\hline 091328.260 & +394444.17 & $*$ & 17.16 & 17.99 & 0.83 & 0.05 & 0.19 & 2.06 & 2.09 & -27.4 & 32.1 & 0.65 & 1.580 & HiBAL \\
\hline 091806.472 & +302406.81 & $*$ & 17.63 & 17.95 & 0.31 & 0.06 & 0.66 & 2.00 & 2.01 & -24.8 & 31.1 & 0.73 & 0.538 & \\
\hline 091858.154 & +232555.43 & $*$ & 16.80 & 17.20 & 0.40 & 0.17 & 0.14 & 26.70 & 27.00 & -26.1 & 32.5 & 1.54 & 0.688 & \\
\hline 091954.289 & +291408.55 & $*$ & 17.46 & 17.64 & 0.17 & 0.06 & 0.11 & 8.10 & 8.33 & -25.8 & 32.0 & 1.20 & 0.720 & $\mathrm{P} 1$ \\
\hline 092104.452 & +302031.04 & $\mathrm{gE}$ & & & 1.45 & 0.06 & & 3.99 & 4.33 & -28.0 & 33.0 & 1.31 & 3.344 & \\
\hline 092211.008 & +223641.65 & $*$ & 17.64 & 17.99 & 0.35 & 0.10 & 0.39 & 8.83 & 9.41 & -27.5 & 32.7 & 1.30 & 1.645 & \\
\hline 092335.466 & +312409.01 & $*$ & 17.32 & 17.56 & 0.24 & 0.05 & 0.39 & 4.11 & 3.35 & -26.8 & 32.0 & 0.83 & 1.032 & \\
\hline 092425.030 & +354712.79 & $*$ & & & 0.26 & 0.04 & 0.24 & & 13.27 & -26.9 & .7 & 1.48 & 1.344 & \\
\hline 092554.719 & +400414.18 & $*$ & 17.09 & 17.23 & 0.14 & 0.03 & 0.20 & 9.16 & 9.30 & -25.2 & 31.7 & 1.12 & 0.471 & $\mathrm{X}$ \\
\hline 092913.940 & +375742.99 & $*$ & 17.61 & 17.91 & 0.30 & 0.04 & 0.12 & 43.19 & 43.43 & -27.9 & 33.5 & 1.91 & 1.915 & \\
\hline 093255.471 & +284036.52 & $*$ & 16.61 & 16.66 & 0.05 & 0.05 & 0.34 & 1.40 & 0.94 & -26.1 & 0 & 0.06 & 0.543 & o \\
\hline 093311.637 & +351627.90 & $*$ & 17.49 & 18.03 & 0.53 & 0.04 & 0.12 & 4.85 & 4.97 & -26.5 & 32.1 & 1.09 & 1.121 & \\
\hline 093324.620 & +222238.11 & $*$ & 17.78 & 18.25 & 0.47 & 0.08 & 0.11 & 4.26 & 3.91 & -23.7 & 31.2 & 1.20 & 0.385 & \\
\hline 093329.584 & +245648.73 & $*$ & 17.64 & 19.56 & 1.92 & 0.06 & 0.38 & 2.20 & 2.44 & -27.4 & 32.7 & 1.25 & 3.071 & \\
\hline 093337.298 & +284532.24 & $*$ & 17.45 & 18.52 & 1.07 & 0.05 & 0.25 & 119.85 & 120.71 & -28.7 & 34.4 & 2.51 & 3.425 & \\
\hline 093403.978 & +315331.47 & $*$ & 17.43 & 17.83 & 0.39 & 0.05 & 0.24 & 4.68 & 4.41 & -28.5 & 32.8 & 0.88 & 2.419 & HiBAL \\
\hline 093407.306 & +354238.21 & $*$ & 17.19 & 17.89 & 0.70 & 0.04 & 0.16 & 3.87 & 5.75 & -26.6 & 32.2 & 1.10 & 1.097 & \\
\hline 093447.566 & +290230.65 & $*$ & 17.79 & 17.83 & 0.03 & 0.05 & 0.14 & 20.93 & 21.55 & -26.7 & 32.8 & 1.64 & 1.113 & \\
\hline 093531.668 & +354100.97 & $*$ & 17.08 & 17.56 & 0.48 & 0.04 & 0.67 & 1.54 & 2.10 & -25.0 & 31.1 & 0.60 & 0.492 & \\
\hline 093603.893 & +320709.31 & $*$ & 17.17 & 17.33 & 0.16 & 0.05 & 0.18 & 27.45 & 28.30 & -27.2 & 32.9 & 1.56 & 1.151 & \\
\hline 093621.508 & +392132.00 & $\mathrm{gE}$ & 16.95 & 17.96 & 1.01 & 0.04 & 0.32 & 27.15 & 28.51 & -22.6 & 31.5 & 1.94 & 0.210 & \\
\hline 093703.014 & +361537.35 & $\mathrm{gE}$ & 16.38 & 17.44 & 1.06 & 0.04 & 0.14 & 3.60 & 3.21 & -22.8 & 30.4 & 0.84 & 0.180 & $\mathrm{X}, \mathrm{I}$ \\
\hline 093704.050 & +293704.91 & $*$ & 17.28 & 17.63 & 0.35 & 0.05 & 0.21 & 2.45 & 2.65 & -24.7 & 31.1 & 0.74 & 0.452 & $\mathrm{P} 1$ \\
\hline 094146.901 & +353036.48 & $*$ & 17.65 & 18.32 & 0.67 & 0.03 & 0.30 & 1.12 & 0.93 & -22.0 & 30.0 & 0.68 & 0.188 & \\
\hline 094149.668 & +412104.57 & $*$ & 17.72 & 18.73 & 1.01 & 0.05 & 0.06 & 62.16 & 63.30 & -25.0 & 33.0 & 2.51 & 0.819 & \\
\hline 094258.054 & +401014.22 & $*$ & 16.77 & 17.57 & 0.80 & 0.05 & 0.61 & 1.73 & 2.02 & -26.7 & 31.7 & 0.52 & 1.013 & \\
\hline 094311.136 & +293804.83 & $*$ & 17.23 & 17.82 & 0.60 & 0.06 & 0.23 & 1.04 & 0.91 & -27.8 & 31.8 & 0.27 & 1.774 & $\mathrm{P} 1$ \\
\hline 094324.328 & +283540.19 & $*$ & 17.78 & 18.14 & 0.36 & 0.05 & 0.11 & 1.39 & 2.12 & -24.3 & 31.0 & 0.85 & 0.461 & \\
\hline 094418.876 & +233119.98 & $*$ & 17.22 & 18.19 & 0.97 & 0.07 & 0.22 & 11.16 & 11.86 & -26.0 & 32.4 & 1.54 & 0.987 & \\
\hline 094439.002 & +225209.96 & $*$ & 17.24 & 18.54 & 1.30 & 0.08 & 0.65 & 1.25 & 1.83 & -20.7 & 29.7 & 1.00 & 0.116 & \\
\hline 094602.299 & +274407.04 & $*$ & 16.89 & 17.65 & 0.76 & 0.06 & 0.33 & 3.54 & 3.63 & -27.9 & 32.4 & 0.74 & 1.748 & HiBAL \\
\hline
\end{tabular}


White, Becker, et al.

TABLE 2-Continued

\begin{tabular}{|c|c|c|c|c|c|c|c|c|c|c|c|c|c|c|}
\hline $\begin{array}{l}\text { RA } \\
\text { (1) }\end{array}$ & $\begin{array}{l}\text { Dec } \\
(2)\end{array}$ & $\begin{array}{l}\mathrm{Cl} \\
(3)\end{array}$ & $\begin{array}{c}E \\
(4)\end{array}$ & $\begin{array}{c}\mathrm{O} \\
(5)\end{array}$ & $\begin{array}{c}\text { O-E } \\
(6)\end{array}$ & $\begin{array}{c}\mathrm{A}(\mathrm{E}) \\
(7)\end{array}$ & $\begin{array}{c}\text { Sep } \\
(8)\end{array}$ & $\begin{array}{l}S_{p} \\
(9)\end{array}$ & $\begin{array}{c}S_{i} \\
(10)\end{array}$ & $\begin{array}{c}M_{B} \\
(11)\end{array}$ & $\begin{array}{c}\log L_{R} \\
\text { (12) }\end{array}$ & $\begin{array}{c}\log R^{*} \\
\text { (13) }\end{array}$ & $\begin{array}{c}\mathrm{Z} \\
(14)\end{array}$ & $\begin{array}{l}\text { Notes } \\
(15)\end{array}$ \\
\hline 094610.958 & 322326.23 & * & 16.92 & 7.21 & 0.28 & .05 & 0.41 & 1.41 & 1.56 & -24.9 & 30.8 & 0.34 & 0.403 & \\
\hline 44.719 & +414304.33 & $*$ & 17.18 & 18.15 & 0.97 & 0.04 & 0.39 & 26.33 & 31.69 & -26.1 & 32.9 & 1.95 & 018 & o \\
\hline 094647.090 & +232751.25 & $*$ & 17.26 & 18.29 & 1.03 & 0.08 & 0.31 & 1.01 & 1.18 & -28.3 & 32.2 & 0.45 & 2.611 & \\
\hline 094712.039 & +345119.60 & $*$ & 16.86 & 17.58 & 0.71 & 0.03 & 0.28 & 1.04 & 1.20 & -27.6 & 31.7 & 0.26 & 1.453 & \\
\hline 094855.357 & +403944.67 & $*$ & 16.98 & 17.57 & 0.59 & 0.04 & 0.08 & 1439.97 & 1537.07 & -27.2 & 34.7 & 3.38 & 1.247 & o \\
\hline 094927.680 & +314109.96 & $*$ & 15.79 & 16.73 & 0.94 & 0.06 & 0.34 & 28.12 & 28.52 & -24.7 & 31.8 & 1.43 & 0.309 & I \\
\hline 095122.539 & +263514.11 & $*$ & 16.20 & 16.24 & 0.04 & 0.06 & 0.59 & 1.48 & 2.03 & -28.5 & 31.8 & -0.03 & 1.245 & grav \\
\hline 095159.986 & +220906.15 & $\mathrm{gO}$ & 16.85 & 17.63 & 0.78 & 0.10 & 0.26 & 1.16 & 1.11 & -23.1 & 30.1 & 0.42 & 0.220 & $X$ \\
\hline 095206.385 & +235245.27 & $\mathrm{gO}$ & 16.57 & 16.77 & 0.20 & 0.08 & 0.12 & 32.49 & 34.61 & -27.4 & 32.9 & 1.44 & 0.970 & \\
\hline 095227.207 & +224006.16 & $*$ & 16.67 & 17.17 & 0.50 & 0.08 & 0.67 & 18.13 & 19.83 & -27.5 & 32.8 & 1.34 & 1.213 & \\
\hline 095327.957 & +322551.62 & $*$ & 16.62 & 16.83 & 0.21 & 0.03 & 0.04 & 128.43 & 131.85 & -28.5 & 33.8 & 1.99 & 1.574 & o,new pos \\
\hline 095331.656 & +391729.74 & $*$ & 16.87 & 17.49 & 0.62 & 0.03 & 0.59 & 4.97 & 4.45 & -26.5 & 32.0 & 0.89 & 0.917 & \\
\hline 095537.936 & +333503.97 & $*$ & 17.03 & 17.16 & 0.13 & 0.04 & 0.12 & 35.73 & 36.61 & -29.3 & 33.7 & 1.50 & 2.499 & $\mathrm{o}, \mathrm{dn}$ \\
\hline 095555.663 & +351652.89 & $*$ & 17.13 & 17.36 & 0.23 & 0.03 & 0.36 & 9.28 & 9.71 & -28.0 & 32.7 & 1.07 & 1.620 & \\
\hline 095649.873 & +251515.96 & $*$ & 16.11 & 16.93 & 0.82 & 0.10 & 0.25 & 1041.77 & 1069.98 & -26.5 & 34.1 & 3.03 & 0.709 & o \\
\hline 095707.367 & +235625.32 & $*$ & 17.63 & 18.47 & 0.84 & 0.09 & 0.01 & 136.10 & 140.49 & -27.4 & 34.1 & 2.64 & 1.995 & $\mathrm{HiB}$ \\
\hline 095820.940 & +322402.17 & $*$ & 15.74 & 15.56 & -0.18 & 0.04 & 0.34 & 1204.19 & 1228.71 & -27.2 & 33.9 & 2.56 & 0.533 & o \\
\hline 095905.550 & +240614.52 & $*$ & 16.95 & 18.05 & 1.10 & 0.10 & 0.10 & 14.55 & 14.85 & -27.1 & 32.8 & 1.54 & 1.469 & \\
\hline 095930.513 & +300734.57 & $*$ & 17.60 & 17.46 & -0.13 & 0.05 & 0.10 & 3.41 & 3.40 & -25.3 & 31.4 & 0.77 & 0.544 & \\
\hline 100029.158 & +275212.08 & $*$ & 17.64 & 17.98 & 0.33 & 0.05 & 0.16 & 79.49 & 82.16 & -26.7 & 33.4 & 2.28 & 1.202 & \\
\hline 100208.123 & +345353.78 & $*$ & 16.79 & 18.21 & 1.42 & 0.03 & 0.19 & 5.58 & 5.30 & -22.3 & 30.7 & 1.33 & 0.205 & \\
\hline 100254.540 & +324038.93 & $*$ & 16.97 & 16.89 & -0.08 & 0.04 & 0.50 & 9.09 & 9.00 & -26.9 & 32.1 & 0.93 & 30 & $\mathrm{O}$ \\
\hline 100318 & +272734.51 & $*$ & 17.32 & 17.84 & 0.52 & 0.06 & 0.53 & 6.75 & 6.76 & -27.0 & 32.4 & 1.13 & 1.283 & \\
\hline 100410.197 & +372848.08 & $*$ & 17.59 & 17.77 & 0.18 & 0.04 & 0.80 & 1.15 & 0.96 & -25.7 & 31.1 & 0.39 & 0.740 & \\
\hline 100442.904 & +242226.02 & $*$ & 16.72 & 17.53 & 0.80 & 0.10 & 0.21 & 19.92 & 19.79 & -27.3 & 32.8 & 1.48 & 1.255 & \\
\hline 100445.746 & +222519.38 & $*$ & 17.00 & 18.02 & 1.02 & 0.11 & 0.15 & 33.93 & 36.95 & -26.2 & 32.9 & 1.97 & 0.979 & $o, X$ \\
\hline 100507.905 & +341424.12 & $\mathrm{gE}$ & 16.36 & 16.90 & 0.54 & 0.03 & 0.34 & 3.40 & 2.66 & -23.1 & 30.3 & 0.60 & 0.162 & $\mathrm{X}$ \\
\hline 100 & & $*$ & & & 1.18 & 0.03 & & 1.29 & 1.07 & & 30.5 & 0.53 & & $\mathrm{X}$ \\
\hline 100642.628 & +270115.30 & $*$ & 17.17 & 17.29 & 0.11 & 0.08 & 0.12 & 13.83 & 13.30 & -25.5 & 32.0 & 1.30 & 0.549 & \\
\hline 100841.247 & +362319.26 & $*$ & 16.93 & 17.85 & 0.93 & 0.03 & 0.07 & 33.01 & 34.77 & -29.1 & 33.8 & 1.72 & 3.125 & $\mathrm{dmpl}$ \\
\hline 101000.711 & +300321.52 & $*$ & 16.13 & 16.48 & 0.35 & 0.07 & 0.10 & 1.54 & 0.99 & -24.6 & 30.4 & 0.07 & 0.255 & $o, X$ \\
\hline 101027.525 & +413238.91 & $*$ & 16.09 & 16.23 & 0.14 & 0.04 & 0.29 & 258.74 & 340.26 & -26.8 & 33.5 & 2.26 & 0.613 & $\mathrm{o}, \mathrm{X}$ \\
\hline 101211.434 & +330925.96 & $*$ & 17.26 & 17.30 & 0.04 & 0.05 & 0.73 & 172.71 & 196.33 & -28.9 & 34.3 & 2.30 & 2.260 & o \\
\hline 101244.373 & +355347.90 & $*$ & 17.45 & 18.07 & 0.62 & 0.03 & 0.40 & 26.45 & 27.61 & -26.2 & 32.8 & 1.86 & 1.022 & \\
\hline 101302.287 & +352605.47 & $*$ & 17.49 & 18.00 & 0.51 & 0.03 & 0.19 & 208.11 & 208.02 & -28.6 & 34.5 & 2.58 & 2.642 & \\
\hline 101325.429 & +221229.03 & $*$ & 17.00 & 17.67 & 0.67 & 0.07 & 0.27 & 1.09 & 1.08 & -23.5 & 30.3 & 0.39 & 0.273 & $\mathrm{X}, \mathrm{I}$ \\
\hline 101353.421 & +244916.37 & $*$ & 15.92 & 16.65 & 0.73 & 0.11 & 0.37 & 500.27 & 517.34 & -28.8 & 34.5 & 2.51 & 1.634 & $0, X$ \\
\hline 101505.682 & +360452.63 & $*$ & 17.16 & 17.70 & 0.54 & 0.03 & 0.08 & 29.51 & 29.85 & -26.1 & 32.7 & 1.76 & 0.846 & \\
\hline 101752.685 & +324234.81 & $*$ & 17.73 & 17.61 & -0.12 & 0.04 & 0.26 & 3.28 & 3.20 & -28.4 & 32.5 & 0.66 & 2.127 & \\
\hline 101810.996 & +354239.71 & $*$ & 17.43 & 18.09 & 0.66 & 0.03 & 0.56 & 571.31 & 582.58 & -26.6 & 34.3 & 3.17 & 1.231 & o \\
\hline 101825.444 & +380532.64 & $*$ & 17.43 & 17.23 & -0.20 & 0.04 & 0.42 & 32.69 & 34.78 & -24.8 & 32.1 & 1.71 & 0.385 & $o, X$ \\
\hline 101858.491 & & $\mathrm{gO}$ & & 16.61 & 0.86 & 0.04 & 0.85 & 2.27 & 2.44 & -22.5 & 29.8 & 0.35 & 0.109 & \\
\hline 102041.170 & +395810.87 & $*$ & 17.74 & 18.73 & 0.99 & 0.04 & 0.88 & 2.71 & 2.38 & -25.0 & 31.6 & 1.14 & 0.826 & \\
\hline 102117.509 & +343721.93 & $*$ & 16.82 & 17.05 & 0.23 & 0.04 & 0.35 & 317.10 & 392.03 & -28.0 & 34.2 & 2.57 & 1.407 & o \\
\hline 102156.523 & +300140.62 & $*$ & & & & 0.07 & & 1.43 & 1.41 & & 32.4 & 70 & 3.115 & \\
\hline 102230.318 & +304105.19 & $*$ & 16.80 & 17.46 & 0.66 & 0.07 & 0.22 & 907.01 & 919.28 & -27.4 & 34.5 & 3.11 & 1.320 & $\mathrm{o}$ \\
\hline 102237.416 & +393150.54 & $*$ & 16.40 & 17.15 & 0.75 & 0.04 & 0.26 & 41.28 & 57.53 & -25.9 & 32.7 & 1.86 & 0.607 & o \\
\hline 102311.597 & & $*$ & & & 0.61 & 0.04 & & 807.88 & 1078.67 & & 34.6 & 21 & 52 & $o, X$ \\
\hline 102322.305 & +363912.60 & $*$ & 17.56 & 18.08 & 0.52 & 0.03 & 0.16 & 8.29 & 8.31 & -24.7 & 31.7 & 1.40 & 0.549 & \\
\hline 102333.511 & +395312.52 & $*$ & 17.35 & 17.98 & 0.63 & 0.03 & 0.90 & 62.13 & 65.13 & -26.9 & 33.4 & 2.16 & 1.330 & \\
\hline 102553.597 & +401243.49 & $\mathrm{gE}$ & 16.15 & 17.11 & 0.96 & 0.03 & 0.65 & 1.07 & 0.82 & -25.0 & 30.6 & 0.14 & 0.406 & $\mathrm{o}, \mathrm{X}$ \\
\hline 102617.468 & +303643.01 & $*$ & 17.09 & 18.33 & 1.24 & 0.05 & 0.08 & 1.74 & 1.82 & -23.4 & 30.7 & 0.87 & 0.340 & P1,I \\
\hline 102837.788 & +262359.08 & $*$ & 17.48 & 18.31 & 0.84 & 0.06 & 0.52 & 3.03 & 2.54 & -27.7 & 32.4 & 0.91 & 2.071 & \\
\hline 103045.236 & +255522.06 & $*$ & 16.16 & 17.14 & 0.97 & 0.05 & 0.42 & 47.56 & 49.15 & -26.2 & 32.7 & 1.78 & 0.691 & \\
\hline 103059.104 & +310255.74 & $\mathrm{gE}$ & 15.37 & 16.27 & 0.90 & 0.05 & 0.11 & 58.74 & 63.45 & -24.0 & 31.7 & 1.62 & 0.178 & $\mathrm{o}, \mathrm{X}$ \\
\hline 103110.647 & +395322.81 & $*$ & 17.73 & 18.66 & 0.92 & 0.03 & 0.53 & 2.45 & 2.03 & -25.8 & 31.8 & 1.04 & 1.082 & LoB \\
\hline 103303.732 & +411606.32 & $*$ & 17.65 & 18.44 & 0.79 & 0.04 & 0.12 & 406.68 & 432.65 & -26.1 & 34.1 & 3.19 & 1.120 & o \\
\hline 103359.483 & +355509.48 & $\mathrm{gE}$ & 16.02 & 16.45 & 0.43 & 0.06 & 0.86 & 2.06 & 2.23 & -23.7 & 30.2 & 0.24 & 0.169 & I \\
\hline 103510.984 & +351019.51 & $*$ & 17.33 & 17.10 & -0.23 & 0.06 & 0.28 & 23.03 & 22.88 & -28.8 & 33.3 & 1.31 & 1.955 & \\
\hline 103511.672 & +340625.10 & $*$ & 17.68 & 17.71 & 0.03 & 0.05 & 0.96 & 34.87 & 36.63 & -25.6 & 32.6 & 1.88 & 0.680 & \\
\hline 103551.191 & +375641.50 & $*$ & 16.41 & 16.29 & -0.12 & 0.05 & 1.09 & 52.76 & 54.26 & -28.9 & 33.4 & 1.39 & 1.508 & \\
\hline 103641.942 & +250236.63 & $*$ & 16.87 & 17.57 & 0.70 & 0.06 & 0.07 & 97.26 & 118.51 & -28.4 & 34.0 & 2.20 & 2.004 & \\
\hline 103648.486 & +370307.71 & $\mathrm{gO}$ & 17.79 & 17.50 & -0.29 & 0.04 & 0.77 & 26.23 & 26.71 & -27.9 & 33.2 & 1.56 & 1.592 & \\
\hline 103848.147 & +372924.39 & $*$ & 17.10 & 17.14 & 0.04 & 0.05 & 0.93 & 17.47 & 17.46 & -26.3 & 32.3 & 1.32 & 0.730 & \\
\hline 103853.331 & +392150.43 & $\mathrm{gE}$ & 16.93 & 18.92 & 1.99 & 0.06 & 0.93 & 1.19 & 1.43 & -18.7 & 29.0 & 1.06 & 0.055 & \\
\hline 103859.593 & +422742.04 & $*$ & 17.46 & 18.67 & 1.22 & 0.04 & 0.23 & 2.76 & 2.44 & -22.0 & 30.5 & 1.21 & 0.220 & \\
\hline 103941.964 & +242240.33 & $*$ & 17.63 & 18.64 & 1.00 & 0.06 & 0.27 & 53.61 & 116.94 & -26.0 & 33.5 & 2.70 & 1.173 & \\
\hline 104229.180 & +381111.32 & $*$ & 17.55 & 17.96 & 0.41 & 0.06 & 0.19 & 11.39 & 11.80 & -28.6 & 33.2 & 1.32 & 2.638 & \\
\hline 104459.591 & +365605.39 & $*$ & 16.51 & 17.23 & 0.72 & 0.04 & 0.30 & 14.61 & 15.00 & -26.2 & 32.2 & 1.30 & 0.701 & FeLoBAL \\
\hline 104556.863 & +271759.54 & $*$ & 17.17 & 17.86 & 0.68 & 0.09 & 0.09 & 8.74 & 9.86 & -26.7 & 32.4 & 1.32 & 1.128 & \\
\hline 104620.192 & +342708.32 & $*$ & 17.05 & 17.24 & 0.19 & 0.05 & 0.16 & 35.95 & 35.93 & -27.4 & 33.1 & 1.63 & 1.198 & \\
\hline
\end{tabular}


TABLE 2-Continued

\begin{tabular}{|c|c|c|c|c|c|c|c|c|c|c|c|c|c|c|}
\hline $\begin{array}{l}\text { RA } \\
\text { (1) }\end{array}$ & $\begin{array}{l}\text { Dec } \\
\text { (2) }\end{array}$ & $\begin{array}{l}\mathrm{Cl} \\
\text { (3) }\end{array}$ & $\begin{array}{c}E \\
(4)\end{array}$ & $\begin{array}{c}\mathrm{O} \\
(5)\end{array}$ & $\begin{array}{l}\text { O-E } \\
\text { (6) }\end{array}$ & $\begin{array}{c}\mathrm{A}(\mathrm{E}) \\
(7)\end{array}$ & $\begin{array}{l}\text { Sep } \\
(8)\end{array}$ & $\begin{array}{l}S_{p} \\
(9)\end{array}$ & $\begin{array}{c}S_{i} \\
(10)\end{array}$ & $\begin{array}{c}M_{B} \\
(11)\end{array}$ & $\begin{array}{c}\log L_{R} \\
\text { (12) }\end{array}$ & $\begin{array}{c}\log R^{*} \\
\text { (13) }\end{array}$ & $\begin{array}{c}\mathrm{Z} \\
(14)\end{array}$ & $\begin{array}{l}\text { Notes } \\
(15)\end{array}$ \\
\hline 926 & 06.36 & $*$ & 16.66 & 17.60 & 0.93 & 0.08 & 0.71 & 1.56 & 1.67 & -24.8 & 30.9 & 0.52 & 0.450 & \\
\hline 104820.129 & +302627.10 & * & 17.54 & 18.22 & 0.68 & 0.09 & 0.19 & 2.66 & 2.49 & -23.8 & 31.0 & 0.99 & 0.388 & \\
\hline 104832.411 & +290643.82 & * & 17.47 & 18.65 & 1.17 & 0.07 & 0.01 & 32.86 & 33.01 & -24.8 & 32.6 & 2.20 & 0.729 & \\
\hline 105027.466 & +350920.58 & * & 17.72 & 17.81 & 0.08 & 0.05 & 0.22 & 1.51 & 0.97 & -26.6 & 31.6 & 0.49 & 1.062 & \\
\hline 105113.634 & +38 5015.42 & $\mathrm{gE}$ & 17.40 & 18.43 & 1.03 & 0.04 & 0.83 & 4.95 & 5.53 & -22.2 & 30.8 & 1.42 & 0.216 & $X$ \\
\hline 105203.168 & +240505.03 & $*$ & 17.00 & 17.95 & 0.95 & 0.10 & 0.21 & 10.35 & 10.27 & -24.1 & 31.6 & 1.47 & 0.398 & \\
\hline 105250.084 & +335505.00 & * & 16.70 & 16.89 & 0.19 & 0.05 & 0.23 & 12.81 & 13.41 & -28.2 & 32.8 & 1.04 & 1.408 & \\
\hline 105403.223 & +415256.83 & * & 17.44 & 18.20 & 0.76 & 0.04 & 0.83 & 1.00 & 1.29 & -26.2 & 31.5 & 0.57 & 1.089 & \\
\hline 105426.370 & +263632.69 & $*$ & 17.78 & 18.47 & 0.69 & 0.06 & 0.44 & 1.73 & 1.53 & -27.7 & 32.3 & 0.71 & 2.255 & \\
\hline 105426.573 & +2703 17.48 & $*$ & 17.44 & 18.07 & 0.63 & 0.06 & 0.43 & 47.40 & 52.59 & -27.0 & 33.3 & 2.10 & 1.402 & \\
\hline 105427.150 & +253600.33 & $*$ & 16.93 & 18.38 & 1.45 & 0.07 & 0.58 & 2.99 & 3.02 & -28.0 & 32.6 & 0.91 & 2.400 & LoBAL \\
\hline 105518.452 & +392905.36 & $\mathrm{gE}$ & 16.82 & 17.56 & 0.74 & 0.04 & 0.28 & 2.79 & 3.15 & -23.5 & 30.7 & 0.82 & 0.253 & \\
\hline 105528.808 & +312411.65 & $*$ & 16.99 & 18.67 & 1.68 & 0.08 & 0.26 & 9.07 & 9.06 & -23.9 & 31.7 & 1.68 & 0.492 & \\
\hline 105543.932 & +294917.91 & $*$ & 17.37 & 17.79 & 0.42 & 0.05 & 0.77 & 13.99 & 15.18 & -27.3 & 32.8 & 1.45 & 1.430 & \\
\hline 105611.799 & +315616.34 & $*$ & 17.58 & 18.00 & 0.42 & 0.07 & 0.84 & 10.40 & 11.10 & -27.2 & 32.7 & 1.39 & 1.510 & \\
\hline 105648.171 & +370450.78 & $*$ & 16.82 & 17.37 & 0.55 & 0.05 & 0.78 & 19.43 & 20.90 & -24.6 & 31.8 & 1.54 & 0.387 & \\
\hline 105705.160 & +311907.79 & $*$ & 17.53 & 18.49 & 0.96 & 0.07 & 0.33 & 13.84 & 14.87 & -26.4 & 32.8 & 1.73 & 1.332 & \\
\hline 105834.374 & +313656.28 & $*$ & 17.48 & 18.55 & 1.07 & 0.08 & 0.57 & 1.45 & 1.29 & -27.9 & 32.3 & 0.65 & 2.490 & \\
\hline 105951.914 & +405113.70 & $*$ & 17.44 & 17.75 & 0.31 & 0.03 & 0.70 & 107.39 & 117.78 & -27.8 & 33.9 & 2.29 & 1.746 & \\
\hline 110001.081 & +231412.53 & $*$ & 16.99 & 17.81 & 0.82 & 0.05 & 0.92 & 11.82 & 15.23 & -25.0 & 32.0 & 1.55 & 0.559 & \\
\hline 110026.082 & +230341.31 & $*$ & 17.45 & 18.19 & 0.74 & 0.04 & 0.51 & 1.57 & 1.61 & -27.3 & 32.0 & 0.61 & 1.674 & \\
\hline 110211.882 & +284041.49 & $\mathrm{gE}$ & 16.50 & 17.45 & 0.96 & 0.07 & 0.55 & 46.17 & 48.36 & -28.1 & 33.5 & 1.79 & 1.741 & P1 \\
\hline 110223.364 & +223920.72 & $*$ & 17.21 & 18.78 & 1.57 & 0.06 & 0.17 & 2.05 & 1.79 & -23.6 & 31.0 & 1.09 & 0.453 & \\
\hline 110313.315 & +301442.29 & $*$ & 17.35 & 17.70 & 0.35 & 0.04 & 0.70 & 107.68 & 108.99 & -24.3 & 32.6 & 2.39 & 0.387 & $\mathrm{o}, \mathrm{X}$ \\
\hline 110340.267 & +372925.30 & $\mathrm{gE}$ & 15.66 & 17.20 & 1.53 & 0.04 & 0.39 & 5.33 & 5.42 & -21.1 & 29.8 & 0.94 & 0.074 & \\
\hline 110605.665 & +305108.72 & $*$ & 16.49 & 17.15 & 0.66 & 0.06 & 0.20 & 1.69 & 1.92 & -28.3 & 32.0 & 0.28 & 1.638 & P1 \\
\hline 110704.537 & +320630.06 & * & 16.56 & 17.18 & 0.62 & 0.07 & 0.21 & 3.03 & 2.82 & -23.8 & 30.6 & 0.65 & 0.243 & $\mathrm{o}$ \\
\hline 110838.321 & +25 5521.58 & $*$ & 17.71 & 17.91 & 0.21 & 0.05 & 0.23 & 10.87 & 10.31 & -25.5 & 32.1 & 1.43 & 0.718 & \\
\hline 110937.547 & +404203.26 & $\mathrm{gE}$ & 17.79 & 18.70 & 0.91 & 0.05 & 0.29 & 1.90 & 1.70 & -23.3 & 30.8 & 1.03 & 0.382 & \\
\hline 111040.226 & +301909.81 & $*$ & 17.31 & 17.98 & 0.67 & 0.06 & 0.41 & 20.08 & 22.77 & -27.3 & 33.1 & 1.69 & 1.522 & P1 \\
\hline 111050.517 & +232509.38 & $*$ & 17.62 & 18.37 & 0.76 & 0.04 & 0.49 & 1.14 & 0.88 & -25.5 & 31.3 & 0.62 & 0.849 & \\
\hline 111202.886 & +230038.12 & $*$ & 17.75 & 17.89 & 0.14 & 0.04 & 0.16 & 25.67 & 26.01 & -27.2 & 33.1 & 1.72 & 1.437 & \\
\hline 111223.301 & +240648.17 & $*$ & 17.55 & 18.25 & 0.70 & 0.04 & 0.17 & 15.32 & 17.27 & -26.4 & 32.7 & 1.71 & 1.178 & \\
\hline 111504.226 & +251100.51 & $*$ & 17.25 & 17.27 & 0.02 & 0.04 & 0.14 & 2.25 & 1.94 & -26.9 & 31.7 & 0.46 & 0.982 & \\
\hline 111549.856 & +223852.86 & $*$ & 17.72 & 18.17 & 0.45 & 0.04 & 0.16 & 7.75 & 7.17 & -27.5 & 32.7 & 1.28 & 1.829 & \\
\hline 111628.313 & +223119.75 & * & 17.33 & 17.65 & 0.32 & 0.04 & 0.49 & 2.39 & 2.21 & -26.2 & 31.6 & 0.65 & 0.842 & \\
\hline 111635.788 & +341914.41 & $*$ & 17.70 & 18.15 & 0.45 & 0.05 & 0.16 & 10.44 & 10.57 & -26.5 & 32.5 & 1.46 & 1.188 & \\
\hline 111903.290 & +385852.43 & $*$ & 17.78 & 18.12 & 0.35 & 0.05 & 0.43 & 11.81 & 11.75 & -25.4 & 32.1 & 1.55 & 0.730 & o,No spec \\
\hline 111930.318 & +222649.29 & $\mathrm{gE}$ & 17.06 & 17.31 & 0.24 & 0.05 & 0.18 & 90.58 & 92.75 & -24.9 & 32.6 & 2.16 & 0.422 & $\mathrm{X}$ \\
\hline 112111.119 & +3702 46.92 & $*$ & 17.40 & 18.20 & 0.80 & 0.05 & 0.22 & 100.54 & 100.76 & -27.2 & 33.8 & 2.42 & 1.629 & \\
\hline 112211.522 & +423709.78 & $*$ & 16.58 & 16.56 & -0.02 & 0.05 & 0.59 & 1.09 & 0.90 & -27.2 & 31.2 & -0.12 & 0.813 & \\
\hline 112220.462 & +312441.19 & $*$ & 17.08 & 18.19 & 1.11 & 0.04 & 0.19 & 12.64 & 12.87 & -26.9 & 328 & 1.54 & 1.448 & LoB \\
\hline 112241.467 & +303534.88 & $*$ & 16.35 & 16.73 & 0.38 & 0.04 & 0.36 & 10.06 & 10.02 & -28.9 & 32.8 & 0.82 & 1.809 & P1 \\
\hline 112434.169 & +322819.57 & $*$ & 17.34 & 18.03 & 0.69 & 0.06 & 0.39 & 24.72 & 38.13 & -27.2 & 33.3 & 1.93 & 1.525 & \\
\hline 112611.625 & +425245.96 & $*$ & 16.30 & 17.12 & 0.83 & 0.04 & 0.49 & 1.14 & 1.10 & -22.8 & 29.8 & 0.22 & 0.156 & \\
\hline 112736.394 & +265450.13 & $*$ & 16.19 & 16.72 & 0.53 & 0.05 & 0.37 & 2.18 & 1.86 & -25.2 & 30.8 & 0.30 & 0.378 & o \\
\hline 112751.065 & +401847.20 & $*$ & 17.06 & 17.45 & 0.39 & 0.04 & 0.11 & 12.85 & 13.17 & -26.3 & 32.3 & 1.31 & 0.829 & \\
\hline 112824.053 & +222420.56 & $*$ & 17.20 & 17.85 & 0.66 & 0.05 & 0.35 & 1.73 & 1.39 & -27.6 & 32.0 & 0.51 & 1.645 & \\
\hline 112851.697 & +232617.24 & * & 17.63 & 18.80 & 1.16 & 0.05 & 0.07 & 150.88 & 156.62 & -28.1 & 34.5 & 2.75 & 3.036 & o \\
\hline 112857.828 & +362250.74 & $*$ & 17.51 & 18.02 & 0.51 & 0.06 & 0.52 & 1.11 & 1.77 & -28.8 & 32.5 & 0.51 & 2.890 & $\mathrm{o}$ \\
\hline 112922.479 & +333049.74 & * & 17.43 & 17.93 & 0.50 & 0.05 & 0.03 & 6.21 & 6.00 & -26.7 & 32.3 & 1.14 & 1.172 & \\
\hline 112956.520 & +364919.20 & $\mathrm{gO}$ & 17.44 & 16.75 & -0.69 & 0.06 & 0.26 & 20.70 & 20.54 & -25.3 & 31.9 & 1.29 & 0.399 & \\
\hline 113004.732 & +411619.76 & * & 15.85 & 16.15 & 0.30 & 0.05 & 0.28 & 1.86 & 2.09 & -29.1 & 32.0 & -0.08 & 1.530 & o,new $z$ \\
\hline 113044.756 & +240102.35 & $*$ & 17.65 & 17.50 & -0.15 & 0.05 & 0.32 & 11.37 & 10.92 & -26.5 & 32.3 & 1.26 & 0.922 & \\
\hline 113109.214 & +263208.38 & $\mathrm{gE}$ & 15.45 & 16.18 & 0.73 & 0.05 & 0.93 & 2.00 & 2.72 & -24.8 & 30.6 & 0.20 & 0.243 & $\mathrm{o}, \mathrm{X}$ \\
\hline 113246.342 & +3740 56.43 & $*$ & 17.59 & 17.77 & 018 & 0.06 & 0.47 & 1.07 & 0.85 & -26.6 & 314 & 0.32 & 1.048 & \\
\hline 113303.859 & +370457.20 & $\mathrm{gO}$ & 17.13 & 17.59 & 0.45 & 0.06 & 0.41 & 16.15 & 17.82 & -27.3 & 32.8 & 1.45 & 1.295 & \\
\hline 113314.760 & +281159.65 & $*$ & 16.63 & 17.14 & 0.51 & 0.06 & 0.74 & 9.73 & 9.58 & -25.5 & 31.8 & 1.10 & 0.512 & $\mathrm{o}$ \\
\hline 113427.802 & +414721.70 & * & 16.67 & 17.10 & 0.44 & 0.06 & 0.53 & 3.34 & 2.89 & -26.7 & 31.7 & 0.58 & 0.818 & $\mathrm{X}$ \\
\hline 113448.669 & +354356.77 & $*$ & 17.48 & 17.68 & 0.20 & 0.06 & 0.58 & 1.28 & 0.96 & -25.7 & 31.1 & 0.41 & 0.688 & \\
\hline 113454.502 & +300526.48 & * & 17.21 & 17.14 & -0.07 & 0.07 & 0.30 & 306.09 & 847.49 & -25.9 & 33.9 & 3.02 & 0.614 & o \\
\hline 113457.636 & +255527.79 & * & 17.29 & 17.32 & 0.03 & 0.06 & 0.29 & 30.31 & 31.21 & -26.1 & 32.5 & 1.65 & 0.714 & \\
\hline 113636.791 & +390004.96 & $*$ & 15.64 & 16.06 & 0.42 & 0.07 & 0.33 & 5.38 & 4.89 & -27.6 & 31.9 & 0.37 & 0.795 & \\
\hline 113655.963 & +343236.76 & $\mathrm{gO}$ & 16.00 & 16.73 & 0.72 & 0.05 & 0.27 & 1.66 & 1.27 & -23.7 & 30.1 & 0.22 & 0.192 & \\
\hline 113709.464 & +390723.60 & $*$ & 17.58 & 18.27 & 0.68 & 0.07 & 0.73 & 1.15 & 1.66 & -26.0 & 31.6 & 0.72 & 1.023 & \\
\hline 113910.100 & +37 3211.49 & * & 16.47 & 17.15 & 0.67 & 0.05 & 0.12 & 1.91 & 1.81 & -28.1 & 32.0 & 0.28 & 1.490 & \\
\hline 114130.114 & +38 1351.31 & * & 17.07 & 17.41 & 0.34 & 0.06 & 0.04 & 2.33 & 2.17 & -26.9 & 31.7 & 0.52 & 1.013 & \\
\hline 114243.362 & +333025.49 & $*$ & 17.07 & 17.48 & 0.41 & 0.06 & 0.04 & 13.98 & 14.36 & -27.6 & 32.8 & 1.30 & 1.410 & \\
\hline 114337.893 & +231502.55 & * & 17.04 & 17.45 & 0.41 & 0.07 & 0.47 & 100.26 & 101.50 & -26.4 & 33.2 & 2.20 & 0.837 & \\
\hline 114549.498 & +290641.68 & * & 14.74 & 15.86 & 1.12 & 0.06 & 0.50 & 2.74 & 1.78 & -23.8 & 30.1 & 0.10 & 0.141 & P1 \\
\hline 114631.693 & +274624.07 & $*$ & 16.54 & 17.58 & 1.04 & 0.06 & 0.33 & 34.77 & 37.12 & -23.9 & 31.9 & 1.89 & 0.314 & E(APS), \\
\hline
\end{tabular}


White, Becker, et al.

TABLE 2-Continued

\begin{tabular}{|c|c|c|c|c|c|c|c|c|c|c|c|c|c|c|}
\hline $\begin{array}{l}\text { RA } \\
(1)\end{array}$ & $\begin{array}{l}\text { Dec } \\
(2)\end{array}$ & $\begin{array}{l}\mathrm{Cl} \\
(3)\end{array}$ & $\begin{array}{c}\mathrm{E} \\
(4)\end{array}$ & $\begin{array}{c}\mathrm{O} \\
(5)\end{array}$ & $\begin{array}{c}\text { O-E } \\
(6)\end{array}$ & $\begin{array}{c}\mathrm{A}(\mathrm{E}) \\
(7)\end{array}$ & $\begin{array}{c}\text { Sep } \\
(8)\end{array}$ & $\begin{array}{l}S_{p} \\
(9)\end{array}$ & $\begin{array}{c}S_{i} \\
(10)\end{array}$ & $\begin{array}{c}M_{B} \\
(11)\end{array}$ & $\begin{array}{c}\log L_{R} \\
\text { (12) }\end{array}$ & $\begin{array}{c}\log R^{*} \\
\text { (13) }\end{array}$ & $\begin{array}{c}\mathrm{Z} \\
(14)\end{array}$ & $\begin{array}{c}\text { Notes } \\
(15)\end{array}$ \\
\hline 114759.763 & +263542.47 & $*$ & 16.92 & 17.43 & 0.52 & 0.06 & 0.31 & 294.50 & 303.72 & -26.5 & 33.7 & 2.66 & 0.870 & o,X,No spec \\
\hline 114818.883 & +315409.81 & $*$ & 16.67 & 16.98 & 0.31 & 0.05 & 0.30 & 48.24 & 51.30 & -25.8 & 32.5 & 1.75 & 0.549 & o,X,No spec \\
\hline 114842.894 & +352052.57 & $*$ & 16.52 & 17.53 & 1.01 & 0.05 & 0.29 & 2.65 & 3.21 & -27.2 & 32.0 & 0.69 & 1.218 & \\
\hline 114851.474 & +231340.58 & $*$ & 17.46 & 19.16 & 1.70 & 0.05 & 0.18 & 5.73 & 5.63 & -27.8 & 33.0 & 1.46 & 3.077 & \\
\hline 114921.604 & +333857.85 & $*$ & 17.77 & 18.71 & 0.94 & 0.05 & 0.34 & 6.62 & 7.20 & -26.6 & 32.6 & 1.48 & 1.560 & \\
\hline 115019.580 & +334143.89 & $*$ & 17.45 & 17.91 & 0.47 & 0.06 & 0.32 & 98.64 & 229.85 & -27.3 & 34.0 & 2.67 & 1.490 & o,No spec \\
\hline 115023.570 & +281907.50 & $*$ & 16.46 & 18.00 & 1.54 & 0.06 & 0.10 & 13.96 & 14.22 & -29.0 & 33.4 & 1.39 & 3.124 & HiBAL?,dmpL $\alpha$ \\
\hline 115034.776 & +415440.11 & $*$ & 16.41 & 17.05 & 0.64 & 0.05 & 0.14 & 21.60 & 22.76 & -27.2 & 32.7 & 1.37 & 1.018 & $\mathrm{X}$ \\
\hline 115102.122 & +314643.31 & $*$ & 16.89 & 17.18 & 0.29 & 0.06 & 0.39 & 6.16 & 6.57 & -26.3 & 31.9 & 0.92 & 0.717 & \\
\hline 115117.757 & +382221.75 & $*$ & 15.02 & 15.50 & 0.48 & 0.06 & 0.25 & 10.93 & 10.79 & -26.2 & 31.4 & 0.52 & 0.336 & \\
\hline 115129.399 & +382552.57 & $*$ & 16.49 & 16.87 & 0.38 & 0.06 & 0.38 & 391.60 & 656.16 & -28.0 & 34.4 & 2.73 & 1.304 & o \\
\hline 115139.677 & +335541.74 & $*$ & 17.39 & 18.07 & 0.68 & 0.05 & 0.62 & 6.65 & 6.58 & -25.8 & 32.0 & 1.26 & 0.850 & \\
\hline 115227.514 & +320959.27 & $*$ & 16.93 & 17.30 & 0.37 & 0.06 & 0.52 & 29.02 & 32.92 & -24.6 & 32.0 & 1.71 & 0.374 & o,X,No spec \\
\hline 115251.909 & +3307 18.96 & $*$ & 15.75 & 16.18 & 0.43 & 0.05 & 0.53 & 187.17 & 190.31 & -28.8 & 33.9 & 1.91 & 1.389 & \\
\hline 115258.777 & +293014.86 & $*$ & 17.00 & 18.19 & 1.20 & 0.05 & 0.19 & 122.66 & 130.32 & -26.5 & 33.6 & 2.56 & 1.227 & P1 \\
\hline 115326.704 & +361726.26 & $*$ & 16.50 & 17.12 & 0.62 & 0.06 & 0.40 & 61.74 & 63.20 & -27.8 & 33.4 & 1.81 & 1.336 & $\mathrm{X}$ \\
\hline 115336.385 & +422636.30 & $*$ & 17.50 & 18.21 & 0.70 & 0.04 & 0.58 & 1.40 & 1.07 & -27.1 & 31.8 & 0.57 & 1.530 & \\
\hline 115344.729 & +403904.90 & $*$ & 17.38 & 17.79 & 0.40 & 0.04 & 0.24 & 6.75 & 6.75 & -27.1 & 32.4 & 1.10 & 1.317 & \\
\hline 115446.214 & +290231.78 & $*$ & 17.66 & 19.26 & 1.60 & 0.06 & 0.45 & 16.71 & 18.14 & -22.5 & 31.7 & 2.24 & 0.349 & \\
\hline 115607.848 & +373414.75 & $*$ & 17.12 & 17.81 & 0.70 & 0.05 & 0.20 & 4.10 & 3.98 & -28.6 & 32.7 & 0.81 & 2.456 & \\
\hline 115757.736 & +261325.24 & $*$ & 17.03 & 18.17 & 1.14 & 0.06 & 0.05 & 1.53 & 1.41 & -23.4 & 30.6 & 0.73 & 0.324 & \\
\hline 115917.315 & +283814.85 & $*$ & 17.37 & 18.19 & 0.82 & 0.06 & 0.44 & 2.22 & 2.01 & -22.4 & 30.3 & 0.92 & 0.210 & \\
\hline 115931.842 & +291443.94 & $*$ & 16.05 & 17.00 & 0.94 & 0.05 & 0.38 & 1855.80 & 1952.59 & -26.5 & 34.4 & 3.32 & 0.724 & $0, X$ \\
\hline 120051.501 & +350831.41 & $\mathrm{gE}$ & 15.21 & 16.45 & 1.24 & 0.05 & 0.69 & 2.03 & 1.46 & -29.1 & 32.1 & 0.01 & 1.700 & o,HiBAL \\
\hline 120240.678 & +263138.59 & $*$ & 17.23 & 17.35 & 0.12 & 0.06 & 0.59 & 61.67 & 63.84 & -25.1 & 32.5 & 2.00 & 0.478 & $\mathrm{X}$ \\
\hline 120243.556 & +373551.69 & $*$ & 17.31 & 17.46 & 0.15 & 0.04 & 0.38 & 16.29 & 17.24 & -27.2 & 32.7 & 1.39 & 1.194 & $z$ \\
\hline 120437.585 & +285125.36 & $*$ & 16.26 & 17.16 & 0.90 & 0.05 & 0.49 & 6.24 & 5.57 & -27.4 & 32.2 & 0.84 & 1.129 & $\mathrm{P} 1$ \\
\hline 120646.814 & +414832.21 & $\mathrm{gE}$ & 17.52 & 18.44 & 0.92 & 0.04 & 1.04 & 6.32 & 6.59 & -27.2 & 32.7 & 1.32 & 1.806 & \\
\hline 120722.352 & +252728.24 & $*$ & 17.77 & 18.63 & 0.86 & 0.05 & 0.51 & 2.23 & 2.07 & -21.9 & 30.3 & 1.10 & 0.208 & \\
\hline 120811.320 & +411404.69 & $*$ & 17.61 & 18.14 & 0.53 & 0.04 & 0.22 & 1.22 & 1.37 & -27.4 & 31.9 & 0.52 & 1.736 & \\
\hline 120927.227 & +224259.14 & $*$ & 17.48 & 18.04 & 0.56 & 0.08 & 0.35 & 8.07 & 8.13 & -26.6 & 32.4 & 1.30 & 1.208 & \\
\hline 120945.239 & +321700.87 & $*$ & 16.65 & 16.99 & 0.34 & 0.05 & 0.85 & 1.66 & 1.95 & -22.8 & 30.0 & 0.40 & 0.145 & o,X,No spec \\
\hline 120949.894 & +223644.40 & $*$ & 17.73 & 18.37 & 0.64 & 0.08 & 0.33 & 1.95 & 2.13 & -27.2 & 32.1 & 0.80 & 1.722 & \\
\hline 121037.577 & +315706.03 & $*$ & 16.34 & 16.57 & 0.23 & 0.05 & 0.10 & 19.15 & 22.04 & -25.4 & 31.9 & 1.24 & 0.388 & $\mathrm{o}, \mathrm{X}$ \\
\hline 121404.115 & +330945.74 & $*$ & 16.59 & 16.97 & 0.39 & 0.04 & 0.20 & 1372.84 & 1417.99 & -28.4 & 34.9 & 3.08 & 1.600 & o,No spec \\
\hline 121442.303 & +280329.01 & $*$ & 15.87 & 17.03 & 1.16 & 0.06 & 0.14 & 2.61 & 2.90 & -26.3 & 31.5 & 0.50 & 0.698 & FeLoBAL \\
\hline 121512.000 & +383832.09 & $\mathrm{gO}$ & 17.55 & 17.08 & -0.47 & 0.04 & 0.27 & 2.15 & 1.78 & -25.6 & 31.1 & 0.42 & 0.516 & \\
\hline 121721.415 & +305630.65 & $\mathrm{gE}$ & 16.76 & 17.37 & 0.62 & 0.04 & 0.11 & 8.69 & 8.67 & -24.0 & 31.2 & 1.18 & 0.300 & $o, X$ \\
\hline 121732.545 & +330538.22 & $*$ & 17.51 & 17.76 & 0.25 & 0.04 & 0.27 & 183.25 & 188.85 & -28.8 & 34.4 & 2.45 & 2.605 & o,No spec \\
\hline 122035.131 & +3853 17.12 & $*$ & 16.32 & 16.49 & 0.17 & 0.07 & 0.37 & 2.02 & 2.23 & -25.4 & 30.9 & 0.22 & 0.376 & \\
\hline 122313.387 & +290824.05 & $*$ & 16.83 & 17.51 & 0.68 & 0.06 & 0.15 & 1.46 & 0.82 & -27.0 & 31.6 & 0.35 & 1.126 & P1 \\
\hline 122424.235 & +401510.72 & $\mathrm{gE}$ & 17.54 & 17.79 & 0.26 & 0.06 & 0.24 & 39.84 & 41.56 & -24.4 & 32.2 & 2.00 & 0.415 & \\
\hline 122516.970 & +314535.01 & $*$ & 17.19 & 17.65 & 0.45 & 0.06 & 0.18 & 4.35 & 7.59 & -27.2 & 32.4 & 1.10 & 1.274 & \\
\hline 122527.389 & +223512.72 & $*$ & 16.16 & 16.32 & 0.16 & 0.06 & 0.52 & 3.86 & 5.69 & -29.6 & 32.7 & 0.39 & 2.043 & $\mathrm{o}$ \\
\hline 122539.555 & +245836.36 & $\mathrm{gE}$ & 15.96 & 16.65 & 0.69 & 0.05 & 0.28 & 6.87 & 8.32 & -24.5 & 31.1 & 0.87 & 0.268 & $0, X$ \\
\hline 122624.215 & +324429.37 & $\mathrm{gE}$ & 16.35 & 17.01 & 0.66 & 0.05 & 0.44 & 1.49 & 2.81 & -23.9 & 30.6 & 0.55 & 0.242 & $X$ \\
\hline 122749.151 & +321459.04 & $*$ & 16.82 & 18.75 & 1.93 & 0.05 & 0.24 & 6.54 & 6.42 & -20.9 & 30.4 & 1.63 & 0.137 & $\mathrm{X}$ \\
\hline 122824.988 & +312837.74 & $*$ & 15.51 & 15.88 & 0.37 & 0.04 & 0.35 & 315.34 & 323.78 & -30.3 & 34.5 & 1.95 & 2.199 & o \\
\hline 123001.002 & +290357.29 & $*$ & 16.41 & 16.82 & 0.42 & 0.07 & 0.43 & 2.42 & 1.99 & -28.5 & 32.1 & 0.25 & 1.569 & $\mathrm{P} 1$ \\
\hline 123010.336 & +283040.85 & $*$ & 17.26 & 17.99 & 0.73 & 0.05 & 0.61 & 1.18 & 1.89 & -26.7 & 31.8 & 0.64 & 1.226 & \\
\hline 123143.589 & +284749.71 & $\mathrm{gE}$ & 15.93 & 16.70 & 0.77 & 0.06 & 0.30 & 93.78 & 97.85 & -27.5 & 33.3 & 1.86 & 1.000 & 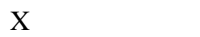 \\
\hline 123155.985 & +353015.12 & $\mathrm{gO}$ & 14.87 & 15.96 & 1.09 & 0.04 & 0.14 & 3.50 & 3.68 & -23.6 & 30.2 & 0.27 & 0.131 & X,I \\
\hline 123252.693 & +414321.86 & $*$ & 17.58 & 18.10 & 0.52 & 0.05 & 0.47 & 2.66 & 2.54 & -25.6 & 31.6 & 0.88 & 0.794 & \\
\hline 123252.745 & +301523.97 & $*$ & 17.40 & 18.05 & 0.66 & 0.05 & 0.39 & 3.70 & 3.72 & -25.9 & 31.8 & 1.00 & 0.898 & P1 \\
\hline 123338.687 & +422239.12 & $*$ & 17.45 & 18.69 & 1.24 & 0.06 & 0.84 & 1.14 & 2.65 & -25.5 & 31.8 & 1.09 & 0.982 & \\
\hline 123355.515 & +290748.94 & $*$ & 16.21 & 16.84 & 0.63 & 0.05 & 0.15 & 1.09 & 0.74 & -29.1 & 32.0 & -0.12 & 2.013 & o \\
\hline 123425.658 & +243143.92 & $*$ & 17.27 & 17.77 & 0.50 & 0.05 & 0.21 & 510.65 & 547.28 & -27.2 & 34.3 & 3.01 & 1.340 & o \\
\hline 123651.570 & +250750.67 & $\mathrm{gE}$ & 17.27 & 17.60 & 0.33 & 0.04 & 0.46 & 16.25 & 16.97 & -25.2 & 32.1 & 1.52 & 0.550 & o,X,No spec \\
\hline 123812.215 & +271256.21 & $\mathrm{gO}$ & 17.65 & 19.25 & 1.59 & 0.04 & 0.05 & 1.12 & 1.15 & -21.6 & 30.2 & 1.06 & 0.235 & \\
\hline 123915.040 & +404956.07 & $*$ & 17.80 & 18.65 & 0.85 & 0.05 & 0.94 & 56.64 & 65.61 & -26.2 & 33.4 & 2.44 & 1.318 & \\
\hline 124009.142 & +242531.07 & $*$ & 16.35 & 16.83 & 0.48 & 0.04 & 0.27 & 30.16 & 32.36 & -27.0 & 32.7 & 1.45 & 0.829 & $X$ \\
\hline 124503.587 & +422844.92 & $\mathrm{gE}$ & 17.56 & 18.16 & 0.60 & 0.06 & 0.85 & 1.44 & 2.18 & -24.3 & 31.0 & 0.86 & 0.478 & \\
\hline 124511.268 & +335610.23 & $*$ & 16.60 & 17.15 & 0.55 & 0.04 & 0.31 & 1.88 & 1.23 & -26.3 & 31.3 & 0.36 & 0.709 & o \\
\hline 124720.821 & +320900.89 & $*$ & 17.14 & 17.97 & 0.82 & 0.04 & 0.29 & 77.07 & 132.09 & -26.1 & 33.4 & 2.51 & 0.947 & $\mathrm{o}, \mathrm{X}$ \\
\hline 124813.883 & +362423.96 & $\mathrm{gE}$ & 16.75 & 18.61 & 1.86 & 0.04 & 0.70 & 2.36 & 2.93 & -22.0 & 30.4 & 1.21 & 0.206 & $\mathrm{X}$ \\
\hline 124918.397 & +281743.85 & $*$ & 17.71 & 18.36 & 0.65 & 0.04 & 0.12 & 70.33 & 73.42 & -27.2 & 33.7 & 2.34 & 1.705 & \\
\hline 125224.970 & +291321.17 & $*$ & 16.97 & 17.37 & 0.41 & 0.04 & 0.45 & 1.37 & 0.76 & -26.4 & 31.3 & 0.30 & 0.824 & $\mathrm{o}$ \\
\hline 125306.423 & +290513.85 & $*$ & 17.49 & 17.72 & 0.23 & 0.03 & 0.25 & 60.91 & 61.78 & -28.8 & 33.9 & 1.95 & 2.573 & P1 \\
\hline 125317.583 & +310550.41 & $*$ & 16.42 & 16.71 & 0.29 & 0.04 & 0.70 & 1.76 & 1.60 & -26.9 & 31.4 & 0.15 & 0.782 & $\mathrm{o}, \mathrm{I}$ \\
\hline 125602.065 & +385230.74 & $*$ & 16.97 & 17.89 & 0.92 & 0.05 & 0.19 & 2.05 & 2.10 & -24.3 & 30.9 & 0.75 & 0.419 & \\
\hline 125718.306 & +245442.67 & $*$ & 17.39 & 17.75 & 0.36 & 0.03 & 0.33 & 43.38 & 44.17 & -27.4 & 33.3 & 1.89 & 1.487 & \\
\hline
\end{tabular}


TABLE 2-Continued

\begin{tabular}{|c|c|c|c|c|c|c|c|c|c|c|c|c|c|c|}
\hline $\begin{array}{l}\text { RA } \\
\text { (1) }\end{array}$ & $\begin{array}{c}\text { Dec } \\
(2)\end{array}$ & $\begin{array}{l}\mathrm{Cl} \\
(3)\end{array}$ & $\begin{array}{c}E \\
(4)\end{array}$ & $\begin{array}{c}\mathrm{O} \\
(5)\end{array}$ & $\begin{array}{c}\text { O-E } \\
(6)\end{array}$ & $\begin{array}{c}\mathrm{A}(\mathrm{E}) \\
(7)\end{array}$ & $\begin{array}{c}\text { Sep } \\
(8)\end{array}$ & $\begin{array}{l}S_{p} \\
(9)\end{array}$ & $\begin{array}{c}S_{i} \\
(10)\end{array}$ & $\begin{array}{c}M_{B} \\
(11)\end{array}$ & $\begin{array}{c}\log L_{R} \\
\text { (12) }\end{array}$ & $\begin{array}{c}\log R^{*} \\
\text { (13) }\end{array}$ & $\begin{array}{c}\mathrm{Z} \\
(14)\end{array}$ & $\begin{array}{c}\text { Notes } \\
(15)\end{array}$ \\
\hline 125723.863 & 364419.39 & $\mathrm{gE}$ & 7.64 & 18.40 & .76 & 0.04 & 0.69 & 690.34 & 716.97 & -25.0 & 33.9 & 3.44 & 0.710 & $\mathrm{o}, \mathrm{Nc}$ \\
\hline 125731.348 & +404206.74 & $*$ & 17.58 & 18.49 & 0.91 & 0.04 & 1.03 & 10.80 & 14.08 & -26.3 & 32.7 & 1.71 & 1.260 & \\
\hline 125807.457 & +232921.87 & $*$ & 16.69 & 17.47 & 0.78 & 0.05 & 0.49 & 4.16 & 3.71 & -27.2 & 32.1 & 0.78 & 1.185 & \\
\hline 125829.695 & +352843.27 & $\mathrm{gO}$ & 17.40 & 18.42 & 1.02 & 0.03 & 0.51 & 15.55 & 21.48 & -27.3 & 33.2 & 1.81 & 1.880 & $\mathrm{o}, \mathrm{No}$ \\
\hline 125948.789 & +342322.64 & $*$ & 16.10 & 16.91 & 0.81 & 0.03 & 0.08 & 10.56 & 12.25 & -28.1 & 32.7 & 1.01 & 1.377 & $\mathrm{o}, \mathrm{X}$ \\
\hline 130028.544 & +283010.20 & $\mathrm{gO}$ & 17.07 & 17.74 & 0.66 & 0.03 & 0.41 & 192.10 & 195.64 & -25.5 & 33.3 & 2.62 & 0.646 & o \\
\hline 130203.082 & +265809.09 & $*$ & 17.56 & 18.00 & 0.44 & 0.03 & 0.16 & 7.38 & 7.31 & -26.2 & 32.2 & 1.26 & 0.992 & \\
\hline 130229.340 & +413222.59 & $*$ & 17.59 & 18.38 & 0.79 & 0.04 & 0.22 & 17.22 & 20.99 & -26.5 & 32.9 & 1.84 & 1.290 & \\
\hline 130425.543 & +421009.66 & $*$ & 16.33 & 17.08 & 0.75 & 0.04 & 0.35 & 1.52 & 0.99 & -28.7 & 32.1 & 0.13 & 1.916 & o,HiBAL \\
\hline 130433.441 & +320635.79 & $*$ & 17.07 & 18.15 & 1.08 & 0.04 & 0.49 & 10.48 & 10.15 & -26.8 & 32.6 & 1.44 & 1.333 & \\
\hline 130451.426 & +245445.96 & $*$ & 17.12 & 17.51 & 0.38 & 0.04 & 0.26 & 22.21 & 23.56 & -25.5 & 32.3 & 1.62 & 0.604 & $\mathrm{X}$ \\
\hline 130613.330 & +391526.60 & $*$ & 15.71 & 16.06 & 0.35 & 0.04 & 0.49 & 4.45 & 3.77 & -26.3 & 31.3 & 0.34 & 0.447 & \\
\hline 130621.808 & +313359.84 & $\mathrm{gE}$ & 17.59 & 18.33 & 0.75 & 0.03 & 0.55 & 4.46 & 4.52 & -23.7 & 31.2 & 1.26 & 0.396 & \\
\hline 130641.679 & +414827.51 & $*$ & 17.23 & 18.07 & 0.84 & 0.05 & 0.13 & 32.19 & 33.45 & -26.5 & 33.0 & 1.93 & 1.157 & \\
\hline 130744.354 & +360922.21 & $*$ & 17.24 & 18.01 & 0.77 & 0.04 & 1.02 & 1.20 & 1.10 & -27.0 & 31.7 & 0.44 & 1.402 & $z$ \\
\hline 130944.933 & +222632.00 & $*$ & 17.24 & 17.87 & 0.64 & 0.04 & 0.55 & 1.72 & 1.81 & -28.0 & 32.2 & 0.52 & 1.957 & \\
\hline 130952.389 & +274240.85 & $*$ & 17.64 & 18.05 & 0.41 & 0.03 & 0.61 & 1.99 & 2.13 & -25.3 & 31.4 & 0.78 & 0.699 & \\
\hline 131016.748 & +334419.60 & $*$ & 17.73 & 18.12 & 0.38 & 0.03 & 0.01 & 17.23 & 17.77 & -27.5 & 33.1 & 1.62 & 1.780 & $\mathrm{o}, \mathrm{Nc}$ \\
\hline 131059.404 & +323334.59 & $*$ & 16.47 & 17.08 & 0.61 & 0.03 & 0.17 & 243.42 & 245.97 & -28.4 & 34.2 & 2.36 & 1.650 & \\
\hline 131132.065 & +373408.01 & $\mathrm{gO}$ & 17.20 & 17.41 & 0.20 & 0.04 & 0.78 & 1.20 & 1.53 & -27.4 & 31.7 & 0.31 & 1.272 & o \\
\hline 131213.560 & +231958.51 & $*$ & 16.95 & 17.84 & 0.89 & 0.03 & 0.37 & 43.27 & 44.12 & -27.4 & 33.3 & 1.92 & 1.508 & HiBAL \\
\hline 131217.763 & +351521.25 & $\mathrm{gE}$ & 15.17 & 15.86 & 0.70 & 0.03 & 0.23 & 43.92 & 44.72 & -24.4 & 31.5 & 1.30 & 0.183 & $\mathrm{o}, \mathrm{X}$ \\
\hline 131314.271 & +375357.21 & $*$ & 16.73 & 17.10 & 0.38 & 0.04 & 0.25 & 2.48 & 2.44 & -26.1 & 31.4 & 0.47 & 0.656 & \\
\hline 131423.149 & +291001.86 & $*$ & 17.49 & 18.26 & 0.77 & 0.03 & 0.18 & 7.53 & 7.22 & -26.2 & 32.3 & 1.36 & 1.094 & P1 \\
\hline 131435.185 & +301802.90 & $*$ & 17.65 & 18.46 & 0.81 & 0.03 & 0.33 & 7.07 & 7.30 & -27.4 & 32.8 & 1.36 & 1.920 & \\
\hline 131718.653 & +392528.02 & $*$ & 17.80 & 18.59 & 0.79 & 0.04 & 0.21 & 473.47 & 614.98 & -26.7 & 34.5 & 3.36 & 1.560 & $\mathrm{o}, \mathrm{N}$ \\
\hline 132033.219 & +331142.35 & $*$ & 17.52 & 17.98 & 0.46 & 0.04 & 0.26 & 22.35 & 23.51 & -26.1 & 32.6 & 1.76 & 0.924 & \\
\hline 132106.686 & +374153.41 & $*$ & 17.68 & 17.73 & 0.05 & 0.03 & 0.46 & 8.73 & 8.43 & -26.8 & 32.4 & 1.21 & 1.135 & \\
\hline 132146.519 & +265149.99 & $*$ & 16.57 & 17.02 & 0.45 & 0.06 & 0.79 & 2.62 & 1.81 & -26.8 & 31.6 & 0.44 & 0.844 & \\
\hline 132255.672 & +391207.91 & $*$ & 17.23 & 18.24 & 1.01 & 0.03 & 0.65 & 101.01 & 102.01 & -28.6 & 34.3 & 2.35 & 2.982 & o \\
\hline 132308.223 & +230958.04 & $\mathrm{gE}$ & 15.99 & 17.97 & 1.99 & 0.05 & 0.73 & 1.54 & 2.73 & -21.8 & 30.1 & 0.94 & 0.145 & \\
\hline 132355.675 & +250834.31 & $*$ & 17.49 & 18.77 & 1.27 & 0.04 & 0.14 & 99.39 & 100.53 & -26.5 & 33.7 & 2.65 & 1.528 & \\
\hline 132422.536 & +245222.25 & $\mathrm{gE}$ & 17.40 & 18.87 & 1.47 & 0.04 & 0.63 & & 4.41 & -27.4 & 32.8 & 1.32 & 2.357 & \\
\hline 132515.008 & +330556.19 & $*$ & 16.94 & 17.61 & 0.67 & 0.03 & 0.24 & 1.31 & 0.84 & -24.2 & 30.6 & 0.44 & 0.356 & \\
\hline 132550.783 & +355334.58 & $*$ & 17.35 & 17.89 & 0.54 & 0.03 & 0.86 & 1.55 & 0.89 & -27.1 & 31.8 & 0.50 & 1.358 & \\
\hline 132629.102 & +362334.91 & $*$ & 17.04 & 17.76 & 0.72 & 0.03 & 0.28 & & 1.07 & -24.2 & 30.8 & 0.62 & 0.389 & \\
\hline 132735.577 & +240742.68 & $*$ & 17.06 & 18.17 & 1.11 & 0.04 & 0.33 & 46.87 & 46.94 & -26.5 & 33.2 & 2.11 & 1.201 & \\
\hline 132816.089 & +230916.50 & $*$ & 17.67 & 18.56 & 0.88 & 0.06 & 0.19 & 1.40 & 1.24 & -23.4 & 30.7 & 0.84 & 0.387 & \\
\hline 13290 & & $*$ & 07 & 17.92 & 0.85 & 0.03 & 0.99 & & 22.46 & 3 & 2.7 & 1.71 & 0.990 & o,No \\
\hline 132909.161 & +221349.55 & $*$ & 17.48 & 18.52 & 1.03 & 0.05 & 0.09 & 6.48 & 6.19 & -25.8 & 32.2 & 1.41 & 1.051 & \\
\hline 133037.694 & +250910.87 & $\mathrm{gE}$ & 16.52 & 18.23 & 1.72 & 0.03 & 0.39 & 6826.39 & 6999.01 & -26.1 & 35.2 & 4.33 & 1.055 & o \\
\hline 13310 & +303032.95 & $*$ & 16.89 & 17.23 & 0.34 & 0.03 & 0.27 & 147 & 15023.95 & -26.6 & .4 & 4.28 & 0.848 & o \\
\hline 133126.340 & +375604.97 & $*$ & 17.32 & 18.17 & 0.84 & 0.02 & 0.74 & 6.61 & 6.80 & -26.2 & 32.2 & 1.29 & 1.048 & \\
\hline 133309.753 & +362030.86 & $*$ & 17.21 & 17.61 & 0.40 & 0.03 & 0.03 & 16.63 & 17.50 & -25.7 & 32.3 & 1.52 & 0.679 & \\
\hline 133317.602 & +30491 & $*$ & 17.35 & 18.03 & 0.67 & 0.04 & 0.24 & 15.26 & 16.09 & -27.4 & 33.0 & 1.55 & 1.639 & \\
\hline 133345.466 & +414128.17 & $*$ & 17.20 & 18.30 & 1.10 & 0.02 & 0.46 & 2.47 & 1.95 & -22.5 & 30.5 & 1.01 & 0.225 & $\mathrm{X}$ \\
\hline 133403.842 & +370104.00 & $*$ & 16.84 & 17.84 & 1.00 & 0.02 & 0.51 & 13.17 & 13.31 & -27.8 & 32.9 & 1.38 & 1.765 & \\
\hline 133449.725 & +312824.09 & $*$ & 17.26 & 17.78 & 0.52 & 0.03 & 0.35 & 21.27 & 22.39 & -27.1 & 32.9 & 1.63 & 1.311 & \\
\hline 133739.872 & +390916.38 & $*$ & 8.10 & 9.17 & 1.07 & 0.02 & 0.67 & 1.68 & 2.47 & -26.2 & 28.4 & -2.60 & 0.020 & $\mathrm{o}, \mathrm{I}, \mathrm{B}=14.2$ \\
\hline 134005.764 & +343707.86 & $\mathrm{gO}$ & 17.14 & 18.76 & 1.62 & 0.03 & 0.41 & 1.33 & 1.61 & -21.9 & 30.2 & 1.01 & 0.218 & \\
\hline 134009.122 & +235517.93 & $*$ & 16.99 & 17.57 & 0.58 & 0.04 & 0.14 & 17.89 & 18.99 & -27.3 & 32.9 & 1.47 & 1.327 & \\
\hline 134133.117 & +353251.94 & $*$ & 17.62 & 18.17 & 0.54 & 0.03 & 0.38 & 52.49 & 57.42 & -25.5 & 32.9 & 2.24 & 0.780 & \\
\hline 134159.915 & +3707 10.20 & $*$ & 16.32 & 16.73 & 0.41 & 0.02 & 0.55 & 24.50 & 25.42 & -27.7 & 32.8 & 1.28 & 1.106 & $\Lambda$ \\
\hline 134254.390 & +282805.92 & $*$ & 17.57 & 18.09 & 0.53 & 0.04 & 0.37 & 65.57 & 68.41 & -26.2 & 33.2 & 2.26 & 1.040 & o,No spec \\
\hline 134300.121 & +284407.02 & $*$ & 16.18 & 16.81 & 0.63 & 0.04 & 1.01 & 217.37 & 246.65 & -27.2 & 33.7 & 2.32 & 0.905 & $\mathrm{o}, \mathrm{X}$ \\
\hline 134454.472 & +421338.68 & $*$ & 17.46 & 18.14 & 0.69 & 0.02 & 0.35 & 11.51 & 11.00 & -27.0 & 32.7 & 1.47 & 1.468 & \\
\hline 134520.421 & +324112.59 & $*$ & 17.55 & 17.86 & 0.31 & 0.04 & 0.28 & 13.90 & 14.62 & -28.3 & 33.2 & 1.40 & 2.253 & \\
\hline 134525.703 & +371219.48 & $*$ & 17.39 & 17.83 & 0.44 & 0.02 & 0.74 & 1.19 & 0.67 & -26.3 & 31.4 & 0.41 & 0.962 & \\
\hline 134536.948 & +382312.62 & $*$ & 17.67 & 18.36 & 0.69 & 0.03 & 0.40 & 845.79 & 853.81 & -27.4 & 34.8 & 3.39 & 1.844 & O(APS),o \\
\hline 134609.765 & +254059.84 & $*$ & 17.74 & 18.75 & 1.01 & 0.03 & 0.14 & 17.20 & 18.70 & -23.3 & 31.8 & 2.04 & 0.390 & \\
\hline 134635.072 & +312133.84 & $*$ & 17.70 & 18.52 & 0.82 & 0.04 & 1.14 & 1.24 & 1.37 & -22.5 & 30.3 & 0.84 & 0.247 & \\
\hline 134645.498 & +231137.54 & $*$ & 17.54 & 19.17 & 1.63 & 0.04 & 0.29 & 1.11 & 1.99 & -23.0 & 30.9 & 1.24 & 0.410 & \\
\hline 134737.449 & +301252.12 & $\mathrm{gE}$ & 15.78 & 16.23 & 0.45 & 0.05 & 0.18 & 15.42 & 15.88 & -23.1 & 30.7 & 1.01 & 0.118 & $\mathrm{P} 1$ \\
\hline 134804.313 & +284025.43 & $*$ & 17.51 & 17.67 & 0.16 & 0.04 & 0.43 & 72.45 & 78.07 & -28.8 & 34.0 & 2.03 & 2.464 & $\mathrm{P} 1$ \\
\hline 134820.885 & +302005.59 & $*$ & 17.49 & 17.83 & 0.34 & 0.05 & 0.08 & 22.49 & 23.53 & -27.9 & 33.2 & 1.62 & 1.865 & $\mathrm{P} 1$ \\
\hline 135015.151 & +381206.77 & $*$ & 17.51 & 17.30 & -0.20 & 0.04 & 0.86 & 77.34 & 123.44 & -27.7 & 33.7 & 2.17 & 1.390 & o,No spec \\
\hline 135326.133 & +362049.24 & $\mathrm{gE}$ & 14.70 & 15.41 & 0.71 & 0.04 & 0.59 & 1.70 & 1.57 & -25.9 & 30.5 & -0.32 & 0.286 & o \\
\hline 135335.925 & +263147.54 & $*$ & 17.20 & 17.72 & 0.52 & 0.04 & 0.34 & 22.35 & 23.63 & -23.7 & 31.7 & 1.75 & 0.307 & $\mathrm{o}, \mathrm{X}$ \\
\hline 135430.686 & +331346.98 & $*$ & 17.63 & 18.06 & 0.42 & 0.05 & 0.51 & 16.36 & 16.38 & -25.6 & 32.4 & 1.65 & 0.795 & \\
\hline 135529.883 & +293059.27 & $*$ & 17.26 & 18.16 & 0.90 & 0.04 & 0.46 & 1.74 & 1.54 & -23.3 & 30.6 & 0.79 & 0.302 & PI \\
\hline 135607.365 & +413615.34 & $*$ & 16.23 & 16.71 & 0.48 & 0.01 & 0.20 & 23.47 & 24.31 & -26.6 & 32.4 & 1.30 & 0.691 & $\mathrm{o}, \mathrm{X}$ \\
\hline
\end{tabular}


White, Becker, et al.

TABLE 2-Continued

\begin{tabular}{|c|c|c|c|c|c|c|c|c|c|c|c|c|c|c|}
\hline $\begin{array}{l}\text { RA } \\
\text { (1) }\end{array}$ & $\begin{array}{l}\text { Dec } \\
(2)\end{array}$ & $\begin{array}{l}\mathrm{Cl} \\
(3)\end{array}$ & $\begin{array}{c}E \\
(4)\end{array}$ & $\begin{array}{c}\mathrm{O} \\
(5)\end{array}$ & $\begin{array}{c}\text { O-E } \\
(6)\end{array}$ & $\begin{array}{c}\mathrm{A}(\mathrm{E}) \\
(7)\end{array}$ & $\begin{array}{c}\text { Sep } \\
(8)\end{array}$ & $\begin{array}{l}S_{p} \\
(9)\end{array}$ & $\begin{array}{c}S_{i} \\
(10)\end{array}$ & $\begin{array}{l}M_{B} \\
(11)\end{array}$ & $\begin{array}{c}\log L_{R} \\
\text { (12) }\end{array}$ & $\begin{array}{c}\log R^{*} \\
\text { (13) }\end{array}$ & $\begin{array}{c}\mathrm{Z} \\
(14)\end{array}$ & $\begin{array}{l}\text { Notes } \\
(15)\end{array}$ \\
\hline 135640.380 & 243100.36 & $*$ & 17.26 & 7.98 & .72 & 0.04 & 0 & 44.20 & 45.04 & -26.4 & 33.0 & 2.03 & 1.044 & \\
\hline 135845.399 & +265808.34 & $*$ & 17.69 & 18.76 & 1.07 & 0.06 & 0.38 & 1.82 & 1.17 & -22.9 & 30.7 & 1.05 & 0.330 & \\
\hline 135954.844 & +225223.92 & $*$ & 17.35 & 18.58 & 1.23 & 0.06 & 0.61 & 1.55 & 2.02 & -23.0 & 30.7 & 1.02 & 0.324 & \\
\hline 140012.603 & +353930.78 & $*$ & 17.06 & 17.57 & 0.52 & 0.03 & 0.31 & 2.15 & 2.10 & -25.1 & 31.1 & 0.61 & 0.525 & \\
\hline 140127.231 & +313812.90 & $\mathrm{gO}$ & 17.77 & 17.85 & 0.09 & 0.06 & 0.72 & 1.18 & 2.20 & -23.1 & 30.5 & 0.78 & 0.241 & \\
\hline 140146.991 & +232413.83 & $*$ & 16.08 & 16.78 & 0.71 & 0.07 & 0.83 & 1.04 & 1.50 & -23.9 & 30.2 & 0.19 & 0.213 & \\
\hline 140200.587 & +420442.18 & $\mathrm{gE}$ & 17.13 & 18.71 & 1.57 & 0.04 & 0.71 & 1.02 & 1.23 & -21.4 & 29.9 & 0.88 & 0.168 & \\
\hline 140504.815 & +265727.72 & $*$ & 16.98 & 17.26 & 0.28 & 0.05 & 0.35 & 5.21 & 4.91 & -26.2 & 31.8 & 0.85 & 0.713 & \\
\hline 140516.195 & +255534.93 & $\mathrm{gE}$ & 15.37 & 15.78 & 0.42 & 0.04 & 0.63 & 1.19 & 0.75 & -24.3 & 29.9 & -0.30 & 0.165 & $\mathrm{o}, \mathrm{X}, \mathrm{I}$ \\
\hline 140545.121 & +272515.31 & $*$ & 17.19 & 17.34 & 0.15 & 0.06 & 1.02 & 1.18 & 0.74 & -22.7 & 29.9 & 0.32 & 0.164 & $\mathrm{X}$ \\
\hline 140626.600 & +250921.30 & $\mathrm{gE}$ & 16.16 & 17.16 & 1.00 & 0.05 & 0.26 & 67.51 & 67.69 & -26.7 & 33.1 & 1.90 & 0.867 & o \\
\hline 140800.454 & +345125.11 & $*$ & 17.03 & 18.44 & 1.41 & 0.04 & 0.40 & 2.91 & 2.87 & -26.3 & 32.0 & 1.01 & 1.215 & LoBAL \\
\hline 140806.207 & +305448.67 & $*$ & 17.30 & 19.00 & 1.69 & 0.03 & 0.25 & 3.34 & 3.21 & -24.8 & 31.7 & 1.33 & 0.842 & LoBAL, $z$ ? \\
\hline 140827.822 & +240924.39 & $\mathrm{gE}$ & 15.26 & 16.58 & 1.32 & 0.05 & 0.12 & 3.59 & 3.02 & -23.0 & 30.1 & 0.50 & 0.131 & $\mathrm{X}$ \\
\hline 140923.904 & +261821.00 & $*$ & 15.38 & 15.64 & 0.27 & 0.04 & 0.16 & 8.85 & 9.19 & -28.5 & 32.3 & 0.42 & 0.945 & $\mathrm{o}, \mathrm{X}$ \\
\hline 141036.802 & +295551.08 & $\mathrm{gO}$ & 17.67 & 17.51 & -0.16 & 0.04 & 0.39 & 4.00 & 4.09 & -25.4 & 31.5 & 0.86 & 0.570 & $\mathrm{P} 1$ \\
\hline 141159.734 & +423950.43 & $*$ & 16.54 & 17.40 & 0.86 & 0.02 & 0.12 & 71.17 & 72.31 & -26.5 & 33.1 & 2.03 & 0.886 & $\mathrm{o}, \mathrm{X}$ \\
\hline 141334.404 & +421201.76 & $*$ & 17.60 & 18.40 & 0.80 & 0.02 & 0.14 & 17.79 & 18.74 & -28.3 & 33.5 & 1.69 & 2.810 & HiBAL \\
\hline 141528.473 & +3706 21.49 & $*$ & 17.43 & 18.33 & 0.90 & 0.02 & 0.36 & 406.30 & 409.69 & -28.0 & 34.7 & 3.03 & 2.360 & o \\
\hline 141617.381 & +264906.20 & $*$ & 17.69 & 18.44 & 0.76 & 0.04 & 0.54 & 2.94 & 2.60 & -27.8 & 32.5 & 0.93 & 2.297 & \\
\hline 141630.672 & +372137.09 & $*$ & 17.73 & 18.57 & 0.84 & 0.02 & 0.33 & 29.33 & 30.53 & -25.5 & 32.8 & 2.11 & 0.921 & $X$ \\
\hline 141717.882 & +231720.19 & $*$ & 17.51 & 17.93 & 0.42 & 0.06 & 0.21 & 43.26 & 48.27 & -26.1 & 33.0 & 2.06 & 0.921 & \\
\hline 141741.087 & +223528.77 & $*$ & 17.49 & 17.72 & 0.23 & 0.07 & 1.05 & 1.02 & 2.61 & -26.3 & 31.7 & 0.71 & 0.903 & \\
\hline 141842.235 & +423343.12 & $*$ & 15.98 & 16.37 & 0.39 & 0.03 & 0.24 & 1.73 & 1.25 & -25.8 & 30.8 & 0.05 & 0.421 & $\mathrm{o}, \mathrm{I}$ \\
\hline 141916.714 & +320303.04 & $\mathrm{gE}$ & 16.11 & 16.30 & 0.19 & 0.03 & 0.43 & 7.39 & 7.37 & -25.6 & 31.4 & 0.66 & 0.377 & \\
\hline 141917.364 & +310924.01 & $*$ & 17.06 & 18.28 & 1.23 & 0.04 & 0.57 & & 0.99 & -25.5 & 31.3 & 59 & 340 & \\
\hline 142013.072 & +253403.71 & $*$ & 16.78 & 18.24 & 1.47 & 0.05 & 0.57 & 1.27 & 1.17 & -27.9 & 32.1 & 0.49 & 2.200 & o,HiBAL \\
\hline 142106.049 & +385522.96 & $*$ & 17.73 & 18.74 & 1.02 & 0.03 & 0.21 & 94.32 & 97.91 & -23.8 & 32.7 & 2.75 & 0.490 & $\mathrm{O}(\mathrm{APS})$ \\
\hline 142108.724 & +224117.42 & $*$ & 16.76 & 16.87 & 0.11 & 0.11 & 0.03 & 4.73 & 5.21 & -29.3 & 32.7 & 0.56 & 2.181 & o \\
\hline 142114.075 & +282452.23 & $*$ & 17.10 & 17.51 & 0.41 & 0.04 & 0.46 & 46.79 & 48.74 & -25.3 & 32.5 & 1.94 & 0.538 & $\mathrm{P} 1$ \\
\hline 142230.375 & +322310.50 & $*$ & 17.14 & 17.47 & 0.32 & 0.03 & 0.83 & 412.83 & 425.66 & -25.9 & 33.6 & 2.85 & 0.685 & o,X,No spec \\
\hline 142255.380 & +325102.81 & $*$ & 7.25 & 8.38 & 1.13 & 0.03 & 0.21 & 2.53 & 6.41 & -28.2 & 29.3 & -2.50 & 0.035 & $\mathrm{o}, \mathrm{I}, \mathrm{B}=1$ \\
\hline 142326.073 & +325220.34 & $*$ & 16.34 & 16.58 & 0.24 & 0.03 & 0.42 & 8.71 & 8.16 & -29.2 & 32.8 & 0.68 & 1.903 & o \\
\hline 142550.651 & +240403.53 & $*$ & 17.06 & 17.64 & 0.58 & 0.08 & 0.86 & 121.14 & 320.88 & -25.6 & 33.5 & 2.80 & 0.654 & $\mathrm{o}, \mathrm{X}$ \\
\hline 142606.195 & +402432.02 & $\mathrm{gE}$ & 17.05 & 17.78 & 0.73 & 0.03 & 0.11 & 26.26 & 26.66 & -25.5 & 32.4 & 1.77 & 0.665 & $0, X$ \\
\hline 142634.445 & +224504.19 & $*$ & 17.03 & 17.49 & 0.46 & 0.15 & 0.50 & 1.03 & 0.77 & -26.8 & 31.4 & 0.20 & 1.033 & \\
\hline 142702.211 & +270122.51 & $*$ & 17.65 & 19.09 & 1.44 & 0.05 & 0.18 & 21.60 & 22.55 & -21.0 & 31.1 & 2.30 & 0.164 & \\
\hline 142703.637 & +270940.29 & $*$ & 17.68 & 19.05 & 1.37 & 0.05 & 0.30 & 2.58 & 2.98 & -25.6 & 32.0 & 1.27 & 1.170 & FeLoBAL \\
\hline 142735.609 & +263214.58 & $\mathrm{gO}$ & 15.79 & 15.79 & 0.00 & 0.05 & 0.48 & 42.72 & 44.67 & -26.1 & 32.1 & 1.24 & 0.368 & o \\
\hline 142807.909 & +223257.54 & $*$ & 17.64 & 18.37 & 0.72 & 0.15 & 0.40 & 1.79 & 1.94 & -26.5 & 31.9 & 0.80 & 1.319 & \\
\hline 142852.831 & +271042.53 & $*$ & 17.54 & 17.73 & 0.20 & 0.06 & 0.17 & 6.82 & 6.75 & -24.6 & 31.5 & 1.19 & 0.445 & \\
\hline 142950.889 & +260750.54 & $\mathrm{gE}$ & 17.13 & 18.20 & 1.07 & 0.05 & 0.57 & 359.79 & 382.67 & -28.6 & 34.8 & 2.91 & 2.910 & \\
\hline 143038.250 & +315917.49 & $*$ & 17.41 & 18.13 & 0.72 & 0.03 & 0.21 & 1.33 & 1.26 & -26.9 & 31.7 & 0.53 & 1.377 & \\
\hline 143120.533 & +395241.20 & $*$ & 15.66 & 16.63 & 0.97 & 0.03 & 0.42 & 207.87 & 210.04 & -28.1 & 33.8 & 2.15 & 1.217 & \\
\hline 143125.884 & +244220.53 & $*$ & 16.63 & 17.04 & 0.42 & 0.08 & 0.27 & 88.27 & 91.21 & -25.1 & 32.5 & 2.05 & 0.408 & \\
\hline 143144.905 & +391910.50 & $*$ & & & 1.31 & 0.03 & 0.08 & & 4.07 & -25.9 & 32.1 & 1.25 & 1.092 & \\
\hline 143157.947 & +341650.28 & $*$ & 16.43 & 16.76 & 0.33 & 0.04 & 0.11 & 1.22 & 0.65 & -26.6 & 31.1 & 0.02 & 0.704 & \\
\hline 143243.322 & +410328.04 & $*$ & 17.35 & 18.06 & 0.71 & 0.03 & 0.28 & 257.11 & 261.66 & -27.8 & 34.3 & 2.75 & 1.963 & \\
\hline 143401.123 & +405014.29 & $\mathrm{gE}$ & 17.07 & 18.19 & 1.12 & 0.04 & 0.25 & 1.94 & 1.83 & -27.0 & 32.0 & 0.71 & 1.483 & \\
\hline 143444.944 & +231743.01 & $\mathrm{gO}$ & 17.01 & 18.46 & 1.45 & 0.09 & 0.39 & 1.43 & 1.67 & -20.5 & 29.6 & 0.93 & 0.100 & $\mathrm{X}$ \\
\hline 143534.308 & +423408.91 & $*$ & 17.64 & 18.59 & 0.96 & 0.04 & 0.02 & 7.84 & 9.45 & -26.4 & 32.6 & 1.57 & 1.373 & \\
\hline 143719.203 & +380437.85 & $*$ & 17.49 & 17.46 & -0.02 & 0.02 & 0.16 & 34.25 & 35.08 & -26.9 & 32.9 & 1.72 & 1.043 & \\
\hline 143739.500 & +311901.59 & $*$ & 17.75 & 18.05 & 0.29 & 0.03 & 0.62 & 60.37 & 60.32 & -26.9 & 33.4 & 2.16 & 1.366 & o,No s \\
\hline 143756.463 & +351936.47 & $*$ & 17.49 & 17.71 & 0.22 & 0.03 & 0.61 & 16.53 & 53.48 & -25.1 & 32.5 & 2.06 & 0.537 & o \\
\hline 143848.244 & +310605.01 & $\mathrm{gO}$ & 17.74 & 18.73 & 0.99 & 0.04 & 1.07 & 1.01 & 0.74 & -24.8 & 31.1 & 0.72 & 0.737 & \\
\hline 143849.824 & +360629.61 & $*$ & 17.74 & 18.69 & 0.95 & 0.03 & 0.05 & 4.20 & 4.20 & -26.2 & 32.2 & 1.26 & 1.326 & \\
\hline 143910.820 & +245503.28 & $*$ & 17.60 & 18.18 & 0.59 & 0.09 & 0.13 & 22.48 & 24.27 & -27.3 & 33.1 & 1.79 & 1.646 & \\
\hline 143920.583 & +371202.96 & $*$ & 17.03 & 17.18 & 0.15 & 0.03 & 0.51 & 54.22 & 56.71 & -27.1 & 33.1 & 1.82 & 1.021 & \\
\hline 144050.099 & +333350.23 & $\mathrm{gE}$ & 17.73 & 18.27 & 0.54 & 0.03 & 0.85 & 5.71 & 5.44 & -27.3 & 32.6 & 1.19 & 1.763 & \\
\hline 144116.812 & +271634.45 & $*$ & 17.04 & 17.46 & 0.41 & 0.06 & 0.32 & 42.49 & 44.44 & -27.3 & 33.2 & 1.80 & 1.252 & \\
\hline 144253.304 & +420549.67 & $*$ & 17.49 & 18.34 & 0.85 & 0.03 & 0.24 & 11.95 & 11.83 & -26.5 & 32.6 & 1.58 & 1.273 & \\
\hline 144733.052 & +345506.82 & $\mathrm{gO}$ & 16.88 & 17.34 & 0.45 & 0.04 & 0.17 & 14.02 & 14.44 & -25.9 & 32.1 & 1.33 & 0.659 & $\mathrm{X}, \mathrm{I}$ \\
\hline 144825.098 & +355947.16 & $\mathrm{gE}$ & 14.95 & 16.06 & 1.11 & 0.03 & 0.27 & 1.44 & 1.48 & -23.2 & 29.6 & -0.09 & 0.113 & X,I \\
\hline 145012.144 & +302551.61 & $*$ & 17.66 & 18.81 & 1.16 & 0.05 & 0.26 & 10.19 & 10.60 & -25.9 & 32.5 & 1.72 & 1.220 & \\
\hline 145331.479 & +264946.88 & $*$ & 16.84 & 17.86 & 1.02 & 0.09 & 0.10 & 14.23 & 14.31 & -23.4 & 31.4 & 1.59 & 0.279 & \\
\hline 145342.862 & +233400.43 & $*$ & 17.71 & 18.61 & 0.90 & 0.09 & 0.26 & 3.28 & 4.86 & -24.5 & 31.6 & 1.37 & 0.614 & \\
\hline 145439.600 & +334904.15 & $*$ & 17.28 & 19.00 & 1.72 & 0.04 & 0.31 & 4.41 & 4.17 & -25.6 & 32.1 & 1.42 & 1.140 & \\
\hline 145543.419 & +300322.48 & $*$ & 16.15 & 16.59 & 0.44 & 0.06 & 0.61 & 1.80 & 1.41 & -26.5 & 31.2 & 0.13 & 0.626 & $\mathrm{P} 1$ \\
\hline 145705.181 & +270757.26 & $*$ & 16.64 & 16.85 & 0.21 & 0.08 & 0.51 & 1.64 & 1.22 & -29.6 & 32.3 & 0.03 & 2.522 & \\
\hline 145924.251 & +340113.11 & $*$ & 17.68 & 17.97 & 0.29 & 0.04 & 0.11 & 20.94 & 21.73 & -28.7 & 33.5 & 1.58 & 2.783 & \\
\hline 145958.475 & +33 3701.57 & $*$ & 17.01 & 17.11 & 0.10 & 0.04 & 0.33 & 7.22 & 11.57 & -26.0 & 32.0 & 1.15 & 0.635 & $\mathrm{o}, \mathrm{X}$ \\
\hline
\end{tabular}


TABLE 2-Continued

\begin{tabular}{|c|c|c|c|c|c|c|c|c|c|c|c|c|c|c|}
\hline $\begin{array}{l}\text { RA } \\
\text { (1) }\end{array}$ & $\begin{array}{c}\text { Dec } \\
(2)\end{array}$ & $\begin{array}{l}\mathrm{Cl} \\
(3)\end{array}$ & $\begin{array}{c}E \\
(4)\end{array}$ & $\begin{array}{c}\mathrm{O} \\
(5)\end{array}$ & $\begin{array}{c}\text { O-E } \\
(6)\end{array}$ & $\begin{array}{c}\mathrm{A}(\mathrm{E}) \\
(7)\end{array}$ & $\begin{array}{c}\text { Sep } \\
(8)\end{array}$ & $\begin{array}{l}S_{p} \\
(9)\end{array}$ & $\begin{array}{c}S_{i} \\
(10)\end{array}$ & $\begin{array}{c}M_{B} \\
(11)\end{array}$ & $\begin{array}{c}\log L_{R} \\
\text { (12) }\end{array}$ & $\begin{array}{c}\log R^{*} \\
\text { (13) }\end{array}$ & $\begin{array}{c}\mathrm{Z} \\
(14)\end{array}$ & $\begin{array}{c}\text { Notes } \\
(15)\end{array}$ \\
\hline 150321.388 & 233059.52 & $*$ & 17.49 & 17.67 & 0.18 & 0.12 & 0.45 & 2.24 & 1.82 & -24.4 & 30.9 & 0.69 & 0.404 & \\
\hline 150737.494 & +230432.52 & $\mathrm{gE}$ & 17.40 & 18.20 & 0.80 & 0.14 & 0.41 & 17.67 & 17.81 & -24.2 & 31.9 & 1.79 & 0.465 & \\
\hline 150911.242 & +215508.65 & $*$ & 16.05 & 17.08 & 1.03 & 0.13 & 0.23 & 8.30 & 8.60 & -25.2 & 31.6 & 1.03 & 0.435 & \\
\hline 151402.520 & +281334.68 & $*$ & 17.06 & 17.73 & 0.67 & 0.09 & 0.22 & 63.29 & 65.05 & -27.6 & 33.5 & 2.05 & 1.545 & \\
\hline 151443.045 & +365050.44 & $*$ & 15.58 & 15.41 & -0.18 & 0.06 & 0.07 & 48.97 & 70.99 & -26.5 & 32.3 & 1.29 & 0.369 & $\mathrm{o}, \mathrm{X}$ \\
\hline 151728.497 & +285615.96 & $*$ & 17.11 & 17.66 & 0.56 & 0.07 & 0.28 & 1.39 & 0.84 & -22.9 & 30.1 & 0.51 & 0.208 & $\mathrm{P} 1$ \\
\hline 151740.487 & +361215.16 & $*$ & 17.68 & 18.40 & 0.72 & 0.05 & 1.02 & 73.77 & 75.96 & -26.6 & 33.5 & 2.40 & 1.368 & \\
\hline 151936.152 & +283827.93 & $*$ & 16.51 & 17.20 & 0.70 & 0.07 & 0.53 & 2.08 & 1.99 & -24.0 & 30.5 & 0.49 & 0.269 & $\mathrm{P} 1$ \\
\hline 152314.434 & +375928.71 & $*$ & 17.37 & 18.41 & 1.04 & 0.04 & 0.65 & 1.67 & 1.83 & -26.5 & 31.9 & 0.78 & 1.344 & HiBAL \\
\hline 152350.435 & +391404.83 & $*$ & 15.93 & 16.93 & 1.00 & 0.05 & 0.63 & 3.75 & 4.07 & -26.3 & 31.6 & 0.61 & 0.657 & o,LoBAL \\
\hline 152523.587 & +420116.98 & $*$ & 17.12 & 17.79 & 0.66 & 0.07 & 0.46 & 104.19 & 122.19 & -26.9 & 33.6 & 2.38 & 1.198 & o \\
\hline 152701.830 & +245249.71 & $*$ & 16.87 & 17.48 & 0.61 & 0.12 & 0.84 & 1.40 & 1.22 & -26.7 & 31.5 & 0.33 & 0.993 & \\
\hline 152840.670 & +282530.81 & $*$ & 16.65 & 16.56 & -0.09 & 0.06 & 0.96 & 1.13 & 0.78 & -25.8 & 30.7 & -0.06 & 0.447 & $\mathrm{o}, \mathrm{X}$ \\
\hline 152918.035 & +324841.96 & $*$ & 16.81 & 17.54 & 0.73 & 0.08 & 0.30 & 28.91 & 29.62 & -27.9 & 33.2 & 1.62 & 1.652 & \\
\hline 153036.351 & +235236.92 & $*$ & 16.31 & 16.02 & -0.29 & 0.14 & 0.31 & 19.26 & 22.52 & -28.1 & 32.7 & 0.96 & 0.951 & \\
\hline 153045.754 & +383952.27 & $*$ & 16.88 & 17.37 & 0.49 & 0.04 & 0.28 & 2.28 & 2.28 & -28.6 & 32.3 & 0.41 & 2.035 & o \\
\hline 153144.833 & +395542.48 & $*$ & 17.51 & 18.07 & 0.55 & 0.06 & 0.51 & 7.81 & 8.18 & -25.5 & 32.0 & 1.36 & 0.753 & \\
\hline 153159.120 & +242047.26 & $*$ & 16.83 & 16.37 & -0.46 & 0.13 & 0.17 & 11.33 & 11.13 & -26.8 & 32.0 & 0.84 & 0.631 & \\
\hline 153714.259 & +271611.52 & $*$ & 17.07 & 17.67 & 0.60 & 0.11 & 0.14 & 2.01 & 1.56 & -28.7 & 32.4 & 0.45 & 2.465 & \\
\hline 153731.022 & +335836.43 & $*$ & 17.17 & 17.74 & 0.57 & 0.08 & 0.46 & 5.27 & 5.58 & -26.6 & 32.1 & 1.03 & 1.025 & \\
\hline 153901.541 & +220621.11 & $*$ & 16.53 & 17.25 & 0.71 & 0.14 & 0.92 & 1.67 & 1.56 & -27.9 & 31.9 & 0.27 & 1.478 & \\
\hline 154037.650 & +305335.26 & $*$ & 17.02 & 17.47 & 0.45 & 0.07 & 0.27 & 1.22 & 0.54 & -26.5 & 31.3 & 0.28 & 0.895 & \\
\hline 154042.984 & +413816.63 & $*$ & 16.64 & 17.34 & 0.70 & 0.08 & 0.23 & 17.67 & 18.28 & -29.1 & 33.4 & 1.27 & 2.506 & \\
\hline 154106.740 & +245302.11 & $*$ & 17.43 & 17.79 & 0.36 & 0.13 & 0.15 & 21.79 & 22.41 & -28.0 & 33.2 & 1.58 & 1.894 & \\
\hline 154159.422 & +261242.46 & $*$ & 17.24 & 17.64 & 0.41 & 0.12 & 0.26 & 15.24 & 15.24 & -26.5 & 32.5 & 1.44 & 0.971 & \\
\hline 154227.020 & +294202.50 & $*$ & 17.50 & 18.06 & 0.55 & 0.07 & 0.04 & 1.54 & 1.35 & -24.3 & 30.9 & 0.67 & 0.457 & $\mathrm{P} 1$ \\
\hline 154241.141 & +293429.22 & $\mathrm{gE}$ & 17.74 & 18.13 & 0.39 & 0.07 & 0.29 & 1.58 & 1.81 & -26.3 & 31.7 & 0.69 & 1.098 & $\mathrm{P} 1$ \\
\hline 154348.599 & +401324.86 & $*$ & 16.34 & 17.23 & 0.89 & 0.04 & 0.25 & 3.24 & 2.75 & -24.3 & 30.9 & 0.69 & 0.318 & $\mathrm{X}$ \\
\hline 154405.667 & +324048.56 & $*$ & 17.14 & 18.16 & 1.02 & 0.08 & 0.62 & 210.79 & 213.88 & -26.2 & 33.7 & 2.78 & 1.047 & \\
\hline 154445.028 & +371308.97 & $*$ & 17.48 & 18.13 & 0.65 & 0.05 & 0.55 & 602.99 & 628.57 & -26.0 & 34.1 & 3.25 & 0.971 & o \\
\hline 154510.997 & +345246.94 & $\mathrm{gO}$ & 16.50 & 16.19 & -0.31 & 0.08 & 0.81 & 14.71 & 15.93 & -26.5 & 32.0 & 0.93 & 0.518 & $\mathrm{o}, \mathrm{X}$ \\
\hline 154512.931 & +300508.35 & $*$ & 17.67 & 19.07 & 1.40 & 0.08 & 0.14 & 6.73 & 6.26 & -22.5 & 31.2 & 1.74 & 0.322 & $\mathrm{P} 1$ \\
\hline 154817.924 & +351128.37 & $*$ & 17.62 & 18.28 & 0.66 & 0.06 & 0.68 & 140.94 & 141.51 & -24.2 & 32.9 & 2.72 & 0.479 & o \\
\hline 155147.416 & +330007.93 & $*$ & 17.44 & 17.85 & 0.42 & 0.07 & 0.75 & 1.78 & 1.16 & -24.3 & 30.9 & 0.66 & 0.422 & $X$ \\
\hline 155211.336 & +231223.91 & $*$ & 17.72 & 17.86 & 0.14 & 0.16 & 0.24 & 48.40 & 51.57 & -27.2 & 33.4 & 2.01 & 1.424 & \\
\hline 155219.148 & +363435.53 & $*$ & 17.78 & 18.62 & 0.83 & 0.06 & 0.31 & 35.73 & 40.54 & -26.6 & 33.3 & 2.20 & 1.500 & \\
\hline 155235.416 & +323357.56 & $*$ & & & 0.70 & 0.06 & 1.19 & 1.27 & 0.68 & -23.6 & 30.6 & 0.72 & 0.375 & \\
\hline 155429.403 & +300119.04 & $*$ & 17.09 & 17.93 & 0.85 & 0.09 & 0.16 & 40.04 & 41.22 & -28.7 & 33.8 & 1.85 & 2.686 & $\mathrm{P} 1$ \\
\hline 155435.232 & +324647.17 & $*$ & 17.44 & 18.06 & 0.63 & 0.07 & 0.60 & 1.73 & 1.37 & -24.8 & 31.1 & 0.71 & 0.566 & \\
\hline 155507.214 & +243818.91 & $\mathrm{gO}$ & & 17.29 & -0.01 & 0.14 & 0.86 & 1.02 & 1.32 & -27.3 & 31.6 & 0.22 & 1.155 & \\
\hline 155657.725 & +425944.11 & $\mathrm{gE}$ & 17.25 & 17.96 & 0.70 & 0.04 & 0.19 & 68.79 & 73.24 & -27.6 & 33.7 & 2.17 & 1.740 & \\
\hline 155729.939 & +3304 47.01 & $*$ & 17.56 & 18.43 & 0.87 & 0.10 & 0.25 & 77.06 & 85.98 & -25.7 & 33.2 & 2.51 & 0.946 & o \\
\hline 155755.518 & +40073 & $*$ & & 18.05 & 0.27 & 0.04 & 0.14 & 73.78 & 78.95 & -26.4 & & 2.30 & 1.116 & \\
\hline 155855.160 & +332318.80 & $*$ & 16.87 & 18.17 & 1.30 & 0.08 & 0.30 & 142.96 & 144.89 & -27.3 & 33.9 & 2.56 & 1.655 & o \\
\hline 160226.882 & +274141.59 & $*$ & 17.35 & 18.19 & 0.84 & 0.11 & 0.08 & 15.87 & 17.83 & -25.9 & 32.5 & 1.73 & 0.934 & \\
\hline 160257.366 & +303851.99 & $*$ & 17.70 & 18.43 & 0.73 & 0.08 & 0.14 & 1.23 & 1.39 & -25.3 & 31.3 & 0.73 & 0.809 & $\mathrm{P} 1$ \\
\hline 160354.159 & +300208.88 & $*$ & 17.36 & 18.02 & 0.65 & 0.10 & 0.22 & 53.69 & 54.18 & -27.9 & 33.7 & 2.04 & 2.026 & $\mathrm{o}, \mathrm{Hi}$ \\
\hline 160648.178 & +291048.43 & $*$ & 17.26 & 18.59 & 1.34 & 0.10 & 1.04 & 1.26 & 0.74 & -23.0 & 30.5 & 0.82 & 0.328 & $\mathrm{P} 1$ \\
\hline 160738.345 & +240926.78 & $*$ & 17.39 & 17.76 & 0.37 & 0.17 & 0.50 & 1.04 & 0.84 & -26.8 & 31.5 & 0.30 & 1.170 & \\
\hline 160839.099 & +354226.74 & $*$ & 17.69 & 18.25 & 0.56 & 0.06 & 0.30 & 1.63 & 1.65 & -26.1 & 31.6 & 0.71 & 1.044 & \\
\hline 161047.731 & +330337.66 & $\mathrm{gE}$ & 14.36 & 15.47 & 1.11 & 0.05 & 0.82 & 6.86 & 6.21 & -23.4 & 30.2 & 0.35 & 0.097 & $\mathrm{o}, \mathrm{X}$ \\
\hline 161117.929 & +393350.21 & $*$ & 17.56 & 18.27 & 0.70 & 0.03 & 1.10 & 1.00 & 2.04 & -27.3 & 32.1 & 0.74 & 1.722 & \\
\hline 161123.237 & +295947.48 & $*$ & 17.63 & 18.76 & 1.12 & 0.08 & 0.38 & 1.69 & 1.90 & -26.8 & 32.1 & 0.91 & 1.711 & $\mathrm{P} 1$ \\
\hline 161318.000 & +293516.52 & $*$ & 17.77 & 18.60 & 0.82 & 0.10 & 0.78 & 1.10 & 1.06 & -26.8 & 31.8 & 0.61 & 1.632 & \\
\hline 161341.058 & +341247.83 & $*$ & 17.67 & 17.90 & 0.23 & 0.05 & 0.18 & 3532.04 & 3605.54 & -27.2 & 35.2 & 3.87 & 1.404 & o \\
\hline 161436.828 & +283906.03 & $\mathrm{gO}$ & 17.33 & 19.16 & 1.83 & 0.14 & 0.25 & 2.22 & 1.31 & -22.4 & 30.7 & 1.29 & 0.316 & $\mathrm{P} 1$ \\
\hline 161441.694 & +231240.71 & $*$ & 16.50 & 17.48 & 0.97 & 0.31 & 0.23 & 4.55 & 4.33 & -23.9 & 31.0 & 0.94 & 0.295 & \\
\hline 161446.956 & +374607.32 & $*$ & 16.08 & 16.64 & 0.56 & 0.04 & 0.21 & 49.26 & 51.11 & -28.6 & 33.4 & 1.50 & 1.532 & \\
\hline 161602.577 & +302339.81 & $*$ & 17.77 & 18.87 & 1.10 & 0.07 & 0.12 & 253.09 & 256.59 & -27.5 & 34.5 & 3.03 & 2.453 & \\
\hline 161806.311 & +422531.88 & $*$ & 17.50 & 18.55 & 1.05 & 0.03 & 0.24 & 27.18 & 28.30 & -25.5 & 32.7 & 2.07 & 0.937 & \\
\hline 161902.507 & +303051.47 & $\mathrm{gE}$ & 16.33 & 17.43 & 1.10 & 0.08 & 0.64 & 66.82 & 67.58 & -27.4 & 33.4 & 1.97 & 1.284 & $\mathrm{P} 1$ \\
\hline 162023.345 & +252420.35 & $*$ & 16.63 & 17.84 & 1.21 & 0.14 & 0.96 & 1.18 & 1.23 & -21.8 & 29.7 & 0.54 & 0.138 & \\
\hline 162219.851 & +320424.68 & $*$ & 17.30 & 17.83 & 0.53 & 0.06 & 0.73 & 1.36 & 0.91 & -26.3 & 31.4 & 0.46 & 0.968 & \\
\hline 162307.368 & +300406.39 & $\mathrm{gO}$ & 16.73 & 16.95 & 0.22 & 0.08 & 0.15 & 1.65 & 1.04 & -27.7 & 31.7 & 0.17 & 1.165 & $\mathrm{P} 1$ \\
\hline 162318.923 & +402258.29 & $*$ & 17.31 & 17.75 & 0.44 & 0.02 & 0.22 & 8.63 & 8.66 & -26.3 & 32.2 & 1.24 & 0.907 & o \\
\hline 162319.933 & +411702.80 & $*$ & 16.34 & 17.13 & 0.79 & 0.02 & 0.27 & 3.23 & 2.98 & -28.3 & 32.3 & 0.49 & 1.618 & o \\
\hline 162330.567 & +355933.12 & $*$ & 17.75 & 18.13 & 0.38 & 0.03 & 0.39 & 259.58 & 266.83 & -25.8 & 33.6 & 2.89 & 0.867 & o \\
\hline 162422.001 & +392441.02 & $\mathrm{gO}$ & 17.58 & 18.22 & 0.64 & 0.02 & 0.16 & 132.32 & 144.52 & -26.3 & 33.6 & 2.63 & 1.117 & o \\
\hline 162548.793 & +264658.76 & $*$ & 16.79 & 16.76 & -0.04 & 0.09 & 0.05 & 10.12 & 9.69 & -29.7 & 33.1 & 0.78 & 2.521 & o \\
\hline 162659.251 & +301534.96 & $*$ & 16.93 & 17.69 & 0.76 & 0.07 & 0.15 & 5.34 & 5.42 & -27.7 & 32.5 & 0.95 & 1.582 & $\mathrm{P} 1$ \\
\hline 162751.993 & +222404.76 & $\mathrm{gE}$ & 17.70 & 18.02 & 0.31 & 0.13 & 0.30 & 2.65 & 2.43 & -26.9 & 32.0 & 0.79 & 1.313 & \\
\hline
\end{tabular}


TABLE 2-Continued

\begin{tabular}{|c|c|c|c|c|c|c|c|c|c|c|c|c|c|c|}
\hline $\begin{array}{l}\text { RA } \\
\text { (1) }\end{array}$ & $\begin{array}{l}\text { Dec } \\
(2)\end{array}$ & $\begin{array}{l}\mathrm{Cl} \\
(3)\end{array}$ & $\begin{array}{c}E \\
(4)\end{array}$ & $\begin{array}{c}\mathrm{O} \\
(5)\end{array}$ & $\begin{array}{l}\text { O-E } \\
(6)\end{array}$ & $\begin{array}{c}\mathrm{A}(\mathrm{E}) \\
(7)\end{array}$ & $\begin{array}{c}\text { Sep } \\
(8)\end{array}$ & $\begin{array}{l}S_{p} \\
(9)\end{array}$ & $\begin{array}{c}S_{i} \\
(10)\end{array}$ & $\begin{array}{l}M_{B} \\
(11)\end{array}$ & $\begin{array}{c}\log L_{R} \\
\text { (12) }\end{array}$ & $\begin{array}{c}\log R^{*} \\
(13)\end{array}$ & $\begin{array}{c}\mathrm{Z} \\
(14)\end{array}$ & $\begin{array}{l}\text { Notes } \\
(15)\end{array}$ \\
\hline 162901.315 & 400759.62 & $*$ & 17.70 & 17.99 & 0.30 & 0.02 & 0.00 & 11.97 & 11.94 & -23.2 & $31 . J$ & 1.57 & 0.271 & $\mathrm{o}, \mathrm{X}$ \\
\hline 163020.768 & +375656.49 & $*$ & 17.60 & 17.39 & -0.21 & 0.03 & 0.05 & 20.00 & 21.73 & -24.7 & 31.9 & 1.57 & 0.395 & o \\
\hline 163206.185 & +265112.91 & $*$ & 17.61 & 18.25 & 0.64 & 0.15 & 0.54 & 9.95 & 10.14 & -27.2 & 32.8 & 1.44 & 1.628 & \\
\hline 163221.067 & +264353.45 & $*$ & 17.47 & 17.77 & 0.30 & 0.14 & 0.45 & 301.88 & 314.48 & -28.8 & 34.7 & 2.67 & 2.673 & \\
\hline 163223.757 & +291442.19 & $*$ & 17.46 & 18.01 & 0.55 & 0.06 & 0.46 & 5.56 & 5.32 & -23.6 & 31.1 & 1.23 & 0.326 & \\
\hline 163302.096 & +392427.62 & $*$ & 15.60 & 16.60 & 1.00 & 0.02 & 0.34 & 41.29 & 54.38 & -27.7 & 33.1 & 1.57 & 1.021 & o \\
\hline 163302.680 & +234928.64 & $*$ & 15.35 & 15.97 & 0.62 & 0.21 & 0.18 & 3.62 & 3.44 & -27.8 & 31.7 & 0.16 & 0.822 & $\mathrm{o}, \mathrm{X}$ \\
\hline 163323.580 & +354408.35 & $*$ & 17.50 & 17.94 & 0.44 & 0.05 & 0.88 & 1.42 & 0.93 & -27.6 & 31.9 & 0.46 & 1.692 & \\
\hline 163348.924 & +313411.66 & $*$ & 16.98 & 17.31 & 0.32 & 0.08 & 0.75 & 1.79 & 2.07 & -27.9 & 32.0 & 0.38 & 1.516 & \\
\hline 163412.779 & +3203 35.45 & $*$ & 16.58 & 16.93 & 0.35 & 0.08 & 0.05 & 175.25 & 176.52 & -29.4 & 34.3 & 2.10 & 2.341 & \\
\hline 163515.483 & +380804.56 & $*$ & 17.78 & 18.05 & 0.27 & 0.03 & 0.14 & 2653.87 & 2694.06 & -27.6 & 35.3 & 3.77 & 1.811 & o \\
\hline 163709.325 & +414030.63 & $*$ & 16.76 & 17.19 & 0.43 & 0.02 & 0.09 & 7.37 & 7.11 & -26.4 & 32.0 & 0.96 & 0.765 & o \\
\hline 163755.228 & +250930.49 & $*$ & 17.58 & 17.80 & 0.22 & 0.10 & 0.36 & 26.99 & 27.92 & -26.7 & 32.9 & 1.75 & 1.110 & \\
\hline 163931.800 & +390845.40 & $\mathrm{gE}$ & 17.00 & 17.76 & 0.76 & 0.03 & 0.25 & 1.27 & 0.72 & -22.0 & 29.8 & 0.52 & 0.143 & $X$ \\
\hline 164125.123 & +355020.29 & $\mathrm{gO}$ & 17.31 & 16.90 & -0.41 & 0.06 & 0.20 & 1.07 & 0.63 & -28.2 & 31.7 & -0.06 & 1.438 & \\
\hline 164152.295 & +305851.79 & $*$ & 17.57 & 18.70 & 1.14 & 0.07 & 0.16 & 2.14 & 2.66 & -27.2 & 32.4 & 1.01 & 2.000 & LoBAL \\
\hline 164154.244 & +400032.00 & $*$ & 17.48 & 17.86 & 0.38 & 0.03 & 1.19 & 6.89 & 5.06 & -26.4 & 32.2 & 1.18 & 1.003 & o \\
\hline 164218.951 & +315433.83 & $*$ & 17.39 & 17.73 & 0.34 & 0.06 & 0.12 & 23.08 & 24.01 & -27.1 & 32.9 & 1.64 & 1.263 & \\
\hline 164240.405 & +252307.64 & $*$ & 17.54 & 17.65 & 0.10 & 0.16 & 0.21 & 490.22 & 504.69 & -27.9 & 34.5 & 2.89 & 1.725 & \\
\hline 164258.799 & +3948 37.16 & $*$ & 15.30 & 15.84 & 0.54 & 0.04 & 0.25 & 6050.06 & 6598.61 & -27.2 & 34.7 & 3.40 & 0.595 & $\mathrm{o}, \mathrm{X}, \mathrm{I}$ \\
\hline 164347.901 & +301108.80 & $*$ & 16.57 & 17.07 & 0.50 & 0.08 & 0.04 & 1.42 & 1.38 & -27.9 & 31.8 & 0.14 & 1.380 & \\
\hline 164442.536 & +261913.19 & $*$ & 17.02 & 17.21 & 0.19 & 0.21 & 0.22 & 87.53 & 90.80 & -22.6 & 31.6 & 2.16 & 0.145 & $\mathrm{o}, \mathrm{X}$ \\
\hline 164552.122 & +224449.97 & $*$ & 17.34 & 17.85 & 0.51 & 0.13 & 0.36 & 2.30 & 1.94 & -28.8 & 32.5 & 0.56 & 2.725 & \\
\hline 164802.393 & +24291 & $\mathrm{gO}$ & 17.50 & 18.45 & 0.95 & 0.22 & 0.30 & 259.71 & 269.91 & -25.2 & 3.6 & 3 & 0.797 & \\
\hline 165005.487 & +414032.60 & $*$ & 17.18 & 17.10 & -0.08 & 0.05 & 0.22 & 170.28 & 185.19 & -25.9 & 33.2 & 2.35 & 0.584 & $\mathrm{o}, \mathrm{X}$ \\
\hline 165137.565 & +400218.71 & $*$ & 16.92 & 17.18 & 0.25 & 0.05 & 0.53 & 42.73 & 43.90 & -29.1 & 33.7 & 1.60 & 2.316 & \\
\hline 165252.591 & +360057.21 & $*$ & 17.54 & 18.82 & 1.28 & 0.04 & 0.59 & 1.36 & 2.22 & -22.4 & 30.6 & 1.16 & 0.281 & \\
\hline 165255.932 & +312343.86 & $\mathrm{gE}$ & 17.69 & 17.57 & -0.12 & 0.08 & 0.35 & 1.16 & 1.47 & -25.4 & 31.1 & 0.44 & 0.590 & \\
\hline 165500.223 & +303040.60 & $*$ & 17.16 & 17.27 & 0.11 & 0.09 & 0.38 & 3.20 & 2.86 & -24.8 & 31.1 & 0.68 & 0.405 & \\
\hline & & $*$ & 17.73 & & 0.63 & 0.13 & 0.60 & 2.06 & 1.99 & -26.4 & .8 & 3 & 39 & \\
\hline 165543.235 & +394519.91 & $*$ & 17.74 & 18.11 & 0.37 & 0.05 & 0.13 & 10.15 & 10.16 & -27.5 & 32.8 & 1.38 & 1.747 & $\mathrm{HiB}$ \\
\hline 165557.366 & +321805.91 & $*$ & 17.25 & 17.80 & 0.55 & 0.08 & 0.90 & 1.10 & 1.19 & -27.1 & 31.7 & 0.35 & 1.327 & \\
\hline .976 & & $*$ & 17.35 & 17.86 & 0.51 & 0.20 & 0.98 & 1.72 & 1.16 & -25.5 & 1.3 & .61 & 0.706 & \\
\hline 165722.105 & +395551.61 & $*$ & 17.39 & 17.73 & 0.34 & 0.05 & 0.30 & 1.19 & 0.77 & -25.2 & 31.0 & 0.41 & 0.579 & \\
\hline 165744.598 & +425731.31 & $*$ & 17.56 & 17.82 & 0.26 & 0.06 & 0.61 & 1.10 & 1.28 & -26.4 & 31.4 & 0.43 & 0.992 & \\
\hline 165801.401 & +344328.51 & $*$ & 17.77 & 17.78 & 0.01 & 0.05 & 0.43 & 406.35 & 444.06 & -28.0 & 34.5 & 2.87 & 1.925 & o \\
\hline 165924.186 & +262937.29 & $\mathrm{gE}$ & 17.78 & 18.02 & 0.24 & 0.12 & 0.68 & 391.07 & 538.46 & -25.7 & 33.9 & 3.16 & 0.794 & o \\
\hline 165931.926 & +373528.97 & $*$ & 17.21 & 17.14 & -0.07 & 0.06 & 0.15 & 18.31 & 18.57 & -26.5 & 32.4 & 1.34 & 0.775 & \\
\hline 170036.332 & +341414.91 & $*$ & 17.35 & 17.24 & -0.11 & 0.06 & 0.21 & 1.16 & 0.70 & -26.4 & 31.2 & 0.18 & 0.796 & \\
\hline 170158.260 & +222641.49 & $\mathrm{gE}$ & 16.68 & 18.42 & 1.74 & 0.14 & 0.50 & 5.90 & 5.48 & -22.0 & 30.7 & 1.44 & 0.197 & \\
\hline 170231.030 & +324719.65 & $*$ & 15.84 & 15.74 & -0.10 & 0.06 & 0.35 & 1.82 & 1.52 & -24.3 & 30.0 & -0.13 & 0.163 & $\mathrm{o}, \mathrm{X}$ \\
\hline 170248.890 & +421632.99 & $*$ & 17.26 & 18.22 & 0.96 & 0.05 & 0.16 & 3.63 & 3.72 & -25.0 & 31.5 & 1.09 & 0.653 & \\
\hline 170322.806 & +275712.93 & $*$ & 17.72 & 17.92 & 0.20 & 0.22 & 0.48 & 1.19 & 0.97 & -25.3 & 31.1 & 0.48 & 0.666 & \\
\hline 170446.476 & +380206.53 & $*$ & 16.27 & 16.58 & 0.31 & 0.08 & 0.71 & 2.57 & 2.14 & -21.3 & 29.4 & 0.37 & 0.063 & \\
\hline 170634.158 & +361508.01 & $*$ & 17.72 & 17.98 & 0.26 & 0.08 & 0.21 & 18.85 & 19.19 & -26.0 & 32.6 & 1.68 & 0.918 & o,new $z$ \\
\hline 170648.061 & +321422.68 & $*$ & 16.28 & 16.71 & 0.43 & 0.11 & 0.28 & 36.32 & 38.71 & -27.7 & 33.0 & 1.46 & 1.070 & o,new $z$ \\
\hline 170804.245 & & $\mathrm{gO}$ & & & 1.18 & 0.11 & 0.14 & 1.71 & 2.06 & -22.6 & 30.7 & 1.16 & 0.312 & \\
\hline 170823.134 & +412309.53 & $*$ & 16.79 & 17.11 & 0.33 & 0.07 & 0.43 & 1.23 & 0.94 & -26.7 & 31.3 & 0.15 & 0.837 & \\
\hline 170906.105 & +420157.49 & $*$ & 17.57 & 18.19 & 0.61 & 0.06 & 0.54 & 5.61 & 5.67 & -23.3 & 31.1 & 1.31 & 0.307 & \\
\hline 171013.430 & +334402.76 & $\mathrm{gE}$ & & & 0.74 & 0.07 & 0.82 & 4.05 & 4.60 & -24.7 & 30.7 & 0.32 & 0.208 & $\mathrm{o}, \mathrm{X}$ \\
\hline 171304.476 & +352333.43 & $*$ & 15.74 & 16.73 & 0.99 & 0.08 & 0.24 & 11.13 & 11.24 & -21.8 & 30.2 & 1.07 & 0.083 & $\mathrm{X}$ \\
\hline 171550.369 & +410337.47 & $\mathrm{gO}$ & 17.56 & 17.49 & -0.06 & 0.08 & 0.24 & 1.28 & 0.77 & -25.7 & 31.1 & 0.34 & 0.650 & \\
\hline 171601.918 & +311213.79 & $\mathrm{gE}$ & 14.47 & & 0.67 & 0.09 & 0.19 & 2.68 & 2.42 & -24.0 & .9 & -0.20 & 0.110 & $\mathrm{o}, \mathrm{X}, \mathrm{I}$ \\
\hline 171653.670 & +230553.44 & $*$ & 17.04 & 17.55 & 0.51 & 0.12 & 0.54 & 34.07 & 34.42 & -27.7 & 33.2 & 1.70 & 1.536 & \\
\hline 171654.191 & +302701.53 & $*$ & 16.54 & 16.83 & 0.28 & 0.12 & 0.19 & 4.25 & 3.96 & -26.7 & 31.7 & 0.58 & 0.751 & \\
\hline 171728.606 & +245256.59 & $\mathrm{gO}$ & 15.53 & 16.22 & 0.69 & 0.14 & 0.36 & 1.65 & 1.60 & -26.0 & 30.8 & -0.03 & 0.434 & \\
\hline 171820.922 & +424914.05 & $\mathrm{gE}$ & 17.34 & 17.77 & 0.44 & 0.06 & 0.14 & 2.55 & 2.44 & -22.3 & 30.2 & 0.83 & 0.167 & \\
\hline 171850.343 & +304201.31 & $*$ & 17.44 & 18.26 & 0.81 & 0.11 & 0.49 & 1.25 & 0.64 & -23.0 & 30.3 & 0.69 & 0.280 & \\
\hline 171934.182 & +251058.46 & $\mathrm{gE}$ & 15.38 & 16.61 & 1.23 & 0.14 & 0.33 & 12.67 & 13.83 & -26.3 & 32.0 & 1.03 & 0.579 & o \\
\hline 171938.732 & +291854.85 & $*$ & 17.18 & 17.95 & 0.77 & 0.10 & 1.04 & 6.23 & 7.53 & -26.7 & 32.4 & 1.23 & 1.197 & \\
\hline 172007.694 & +365439.24 & $*$ & 16.69 & 17.63 & 0.94 & 0.13 & 0.04 & 3.42 & 3.34 & -24.0 & 30.9 & 0.87 & 0.332 & \\
\hline 172109.519 & +354215.74 & $*$ & 16.42 & 17.02 & 0.60 & 0.11 & 0.60 & 386.51 & 392.99 & -24.3 & 32.9 & 2.69 & 0.283 & $o$, new $z, X$ \\
\hline 172320.803 & +341757.86 & $\mathrm{gE}$ & 14.82 & 15.04 & 0.22 & 0.10 & 0.45 & 438.57 & 441.20 & -25.5 & 32.6 & 1.97 & 0.205 & $\mathrm{o}, \mathrm{X}$ \\
\hline 172354.273 & +374841.62 & $*$ & 17.44 & 18.22 & 0.78 & 0.11 & 0.51 & 1.70 & 1.72 & -25.6 & 31.4 & 0.74 & 0.828 & \\
\hline 172635.129 & +321322.89 & $*$ & 17.05 & 17.53 & 0.48 & 0.12 & 0.14 & 120.94 & 123.67 & -26.9 & 33.5 & 2.29 & 1.094 & o \\
\hline 172824.109 & +424508.99 & $*$ & 17.62 & 18.59 & 0.97 & 0.06 & 0.53 & 1.27 & 0.70 & -25.0 & 31.2 & 0.76 & 0.768 & \\
\hline 172859.130 & +383826.36 & $*$ & 16.61 & 16.76 & 0.15 & 0.10 & 0.11 & 240.27 & 245.41 & -28.3 & 34.0 & 2.25 & 1.391 & 0 \\
\hline 173403.469 & +403753.11 & $*$ & 15.91 & 16.27 & 0.36 & 0.12 & 1.00 & 1.07 & 2.53 & -25.5 & 30.9 & 0.19 & 0.356 & \\
\hline
\end{tabular}

NOTE.-Descriptions of table columns:

Cols 1-2: FIRST radio positions (J2000).

Col 3: Optical morphology in the APM catalog. *: stellar on both plates, gE: nonstellar on E plate, gO: nonstellar on O plate.

Cols 4-6: Extinction-corrected APM magnitudes and color.

Col 7: Extinction correction (magnitudes) that was applied to E. The correction for O is 1.63 times larger.

Col 8: Separation between radio and optical positions (arcsec.) 
TABLE 3

NARROW-LINE AGN IN FBQS CANDIDATE LIST

\begin{tabular}{|c|c|c|c|c|c|c|c|c|c|c|c|c|c|c|}
\hline $\begin{array}{l}\text { RA } \\
\text { (1) }\end{array}$ & $\begin{array}{c}\text { Dec } \\
\text { (2) }\end{array}$ & $\begin{array}{l}\mathrm{Cl} \\
(3)\end{array}$ & $\begin{array}{c}E \\
(4)\end{array}$ & $\begin{array}{l}\mathrm{O} \\
(5)\end{array}$ & $\begin{array}{l}\text { O-E } \\
(6)\end{array}$ & $\begin{array}{c}\mathrm{A}(\mathrm{E}) \\
(7)\end{array}$ & $\begin{array}{l}\text { Sep } \\
(8)\end{array}$ & $\begin{array}{l}S_{p} \\
(9)\end{array}$ & $\begin{array}{c}S_{i} \\
(10)\end{array}$ & $\begin{array}{c}M_{B} \\
(11)\end{array}$ & $\begin{array}{c}\log L_{R} \\
\text { (12) }\end{array}$ & $\begin{array}{c}\log R^{*} \\
\text { (13) }\end{array}$ & $\begin{array}{c}\mathrm{Z} \\
(14)\end{array}$ & $\begin{array}{l}\text { Notes } \\
\text { (15) }\end{array}$ \\
\hline 070625.149 & +390151.58 & * & 16.73 & 17.65 & 0.93 & 0.26 & 0.12 & 5.58 & 5.44 & -21.0 & 30.0 & 1.13 & 0.086 & \\
\hline 071003.644 & +421703.07 & $\mathrm{gO}$ & 16.95 & 18.87 & 1.92 & 0.19 & 0.53 & 2.05 & 1.69 & -20.6 & 29.9 & 1.18 & 0.124 & \\
\hline 073053.727 & +293817.52 & * & 17.33 & 18.82 & 1.49 & 0.17 & 0.85 & 1.12 & 0.81 & -21.0 & 29.8 & 0.89 & 0.150 & \\
\hline 074932.953 & +283406.66 & $\mathrm{gO}$ & 17.39 & 18.80 & 1.41 & 0.09 & 0.29 & 27.33 & 27.95 & -22.9 & 31.9 & 2.24 & 0.340 & I \\
\hline 075516.716 & +392920.20 & $*$ & 15.54 & 17.12 & 1.58 & 0.14 & 0.16 & 1.17 & 1.03 & -21.2 & 29.2 & 0.24 & 0.075 & \\
\hline 075820.974 & +392335.80 & $\mathrm{gE}$ & 17.60 & 18.37 & 0.77 & 0.16 & 0.22 & 4.40 & 4.41 & -22.3 & 30.7 & 1.29 & 0.216 & \\
\hline 075902.391 & +282756.22 & $\mathrm{gE}$ & 17.53 & 19.31 & 1.78 & 0.10 & 0.94 & 1.15 & 1.62 & -21.3 & 30.2 & 1.24 & 0.214 & \\
\hline 080408.091 & +394728.18 & $\mathrm{gE}$ & 16.99 & 18.62 & 1.64 & 0.13 & 0.76 & 1.35 & 0.72 & -20.5 & 29.6 & 0.90 & 0.110 & \\
\hline 080455.724 & +383349.59 & $\mathrm{gE}$ & 16.34 & 18.21 & 1.86 & 0.12 & 0.09 & 1.33 & 0.80 & -20.8 & 29.5 & 0.73 & 0.104 & \\
\hline 082944.336 & +222527.46 & $\mathrm{gE}$ & 9.67 & 9.83 & 0.16 & 0.11 & 0.52 & 2.18 & 2.82 & -26.1 & 28.6 & -2.28 & 0.025 & $\mathrm{I}, \mathrm{B}=15$ \\
\hline 083207.835 & +40 3452.73 & $\mathrm{gO}$ & 16.13 & 17.15 & 1.02 & 0.12 & 0.23 & 2.30 & 1.87 & -22.3 & 29.9 & 0.54 & 0.128 & I \\
\hline 083407.608 & +290839.95 & $\mathrm{gE}$ & 17.20 & 18.90 & 1.70 & 0.11 & 0.28 & 2.53 & 1.75 & -20.8 & 30.1 & 1.28 & 0.141 & \\
\hline 083726.580 & +400207.57 & $\mathrm{gO}$ & 7.94 & 9.38 & 1.44 & 0.11 & 0.95 & 2.49 & 3.39 & -26.4 & 28.6 & -2.38 & 0.024 & $\mathrm{I}, \mathrm{B}=14.7$ \\
\hline 083726.918 & +312104.71 & $\mathrm{gE}$ & 16.60 & 17.37 & 0.77 & 0.12 & 0.18 & 1.45 & 1.57 & -22.9 & 30.1 & 0.45 & 0.179 & \\
\hline 083946.113 & +225100.46 & $\mathrm{gE}$ & 17.42 & 19.03 & 1.61 & 0.10 & 0.14 & 2.29 & 2.16 & -21.2 & 30.2 & 1.28 & 0.176 & \\
\hline 084215.215 & +402533.12 & $\mathrm{gO}$ & 14.54 & 15.88 & 1.34 & 0.09 & 0.83 & 1.68 & 1.13 & -21.7 & 29.1 & -0.09 & 0.055 & I \\
\hline 084322.477 & +413349.67 & * & 17.38 & 19.06 & 1.68 & 0.09 & 0.15 & 1.77 & 1.35 & -21.5 & 30.2 & 1.17 & 0.205 & \\
\hline 084458.300 & +30 56 38.36 & $\mathrm{gE}$ & 16.88 & 18.56 & 1.68 & 0.14 & 0.35 & 1.85 & 1.48 & -19.4 & 29.2 & 1.02 & 0.065 & \\
\hline 084529.171 & +385459.76 & $\mathrm{gO}$ & 16.95 & 18.93 & 1.98 & 0.09 & 0.54 & 2.05 & 1.92 & -20.9 & 30.0 & 1.20 & 0.152 & \\
\hline 085038.142 & +303932.07 & $*$ & 17.41 & 18.84 & 1.44 & 0.10 & 0.52 & 1.86 & 1.55 & -21.3 & 30.1 & 1.12 & 0.172 & \\
\hline 085253.023 & +412533.39 & $*$ & 17.33 & 19.06 & 1.72 & 0.08 & 0.39 & 1.19 & 1.35 & -20.6 & 29.8 & 1.07 & 0.136 & \\
\hline 091336.157 & +244754.28 & $*$ & 17.47 & 18.99 & 1.52 & 0.12 & 0.25 & 7.55 & 7.60 & -20.8 & 30.6 & 1.79 & 0.147 & I \\
\hline 091443.475 & +30 5935.73 & $*$ & 17.49 & 19.40 & 1.91 & 0.06 & 0.12 & 5.38 & 5.52 & -22.1 & 31.1 & 1.79 & 0.306 & I \\
\hline 091902.230 & +261611.94 & $\mathrm{gO}$ & 9.41 & 10.88 & 1.46 & 0.09 & 1.16 & 6.36 & 8.17 & -23.0 & 28.3 & -1.40 & 0.008 & $\mathrm{I}, \mathrm{B}=14.2$ \\
\hline 092034.293 & +381934.64 & $\mathrm{gO}$ & 17.64 & 19.20 & 1.56 & 0.04 & 0.55 & 1.38 & 1.16 & -22.3 & 30.5 & 1.10 & 0.310 & \\
\hline 093925.100 & +272034.27 & $*$ & 17.76 & 19.39 & 1.62 & 0.06 & 0.13 & 1.55 & 1.14 & -19.3 & 29.4 & 1.27 & 0.089 & \\
\hline 094319.161 & +361452.31 & $\mathrm{gO}$ & 10.57 & 10.50 & -0.07 & 0.03 & 0.16 & 73.77 & 74.91 & -25.1 & 29.9 & -0.59 & 0.022 & $\mathrm{~B}=14.4$ \\
\hline 094525.885 & +352103.46 & $\mathrm{gE}$ & 17.20 & 18.22 & 1.03 & 0.03 & 0.19 & 140.45 & 147.55 & -22.3 & 32.2 & 2.76 & 0.207 & \\
\hline 103528.514 & +394629.00 & $\mathrm{gE}$ & 17.57 & 19.21 & 1.64 & 0.04 & 0.72 & 1.18 & 0.76 & -20.1 & 29.6 & 1.07 & 0.117 & \\
\hline 103942.649 & +404845.07 & * & 17.67 & 19.38 & 1.70 & 0.06 & 0.82 & 1.29 & 1.07 & -19.7 & 29.5 & 1.18 & 0.105 & \\
\hline 104000.596 & +390720.10 & $*$ & 9.17 & 8.08 & -1.09 & 0.04 & 0.05 & 11.05 & 13.15 & -28.3 & 29.5 & -2.31 & 0.031 & $\mathrm{I}, \mathrm{B}=14.7$ \\
\hline 105745.128 & +403730.99 & $\mathrm{gE}$ & 17.20 & 18.35 & 1.15 & 0.03 & 0.17 & 1.01 & 0.89 & -21.6 & 29.8 & 0.66 & 0.160 & \\
\hline 110635.617 & +353957.74 & $\mathrm{gE}$ & 16.60 & 17.94 & 1.33 & 0.05 & 0.51 & 2.84 & 3.04 & -21.2 & 29.9 & 0.98 & 0.110 & \\
\hline 110952.828 & +423315.51 & $*$ & 17.20 & 19.16 & 1.96 & 0.04 & 0.34 & 16.21 & 16.96 & -21.9 & 31.4 & 2.19 & 0.260 & I \\
\hline 111211.102 & +273256.57 & $*$ & 16.60 & 17.43 & 0.83 & 0.07 & 0.26 & 2.09 & 2.56 & -23.4 & 30.5 & 0.68 & 0.235 & $\mathrm{E}$ \\
\hline 114052.870 & +232513.63 & $\mathrm{gE}$ & 17.22 & 18.86 & 1.63 & 0.06 & 0.74 & 1.66 & 1.64 & -20.8 & 29.9 & 1.08 & 0.140 & \\
\hline 115331.189 & +223753.55 & $\mathrm{gO}$ & 17.04 & 18.32 & 1.27 & 0.08 & 0.25 & 3.61 & 4.10 & -20.8 & 30.0 & 1.26 & 0.109 & I \\
\hline 115844.661 & +413426.32 & $\mathrm{gO}$ & 17.05 & 18.45 & 1.41 & 0.04 & 0.29 & 1.18 & 1.09 & -21.1 & 29.7 & 0.77 & 0.130 & \\
\hline 120443.339 & +311038.21 & $\mathrm{gO}$ & 9.68 & 10.58 & 0.90 & 0.05 & 0.91 & 5.10 & 7.59 & -25.3 & 29.0 & -1.55 & 0.025 & $\mathrm{o}, \mathrm{B}=14.0$ \\
\hline 121609.612 & +415928.43 & $\mathrm{gE}$ & 16.34 & 17.16 & 0.81 & 0.05 & 0.16 & 18.28 & 18.42 & -23.7 & 31.4 & 1.42 & 0.240 & \\
\hline 122214.649 & +242420.82 & $\mathrm{gE}$ & 16.84 & 18.46 & 1.63 & 0.06 & 0.30 & 2.74 & 2.67 & -20.9 & 30.0 & 1.14 & 0.120 & \\
\hline 122222.413 & +241138.27 & $\mathrm{gE}$ & 16.93 & 18.82 & 1.89 & 0.05 & 0.83 & 1.33 & 2.08 & -20.4 & 29.8 & 1.17 & 0.116 & \\
\hline 122331.392 & +243140.82 & $*$ & 16.68 & 18.11 & 1.43 & 0.05 & 0.10 & 5.40 & 5.90 & -22.2 & 30.7 & 1.32 & 0.185 & \\
\hline 123016.047 & +353340.78 & $*$ & 17.10 & 18.73 & 1.63 & 0.04 & 1.00 & 1.95 & 1.70 & -21.0 & 30.0 & 1.10 & 0.144 & \\
\hline 124035.819 & +303722.82 & $\mathrm{gE}$ & 17.22 & 18.97 & 1.75 & 0.04 & 0.43 & 1.20 & 2.17 & -21.8 & 30.4 & 1.23 & 0.224 & \\
\hline 124121.705 & +313203.63 & $\mathrm{gO}$ & 16.94 & 18.17 & 1.23 & 0.04 & 0.08 & 2.83 & 2.98 & -20.0 & 29.5 & 1.07 & 0.072 & $\mathrm{X}$ \\
\hline 124417.498 & +300443.77 & $\mathrm{gE}$ & 17.51 & 19.33 & 1.82 & 0.04 & 0.12 & 1.91 & 2.01 & -21.2 & 30.3 & 1.34 & 0.205 & \\
\hline 125521.802 & +421904.90 & $\mathrm{gE}$ & 17.61 & 19.20 & 1.59 & 0.04 & 0.18 & 1.78 & 1.40 & -20.6 & 30.0 & 1.24 & 0.150 & \\
\hline 132018.246 & +390722.56 & $\mathrm{gE}$ & 17.07 & 18.72 & 1.64 & 0.03 & 0.56 & 2.16 & 1.82 & -22.2 & 30.4 & 1.12 & 0.240 & \\
\hline 133110.974 & +414631.36 & $\mathrm{gE}$ & 14.70 & 15.07 & 0.37 & 0.02 & 1.00 & 1.14 & 2.22 & -21.5 & 28.8 & -0.29 & 0.034 & I \\
\hline 134011.521 & +341817.09 & $*$ & 17.79 & 19.06 & 1.27 & 0.03 & 0.77 & 1.36 & 2.11 & -21.7 & 30.4 & 1.25 & 0.223 & \\
\hline 134321.372 & +232038.81 & $\mathrm{gE}$ & 16.35 & 18.05 & 1.70 & 0.04 & 0.28 & 1.04 & 0.95 & -22.1 & 29.8 & 0.55 & 0.173 & \\
\hline 134717.745 & +340855.32 & $*$ & 7.44 & 8.23 & 0.79 & 0.06 & 1.02 & 2.14 & 3.84 & -26.7 & 28.3 & -2.78 & 0.016 & 14.6 \\
\hline 135036.022 & +334217.53 & $\mathrm{gO}$ & 7.86 & 8.91 & 1.05 & 0.07 & 0.63 & 97.21 & 99.73 & -25.7 & 29.6 & -1.10 & 0.014 & $\mathrm{~B}=13.5$ \\
\hline 140438.767 & +263648.31 & * & 17.72 & 19.04 & 1.31 & 0.04 & 0.37 & 2.75 & 2.72 & -20.9 & 30.2 & 1.37 & 0.159 & \\
\hline 141636.927 & +243009.17 & $\mathrm{gO}$ & 8.61 & 10.44 & 1.82 & 0.05 & 0.78 & 2.00 & 2.45 & -26.4 & 28.9 & -2.10 & 0.039 & $\mathrm{I}, \mathrm{B}=15.2$ \\
\hline 143236.352 & +375251.03 & $*$ & 17.61 & 19.58 & 1.97 & 0.02 & 0.95 & 1.26 & 1.16 & -20.5 & 29.9 & 1.24 & 0.168 & \\
\hline 143301.186 & +301302.46 & $\mathrm{gE}$ & 17.21 & 18.48 & 1.27 & 0.05 & 0.6 & 2.51 & 2.75 & -20.7 & 29.9 & 1.15 & 0.110 & \\
\hline 143558.257 & +312027.33 & $\mathrm{gO}$ & 16.89 & 18.41 & 1.52 & 0.04 & 0.13 & 10.76 & 10.97 & -19.4 & 30.0 & 1.73 & 0.060 & \\
\hline 143946.932 & +250351.06 & $\mathrm{gO}$ & 17.10 & 18.43 & 1.33 & 0.08 & 0.58 & 1.53 & 2.02 & -20.9 & 29.8 & 1.00 & 0.120 & I \\
\hline 145219.762 & +265010.28 & $\mathrm{gO}$ & 17.66 & 19.28 & 1.62 & 0.09 & 0.1 & 1.03 & 0.95 & -20.5 & 29.7 & 1.04 & 0.143 & \\
\hline 145258.979 & +330005.44 & $\mathrm{gE}$ & 17.70 & 18.51 & 0.82 & 0.05 & 0.68 & 10.61 & 41.19 & -22.5 & 31.8 & 2.32 & 0.247 & \\
\hline 145325.217 & +341802.27 & $\mathrm{gO}$ & 17.39 & 18.27 & 0.87 & 0.05 & 0.29 & 4.72 & 4.82 & -21.8 & 30.5 & 1.30 & 0.162 & \\
\hline 145519.410 & +322601.52 & $\mathrm{gO}$ & 15.80 & 16.92 & 1.12 & 0.04 & 0.27 & 2.76 & 3.22 & -21.7 & 29.7 & 0.60 & 0.087 & \\
\hline 150011.399 & +285322.22 & $\mathrm{gO}$ & 16.20 & 17.94 & 1.74 & 0.05 & 0.36 & 1.87 & 1.27 & -21.5 & 29.8 & 0.77 & 0.125 & \\
\hline 150136.561 & +320713.26 & $\mathrm{gO}$ & 17.03 & 18.93 & 1.90 & 0.05 & 0.53 & 1.05 & 0.85 & -20.3 & 29.5 & 0.91 & 0.115 & \\
\hline 150427.131 & +352916.66 & $\mathrm{gE}$ & 17.12 & 19.06 & 1.94 & 0.04 & 0.62 & 1.17 & 1.07 & -20.5 & 29.7 & 1.01 & 0.131 & \\
\hline 151732.271 & +223922.77 & $\mathrm{gE}$ & 16.93 & 18.22 & 1.28 & 0.11 & 0.23 & 1.37 & 1.08 & -20.9 & 29.6 & 0.74 & 0.109 & \\
\hline 152022.470 & +263207.58 & $\mathrm{gO}$ & 16.56 & 17.95 & 1.38 & 0.10 & 0.23 & 13.86 & 13.90 & -21.4 & 30.7 & 1.64 & 0.122 & \\
\hline 152435.839 & +274221.72 & $\mathrm{gO}$ & 16.67 & 17.96 & 1.29 & 0.12 & 0.10 & 4.40 & 4.50 & -20.1 & 29.7 & 1.16 & 0.068 & \\
\hline 152731.736 & +384308.03 & $\mathrm{gE}$ & 17.00 & 18.02 & 1.02 & 0.05 & 0.18 & 1.43 & 1.26 & -24.1 & 30.7 & 0.63 & 0.400 & \\
\hline
\end{tabular}


TABLE 3-Continued

\begin{tabular}{|c|c|c|c|c|c|c|c|c|c|c|c|c|c|c|}
\hline $\begin{array}{l}\text { RA } \\
(1)\end{array}$ & $\begin{array}{l}\text { Dec } \\
(2)\end{array}$ & $\begin{array}{l}\mathrm{Cl} \\
(3)\end{array}$ & $\begin{array}{c}E \\
(4)\end{array}$ & $\begin{array}{c}\mathrm{O} \\
(5)\end{array}$ & $\begin{array}{l}\text { O-E } \\
(6)\end{array}$ & $\begin{array}{c}\mathrm{A}(\mathrm{E}) \\
(7)\end{array}$ & $\begin{array}{c}\text { Sep } \\
(8)\end{array}$ & $\begin{array}{l}S_{p} \\
(9)\end{array}$ & $\begin{array}{c}S_{i} \\
(10)\end{array}$ & $\begin{array}{c}M_{B} \\
(11)\end{array}$ & $\begin{array}{c}\log L_{R} \\
\text { (12) }\end{array}$ & $\begin{array}{c}\log R^{*} \\
(13)\end{array}$ & $\begin{array}{c}\mathrm{Z} \\
(14)\end{array}$ & $\begin{array}{c}\text { Notes } \\
(15)\end{array}$ \\
\hline 153838.490 & +365729.89 & $\mathrm{gO}$ & 8.04 & 9.97 & 1.92 & 0.05 & 0.63 & 2.29 & 2.85 & -25.3 & 28.4 & -2.22 & 0.019 & $\mathrm{I}, \mathrm{B}=14.7$ \\
\hline 154247.701 & +300512.32 & $*$ & 17.74 & 18.99 & 1.25 & 0.07 & 0.30 & 5.23 & 5.19 & -20.5 & 30.3 & 1.63 & 0.127 & \\
\hline 154458.493 & +230810.36 & $*$ & 16.81 & 17.55 & 0.74 & 0.14 & 0.28 & 20.47 & 21.10 & -24.3 & 31.8 & 1.62 & 0.370 & \\
\hline 155059.392 & +395030.04 & $\mathrm{gE}$ & 16.75 & 17.96 & 1.20 & 0.04 & 0.66 & 10.70 & 10.64 & -23.8 & 31.5 & 1.49 & 0.350 & \\
\hline 155511.010 & +270056.11 & $\mathrm{gO}$ & 17.24 & 18.91 & 1.67 & 0.11 & 0.27 & 1.62 & 1.04 & -19.6 & 29.4 & 1.10 & 0.083 & \\
\hline 155522.049 & +281323.35 & $\mathrm{gE}$ & 16.49 & 18.31 & 1.82 & 0.11 & 0.28 & 14.42 & 14.83 & -21.5 & 30.9 & 1.81 & 0.149 & \\
\hline 160046.973 & +403422.96 & $\mathrm{gE}$ & 16.91 & 18.80 & 1.89 & 0.04 & 0.27 & 2.72 & 2.39 & -19.4 & 29.5 & 1.28 & 0.073 & I \\
\hline 160740.880 & +342715.15 & $\mathrm{gO}$ & 15.99 & 17.82 & 1.84 & 0.05 & 0.25 & 1.19 & 0.87 & -20.6 & 29.2 & 0.53 & 0.079 & \\
\hline 160920.120 & +224157.41 & $\mathrm{gO}$ & 17.10 & 18.75 & 1.65 & 0.22 & 0.77 & 1.57 & 3.50 & -22.8 & 30.9 & 1.33 & 0.320 & \\
\hline 161213.748 & +315648.74 & $\mathrm{gO}$ & 16.06 & 17.53 & 1.47 & 0.07 & 0.97 & 6.24 & 7.04 & -22.8 & 30.7 & 1.17 & 0.185 & I \\
\hline 161336.819 & +233949.41 & $*$ & 17.15 & 17.77 & 0.63 & 0.27 & 0.16 & 3.64 & 3.21 & -22.1 & 30.3 & 0.98 & 0.149 & \\
\hline 162726.656 & +3508 15.82 & $\mathrm{gE}$ & 17.65 & 19.04 & 1.39 & 0.06 & 0.07 & 1.33 & 0.80 & -21.3 & 30.0 & 1.04 & 0.190 & \\
\hline 162910.989 & +285719.50 & $\mathrm{gO}$ & 17.06 & 18.97 & 1.90 & 0.06 & 0.12 & 1.13 & 0.64 & -20.9 & 29.8 & 0.95 & 0.151 & \\
\hline 163929.633 & +274223.36 & $\mathrm{gO}$ & 16.62 & 18.49 & 1.87 & 0.18 & 0.26 & 3.53 & 4.08 & -21.1 & 30.2 & 1.32 & 0.133 & I \\
\hline 164541.190 & +220000.24 & $\mathrm{gE}$ & 17.15 & 18.58 & 1.44 & 0.15 & 0.77 & 1.05 & 2.78 & -21.3 & 30.2 & 1.19 & 0.153 & I \\
\hline 164559.357 & +313641.99 & $\mathrm{gE}$ & 16.92 & 18.48 & 1.55 & 0.08 & 0.31 & 1.36 & 1.95 & -22.0 & 30.2 & 0.98 & 0.199 & \\
\hline 164927.663 & +374632.54 & $\mathrm{gE}$ & 16.68 & 17.98 & 1.29 & 0.07 & 0.56 & 1.10 & 0.98 & -22.1 & 29.8 & 0.54 & 0.167 & \\
\hline 165632.072 & +361934.92 & $\mathrm{gE}$ & 17.20 & 19.15 & 1.95 & 0.04 & 0.42 & 1.37 & 1.81 & -20.7 & 30.0 & 1.23 & 0.150 & \\
\hline 165642.119 & +363048.53 & $\mathrm{gE}$ & 16.93 & 18.62 & 1.69 & 0.04 & 0.59 & 1.39 & 1.46 & -21.1 & 29.8 & 0.93 & 0.140 & \\
\hline 165649.013 & +223526.29 & $\mathrm{gE}$ & 17.53 & 19.38 & 1.85 & 0.20 & 0.84 & 1.05 & 1.50 & -20.8 & 30.0 & 1.24 & 0.178 & \\
\hline 165908.574 & +285931.01 & $\mathrm{gO}$ & 9.19 & 9.15 & -0.04 & 0.11 & 1.18 & 3.54 & 6.07 & -27.3 & 29.1 & -2.22 & 0.032 & $\mathrm{o}, \mathrm{I}, \mathrm{B}=15.2$ \\
\hline 170828.791 & +283305.66 & $\mathrm{gE}$ & 16.28 & 18.06 & 1.77 & 0.19 & 0.51 & 1.77 & 1.41 & -20.6 & 29.5 & 0.80 & 0.087 & I \\
\hline 171322.635 & +325628.10 & $\mathrm{gE}$ & 15.21 & 16.50 & 1.28 & 0.14 & 0.16 & 34.97 & 38.62 & -22.5 & 31.0 & 1.51 & 0.101 & $\mathrm{X}$ \\
\hline 172029.759 & +395555.95 & $\mathrm{gO}$ & 16.84 & 18.19 & 1.35 & 0.10 & 0.82 & 2.03 & 2.33 & -22.2 & 30.3 & 0.95 & 0.190 & \\
\hline 172527.267 & +372631.04 & $\mathrm{gE}$ & 16.89 & 18.74 & 1.85 & 0.11 & 0.28 & 1.10 & 1.04 & -18.1 & 28.6 & 0.87 & 0.039 & \\
\hline
\end{tabular}

Note.-Table columns are same as Table 2. 
TABLE 4

BL LACERTAE OBJeCtS IN FBQS CANDIDATE List

\begin{tabular}{|c|c|c|c|c|c|c|c|c|c|c|c|c|c|c|}
\hline $\begin{array}{l}\text { RA } \\
\text { (1) }\end{array}$ & $\begin{array}{c}\text { Dec } \\
\text { (2) }\end{array}$ & $\begin{array}{l}\mathrm{Cl} \\
(3)\end{array}$ & $\begin{array}{c}E \\
(4)\end{array}$ & $\begin{array}{c}\mathrm{O} \\
(5)\end{array}$ & $\begin{array}{l}\text { O-E } \\
(6)\end{array}$ & $\begin{array}{c}\mathrm{A}(\mathrm{E}) \\
(7)\end{array}$ & $\begin{array}{l}\text { Sep } \\
(8)\end{array}$ & $\begin{array}{l}S_{p} \\
(9)\end{array}$ & $\begin{array}{c}S_{i} \\
(10)\end{array}$ & $\begin{array}{c}M_{B} \\
(11)\end{array}$ & $\begin{array}{c}\log L_{R} \\
\text { (12) }\end{array}$ & $\begin{array}{c}\log R^{*} \\
\text { (13) }\end{array}$ & $\begin{array}{c}\mathrm{Z} \\
(14)\end{array}$ & $\begin{array}{l}\text { Notes } \\
\text { (15) }\end{array}$ \\
\hline 072442.742 & +262130.60 & $\mathrm{gE}$ & 16.74 & 17.64 & 0.89 & 0.22 & 0.54 & 1.24 & 1.15 & $\ldots$ & $\ldots$ & 0.49 & 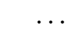 & ? \\
\hline 073026.050 & 330722.71 & $*$ & 17.14 & 17.38 & 0.24 & 0.17 & 0.42 & 9.87 & 9.79 & $\ldots$ & $\ldots$ & 1.29 & $\ldots$ & $\mathrm{X}$ \\
\hline 073837.806 & +313931.25 & $\mathrm{gE}$ & 16.88 & 18.12 & 1.25 & 0.13 & 1.12 & 6.40 & 6.97 & $\ldots$ & $\ldots$ & 1.44 & $\ldots$ & ? \\
\hline 074118.793 & +225340.19 & $\mathrm{gO}$ & 16.34 & 17.21 & 0.88 & 0.13 & 0.96 & 1.29 & 1.50 & $\ldots$ & $\ldots$ & 0.41 & $\ldots$ & ?,I \\
\hline 081421.287 & +294021.07 & * & 17.37 & 17.98 & 0.61 & 0.10 & 0.44 & 26.42 & 27.34 & $\ldots$ & $\ldots$ & 1.97 & $\ldots$ & o \\
\hline $08 \quad 1827.347$ & +281402.68 & $\mathrm{gE}$ & 17.16 & 18.11 & 0.95 & 0.07 & 0.22 & 80.90 & 84.12 & $\ldots$ & $\ldots$ & 2.51 & $\ldots$ & \\
\hline 081925.891 & +403743.88 & $\mathrm{gE}$ & 17.74 & 19.33 & 1.58 & 0.14 & 0.08 & 77.37 & 79.55 & $\ldots$ & $\ldots$ & 2.98 & 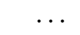 & ? \\
\hline 082020.203 & +364004.81 & * & 17.75 & 18.15 & 0.40 & 0.14 & 0.61 & 96.52 & 102.07 & $\ldots$ & $\cdots$ & 2.61 & $\ldots$ & \\
\hline 083353.885 & +422401.79 & $*$ & 17.08 & 18.37 & 1.30 & 0.07 & 0.16 & 229.81 & 243.78 & $\ldots$ & $\cdots$ & 3.08 & $\ldots$ & \\
\hline 084702.499 & +41 1757.73 & $\mathrm{gO}$ & 16.59 & 17.97 & 1.38 & 0.07 & 0.38 & 1.03 & 0.77 & -21.5 & 29.6 & 0.52 & 0.129 & $\mathrm{Br}_{4000}=18 \%$ \\
\hline 091037.060 & +332924.54 & $\mathrm{gE}$ & 16.44 & 16.56 & 0.12 & 0.06 & 0.53 & 102.94 & 106.23 & $\cdots$ & $\cdots$ & 2.00 & $\ldots$ & $\mathrm{X}$ \\
\hline 091052.033 & +390202.05 & $\mathrm{gE}$ & 16.71 & 17.78 & 1.06 & 0.05 & 0.26 & 7.25 & 8.27 & $\ldots$ & $\ldots$ & 1.37 & $\ldots$ & \\
\hline 091552.398 & +293324.14 & * & 15.35 & 15.83 & 0.47 & 0.07 & 0.19 & 324.36 & 356.43 & $\ldots$ & $\ldots$ & 2.23 & 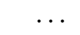 & $\mathrm{o}, \mathrm{X}$ \\
\hline 101244.288 & +422957.01 & $\mathrm{gE}$ & 16.77 & 18.28 & 1.51 & 0.04 & 0.11 & 69.90 & 79.90 & $\ldots$ & $\ldots$ & 2.56 & $\ldots$ & $o, X$ \\
\hline 101616.822 & +4108 12.24 & $\mathrm{gE}$ & 17.32 & 18.99 & 1.67 & 0.05 & 0.15 & 14.98 & 16.57 & -22.3 & 31.5 & 2.10 & 0.281 & $\mathrm{X}, \mathrm{Br}$ \\
\hline 103240.737 & +373827.05 & * & 16.77 & 17.35 & 0.58 & 0.05 & 1.03 & 72.79 & 86.28 & $\ldots$ & $\ldots$ & 2.22 & & \\
\hline 104309.033 & +240835.46 & $*$ & 16.30 & 17.69 & 1.40 & 0.08 & 0.10 & 359.68 & 369.54 & -25.2 & 33.4 & 2.89 & 0.560 & $\mathrm{X}, \mathrm{W}_{\lambda}=5 \mathrm{~A}$ \\
\hline 105431.897 & +385521.71 & $*$ & 16.64 & 16.93 & 0.29 & 0.05 & 0.21 & 72.06 & 73.07 & -28.1 & 33.5 & 1.79 & 1.363 & $\mathrm{X}, \mathrm{W}_{\lambda}=5 \mathrm{~A}$ \\
\hline 105723.103 & +230318.87 & $*$ & 17.74 & 19.26 & 1.52 & 0.05 & 0.41 & 6.28 & 6.73 & -22.7 & 31.3 & 1.80 & 0.379 & $\mathrm{X}, \mathrm{Br}_{4000}=4 \%$ \\
\hline 105829.896 & +281746.43 & $*$ & 17.39 & 18.26 & 0.87 & 0.06 & 0.34 & 77.99 & 91.04 & $\ldots$ & $\ldots$ & 2.61 & $\ldots$ & ? \\
\hline 110021.083 & +401927.68 & $*$ & 17.44 & 18.16 & 0.72 & 0.02 & 0.31 & 14.88 & 15.47 & .. & $\ldots$ & 1.80 & & $\mathrm{o}, \mathrm{X}$ \\
\hline 110427.324 & +381231.60 & $\mathrm{gO}$ & 9.94 & 11.73 & 1.79 & 0.04 & 1.13 & 557.26 & 573.79 & -24.6 & 31.1 & 0.79 & 0.031 & $\mathrm{o}, \mathrm{X}$, \\
\hline 110916.165 & +241120.13 & * & 16.91 & 17.76 & 0.86 & 0.05 & 0.26 & 25.63 & 31.02 & $\ldots$ & $\ldots$ & 1.94 & $\ldots$ & o \\
\hline 111056.838 & +353907.21 & $*$ & 17.43 & 18.07 & 0.64 & 0.05 & 0.24 & 7.52 & 6.85 & $\ldots$ & $\ldots$ & 1.45 & $\ldots$ & o \\
\hline 111740.407 & +25484 & $*$ & 17.16 & 17.92 & 0.77 & 0.05 & 0.33 & 9.92 & 10.05 & -23.9 & 31.5 & 1.45 & 0.360 & $\mathrm{X}, \mathrm{Br}$ \\
\hline 112048.064 & +421212.41 & $*$ & 16.70 & 17.08 & 0.38 & 0.05 & 0.24 & 24.63 & 25.09 & -22.3 & 30.9 & 1.55 & 0.124 & $o, X, z ?$ \\
\hline 112950.143 & +265253.27 & $*$ & 16.19 & 16.81 & 0.62 & 0.05 & 0.74 & 1.58 & 1.81 & $\ldots$ & $\ldots$ & 0.33 & $\ldots$ & o \\
\hline 113650.128 & +255052.45 & $\mathrm{gE}$ & 16.20 & 17.48 & 1.29 & 0.06 & 0.41 & 13.94 & 13.26 & -22.4 & 30.9 & 1.45 & 0.155 & $\mathrm{X}, \mathrm{Br}_{4000}=2$ \\
\hline 115019.220 & +241753.73 & $*$ & 16.72 & 17.57 & 0.85 & 0.07 & 0.26 & 721.89 & 751.10 & $\ldots$ & $\ldots$ & 3.25 & . & \\
\hline 115210.708 & +283721.22 & $*$ & 17.19 & 18.66 & 1.46 & 0.06 & 0.08 & 22.68 & 23.10 & $\ldots$ & $\ldots$ & 2.17 & $\ldots$ & \\
\hline 115342.906 & +38 2305.92 & $\mathrm{gE}$ & 17.67 & 18.36 & 0.69 & 0.05 & 0.08 & 117.11 & 123.84 & $\ldots$ & $\ldots$ & 2.78 & ... & ? \\
\hline 120922.798 & +411941.45 & * & 16.77 & 17.47 & 0.70 & 0.04 & 0.16 & 397.21 & 398.39 & $\ldots$ & $\ldots$ & 2.93 & $\ldots$ & $\mathrm{o}, \mathrm{X}$ \\
\hline 121752.085 & +300700.46 & $\mathrm{gE}$ & 13.86 & 15.40 & 1.54 & 0.06 & 0.31 & 375.97 & 406.29 & -25.5 & 32.7 & 2.07 & 0.237 & $o, X$ \\
\hline 122008.319 & +343121.55 & * & 16.86 & 17.51 & 0.65 & 0.04 & 0.42 & 251.86 & 256.45 & .. & $\ldots$ & 2.76 & & o \\
\hline 122121.941 & +30 1037.10 & $\mathrm{gE}$ & 15.52 & 16.58 & 1.06 & 0.06 & 0.11 & 60.66 & 63.03 & -23.7 & 31.7 & 1.74 & 0.182 & $\mathrm{o}, \mathrm{X}, \mathrm{I}$ \\
\hline 122424.179 & +243623.52 & * & 16.73 & 17.38 & 0.65 & 0.06 & 0.05 & 21.93 & 23.40 & -23.3 & 31.4 & 1.62 & 0.218 & 0 \\
\hline 122604.124 & +260428.01 & $*$ & 17.23 & 18.37 & 1.14 & 0.05 & 0.38 & 9.64 & 10.84 & -21.8 & 30.9 & 1.69 & 0.176 & $?, \mathrm{X}, \mathrm{Br}_{4000}=2$ \\
\hline 123014.099 & +251807.13 & $*$ & 15.97 & 16.87 & 0.90 & 0.05 & 0.32 & 225.99 & 231.69 & $\ldots$ & $\ldots$ & 2.46 & $\ldots$ & $o, X$ \\
\hline 123623.025 & +390001.09 & $*$ & 17.55 & 18.64 & 1.08 & 0.04 & 0.52 & 34.94 & 36.54 & $\ldots$ & $\ldots$ & 2.36 & $\ldots$ & \\
\hline 124312.695 & +362743.91 & $*$ & 16.26 & 16.77 & 0.51 & 0.03 & 0.49 & 113.31 & 140.99 & $\ldots$ & $\ldots$ & 2.20 & 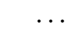 & $\mathrm{X}$ \\
\hline 125219.472 & +26405 & $*$ & 17.49 & 18.07 & 0.58 & 0.03 & 0.28 & 1.56 & 1.38 & $\ldots$ & $\ldots$ & 0.76 & $\ldots$ & ? \\
\hline 125716.578 & +364715.18 & $*$ & 16.35 & 17.59 & 1.24 & 0.04 & 0.61 & 65.93 & 73.86 & -23.7 & 32.1 & 2.20 & 0.280 & $\mathrm{o}, X$ \\
\hline 130145.674 & +40 5624.78 & $*$ & 17.77 & 18.68 & 0.91 & 0.05 & 0.35 & 25.53 & 26.59 & $\ldots$ & $\ldots$ & 2.24 & $\ldots$ & $\mathrm{X}$ \\
\hline 131443.804 & +234826.82 & $*$ & 15.68 & 16.70 & 1.02 & 0.03 & 0.08 & 168.97 & 174.76 & $\ldots$ & $\ldots$ & 2.26 & ... & $X$ \\
\hline 135426.699 & +370654.75 & $\mathrm{gE}$ & 17.68 & 18.56 & 0.89 & 0.03 & 0.34 & 19.07 & 20.00 & & $\cdots$ & 2.07 & & \\
\hline 141409.281 & +343057.58 & $\mathrm{gE}$ & 17.63 & 19.42 & 1.79 & 0.04 & 0.27 & 3.39 & 3.34 & -21.8 & 30.8 & 1.59 & 0.275 & $\mathrm{Br}_{4000}=21 \%$ \\
\hline 142607.716 & +340426.29 & $*$ & 16.94 & 17.46 & 0.52 & 0.04 & 0.18 & 33.51 & 35.70 & $\ldots$ & $\ldots$ & 1.88 & $\ldots$ & \\
\hline 142700.414 & +234800.09 & $*$ & 14.79 & 15.46 & 0.67 & 0.16 & 0.46 & 288.51 & 413.91 & & $\ldots$ & 2.15 & & $\mathrm{o}, \mathrm{X}$ \\
\hline 143040.595 & +364904.14 & $*$ & 17.16 & 17.66 & 0.50 & 0.02 & 0.20 & 160.06 & 162.01 & -25.2 & 33.1 & 2.52 & 0.564 & $\mathrm{~W}_{\lambda}=2 \mathrm{~A}, \mathrm{Br}_{40}$ \\
\hline 143917.498 & +393242.86 & $\mathrm{gE}$ & 16.51 & 18.39 & 1.88 & 0.03 & 0.29 & 42.35 & 44.12 & $\ldots$ & $\ldots$ & 2.34 & $\ldots$ & $\mathrm{o}, \hat{\mathrm{X}}$ \\
\hline 144800.578 & +360831.67 & * & 16.36 & 16.74 & 0.38 & 0.03 & 0.94 & 29.65 & 30.92 & $\ldots$ & $\ldots$ & 1.53 & 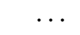 & $o, X$ \\
\hline 101.836 & +223806.52 & $*$ & 15.19 & 16.32 & 1.13 & 0.13 & 0.37 & 28.74 & 29.95 & -24.5 & 31.6 & 1.30 & 0.235 & 0,2 \\
\hline 150208.291 & +252844.91 & $*$ & 16.68 & 18.14 & 1.46 & 0.09 & 0.81 & 1.09 & 4.06 & -20.6 & 29.9 & 1.19 & 0.090 & ? \\
\hline 150233.971 & +335055.84 & $\mathrm{gE}$ & 17.25 & 18.24 & 0.99 & 0.04 & 0.64 & 78.32 & 80.99 & & $\cdots$ & 2.55 & & \\
\hline 151556.188 & +242620.28 & $\mathrm{gE}$ & 16.87 & 18.83 & 1.96 & 0.13 & 0.2 & 23.68 & 30.07 & -22.0 & 31.5 & 2.31 & 0.228 & $\mathrm{Br}_{4000}=17 \%$ \\
\hline 153044.062 & +231013.50 & * & 17.13 & 17.32 & 0.19 & 0.15 & 0.28 & 5.13 & 4.55 & $\ldots$ & $\ldots$ & 0.98 & $\ldots$ & \\
\hline 153324.253 & +341640.24 & $*$ & 17.00 & 17.64 & 0.64 & 0.06 & 0.22 & 27.14 & 27.27 & . & $\ldots$ & 1.84 & . & $\mathrm{o}, \mathrm{X}$ \\
\hline 153447.205 & +37 1554.81 & $\mathrm{gE}$ & 16.24 & 18.18 & 1.94 & 0.05 & 0.15 & 21.75 & 22.14 & -21.5 & 31.0 & 1.93 & 0.138 & $\mathrm{Br}_{4000}=12 \%, \mathrm{~W}_{\lambda}$ \\
\hline 153529.052 & +392246.72 & $\mathrm{gE}$ & 16.73 & 18.46 & 1.73 & 0.05 & 0.27 & 19.20 & 20.14 & -22.6 & 31.5 & 1.98 & 0.257 & $00=9 \%$ \\
\hline 160218.089 & +305109.30 & $*$ & 17.74 & 18.78 & 1.04 & 0.07 & 0.63 & 54.12 & 59.92 & $\ldots$ & $\ldots$ & 2.63 & $\ldots$ & $\mathrm{O}(A$ \\
\hline 161706.360 & +410646.97 & $\mathrm{gE}$ & 16.41 & 18.06 & 1.65 & 0.02 & 0.76 & 55.61 & 63.88 & -23.1 & 32.0 & 2.32 & 0.267 & $\mathrm{X}, \mathrm{B}_{1}$ \\
\hline 164557.715 & +294730.20 & * & 17.80 & 18.03 & 0.23 & 0.10 & 0.49 & 4.59 & 4.55 & $\ldots$ & $\ldots$ & 1.22 & $\ldots$ & $?$ \\
\hline 165109.196 & +421253.40 & $*$ & 17.64 & 18.08 & 0.44 & 0.07 & 0.11 & 16.33 & 17.20 & $\ldots$ & $\ldots$ & 1.81 & $\ldots$ & \\
\hline 165249.928 & +40 2309.93 & $*$ & 16.60 & 17.13 & 0.53 & 0.05 & 0.32 & 16.82 & 17.66 & $\ldots$ & $\ldots$ & 1.44 & 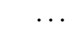 & o \\
\hline 170124.628 & +395437.08 & $*$ & 16.73 & 17.77 & 1.04 & 0.07 & 0.47 & 250.98 & 254.93 & $\ldots$ & $\ldots$ & 2.86 & $\ldots$ & \\
\hline 170209.640 & +264314.72 & $*$ & 17.53 & 18.28 & 0.75 & 0.14 & 0.49 & 50.48 & 52.86 & $\ldots$ & $\ldots$ & 2.38 & $\ldots$ & \\
\hline 170238.546 & +311543.50 & $*$ & 17.58 & 17.97 & 0.39 & 0.09 & 0.09 & 5.54 & 5.81 & $\ldots$ & $\ldots$ & 1.30 & 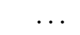 & \\
\hline 171248.706 & +293117.47 & $*$ & 17.80 & 18.24 & 0.44 & 0.15 & 0.77 & 11.54 & 13.39 & $\ldots$ & $\ldots$ & 1.77 & $\ldots$ & 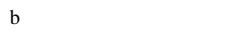 \\
\hline
\end{tabular}


TABLE 5

H II Region Galaxies in FBQS Candidate List

\begin{tabular}{|c|c|c|c|c|c|c|c|c|c|c|c|c|c|c|}
\hline $\begin{array}{l}\text { RA } \\
\text { (1) }\end{array}$ & $\begin{array}{l}\text { Dec } \\
(2)\end{array}$ & $\begin{array}{l}\mathrm{Cl} \\
\text { (3) }\end{array}$ & $\begin{array}{l}E \\
(4)\end{array}$ & $\begin{array}{c}\mathrm{O} \\
(5)\end{array}$ & $\begin{array}{l}\text { O-E } \\
(6)\end{array}$ & $\begin{array}{c}\mathrm{A}(\mathrm{E}) \\
(7)\end{array}$ & $\begin{array}{l}\text { Sep } \\
(8)\end{array}$ & $\begin{array}{l}S_{p} \\
(9)\end{array}$ & $\begin{array}{c}S_{i} \\
(10)\end{array}$ & $\begin{array}{c}M_{B} \\
(11)\end{array}$ & $\begin{array}{c}\log L_{R} \\
\text { (12) }\end{array}$ & $\begin{array}{c}\log R^{*} \\
\text { (13) }\end{array}$ & $\begin{array}{c}\mathrm{Z} \\
(14)\end{array}$ & $\begin{array}{l}\text { Notes } \\
\text { (15) }\end{array}$ \\
\hline 071116.044 & +361507.74 & * & 16.25 & 17.26 & 1.00 & 0.16 & 0.46 & 1.64 & 1.89 & -22.2 & 29.8 & 0.50 & 0.127 & \\
\hline 071533.880 & +330809.97 & $\mathrm{gO}$ & 16.79 & 18.29 & 1.51 & 0.15 & 0.97 & 1.30 & 2.03 & -22.1 & 30.2 & 0.93 & 0.191 & \\
\hline 071653.832 & +4053 37.47 & $\mathrm{gE}$ & 17.26 & 18.49 & 1.23 & 0.15 & 0.25 & 1.05 & 1.34 & -21.1 & 29.7 & 0.84 & 0.132 & \\
\hline 072023.480 & +343532.47 & $\mathrm{gE}$ & 16.42 & 17.92 & 1.50 & 0.14 & 0.55 & 1.15 & 0.96 & -21.9 & 29.8 & 0.54 & 0.148 & \\
\hline 072316.871 & +335957.46 & $\mathrm{gE}$ & 15.30 & 16.39 & 1.08 & 0.15 & 0.51 & 1.20 & 1.55 & -22.0 & 29.3 & 0.07 & 0.077 & \\
\hline 072406.763 & +375531.39 & $\mathrm{gE}$ & 14.38 & 14.87 & 0.49 & 0.14 & 0.56 & 1.97 & 3.75 & -23.0 & 29.5 & -0.15 & 0.061 & \\
\hline 075940.372 & +315030.40 & $\mathrm{gE}$ & 15.59 & 16.28 & 0.69 & 0.13 & 0.17 & 3.72 & 3.83 & -22.4 & 29.8 & 0.42 & 0.087 & I \\
\hline 080148.716 & +260846.18 & $\mathrm{gE}$ & 12.97 & 14.09 & 1.12 & 0.10 & 0.58 & 1.48 & 2.97 & -22.0 & 28.7 & -0.55 & 0.027 & I \\
\hline 081026.038 & +234156.51 & $*$ & 17.55 & 18.04 & 0.49 & 0.13 & 0.61 & 1.39 & 0.90 & -21.5 & 29.7 & 0.68 & 0.133 & $I$ \\
\hline 081246.525 & +262142.05 & $\mathrm{gE}$ & 7.81 & 7.96 & 0.15 & 0.09 & 0.37 & 1.12 & 4.38 & -27.6 & 28.6 & -2.84 & 0.021 & $\mathrm{I}, \mathrm{B}=14.2$ \\
\hline 081450.826 & +253841.42 & $\mathrm{gE}$ & 17.38 & 18.44 & 1.06 & 0.10 & 0.92 & 1.16 & 1.05 & -21.4 & 29.8 & 0.75 & 0.150 & \\
\hline 081911.384 & +402844.63 & $\mathrm{gE}$ & 17.11 & 18.82 & 1.70 & 0.14 & 0.61 & 1.05 & 0.75 & -21.3 & 29.8 & 0.86 & 0.170 & \\
\hline 081923.895 & +261815.34 & $\mathrm{gE}$ & 17.76 & 19.19 & 1.44 & 0.08 & 0.61 & 1.25 & 0.77 & -21.5 & 30.1 & 1.07 & 0.220 & \\
\hline 082045.414 & +422120.40 & $\mathrm{gE}$ & 16.46 & 17.93 & 1.46 & 0.15 & 0.25 & 3.06 & 3.18 & -21.5 & 30.1 & 0.99 & 0.127 & \\
\hline 082302.176 & +364559.03 & $\mathrm{gE}$ & 16.13 & 17.64 & 1.50 & 0.14 & 0.42 & 3.38 & 3.05 & -21.3 & 29.9 & 0.91 & 0.100 & I \\
\hline 082419.454 & +254028.92 & $\mathrm{gE}$ & 13.21 & 14.27 & 1.06 & 0.09 & 0.02 & 1.07 & 1.93 & -21.9 & 28.5 & -0.67 & 0.028 & I \\
\hline 082539.718 & +291133.83 & $\mathrm{gE}$ & 15.94 & 17.37 & 1.43 & 0.11 & 0.74 & 1.71 & 1.17 & -21.4 & 29.5 & 0.50 & 0.092 & I \\
\hline 082547.478 & +234058.50 & $\mathrm{gO}$ & 17.54 & 18.59 & 1.05 & 0.12 & 0.08 & 1.80 & 1.93 & -21.2 & 30.0 & 1.03 & 0.148 & $\mathrm{E}(\mathrm{AI}$ \\
\hline 082924.212 & +370431.64 & $\mathrm{gE}$ & 16.01 & 17.70 & 1.69 & 0.10 & 0.58 & 1.15 & 1.15 & -21.2 & 29.4 & 0.46 & 0.100 & $\mathrm{I}$ \\
\hline 083456.553 & +404830.18 & $*$ & 17.17 & 18.88 & 1.71 & 0.12 & 0.44 & 1.44 & 1.73 & -20.2 & 29.7 & 1.11 & 0.108 & \\
\hline 083559.713 & +243526.87 & $*$ & 16.01 & 16.83 & 0.81 & 0.09 & 0.06 & 1.72 & 2.47 & -21.4 & 29.5 & 0.45 & 0.072 & 1 \\
\hline 083602.634 & +242331.71 & $\mathrm{gE}$ & 15.68 & 16.28 & 0.60 & 0.09 & 0.50 & 1.37 & 1.53 & -22.6 & 29.5 & 0.02 & 0.096 & \\
\hline 083752.477 & +404729.46 & $*$ & 17.80 & 19.27 & 1.46 & 0.11 & 0.18 & 1.23 & 1.10 & -21.6 & 30.2 & 1.09 & 0.240 & \\
\hline 084527.390 & +342508.77 & $\mathrm{gE}$ & 10.42 & 10.66 & 0.24 & 0.08 & 0.94 & 1.84 & 3.98 & -25.3 & 28.8 & -1.80 & 0.026 & $\mathrm{I}, \mathrm{B}=14.7$ \\
\hline 085056.862 & +291200.55 & $\mathrm{gO}$ & 12.84 & 13.68 & 0.84 & 0.10 & 0.45 & 1.43 & 3.32 & -22.3 & 28.7 & -0.67 & 0.026 & \\
\hline 085318.995 & +424042.56 & $\mathrm{gO}$ & 11.91 & 12.87 & 0.96 & 0.08 & 0.85 & 1.19 & 4.00 & -24.5 & 29.3 & -0.92 & 0.048 & I \\
\hline 085527.072 & +373922.83 & $\mathrm{gO}$ & 16.98 & 18.57 & 1.59 & 0.09 & 0.34 & 1.16 & 1.01 & -21.5 & 29.9 & 0.80 & 0.167 & \\
\hline 085533.219 & +311242.29 & $\mathrm{gO}$ & 11.63 & 12.80 & 1.17 & 0.07 & 0.34 & 1.66 & 3.20 & -25.3 & 29. & -1.05 & 0.069 & $\mathrm{I}, \mathrm{B}=$ \\
\hline 085540.642 & +254356.30 & $\mathrm{gO}$ & 17.26 & 18.71 & 1.45 & 0.11 & 0.60 & 1.21 & 1.02 & -19.7 & 29.2 & 0.89 & 0.078 & \\
\hline 085940.716 & +404558.50 & $*$ & 17.63 & 18.28 & 0.66 & 0.06 & 0.24 & 1.13 & 0.81 & -21.0 & 29.5 & 0.69 & 0.117 & \\
\hline 090205.530 & +360742.20 & $\mathrm{gE}$ & 17.42 & 18.58 & 1.16 & 0.08 & 0.17 & 1.05 & 0.87 & -22.0 & 30.0 & 0.76 & 0.210 & \\
\hline 091408.992 & +385908.98 & $\mathrm{gE}$ & 16.62 & 17.52 & 0.90 & 0.05 & 0.25 & 3.86 & 3.91 & -22.5 & 30.4 & 0.91 & 0.162 & I \\
\hline 091749.545 & +364911.16 & $\mathrm{gO}$ & 17.04 & 18.52 & 1.48 & 0.04 & 0.35 & 1.98 & 1.16 & -20.2 & 29.6 & 1.03 & 0.089 & I \\
\hline 092253.016 & +395711.93 & $\mathrm{gE}$ & 17.43 & 18.10 & 0.66 & 0.03 & 0.36 & 3.90 & 5.01 & -23.2 & 31.0 & 1.23 & 0.288 & \\
\hline 092453.202 & +410336.93 & $\mathrm{gE}$ & 8.09 & 9.77 & 1.68 & 0.05 & 0.88 & 4.63 & 6.40 & -24.9 & 28.5 & -1.94 & 0.014 & \\
\hline 092455.002 & +341535.77 & $\mathrm{gE}$ & 16.69 & 17.55 & 0.87 & 0.05 & 0.10 & 1.83 & 1.33 & -20.5 & 29.3 & 0.61 & 0.068 & I \\
\hline 092608.227 & +345343.56 & $\mathrm{gO}$ & 12.48 & 12.87 & 0.38 & 0.06 & 0.47 & 3.06 & 4.28 & -22.1 & 28.4 & -0.88 & 0.016 & $\mathrm{I}, \mathrm{B}=16.5$ \\
\hline 092625.308 & +352755.42 & $\mathrm{gE}$ & 15.98 & 16.72 & 0.73 & 0.05 & 0.17 & 1.86 & 1.80 & -23.0 & 29.9 & 0.27 & 0.142 & \\
\hline 092746.008 & +235559.51 & $\mathrm{gO}$ & 10.73 & 9.52 & -1.21 & 0.09 & 1.06 & 2.06 & 4.13 & -26.9 & 29.0 & -2.24 & 0.032 & $\mathrm{I}, \mathrm{B}=15.4$ \\
\hline 093011.081 & +343951.98 & $\mathrm{gO}$ & 11.66 & 12.36 & 0.70 & 0.04 & 0.13 & 5.71 & 11.21 & -23.4 & 29.1 & -0.67 & 0.023 & $=15.5$ \\
\hline 093737.670 & +231426.73 & $*$ & 10.43 & 8.87 & -1.56 & 0.09 & 1.05 & 1.50 & 5.10 & -27.0 & 28.9 & -2.41 & 0.025 & $\mathrm{I}, \mathrm{B}=14.9$ \\
\hline 094036.384 & +414057.37 & $\mathrm{gE}$ & 17.13 & 18.93 & 1.80 & 0.03 & 0.50 & 1.17 & 0.75 & -20.4 & 29.6 & 0.96 & 0.121 & \\
\hline 094137.718 & +381945.44 & $*$ & 17.47 & 19.38 & 1.91 & 0.05 & 0.23 & 2.64 & 1.90 & -21.2 & 30.4 & 1.48 & 0.208 & I \\
\hline 094552.257 & +344108.34 & $*$ & 9.62 & 10.31 & 0.69 & 0.03 & 0.56 & 1.67 & 8.17 & -25.1 & 28 & -1.62 & 0.020 & $\mathrm{I}, \mathrm{B}$ \\
\hline 094644.582 & +291335.64 & $*$ & 16.49 & 17.84 & 1.35 & 0.05 & 0.16 & 1.21 & 0.93 & -21.9 & 29. & 0.53 & 0.140 & $I$ \\
\hline 095143.767 & +361610.72 & $\mathrm{gE}$ & 16.83 & 18.49 & 1.66 & 0.03 & 0.43 & 1.08 & 1.04 & -20.9 & 29.5 & 0.75 & 0.120 & \\
\hline 095642.683 & +362029.04 & $\mathrm{gE}$ & 16.27 & 17.73 & 1.45 & 0.03 & 0.63 & 1.25 & 1.01 & -21.9 & 29.7 & 0.50 & 0.137 & \\
\hline 095813.006 & +342533.07 & $\mathrm{gE}$ & 16.97 & 18.40 & 1.43 & 0.03 & 0.28 & 1.47 & 1.47 & -21.1 & 29 & 0.84 & 0.130 & I \\
\hline 102050.970 & +3306 49.77 & * & 16.24 & 17.38 & 1.13 & 0.07 & 0.34 & 1.86 & 1.56 & -22.1 & 29.8 & 0.54 & 0.126 & $\mathrm{O}(\mathrm{APS}), \mathrm{I}$ \\
\hline 102250.589 & +291102.80 & $\mathrm{gE}$ & 15.95 & 17.57 & 1.61 & 0.08 & 0.18 & 1.80 & 1.36 & -22.0 & 29.9 & 0.60 & 0.134 & \\
\hline 102256.211 & +232150.79 & $\mathrm{gE}$ & 16.44 & 17.94 & 1.50 & 0.05 & 0.28 & 1.22 & 0.87 & -22.8 & 30.1 & 0.56 & 0.226 & I \\
\hline 103209.106 & +340252.52 & $\mathrm{gE}$ & 15.69 & 16.36 & 0.66 & 0.05 & 0.63 & 1.25 & 1.92 & -22.5 & 29. & 0.15 & 0.097 & \\
\hline 103222.731 & +355036.00 & * & 17.51 & 18.72 & 1.20 & 0.06 & 0.25 & 1.17 & 0.89 & -21.3 & 29.9 & 0.87 & 0.165 & \\
\hline 103535.900 & +323254.18 & $\mathrm{gE}$ & 15.28 & 16.16 & 0.88 & 0.05 & 0.50 & 1.11 & 0.83 & -22.3 & 29.2 & -0.16 & 0.080 & I \\
\hline 103540.015 & +355124.16 & . & 17.15 & 18.87 & 1.72 & 0.07 & 0.33 & 1.70 & 1.25 & -21.0 & 30 & 1.09 & 0.154 & \\
\hline 103616.829 & +315732.28 & $\mathrm{gE}$ & 16.46 & 18.44 & 1.98 & 0.06 & 0.11 & 1.64 & 0.93 & -21.7 & 30.0 & 0.90 & 0.170 & I \\
\hline 103642.768 & +262823.59 & $\mathrm{gO}$ & 17.36 & 19.01 & 1.65 & 0.06 & 0.64 & 1.67 & 1.48 & -19.7 & 29.5 & 1.15 & 0.090 & \\
\hline 103646.076 & +243424.76 & $\mathrm{gO}$ & 16.06 & 17.67 & 1.61 & 0.06 & 0.79 & 1.0 & 1.40 & -22.5 & 30 & 0.52 & 0.170 & \\
\hline 103806.537 & +391037.34 & * & 9.65 & 8.95 & -0.70 & 0.07 & 0.72 & 1.39 & 4.61 & -27.4 & 29.0 & -2.42 & 0.031 & $\mathrm{I}, \mathrm{B}=14.9$ \\
\hline 104044.498 & +390428.00 & $*$ & 10.12 & 9.46 & -0.66 & 0.04 & 0.32 & 1.49 & 10.23 & -26.8 & 29.3 & -1.87 & 0.030 & \\
\hline 104109.405 & +344301.54 & $\mathrm{gE}$ & 11.22 & 12.35 & 1.13 & 0.05 & 0.47 & 1.84 & 4.83 & -25.4 & 29 & -1.05 & 0.059 & $\mathrm{~B}-15$ \\
\hline 104131.213 & +371846.48 & $\mathrm{gE}$ & 10.55 & 10.57 & 0.02 & 0.04 & 0.52 & 4.34 & 4.53 & -25.2 & 28.8 & -1.78 & 0.024 & $\mathrm{I}, \mathrm{B}=13.9$ \\
\hline 104413.990 & +224333.56 & $\mathrm{gE}$ & 9.52 & 11.15 & 1.63 & 0.08 & 0.97 & 1.09 & 9.30 & -24.1 & 28.9 & -1.23 & 0.019 & $\mathrm{I}, \mathrm{B}=15.2$ \\
\hline 104844.177 & +260312.28 & $\mathrm{gE}$ & 11.67 & 11.15 & -0.52 & 0.09 & 0.13 & 7.49 & 10.67 & -24.7 & 29.2 & -1.17 & 0.025 & $\mathrm{I}, \mathrm{B}=15.0$ \\
\hline 105137.003 & +420313.46 & $\mathrm{gE}$ & 17.48 & 19.03 & 1.55 & 0.04 & 0.24 & 1.65 & 1.38 & -20.2 & 29.7 & 1.15 & 0.115 & I \\
\hline 110214.046 & +380234.90 & $\mathrm{gE}$ & 15.95 & 17.13 & 1.18 & 0.04 & 0.42 & 9.53 & 9.61 & -22.8 & 30.7 & 1.14 & 0.158 & I \\
\hline 110340.317 & +375545.58 & $*$ & 12.00 & 12.41 & 0.41 & 0.04 & 0.43 & 2.88 & 4.58 & -24.7 & 29.3 & -1.04 & 0.043 & $\mathrm{I}, \mathrm{B}=15.6$ \\
\hline & +342419.76 & $\mathrm{gE}$ & 16.33 & 17.30 & 0.97 & 0.06 & 0.98 & 1.31 & 1.93 & -22.0 & 29.8 & 0.52 & 0.120 & \\
\hline 110601.967 & +400642.29 & $\mathrm{gE}$ & 17.70 & 18.93 & 1.23 & 0.03 & 0.53 & 1.10 & 4.45 & -19.7 & 29.9 & 1.55 & 0.088 & \\
\hline 111002.208 & +313001.88 & * & 16.84 & 17.23 & 0.39 & 0.06 & 0.54 & 1.06 & 1.44 & -22.1 & 29.7 & 0.37 & 0.120 & I \\
\hline 112124.131 & +291046.89 & $*$ & 17.73 & 19.05 & 1.31 & 0.04 & 0.72 & 1.51 & 1.11 & -21.2 & 30.0 & 1.11 & 0.176 & \\
\hline
\end{tabular}


TABLE 5-Continued

\begin{tabular}{|c|c|c|c|c|c|c|c|c|c|c|c|c|c|c|}
\hline $\begin{array}{l}\text { RA } \\
(1)\end{array}$ & $\begin{array}{l}\text { Dec } \\
(2)\end{array}$ & $\begin{array}{l}\mathrm{Cl} \\
(3)\end{array}$ & $\begin{array}{c}E \\
(4)\end{array}$ & $\begin{array}{c}\mathrm{O} \\
(5)\end{array}$ & $\begin{array}{l}\text { O-E } \\
(6)\end{array}$ & $\begin{array}{c}A(E) \\
(7)\end{array}$ & $\begin{array}{c}\text { Sep } \\
(8)\end{array}$ & $\begin{array}{l}S_{p} \\
(9)\end{array}$ & $\begin{array}{c}S_{i} \\
(10)\end{array}$ & $\begin{array}{c}M_{B} \\
(11)\end{array}$ & $\begin{array}{c}\log L_{R} \\
\text { (12) }\end{array}$ & $\begin{array}{c}\log R^{*} \\
(13)\end{array}$ & $\begin{array}{c}\mathrm{Z} \\
(14)\end{array}$ & $\begin{array}{l}\text { Notes } \\
(15)\end{array}$ \\
\hline 8.991 & 170511.45 & $\mathrm{gO}$ & 10.06 & .93 & .88 & 0.07 & 37 & 1.40 & 1.12 & -25.8 & 8.6 & -2.14 & 0.036 & $\mathrm{I}, \mathrm{B}$ \\
\hline .184 & 120904.30 & $\mathrm{gE}$ & 17.46 & 19.16 & 1.71 & 0.04 & 0.92 & & 1.23 & -21.1 & 30.0 & 1.06 & 0.180 & \\
\hline 113243.759 & +340524.36 & $\mathrm{gE}$ & 17.09 & 18.41 & 1.32 & 0.07 & 0.35 & 1.56 & 1.61 & -22.6 & 30.4 & 0.86 & 0.250 & \\
\hline 113243.805 & +415609.53 & $\mathrm{gE}$ & 16.27 & 18.19 & 1.92 & 0.07 & 1.10 & 1.07 & 0.94 & -19.8 & 29.0 & 0.63 & 0.066 & I \\
\hline 114137.986 & +375932.05 & $*$ & 10.19 & 11.40 & 1.22 & 0.06 & 0.82 & 1.70 & 5.90 & -24.2 & 28.8 & -1.33 & 0.022 & $\mathrm{~B}=$ \\
\hline 115316.934 & +232752.15 & $\mathrm{gO}$ & 7.25 & 8.77 & 1.53 & 0.09 & 0.58 & 1.49 & 3.42 & -27.1 & 28.7 & -2.62 & 0.025 & $\mathrm{I}, \mathrm{B}=15.0$ \\
\hline 115741.475 & +321316.54 & $\mathrm{gO}$ & 16.11 & 16.91 & 0.80 & 0.06 & 0.20 & 2.90 & 3.18 & -23.1 & 30.3 & 0.58 & 0.160 & \\
\hline 120200.808 & +323319.60 & $*$ & 17.18 & 18.35 & 1.17 & 0.05 & 0.54 & 2.97 & 2.60 & -21.7 & 30.3 & 1.12 & 0.166 & I \\
\hline 121329.455 & +322331.91 & $\mathrm{gO}$ & 16.89 & 18.35 & 1.46 & 0.04 & 0.66 & 1.37 & 0.88 & -22.0 & 30.0 & 0.78 & 0.190 & \\
\hline 121351.473 & +362231.08 & $*$ & 16.56 & 17.20 & 0.64 & 0.06 & 0.16 & 1.28 & 0.78 & -21.4 & 29.3 & 0.31 & 0.087 & I \\
\hline 122059.798 & +245056.00 & $\mathrm{gE}$ & 14.94 & 15.80 & 0.85 & 0.06 & 0.80 & 1.56 & 1.68 & -22.5 & 29.3 & -0.13 & 0.076 & \\
\hline 122724.582 & +251714.41 & $\mathrm{gE}$ & 14.76 & 15.50 & 0.74 & 0.05 & 0.54 & 1.13 & 1.36 & -22.4 & 29.1 & -0.34 & 0.063 & I \\
\hline 123330.953 & +315549.28 & $*$ & 17.31 & 18.66 & 1.35 & 0.04 & 0.09 & 1.56 & 1.48 & -21.3 & 30.0 & 0.97 & 0.160 & I \\
\hline 123513.183 & +374838.67 & $\mathrm{gE}$ & 17.45 & 19.38 & 1.93 & 0.04 & 0.60 & 1.20 & 0.71 & -21.3 & 30.1 & 1.13 & 0.213 & I \\
\hline 123650.793 & +382402.97 & $\mathrm{gE}$ & 17.79 & 18.85 & 1.06 & 0.03 & 0.24 & 1.16 & 0.84 & -20.5 & 29.6 & 0.92 & 0.118 & \\
\hline 124707.730 & +370536.89 & $\mathrm{gO}$ & 17.75 & 18.41 & 0.66 & 0.05 & 0.75 & 2.33 & 2.17 & -21.5 & 30.1 & 1.04 & 0.158 & I \\
\hline 124708.484 & +274735.26 & $\mathrm{gO}$ & 9.87 & 10.48 & 0.62 & 0.03 & 0.94 & 1.37 & 5.93 & -25.4 & 28.9 & -1.69 & 0.025 & $\mathrm{I}, \mathrm{B}=16$ \\
\hline 124750.275 & +272210.03 & $\mathrm{gE}$ & 15.16 & 16.11 & 0.95 & 0.04 & 0.06 & 3.05 & 3.45 & -22.6 & 29.8 & 0.31 & 0.092 & I \\
\hline 124857.776 & +345207.85 & $\mathrm{gO}$ & 11.38 & 13.12 & 1.74 & 0.04 & 0.34 & 1.55 & 1.86 & -22.6 & 28.3 & -1.14 & 0.023 & $\mathrm{I}, \mathrm{B}$ \\
\hline 125354.492 & +262436.62 & $\mathrm{gO}$ & 15.66 & 16.56 & 0.90 & 0.03 & 0.90 & 1.47 & 1.22 & -20.6 & 28.8 & 0.13 & 0.045 & I \\
\hline 130330.661 & +320455.65 & $\mathrm{gO}$ & 14.68 & 16.16 & 1.48 & 0.04 & 0.20 & 3.53 & 3.20 & -22.2 & 29.7 & 0.34 & 0.077 & I \\
\hline 130613.146 & +235853.98 & $*$ & 17.14 & 18.74 & 1.60 & 0.06 & 0.24 & 2.46 & 2.59 & -21.4 & 30.2 & 1.22 & 0.169 & \\
\hline 131018.728 & +22433 & $\mathrm{gE}$ & 17.65 & 18.95 & 1.31 & 0.04 & 0.72 & 1.14 & 1.52 & -21.4 & 30.1 & 1.07 & 0.188 & \\
\hline 131503.527 & +243707.92 & $\mathrm{gO}$ & 8.48 & 9.70 & 1.22 & 0.04 & 0.15 & 31.21 & 32.33 & -24.8 & 29.1 & -1.27 & 0.013 & I,B \\
\hline 132048.097 & +364548.67 & $\mathrm{gE}$ & 17.02 & 18.01 & 1.00 & 0.04 & 0.64 & 1.07 & 1.03 & -21.1 & 29.5 & 0.56 & 0.110 & \\
\hline 132155.794 & +260957.64 & $\mathrm{gE}$ & 16.43 & 17.10 & 0.67 & 0.06 & 0.56 & 1.04 & 1.69 & -22.6 & 29.9 & 0.38 & 0.140 & \\
\hline 132254.945 & +425436.32 & $*$ & 15.28 & 16.93 & 1.65 & 0.04 & 0.26 & 1.34 & 1.76 & -21.4 & 29.4 & 0.35 & 0.075 & I \\
\hline 132306.672 & +340026.11 & $\mathrm{gO}$ & 16.91 & 17.52 & 0.61 & 0.03 & & & 0.94 & -22.3 & 29.8 & 0.40 & & \\
\hline 132646.626 & +253354.13 & $\mathrm{gE}$ & 17.36 & 18.37 & 1.01 & 0.03 & 0.39 & 1.09 & 0.91 & -21.3 & 29.7 & 0.70 & 0.137 & \\
\hline 133359.731 & +274402.26 & $\mathrm{gE}$ & 17.53 & 19.10 & 1.57 & 0.03 & 0.65 & 1.11 & 1.24 & -21.3 & 30.0 & 1.04 & 0.190 & \\
\hline 134107.944 & +263048.10 & $\mathrm{gO}$ & 17.74 & 18.64 & 0.90 & 0.03 & 0.79 & 1.56 & 0.96 & -19.5 & 29.2 & 0.98 & 0.070 & $?, \mathrm{o}$ \\
\hline 134235.906 & +394533.23 & $\mathrm{gE}$ & 15.50 & 16.67 & 1.17 & 0.02 & 0.08 & 1.32 & 1.39 & -22.3 & 29.5 & 0.14 & 0.100 & \\
\hline 134545.954 & +234212.42 & $\mathrm{gE}$ & 14.98 & 16.07 & 1.10 & 0.04 & 0.38 & 1.82 & 2.41 & -22.5 & 29.6 & 0.14 & 0.083 & I \\
\hline 134919.256 & +340142.08 & $*$ & 15.85 & 17.50 & 1.65 & 0.06 & 0.79 & 1.35 & 1.29 & -21.4 & 9.5 & 0.45 & 0.096 & I \\
\hline 135401.081 & +333208.79 & $*$ & 16.04 & 17.32 & 1.27 & 0.06 & 0.33 & 1.09 & 1.13 & -21.0 & 29.2 & 0.31 & 0.077 & \\
\hline 135450.750 & +234209.67 & $\mathrm{gE}$ & 16.80 & 18.38 & 1.58 & 0.05 & 0.62 & 1.38 & 2.21 & -19.5 & 29.3 & 1.03 & 0.063 & I \\
\hline 135928.825 & +331916.04 & $*$ & 16.60 & 17.87 & 1.27 & 0.04 & 0.44 & 2.12 & 2.34 & -21.8 & 30.0 & 0.83 & 0.140 & I \\
\hline 140128.976 & +351430.98 & $\mathrm{gE}$ & 17.51 & 19.19 & 1.68 & 0.03 & 1.04 & 1.50 & 1.58 & -21.3 & 30.2 & 1.18 & 0.200 & \\
\hline 140337.679 & +370355.44 & $\mathrm{gE}$ & 17.46 & 19.11 & 1.65 & 0.02 & 0.28 & 2.05 & 1.96 & -21.5 & 30.3 & 1.26 & 0.210 & I \\
\hline 140935.441 & +260621.64 & $\mathrm{gE}$ & 15.98 & 16.37 & 0.39 & 0.04 & 0.88 & 1.43 & 1.25 & -21.8 & 29.2 & 0.03 & 0.069 & \\
\hline 141001.541 & +38 1633.10 & $\mathrm{gE}$ & 17.34 & 18.30 & 0.96 & 0.02 & 0.45 & 1.31 & 0.73 & -21.3 & 29.7 & 0.75 & 0.137 & I \\
\hline 141520.502 & +3431 19.04 & $\mathrm{gE}$ & 13.46 & 14.36 & 0.90 & 0.04 & & & & -24.2 & 30.0 & -0.16 & 0.083 & $\mathrm{I}, \mathrm{I}$ \\
\hline 141622.128 & +230944.44 & $\mathrm{gO}$ & 17.53 & 18.62 & 1.10 & 0.07 & 0.17 & 1.23 & 0.82 & -21.1 & 29.7 & 0.85 & 0.140 & \\
\hline 141758.755 & +262447.40 & $*$ & 8.43 & 7.71 & -0.72 & 0.06 & 0.96 & 1.15 & 6.13 & -26.9 & 28.4 & -2.79 & 0.014 & $\mathrm{I}, \mathrm{B}=14.1$ \\
\hline & +26175 & $*$ & & 10.52 & 1.84 & 0.05 & & & & & & -1.47 & 0.0 & 14.7 \\
\hline 141921.796 & +261817.85 & $\mathrm{gO}$ & 10.52 & 12.07 & 1.55 & 0.05 & 0.84 & 1.07 & 4.33 & -24.6 & 29.1 & -1.20 & 0.036 & $B=15.7$ \\
\hline 142839.563 & +293428.90 & $\mathrm{gO}$ & 17.65 & 18.79 & 1.14 & 0.04 & 0.46 & 2.42 & 1.99 & -21.1 & 30.1 & 1.21 & 0.153 & \\
\hline 42.628 & & $\mathrm{gO}$ & 17.79 & 19.25 & 1.46 & 0.04 & & & 6 & & 9 & & 0.190 & \\
\hline 143010.954 & +230134.99 & $\mathrm{gO}$ & 9.03 & 10.92 & 1.89 & 0.08 & 0.58 & 3.89 & 4.19 & -24.1 & 28.4 & -1.67 & 0.017 & $\mathrm{I}, \mathrm{B}=15.3$ \\
\hline 143020.760 & +254809.51 & $*$ & 17.49 & 18.67 & 1.18 & 0.07 & 0.77 & 1.18 & 1.59 & -22.4 & 30.4 & 0.96 & 0.260 & \\
\hline 143632.072 & +414108.03 & $*$ & 8.51 & 9.59 & 1.08 & 0.04 & 0.41 & 1.94 & 9.18 & -25.6 & & .86 & 0.018 & $\mathrm{I}, \mathrm{B}=14.7$ \\
\hline 143811.056 & +400433.32 & $\mathrm{gE}$ & 17.07 & 18.81 & 1.73 & 0.04 & 0.08 & 1.91 & 1.78 & -22.4 & 30.5 & 1.09 & 0.270 & \\
\hline 144240.807 & +262332.84 & $\mathrm{gO}$ & 15.14 & 16.18 & 1.04 & 0.06 & 0.22 & 3.17 & 3.40 & -22.9 & 30.0 & 0.33 & 0.108 & $\mathrm{X}$ \\
\hline 144828.756 & +343438.96 & $*$ & 17.44 & 19.00 & 1.56 & 0.07 & 0.92 & 2.61 & 2.79 & -21.6 & 30.4 & 1.35 & 0.210 & I \\
\hline 144854.519 & +355824.03 & $\mathrm{gE}$ & 16.58 & 18.44 & 1.86 & 0.04 & 0.45 & 1.43 & 1.11 & -20.5 & 29.5 & 0.85 & 0.100 & I \\
\hline 145838.605 & +382728.54 & $\mathrm{gE}$ & 17.22 & 18.90 & 1.67 & 0.04 & 0.65 & 1.71 & 1.99 & -20.8 & 29.9 & 1.17 & 0.140 & \\
\hline 145924.936 & +290037.15 & $\mathrm{gE}$ & 17.04 & 18.32 & 1.28 & 0.05 & 1.07 & 1.33 & 1.92 & -21.5 & 30.0 & 0.92 & 0.151 & \\
\hline 145927.507 & +253502.33 & $*$ & 8.39 & 9.13 & 0.74 & 0.11 & 0.63 & 1.07 & 7.41 & -27.4 & 29.3 & -2.14 & 0.033 & $\mathrm{I}, \mathrm{B}=15.3$ \\
\hline 145932.851 & +263259.78 & $\mathrm{gO}$ & 15.77 & 16.55 & 0.78 & 0.11 & 0.62 & 1.31 & 1.93 & -22.6 & 29.7 & 0.22 & 0.110 & \\
\hline 150235.768 & +354334.60 & $*$ & 16.84 & 18.08 & 1.23 & 0.04 & 1.03 & 1.02 & 1.38 & -21.3 & 29.7 & 0.69 & 0.120 & \\
\hline 150250.406 & +241929.83 & $\mathrm{gO}$ & 17.06 & 18.57 & 1.51 & 0.14 & 0.59 & 1.74 & 2.37 & -20.6 & 29.8 & 1.12 & 0.110 & I \\
\hline 150354.340 & +313320.74 & $\mathrm{gO}$ & 15.75 & 16.89 & 1.14 & 0.05 & 0.67 & 1.04 & 1.54 & -21.5 & 29.3 & 0.27 & 0.079 & I \\
\hline 150435.875 & +344337.10 & $\mathrm{gO}$ & 16.86 & 17.29 & 0.43 & 0.04 & 0.90 & 1.10 & 2.41 & -21.6 & 29.7 & 0.62 & 0.100 & I \\
\hline 150555.221 & +352441.64 & $\mathrm{gO}$ & 17.24 & 18.35 & 1.11 & 0.04 & 0.80 & 1.08 & 0.87 & -20.4 & 29.3 & 0.70 & 0.092 & \\
\hline 152211.929 & +331354.24 & $\mathrm{gO}$ & 16.49 & 17.84 & 1.35 & 0.06 & 0.45 & 2.57 & 2.95 & -20.7 & 29.7 & 0.93 & 0.082 & \\
\hline 152610.574 & +394332.85 & $\mathrm{gE}$ & 14.98 & 16.08 & 1.10 & 0.06 & 0.05 & 1.19 & 0.99 & -22.4 & 29.2 & -0.17 & 0.081 & I \\
\hline 152801.515 & +285957.72 & $\mathrm{gE}$ & 14.59 & 15.74 & 1.15 & 0.06 & 1.00 & 1.13 & 1.95 & -22.4 & 29.3 & -0.09 & 0.070 & I \\
\hline 152840.942 & +344202.08 & $*$ & 16.56 & 17.75 & 1.19 & 0.07 & 0.54 & 1.50 & 1.09 & -22.2 & 29.9 & 0.59 & 0.156 & I \\
\hline 153341.848 & +260823.95 & $\mathrm{gE}$ & 10.09 & 10.73 & 0.64 & 0.15 & 0.20 & 1.79 & 4.29 & -25.8 & 29.1 & -1.74 & 0.034 & $\mathrm{I}, \mathrm{B}=15.3$ \\
\hline 153755.817 & +232555.36 & $\mathrm{gE}$ & 16.16 & 17.50 & 1.34 & 0.13 & 0.44 & 1.35 & 1.15 & -21.4 & 29.5 & 0.46 & 0.099 & \\
\hline 153841.207 & +292732.42 & $\mathrm{gE}$ & 15.01 & 16.17 & 1.16 & 0.06 & 0.20 & 1.47 & 1.43 & -21.6 & 29.1 & -0.03 & 0.060 & I \\
\hline
\end{tabular}


TABLE 5-Continued

\begin{tabular}{|c|c|c|c|c|c|c|c|c|c|c|c|c|c|c|}
\hline $\begin{array}{l}\text { RA } \\
\text { (1) }\end{array}$ & $\begin{array}{c}\text { Dec } \\
(2)\end{array}$ & $\begin{array}{l}\mathrm{Cl} \\
(3)\end{array}$ & $\begin{array}{c}E \\
(4)\end{array}$ & $\begin{array}{c}\mathrm{O} \\
(5)\end{array}$ & $\begin{array}{c}\text { O-E } \\
(6)\end{array}$ & $\begin{array}{c}A(E) \\
(7)\end{array}$ & $\begin{array}{c}\text { Sep } \\
(8)\end{array}$ & $\begin{array}{l}S_{p} \\
(9)\end{array}$ & $\begin{array}{c}S_{i} \\
(10)\end{array}$ & $\begin{array}{c}M_{B} \\
(11)\end{array}$ & $\begin{array}{c}\log L_{R} \\
\text { (12) }\end{array}$ & $\begin{array}{c}\log R^{*} \\
\text { (13) }\end{array}$ & $\begin{array}{c}\mathrm{Z} \\
(14)\end{array}$ & $\begin{array}{c}\text { Notes } \\
(15)\end{array}$ \\
\hline 39.089 & 223224.84 & $\mathrm{gE}$ & 7.67 & 19.28 & 1.61 & 0.13 & 0.09 & 1.24 & 1.48 & -20.2 & 29.8 & 1.20 & 0.130 & \\
\hline 154212.687 & +361515.15 & $*$ & 17.11 & 19.07 & 1.96 & 0.07 & 0.58 & 1.63 & 1.97 & -21.8 & 30.4 & 1.22 & 0.240 & I \\
\hline 154326.407 & +322859.29 & $\mathrm{gE}$ & 17.13 & 18.39 & 1.26 & 0.08 & 0.59 & 1.84 & 1.67 & -22.1 & 30.2 & 0.92 & 0.200 & I \\
\hline 154543.143 & +225242.47 & $\mathrm{gE}$ & 10.11 & 10.75 & 0.63 & 0.15 & 0.90 & 1.19 & 3.10 & -26.2 & 29.1 & -1.87 & 0.041 & $\mathrm{I}, \mathrm{B}=$ \\
\hline 154607.495 & +282457.02 & $\mathrm{gE}$ & 12.74 & 13.61 & 0.87 & 0.08 & 0.35 & 1.25 & 2.99 & -23.0 & 28.9 & -0.74 & 0.034 & $\mathrm{I}, \mathrm{B}=15.5$ \\
\hline 154636.805 & +385628.82 & $\mathrm{gE}$ & 15.71 & 16.95 & 1.24 & 0.04 & 0.28 & 1.56 & 1.63 & -21.1 & 29.2 & 0.32 & 0.068 & \\
\hline 154652.916 & +263448.07 & $\mathrm{gE}$ & 16.85 & 18.31 & 1.46 & 0.12 & 0.46 & 2.47 & 2.63 & -19.9 & 29.5 & 1.07 & 0.072 & I \\
\hline 154736.342 & +260349.45 & $\mathrm{gE}$ & 8.51 & 9.58 & 1.07 & 0.16 & 0.58 & 2.51 & 5.42 & -26.8 & 29.1 & -2.10 & 0.031 & $\mathrm{I}, \mathrm{B}=14.9$ \\
\hline 154823.287 & +263728.48 & $\mathrm{gE}$ & 15.51 & 16.46 & 0.95 & 0.11 & 0.43 & 1.49 & 1.75 & -22.5 & 29.6 & 0.15 & 0.100 & I \\
\hline 155535.832 & +315257.92 & $*$ & 17.70 & 19.35 & 1.65 & 0.07 & 0.84 & 5.84 & 6.45 & -21.2 & 30.8 & 1.85 & 0.210 & \\
\hline 155536.222 & +312502.88 & $*$ & 16.29 & 17.35 & 1.06 & 0.07 & 0.32 & 3.49 & 3.59 & -22.5 & 30.3 & 0.81 & 0.150 & I \\
\hline 155930.509 & +265535.00 & $\mathrm{gE}$ & 16.70 & 18.37 & 1.67 & 0.12 & 0.45 & 1.19 & 1.43 & -19.6 & 29.1 & 0.83 & 0.064 & I \\
\hline 160508.872 & +323922.43 & $\mathrm{gE}$ & 16.00 & 17.83 & 1.83 & 0.10 & 1.08 & 1.07 & 1.03 & -20.9 & 29.3 & 0.49 & 0.091 & \\
\hline 160545.860 & +412040.77 & $\mathrm{gE}$ & 9.24 & 9.30 & 0.06 & 0.03 & 0.71 & 2.38 & 4.36 & -24.6 & 28.0 & -2.30 & 0.006 & $\mathrm{I}, \mathrm{B}=13.9$ \\
\hline 160801.121 & +281150.82 & $*$ & 16.83 & 18.16 & 1.33 & 0.10 & 1.07 & 1.26 & 1.22 & -21.3 & 29.7 & 0.68 & 0.125 & \\
\hline 160957.973 & +314605.07 & $\mathrm{gO}$ & 16.49 & 18.25 & 1.76 & 0.09 & 0.36 & 1.02 & 1.28 & -19.5 & 29.0 & 0.74 & 0.059 & I \\
\hline 161224.728 & +383921.83 & $\mathrm{gE}$ & 16.34 & 17.14 & 0.80 & 0.04 & 0.51 & 1.35 & 0.83 & -22.0 & 29.6 & 0.31 & 0.110 & I \\
\hline 161259.828 & +421940.02 & $\mathrm{gE}$ & 17.06 & 18.16 & 1.09 & 0.04 & 0.53 & 3.44 & 3.60 & -22.7 & 30.6 & 1.12 & 0.230 & I \\
\hline 161407.633 & +352057.74 & $\mathrm{gO}$ & 17.78 & 19.15 & 1.37 & 0.05 & 0.05 & 4.37 & 4.07 & -20.3 & 30.2 & 1.62 & 0.125 & I \\
\hline 161423.557 & +401628.55 & $\mathrm{gO}$ & 17.33 & 18.38 & 1.04 & 0.04 & 0.23 & 1.07 & 1.27 & -20.8 & 29.5 & 0.77 & 0.110 & I \\
\hline 161756.888 & +225644.23 & $\mathrm{gO}$ & 7.89 & 9.37 & 1.48 & 0.27 & 0.71 & 10.72 & 11.39 & -25.4 & 28.8 & -1.85 & 0.015 & I,B \\
\hline 161941.046 & +274940.52 & $\mathrm{gO}$ & 17.10 & 18.47 & 1.37 & 0.17 & 0.69 & 1.73 & 1.69 & -20.9 & 29.8 & 0.95 & 0.120 & I \\
\hline 161941.951 & +282730.40 & $\mathrm{gO}$ & 16.03 & 16.82 & 0.80 & 0.15 & 0.62 & 1.29 & 0.74 & -22.6 & 29.7 & 0.16 & 0.126 & \\
\hline 162104.775 & +365446.26 & $\mathrm{gE}$ & 17.47 & 19.42 & 1.95 & 0.04 & 0.36 & 1.15 & 0.74 & -21.6 & 30.2 & 1.12 & 0.249 & \\
\hline 162125.089 & +293149.83 & $\mathrm{gO}$ & 16.82 & 18.33 & 1.51 & 0.12 & 0.63 & 1.30 & 0.84 & -22.1 & 30.0 & 0.75 & 0.192 & \\
\hline 162129.761 & +340746.95 & $\mathrm{gE}$ & 16.18 & 17.94 & 1.77 & 0.05 & 0.15 & 11.98 & 12.55 & -21.9 & 30.8 & 1.59 & 0.147 & \\
\hline 162323.988 & +354349.09 & $\mathrm{gE}$ & 16.90 & 18.00 & 1.10 & 0.04 & 0.55 & 1.15 & 1.09 & -22.0 & 29.8 & 0.57 & 0.164 & \\
\hline 162346.771 & +31002 & $*$ & 17.03 & 18.54 & 1.51 & 0.06 & 1.06 & 8 & 1.08 & -20.9 & 29.6 & 77 & 25 & I \\
\hline 162514.978 & +340624.76 & $\mathrm{gE}$ & 17.32 & 18.97 & 1.66 & 0.05 & 0.77 & 1.49 & 0.91 & -21.3 & 30.0 & 1.07 & 0.180 & I \\
\hline 162831.673 & +353120.27 & $\mathrm{gE}$ & 16.55 & 17.96 & 1.41 & 0.05 & 0.86 & 1.23 & 3.46 & -20.9 & 29.9 & 1.05 & 0.097 & I \\
\hline 163216.035 & +245832.20 & $*$ & 17.60 & 19.33 & 1.73 & 0.11 & 0.29 & 1.20 & 1.49 & -20.4 & 29.8 & 1.22 & 0.140 & \\
\hline 163546.935 & +285004.72 & $\mathrm{gO}$ & 17.37 & 18.24 & 0.87 & 0.08 & 0.48 & 3.62 & 4.39 & -21.6 & 30.3 & 1.25 & 0.147 & \\
\hline 163756.106 & +275402.64 & $\mathrm{gO}$ & 15.05 & 15.47 & 0.42 & 0.16 & 0.48 & 2.15 & 1.72 & -22.3 & 29.2 & -0.15 & 0.058 & I \\
\hline 163931.758 & +292744.60 & $\mathrm{gO}$ & 16.51 & 17.78 & 1.27 & 0.09 & 0.45 & 1.14 & 0.96 & -22.4 & 29.9 & 0.48 & 0.174 & \\
\hline 164705.980 & +293918.14 & $\mathrm{gO}$ & 14.53 & 15.40 & 0.87 & 0.12 & 0.23 & 2.13 & 2.66 & -22.1 & 29.2 & -0.08 & 0.053 & I \\
\hline 164934.541 & +375104.29 & $*$ & 16.75 & 18.12 & 1.37 & 0.07 & 0.35 & 1.35 & 1.41 & -21.9 & 29.9 & 0.71 & 0.164 & I \\
\hline 165423.486 & +331803.12 & $*$ & 17.23 & 17.74 & 0.51 & 0.07 & 0.26 & 3.59 & 3.64 & -21.9 & 30.2 & 0.97 & 0.140 & I \\
\hline 165435.303 & +263842.63 & $*$ & 17.65 & 18.51 & 0.86 & 0.21 & 0.41 & 1.22 & 0.87 & -20.9 & 29.6 & 0.81 & 0.121 & \\
\hline 165718.761 & +242844.15 & $*$ & 17.73 & 18.52 & 0.79 & 0.14 & 0.41 & 2.69 & 3.20 & -22.1 & 30.5 & 1.21 & 0.210 & I \\
\hline 170630.780 & +230130.69 & $\mathrm{gO}$ & 17.54 & 18.87 & 1.33 & 0.15 & 0.36 & 1.01 & 1.57 & -20.8 & 29.8 & 1.06 & 0.137 & \\
\hline 171240.358 & +331647.00 & $\mathrm{gE}$ & 12.80 & 13.96 & 1.16 & 0.11 & 0.42 & 4.32 & 8.23 & -24.0 & 29.9 & -0.17 & 0.063 & I \\
\hline 171310.239 & +262715.13 & $\mathrm{gE}$ & 12.97 & 13.98 & 1.01 & 0.11 & 0.54 & 1.31 & 1.97 & -23.3 & 29.0 & -0.78 & 0.048 & I \\
\hline 171756.257 & +380009.10 & $\mathrm{gE}$ & 17.53 & 19.10 & 1.57 & 0.13 & 0.16 & 1.19 & 1.51 & -21.3 & 30.1 & 1.12 & 0.190 & \\
\hline 171939.431 & +391537.99 & $\mathrm{gO}$ & 17.24 & 17.52 & 0.28 & 0.11 & 1.11 & 1.04 & 1.75 & -21.2 & 29.5 & 0.58 & 0.090 & \\
\hline 172241.861 & +362724.95 & $\mathrm{gO}$ & 17.44 & 18.01 & 0.58 & 0.13 & 0.64 & 1.62 & 1.30 & -22.4 & 30.2 & 0.72 & 0.197 & \\
\hline 172411.211 & +335206.05 & $\mathrm{gO}$ & 17.38 & 18.95 & 1.57 & 0.12 & 0.57 & 1.23 & 0.65 & -20.6 & 29.7 & 0.99 & 0.130 & \\
\hline 172438.106 & +315942.75 & $*$ & 16.65 & 17.93 & 1.28 & 0.12 & 1.03 & 1.01 & 1.89 & -21.0 & 29.6 & 0.77 & 0.101 & $\mathrm{E}$ (APS) \\
\hline 173604.538 & +401717.95 & $\mathrm{gE}$ & 17.72 & 19.05 & 1.33 & 0.10 & 0.76 & 1.37 & 1.94 & -21.9 & 30.4 & 1.20 & 0.240 & \\
\hline
\end{tabular}

Note.-Table columns are same as Table 2. 
TABLE 6

Passive Galaxies in FBQS CANDIDATE List

\begin{tabular}{|c|c|c|c|c|c|c|c|c|c|c|c|c|c|c|}
\hline $\begin{array}{l}\text { RA } \\
(1)\end{array}$ & $\begin{array}{c}\text { Dec } \\
(2)\end{array}$ & $\begin{array}{l}\mathrm{Cl} \\
(3)\end{array}$ & $\begin{array}{c}E \\
(4)\end{array}$ & $\begin{array}{c}\mathrm{O} \\
(5)\end{array}$ & $\begin{array}{c}\text { O-E } \\
(6)\end{array}$ & $\begin{array}{c}\mathrm{A}(\mathrm{E}) \\
(7)\end{array}$ & $\begin{array}{c}\text { Sep } \\
(8)\end{array}$ & $\begin{array}{l}S_{p} \\
(9)\end{array}$ & $\begin{array}{c}S_{i} \\
(10)\end{array}$ & $\begin{array}{c}M_{B} \\
(11)\end{array}$ & $\begin{array}{c}\log L_{R} \\
\text { (12) }\end{array}$ & $\begin{array}{c}\log R^{*} \\
(13)\end{array}$ & $\begin{array}{c}\text { Z } \\
(14)\end{array}$ & $\begin{array}{l}\text { Notes } \\
(15)\end{array}$ \\
\hline 071512.160 & +341658.70 & $\mathrm{gO}$ & 17.33 & 9.21 & 1.88 & 0.13 & 0.66 & 1.16 & 1.17 & -21.2 & 30.0 & 1.06 & 0.191 & \\
\hline 072853.441 & +334908.75 & $\mathrm{gO}$ & 7.56 & 7.74 & 0.18 & 0.16 & 0.54 & 37.95 & 64.61 & -26.9 & 29.5 & -1.75 & 0.014 & $\mathrm{o}, \mathrm{I}, \mathrm{B}=14.7$ \\
\hline 074819.128 & +345326.27 & $\mathrm{gO}$ & 17.68 & 19.40 & 1.72 & 0.16 & 0.32 & 1.18 & 1.48 & -20.1 & 29.8 & 1.25 & 0.130 & \\
\hline 075938.825 & +245856.57 & $\mathrm{gO}$ & 7.62 & 8.04 & 0.42 & 0.11 & 0.13 & 8.32 & 14.61 & -26.9 & 28.9 & -2.28 & 0.016 & $\mathrm{o}, \mathrm{I}, \mathrm{B}=14.3$ \\
\hline 083211.304 & +223338.03 & $\mathrm{gO}$ & 9.33 & 9.52 & 0.20 & 0.09 & 0.62 & 2.96 & 3.11 & -25.4 & 28.3 & -2.36 & 0.016 & $\mathrm{I}, \mathrm{B}=13.1$ \\
\hline 084859.624 & +410011.37 & $\mathrm{gO}$ & 7.93 & 9.49 & 1.56 & 0.08 & 0.91 & 1.84 & 2.49 & -26.3 & 28.5 & -2.47 & 0.024 & $B=14.9$ \\
\hline 085011.819 & +350435.56 & $\mathrm{gO}$ & 8.81 & 10.11 & 1.31 & 0.10 & 1.10 & 2.71 & 3.39 & -23.8 & 27.9 & -2.08 & 0.008 & $\mathrm{o}, \mathrm{I}, \mathrm{B}=13.9$ \\
\hline 091651.333 & +294124.45 & $*$ & 17.75 & 19.39 & 1.64 & 0.06 & 0.29 & 1.89 & 1.20 & $\cdots$ & & 1.38 & & \\
\hline 092207.845 & +283707.89 & $\mathrm{gO}$ & 17.45 & 19.16 & 1.71 & 0.06 & 0.23 & 1.71 & 2.38 & -22.2 & 30.7 & 1.33 & 0.294 & \\
\hline 092734.763 & +324713.55 & $\mathrm{gE}$ & 17.71 & 18.72 & 1.01 & 0.05 & 0.41 & 1.54 & 2.20 & -22.0 & 30.4 & 1.13 & 0.221 & $\mathrm{o}, \mathrm{I}$ \\
\hline 101258.369 & +393238.98 & $\mathrm{gE}$ & 16.30 & 17.77 & 1.47 & 0.04 & 0.35 & 20.97 & 20.65 & -22.4 & 31.1 & 1.74 & 0.171 & $X$ \\
\hline 102100.497 & +425907.54 & $\mathrm{gO}$ & 8.69 & 9.17 & 0.48 & 0.05 & 0.31 & 1.65 & 2.13 & -26.5 & 28.4 & -2.66 & 0.023 & $\mathrm{o}, \mathrm{B}=$ \\
\hline 104524.310 & +291308.69 & $\mathrm{gO}$ & 10.20 & 11.56 & 1.36 & 0.09 & 0.92 & 1.20 & 5.00 & -24.0 & 28.7 & -1.34 & 0.021 & $\mathrm{o}, \mathrm{I}, \mathrm{B}=14.9$ \\
\hline 110021.010 & +421053.09 & $*$ & 17.40 & 19.06 & 1.66 & 0.03 & 0.27 & 11.95 & 12.95 & -22.5 & 31.5 & 2.02 & 0.323 & \\
\hline 111524.467 & +415346.01 & $\mathrm{gE}$ & 17.64 & 18.94 & 1.30 & 0.04 & 0.12 & 1.55 & 1.31 & -22.5 & 30.5 & 1.05 & 0.306 & \\
\hline 111806.635 & +232350.76 & $\mathrm{gO}$ & 9.81 & 10.35 & 0.54 & 0.05 & 0.85 & 2.08 & 2.07 & -30.4 & 30.4 & -2.24 & 0.220 & $\mathrm{o}, \mathrm{B}=13.8$ \\
\hline 112617.267 & +352025.08 & $\mathrm{gO}$ & 11.01 & 11.69 & 0.68 & 0.06 & 0.48 & 4.83 & 4.92 & -24.9 & 29.1 & -1.29 & 0.034 & $B=15.3$ \\
\hline 112634.896 & +342921.47 & $\mathrm{gE}$ & 15.83 & 17.21 & 1.38 & 0.07 & 0.85 & 1.04 & 0.90 & -22.4 & 29.7 & 0.22 & 0.138 & \\
\hline 113731.056 & +312144.94 & $\mathrm{gE}$ & 9.33 & 8.63 & -0.70 & 0.06 & 1.01 & 1.06 & 13.57 & -28.5 & 29.8 & -2.08 & 0.043 & $B=14.6$ \\
\hline 114236.867 & +380014.52 & $\mathrm{gO}$ & 16.21 & 17.99 & 1.77 & 0.06 & 0.69 & & 0.83 & -21.5 & 29.6 & 0.53 & 30 & \\
\hline 114701.842 & +320351.49 & $\mathrm{gO}$ & 15.53 & 17.41 & 1.87 & 0.05 & 0.28 & 2.10 & 1.98 & -22.2 & 29.9 & 0.60 & 0.133 & \\
\hline 114957.179 & +374039.09 & $\mathrm{gO}$ & 15.22 & 16.22 & 1.00 & 0.06 & 1.17 & 1.28 & 1.48 & -23.6 & 29.9 & -0.03 & 0.148 & \\
\hline 115736.856 & +321638.98 & $\mathrm{gO}$ & 9.73 & 10.17 & 0.45 & 0.06 & 0.85 & 12.50 & 49.45 & -23.7 & 29.1 & -0.90 & 0.010 & 3.3 \\
\hline 115759.294 & +231206.89 & $\mathrm{gO}$ & 6.90 & 8.47 & 1.56 & 0.07 & 0.93 & 2.93 & 3.05 & -27.2 & 28.6 & -2.79 & 0.023 & $B=15.0$ \\
\hline 121049.612 & +392822.24 & $\mathrm{gO}$ & 8.52 & 9.24 & 0.72 & 0.07 & 1.12 & 2.46 & 2.93 & -26.4 & 28.5 & -2.50 & 0.022 & $\mathrm{o}, \mathrm{B}=14.0$ \\
\hline 122139.193 & +405056.15 & $\mathrm{gO}$ & 8.55 & 9.32 & 0.77 & 0.06 & 0.57 & 1.20 & 0.76 & -26.4 & 28.2 & -2.85 & 0.023 & $\mathrm{o}, \mathrm{I}, \mathrm{B}=13.8$ \\
\hline 125151.516 & +380539.41 & $\mathrm{gO}$ & 16.88 & 18.59 & 1.71 & 0.05 & 0.21 & 1.13 & 1.14 & -21.3 & 29.8 & 0.81 & 0.150 & \\
\hline 125735.823 & +272936.50 & $\mathrm{gO}$ & 10.35 & 11.69 & 1.34 & 0.03 & 0.82 & 1.13 & 0.96 & -24.1 & 28.2 & -1.93 & 0.024 & $\mathrm{~B}=$ \\
\hline 130617.310 & +290346.33 & $*$ & 8.58 & 9.10 & 0.52 & 0.03 & 1.01 & 1.45 & 14.97 & -26.7 & 29.3 & -1.84 & 0.024 & $\mathrm{I}, \mathrm{B}=13.9$ \\
\hline 131612.398 & +305701.05 & $\mathrm{gE}$ & 8.65 & 8.72 & 0.08 & 0.03 & 1.15 & 1.75 & 4.26 & -26.6 & 28.5 & -2.54 & 0.019 & $\mathrm{o}, \mathrm{I}, \mathrm{B}=13.7$ \\
\hline 132526.444 & +323607.52 & $*$ & 15.86 & 16.73 & 0.87 & 0.04 & 0.24 & 1.13 & 0.86 & $\ldots$ & $\cdots$ & 0.09 & & $\mathrm{O}$ (APS),o \\
\hline 133021.396 & +272607.44 & $\mathrm{gO}$ & 17.52 & 19.14 & 1.62 & 0.03 & 0.33 & 1.08 & 1.39 & -20.4 & 29.7 & 1.12 & 0.130 & I \\
\hline 133420.198 & +225138.68 & $\mathrm{gO}$ & 16.28 & 17.50 & 1.22 & 0.05 & 0.25 & 1.18 & 2.03 & -21.7 & 29.8 & 0.63 & 0.111 & \\
\hline 134438.001 & +33 3630.26 & $*$ & 17.14 & 18.83 & 1.69 & 0.06 & 1.00 & 1.06 & 1.06 & -22.1 & 30.1 & 0.86 & 0.240 & \\
\hline 135326.693 & +401658.91 & $\mathrm{gE}$ & 10.22 & 8.45 & -1.77 & 0.03 & 0.94 & 36.19 & 38.30 & -25.4 & 28.9 & -1.69 & 0.008 & $\mathrm{o}, \mathrm{B}=$ \\
\hline 135338.411 & +360802.61 & $\mathrm{gO}$ & 8.90 & 9.80 & 0.91 & 0.04 & 0.06 & 8.53 & 26.40 & -26.3 & 29.6 & -1.32 & 0.027 & $\mathrm{o}, \mathrm{B}=$ \\
\hline 135546.357 & +250907.87 & $\mathrm{gO}$ & 8.92 & 10.01 & 1.09 & 0.05 & 0.64 & 1.35 & 1.35 & -26.4 & 28.5 & -2.53 & 0.032 & \\
\hline 135604.438 & +381815.27 & $*$ & 10.36 & 9.68 & -0.68 & 0.05 & 0.14 & 2.76 & 2.65 & -25.6 & 28.4 & -2.35 & 0.019 & $\mathrm{o}, \mathrm{I}, \mathrm{B}=15.0$ \\
\hline 140045.814 & +300433.53 & $\mathrm{gO}$ & 8.56 & 9.35 & 0.79 & 0.04 & 0.91 & 1.92 & 2.12 & -26.7 & 28.5 & -2.60 & 0.027 & $\mathrm{o}, \mathrm{I}, \mathrm{B}=15.1$ \\
\hline 141218.458 & +253149.76 & $\mathrm{gO}$ & 17.51 & 19.21 & 1.70 & 0.06 & 0.29 & .02 & 1.42 & -20.9 & 30.0 & 1.14 & 0.170 & \\
\hline 143615.007 & +250532.52 & $\mathrm{gO}$ & 15.56 & 16.53 & 0.97 & 0.08 & 0.44 & 1.08 & 1.41 & -22.2 & 29.4 & 0.09 & 0.090 & \\
\hline 152408.011 & +324406.01 & $\mathrm{gO}$ & 14.82 & 16.54 & 1.72 & 0.06 & 0.73 & 1.97 & 2.52 & -22.7 & 29.9 & 0.34 & 0.112 & I \\
\hline 152944.096 & +424640.06 & $\mathrm{gO}$ & 9.63 & 10.00 & 0.36 & 0.08 & 0.92 & 1.90 & 2.13 & & & -2.33 & & $\mathrm{o}, \mathrm{I}$ \\
\hline 153552.136 & +394608.25 & $\mathrm{gO}$ & 9.07 & 10.93 & 1.86 & 0.06 & 0.58 & 2.94 & 2.99 & -23.8 & 28.2 & -1.81 & 0.015 & $\mathrm{o}, \mathrm{B}=13.1$ \\
\hline 154427.619 & +410714.31 & $*$ & 9.00 & 10.18 & 1.17 & 0.06 & 1.14 & 3.49 & 14.07 & -26.3 & 29.5 & -1.44 & 0.032 & $\mathrm{o}, \mathrm{I}, \mathrm{B}=13.9$ \\
\hline 160223.561 & +360635.39 & $\mathrm{gO}$ & 7.65 & 9.04 & 1.38 & 0.07 & 0.24 & 4.62 & 6.41 & -27.3 & 29.1 & -2.24 & 0.031 & $\mathrm{o}, \mathrm{B}=15.3$ \\
\hline 162550.029 & +402918.86 & $\mathrm{gO}$ & 10.86 & 8.70 & -2.16 & 0.02 & 1.04 & 25.82 & 29.55 & -27.5 & 29.8 & -1.71 & 0.029 & $B=15.0$ \\
\hline 163325.767 & +264052.02 & $\mathrm{gO}$ & 16.61 & 18.55 & 1.93 & 0.19 & 0.15 & 5.74 & 6.11 & -21.1 & 30.4 & 1.52 & 0.135 & \\
\hline 163750.344 & +292331.60 & $\mathrm{gO}$ & 17.45 & 19.42 & 1.97 & 0.06 & 0.21 & 1.25 & 0.91 & -19.4 & 29.4 & 1.19 & 0.093 & \\
\hline 165016.232 & +400924.63 & $\mathrm{gE}$ & 16.68 & 18.13 & 1.45 & 0.04 & 0.70 & 2.29 & 3.78 & -22.1 & 30.4 & 1.14 & 0.178 & \\
\hline 165643.187 & +274919.42 & $\mathrm{gO}$ & 9.39 & 8.92 & -0.47 & 0.15 & 0.87 & 1.54 & 2.69 & -27.7 & 28.9 & -2.67 & 0.035 & $B=14.7$ \\
\hline 171455.118 & +3531 12.39 & $\mathrm{gE}$ & 8.26 & 8.84 & 0.58 & 0.08 & 1.19 & 1.47 & 5.58 & -27.9 & 29.2 & -2.38 & 0.037 & $\mathrm{o}, \mathrm{I}, \mathrm{B}=14.9$ \\
\hline
\end{tabular}

Note.-Table columns are same as Table 2. 
TABLE 7

STARS IN FBQS CANDIDATE LIST

\begin{tabular}{|c|c|c|c|c|c|c|c|c|c|c|}
\hline $\begin{array}{l}\text { RA } \\
\text { (1) }\end{array}$ & $\begin{array}{l}\text { Dec } \\
\text { (2) }\end{array}$ & $\begin{array}{l}\mathrm{Cl} \\
\text { (3) }\end{array}$ & $\begin{array}{c}\text { E } \\
(4)\end{array}$ & $\begin{array}{c}\mathrm{O} \\
(5)\end{array}$ & $\begin{array}{l}\mathrm{O}-\mathrm{E} \\
(6)\end{array}$ & $\begin{array}{c}\mathrm{A}(\mathrm{E}) \\
(7)\end{array}$ & $\begin{array}{c}\text { Sep } \\
(8)\end{array}$ & $\begin{array}{l}S_{p} \\
(9)\end{array}$ & $\begin{array}{c}S_{i} \\
(10)\end{array}$ & $\begin{array}{l}\text { Notes } \\
\text { (11) }\end{array}$ \\
\hline 065106.134 & +311902.00 & * & 16.57 & 18.15 & 1.58 & 0.29 & 1.06 & 7.29 & 9.64 & $\mathrm{E}(\mathrm{APS}), \mathrm{O}(\mathrm{APS})$ \\
\hline 072307.316 & +282646.57 & * & 16.77 & 18.03 & 1.26 & 0.22 & 0.90 & 1.39 & 0.95 & \\
\hline 072408.835 & +350033.01 & * & 17.74 & 19.32 & 1.58 & 0.14 & 0.91 & 8.77 & 9.49 & \\
\hline 072709.552 & +383139.32 & * & 14.54 & 14.87 & 0.33 & 0.17 & 1.14 & 1.42 & 1.66 & \\
\hline 072735.830 & +373452.58 & $\mathrm{gE}$ & 17.60 & 18.59 & 0.99 & 0.15 & 0.14 & 6.10 & 6.81 & ? \\
\hline 074159.817 & +233158.12 & * & 15.59 & 16.49 & 0.90 & 0.15 & 0.82 & 1.25 & 1.55 & \\
\hline 074605.795 & +330847.46 & * & 15.66 & 16.41 & 0.75 & 0.14 & 0.80 & 24.36 & 42.52 & \\
\hline 074629.102 & +392652.78 & * & 16.29 & 17.35 & 1.06 & 0.15 & 0.84 & 1.68 & 1.73 & \\
\hline 074809.719 & +370926.38 & * & 15.52 & 17.05 & 1.53 & 0.24 & 0.52 & 1.05 & 1.26 & \\
\hline 074916.459 & +391722.13 & $\mathrm{gE}$ & 14.11 & 16.00 & 1.89 & 0.13 & 0.92 & 1.10 & 3.53 & \\
\hline 074956.001 & +35 5631.14 & * & 15.97 & 16.15 & 0.18 & 0.16 & 0.60 & 1.29 & 2.38 & \\
\hline 075413.730 & +393720.19 & * & 15.32 & 16.07 & 0.75 & 0.14 & 0.33 & 1.27 & 1.29 & \\
\hline 075436.440 & +310252.37 & * & 15.71 & 17.19 & 1.49 & 0.14 & 1.17 & 3.14 & 5.70 & \\
\hline 075715.873 & +301012.86 & * & 16.57 & 18.36 & 1.79 & 0.13 & 0.21 & 3.57 & 3.82 & \\
\hline 075804.473 & +325719.92 & $\mathrm{gE}$ & 16.79 & 18.76 & 1.96 & 0.16 & 1.11 & 1.41 & 1.08 & \\
\hline 080106.610 & +315852.81 & * & 16.99 & 17.93 & 0.94 & 0.13 & 0.88 & 1.40 & 1.80 & \\
\hline 080925.573 & +351906.00 & * & 15.34 & 16.39 & 1.04 & 0.13 & 1.17 & 28.63 & 31.83 & \\
\hline 081008.685 & +391728.71 & * & 15.71 & 17.09 & 1.39 & 0.13 & 0.71 & 4.99 & 4.27 & \\
\hline 082008.253 & +345954.74 & * & 14.25 & 14.68 & 0.43 & 0.15 & 0.38 & 4.42 & 4.81 & \\
\hline 084807.053 & +280416.29 & * & 14.51 & 15.12 & 0.61 & 0.13 & 1.04 & 1.56 & 2.07 & \\
\hline 085418.535 & +2811 19.05 & * & 15.59 & 16.17 & 0.58 & 0.10 & 1.06 & 1.39 & 1.04 & \\
\hline 085452.606 & +222300.55 & * & 16.77 & 17.66 & 0.90 & 0.09 & 0.21 & 3.15 & 3.00 & \\
\hline 090652.129 & +285550.24 & * & 16.52 & 17.36 & 0.84 & 0.08 & 0.92 & 2.49 & 5.32 & \\
\hline 091033.513 & +345123.24 & * & 16.68 & 17.68 & 1.01 & 0.06 & 0.83 & 3.19 & 2.86 & \\
\hline 093253.265 & +353744.21 & * & 8.73 & 10.49 & 1.75 & 0.04 & 1.19 & 3.00 & 4.72 & \\
\hline 093850.594 & +305134.83 & $\mathrm{gO}$ & 11.87 & 12.89 & 1.02 & 0.05 & 0.74 & 1.34 & 1.54 & \\
\hline 094237.334 & +240054.06 & * & 15.33 & 17.23 & 1.90 & 0.08 & 0.57 & 1.15 & 1.26 & \\
\hline 095100.691 & +361432.55 & * & 11.76 & 13.09 & 1.33 & 0.03 & 0.92 & 3.17 & 3.34 & \\
\hline 095926.024 & +275955.20 & * & 13.52 & 15.08 & 1.56 & 0.05 & 0.53 & 9.02 & 8.85 & \\
\hline 102849.259 & +331519.25 & $\mathrm{gE}$ & 16.83 & 18.67 & 1.84 & 0.05 & 0.68 & 1.22 & 1.27 & \\
\hline 103954.084 & +313358.56 & * & 14.80 & 15.93 & 1.13 & 0.06 & 0.61 & 1.34 & 9.17 & \\
\hline 111326.652 & +351551.51 & * & 17.72 & 19.55 & 1.82 & 0.05 & 1.05 & 1.70 & 1.07 & \\
\hline 111810.749 & +275425.12 & * & 15.78 & 16.70 & 0.92 & 0.05 & 0.72 & 1.23 & 13.19 & \\
\hline 111858.635 & +382851.96 & * & 17.68 & 17.78 & 0.10 & 0.05 & 0.52 & 4.22 & 4.84 & \\
\hline 111904.943 & +295152.91 & * & 14.55 & 14.12 & -0.43 & 0.04 & 1.05 & 4.40 & 4.99 & \\
\hline 112442.063 & +303637.65 & * & 11.80 & 12.99 & 1.19 & 0.04 & 0.71 & 12.04 & 43.23 & \\
\hline 114654.298 & +323652.24 & * & 17.33 & 18.11 & 0.78 & 0.05 & 0.36 & 14.67 & 15.42 & \\
\hline 115619.683 & +331840.95 & * & 16.80 & 18.74 & 1.94 & 0.05 & 0.42 & 28.78 & 39.27 & \\
\hline 115824.302 & +221913.74 & * & 16.86 & 17.50 & 0.64 & 0.07 & 0.79 & 1.85 & 1.54 & \\
\hline 120447.021 & +293210.67 & * & 17.25 & 18.95 & 1.70 & 0.06 & 0.88 & 1.25 & 0.65 & \\
\hline 121405.981 & +372610.78 & $\mathrm{gO}$ & 16.86 & 17.52 & 0.66 & 0.06 & 0.81 & 1.62 & 1.88 & \\
\hline 123129.767 & +234301.40 & * & 11.37 & 13.07 & 1.70 & 0.05 & 0.42 & 1.10 & 1.01 & \\
\hline 123645.931 & +283518.84 & * & 16.63 & 17.37 & 0.74 & 0.05 & 0.41 & 1.47 & 1.04 & \\
\hline 124044.501 & +231045.79 & * & 17.68 & 18.41 & 0.73 & 0.06 & 0.27 & 40.76 & 65.39 & \\
\hline 124109.824 & +240810.50 & * & 16.73 & 17.63 & 0.90 & 0.05 & 1.15 & 2.47 & 3.00 & \\
\hline 125722.637 & +393726.60 & * & 14.29 & 15.28 & 0.99 & 0.04 & 0.68 & 1.19 & 1.04 & \\
\hline 140414.703 & +284636.87 & * & 16.16 & 17.36 & 1.21 & 0.04 & 1.12 & 11.92 & 12.43 & \\
\hline 141615.598 & +354450.48 & $*$ & 17.25 & 19.06 & 1.81 & 0.03 & 1.16 & 1.25 & 1.55 & \\
\hline 141716.102 & +371444.75 & * & 16.24 & 17.91 & 1.66 & 0.03 & 0.33 & 25.92 & 26.34 & \\
\hline 142136.867 & +353118.71 & * & 15.16 & 16.22 & 1.05 & 0.04 & 0.56 & 1.08 & 3.04 & \\
\hline 142142.198 & +331935.64 & * & 16.86 & 18.04 & 1.18 & 0.04 & 0.51 & 1.12 & 1.33 & \\
\hline 142455.517 & +361536.25 & * & 17.41 & 17.95 & 0.54 & 0.02 & 0.25 & 85.17 & 87.77 & \\
\hline 143205.098 & +402131.50 & * & 16.90 & 18.57 & 1.67 & 0.04 & 0.66 & 1.25 & 1.96 & \\
\hline 144048.122 & +402823.50 & $\mathrm{gO}$ & 16.28 & 17.14 & 0.86 & 0.04 & 0.50 & 1.00 & 1.25 & \\
\hline 144425.312 & +334649.70 & * & 15.43 & 16.92 & 1.49 & 0.03 & 0.83 & 1.29 & 0.84 & \\
\hline 144932.821 & +301205.26 & * & 17.30 & 18.04 & 0.74 & 0.05 & 1.18 & 10.67 & 16.99 & \\
\hline 150434.577 & +252731.90 & * & 17.44 & 18.34 & 0.90 & 0.10 & 0.75 & 6.10 & 6.49 & \\
\hline 150717.016 & +231443.40 & * & 17.58 & 19.41 & 1.83 & 0.14 & 0.82 & 2.97 & 7.14 & \\
\hline 151136.630 & +362444.30 & * & 12.75 & 13.83 & 1.08 & 0.05 & 1.03 & 2.48 & 2.94 & \\
\hline 152512.532 & +351919.40 & * & 16.62 & 18.19 & 1.57 & 0.05 & 0.82 & 2.72 & 3.68 & \\
\hline 153023.047 & +42 4329.40 & $\mathrm{gE}$ & 17.00 & 18.72 & 1.72 & 0.08 & 0.38 & 1.57 & 2.22 & \\
\hline 155355.861 & +265917.21 & * & 16.50 & 17.71 & 1.21 & 0.11 & 1.04 & 2.06 & 2.17 & \\
\hline 161438.412 & +383058.15 & * & 16.95 & 18.00 & 1.05 & 0.04 & 0.97 & 1.38 & 1.77 & \\
\hline 161635.697 & +350928.53 & $\mathrm{gE}$ & 14.82 & 15.68 & 0.86 & 0.06 & 0.58 & 1.35 & 2.50 & I \\
\hline 161846.174 & +325127.29 & * & 16.55 & 17.76 & 1.20 & 0.06 & 0.73 & 4.93 & 4.38 & \\
\hline 161851.744 & +221130.50 & $\mathrm{gE}$ & 15.33 & 16.49 & 1.15 & 0.22 & 0.89 & 4.57 & 5.66 & \\
\hline 164018.144 & +384220.00 & * & 12.76 & 13.09 & 0.32 & 0.03 & 0.59 & 4.78 & 4.05 & $\mathrm{I}, \mathrm{PN}$ \\
\hline 170008.695 & +291904.11 & $\mathrm{gO}$ & 16.14 & 17.33 & 1.19 & 0.12 & 0.98 & 3.28 & 3.02 & \\
\hline 170127.814 & +232025.26 & * & 16.64 & 17.32 & 0.68 & 0.15 & 0.67 & 1.04 & 1.75 & \\
\hline 170411.600 & +293153.70 & * & 15.42 & 17.04 & 1.62 & 0.15 & 0.43 & 1.13 & 1.45 & \\
\hline 170718.543 & +380204.14 & * & 16.35 & 17.20 & 0.86 & 0.09 & 0.23 & 1.04 & 0.97 & \\
\hline
\end{tabular}


TABLE 7-Continued

\begin{tabular}{|c|c|c|c|c|c|c|c|c|c|c|}
\hline $\begin{array}{l}\text { RA } \\
\text { (1) }\end{array}$ & $\begin{array}{l}\text { Dec } \\
(2)\end{array}$ & $\begin{array}{l}\mathrm{Cl} \\
(3)\end{array}$ & $\begin{array}{l}E \\
(4)\end{array}$ & $\begin{array}{l}\mathrm{O} \\
(5)\end{array}$ & $\begin{array}{l}\text { O-E } \\
(6)\end{array}$ & $\begin{array}{c}\mathrm{A}(\mathrm{E}) \\
(7)\end{array}$ & $\begin{array}{c}\text { Sep } \\
(8)\end{array}$ & $\begin{array}{l}S_{p} \\
(9)\end{array}$ & $\begin{array}{c}S_{i} \\
(10)\end{array}$ & $\begin{array}{l}\text { Notes } \\
\text { (11) }\end{array}$ \\
\hline 170726.440 & +282730.03 & $*$ & 14.89 & 15.92 & 1.04 & 0.27 & 0.36 & 2.18 & 4.42 & \\
\hline 170820.213 & +355300.64 & $*$ & 14.33 & 15.43 & 1.11 & 0.06 & 0.71 & 5.38 & 6.41 & \\
\hline 170912.986 & +353017.45 & $\mathrm{gO}$ & 14.20 & 14.03 & -0.17 & 0.06 & 0.99 & 1.38 & 2.17 & \\
\hline 170948.432 & +261314.78 & $*$ & 15.89 & 17.33 & 1.44 & 0.09 & 0.86 & 14.82 & 16.14 & \\
\hline 171038.017 & +373851.25 & $*$ & 15.92 & 17.76 & 1.85 & 0.11 & 0.93 & 18.45 & 23.17 & \\
\hline 171239.149 & +27 2028.77 & * & 17.02 & 18.47 & 1.45 & 0.12 & 1.02 & 2.22 & 2.03 & \\
\hline 171304.499 & +321324.18 & $\mathrm{gE}$ & 16.52 & 17.18 & 0.67 & 0.14 & 0.41 & 1.44 & 3.51 & \\
\hline 171309.366 & +221502.73 & $*$ & 14.80 & 16.00 & 1.20 & 0.14 & 0.58 & 1.38 & 1.34 & \\
\hline 171548.825 & +345746.21 & $\mathrm{gO}$ & 16.75 & 17.79 & 1.03 & 0.09 & 1.14 & 1.15 & 0.87 & I \\
\hline 171552.779 & +271815.24 & $\mathrm{gO}$ & 13.66 & 15.48 & 1.82 & 0.16 & 1.08 & 3.80 & 4.67 & \\
\hline 171641.366 & +331443.60 & $*$ & 15.36 & 16.57 & 1.21 & 0.12 & 1.16 & 2.84 & 2.27 & \\
\hline 171716.039 & +353105.75 & * & 17.54 & 18.71 & 1.17 & 0.10 & 0.41 & 1.20 & 3.30 & O(APS) \\
\hline 171748.208 & +393514.22 & $*$ & 12.48 & 14.47 & 1.99 & 0.11 & 0.80 & 2.67 & 5.21 & \\
\hline 171801.678 & +340449.35 & $*$ & 16.26 & 17.20 & 0.94 & 0.08 & 0.95 & 1.94 & 1.61 & \\
\hline 171948.610 & +351801.13 & $\mathrm{gE}$ & 15.20 & 17.11 & 1.91 & 0.09 & 1.00 & 9.09 & 10.19 & \\
\hline 172011.727 & +412826.57 & $\mathrm{gO}$ & 17.53 & 17.96 & 0.43 & 0.07 & 0.90 & 22.80 & 49.20 & \\
\hline 172247.472 & +320037.83 & $*$ & 16.75 & 17.70 & 0.95 & 0.11 & 0.90 & 1.91 & 1.45 & \\
\hline
\end{tabular}

NOTE.-Table columns are same as Table 2, except that redshift, absolute magnitudes, etc., are omitted for these Galactic objects. One object, marked PN in the notes column, is a halopopulation planetary nebula. 
TABLE 8

ObJects Without Spectra in FBQS Candidate List

\begin{tabular}{|c|c|c|c|c|c|c|c|c|c|c|c|}
\hline $\begin{array}{l}\text { RA } \\
\text { (1) }\end{array}$ & $\begin{array}{l}\text { Dec } \\
(2)\end{array}$ & $\begin{array}{l}\mathrm{Cl} \\
(3)\end{array}$ & $\begin{array}{c}E \\
(4)\end{array}$ & $\begin{array}{l}\mathrm{O} \\
(5)\end{array}$ & $\begin{array}{l}\text { O-E } \\
(6)\end{array}$ & $\begin{array}{c}\mathrm{A}(\mathrm{E}) \\
(7)\end{array}$ & $\begin{array}{l}\text { Sep } \\
(8)\end{array}$ & $\begin{array}{l}S_{p} \\
(9)\end{array}$ & $\begin{array}{c}S_{i} \\
(10)\end{array}$ & $\begin{array}{l}\mathrm{P}(\mathrm{Q}) \\
(11)\end{array}$ & $\begin{array}{l}\text { Notes } \\
\text { (12) }\end{array}$ \\
\hline 071505.450 & +340501.50 & $*$ & 17.43 & 18.28 & 0.85 & 0.13 & 1.00 & 2.15 & 2.61 & 0.43 & \\
\hline 071650.696 & +350520.30 & $\mathrm{gO}$ & 17.39 & 19.23 & 1.84 & 0.15 & 0.54 & 1.33 & 0.80 & 0.11 & \\
\hline 071903.275 & +342550.27 & $\mathrm{gE}$ & 15.19 & 16.23 & 1.04 & 0.14 & 1.15 & 9.81 & 21.65 & 0.12 & \\
\hline 073018.190 & +224502.12 & $\mathrm{gO}$ & 17.39 & 19.07 & 1.68 & 0.14 & 0.38 & 1.12 & 1.42 & 0.11 & \\
\hline 073237.941 & +342952.90 & $\mathrm{gE}$ & 17.76 & 19.68 & 1.91 & 0.14 & 0.24 & 1.30 & 1.98 & 0.11 & I \\
\hline 073317.396 & +223725.01 & $\mathrm{gO}$ & 17.33 & 19.13 & 1.81 & 0.19 & 0.28 & 1.68 & 3.20 & 0.11 & \\
\hline 073833.547 & +360957.12 & $\mathrm{gO}$ & 17.51 & 19.44 & 1.92 & 0.15 & 0.08 & 2.20 & 1.66 & 0.11 & \\
\hline 074342.266 & +321543.52 & $\mathrm{gO}$ & 17.34 & 18.46 & 1.11 & 0.12 & 1.05 & 2.78 & 2.44 & 0.15 & \\
\hline 082711.207 & +223323.50 & $\mathrm{gO}$ & 17.20 & 18.91 & 1.71 & 0.12 & 0.83 & 2.30 & 2.23 & 0.11 & \\
\hline 083522.735 & +424258.75 & * & 17.06 & 17.70 & 0.65 & 0.07 & 0.58 & 1.33 & 0.96 & 0.86 & \\
\hline 085552.740 & +384325.79 & $\mathrm{gO}$ & 17.53 & 19.11 & 1.58 & 0.07 & 0.44 & 1.51 & 0.93 & 0.11 & \\
\hline 085624.852 & +345024.83 & $\mathrm{gO}$ & 17.20 & 19.17 & 1.97 & 0.08 & 0.15 & 8.64 & 8.51 & 0.13 & I \\
\hline 091309.264 & +413635.18 & * & 16.33 & 17.56 & 1.23 & 0.06 & 1.14 & 1.03 & 2.59 & 0.31 & \\
\hline 091833.851 & +315620.45 & $\mathrm{gE}$ & 17.69 & 18.74 & 1.04 & 0.05 & 0.85 & 1.26 & 1.21 & 0.33 & $\mathrm{X}$ \\
\hline 091845.785 & +233833.53 & $\mathrm{gE}$ & 17.00 & 18.77 & 1.77 & 0.16 & 0.36 & 1.19 & 0.80 & 0.11 & \\
\hline 093456.726 & +263054.49 & $\mathrm{gO}$ & 17.70 & 19.20 & 1.49 & 0.05 & 0.95 & 1.02 & 1.21 & 0.11 & \\
\hline 101355.238 & +300546.81 & * & 17.76 & 18.46 & 0.69 & 0.08 & 0.90 & 1.38 & 2.22 & 0.65 & \\
\hline 102802.937 & +304743.55 & $*$ & 17.72 & 19.58 & 1.87 & 0.05 & 0.94 & 1.12 & 1.17 & 0.11 & \\
\hline 102857.616 & +344054.41 & $*$ & 17.76 & 19.66 & 1.90 & 0.05 & 1.03 & 1.41 & 1.45 & 0.11 & \\
\hline 103346.379 & +233220.09 & * & 17.51 & 18.40 & 0.88 & 0.06 & 0.20 & 8.97 & 8.93 & 0.87 & \\
\hline 103818.198 & +424442.85 & $\mathrm{gE}$ & 17.54 & 19.22 & 1.67 & 0.04 & 0.15 & 78.28 & 80.28 & 0.52 & \\
\hline 105330.963 & +331342.16 & $\mathrm{gE}$ & 17.19 & 19.10 & 1.91 & 0.06 & 0.30 & 2.72 & 2.69 & 0.11 & I \\
\hline 105426.029 & +423230.94 & $\mathrm{gE}$ & 17.53 & 18.79 & 1.26 & 0.04 & 0.32 & 3.36 & 4.05 & $\ldots$ & $\mathrm{E}(\mathrm{APS}), \mathrm{O}(\mathrm{APS})$ \\
\hline 105653.384 & +331945.12 & $\mathrm{gE}$ & 14.94 & 16.75 & 1.81 & 0.05 & 0.65 & 2.32 & 2.53 & 0.11 & I \\
\hline 110113.801 & +323155.22 & $\mathrm{gE}$ & 15.35 & 17.15 & 1.80 & 0.06 & 0.59 & 1.10 & 2.38 & 0.11 & \\
\hline 112242.834 & +414355.69 & $\mathrm{gO}$ & 16.99 & 18.66 & 1.67 & 0.05 & 1.13 & 1.34 & 2.37 & 0.11 & \\
\hline 112520.786 & +263749.55 & * & 17.68 & 18.49 & 0.81 & 0.05 & 0.73 & 30.98 & 36.53 & $\ldots$ & E(APS) \\
\hline 113020.404 & +422204.09 & $*$ & 17.77 & 18.53 & 0.76 & 0.05 & 0.34 & 2.01 & 2.27 & 0.87 & \\
\hline 113124.210 & +261951.45 & * & 17.64 & 18.12 & 0.48 & 0.05 & 0.50 & 3.65 & 3.48 & 0.88 & \\
\hline 113324.786 & +323449.30 & $*$ & 17.60 & 18.86 & 1.26 & 0.07 & 0.83 & 13.14 & 13.20 & 0.63 & \\
\hline 113442.086 & +411330.11 & $*$ & 17.80 & 18.07 & 0.28 & 0.06 & 0.15 & 42.45 & 47.04 & 0.88 & \\
\hline 113609.065 & +360641.72 & $\mathrm{gO}$ & 17.68 & 19.16 & 1.48 & 0.05 & 0.91 & 1.18 & 1.31 & 0.11 & \\
\hline 113639.177 & +372651.57 & $\mathrm{gE}$ & 17.01 & 18.20 & 1.19 & 0.07 & 0.39 & 17.53 & 21.80 & 0.29 & \\
\hline 113707.730 & +290324.27 & $*$ & 17.80 & 18.14 & 0.33 & 0.06 & 0.45 & 20.30 & 23.06 & 0.88 & \\
\hline 113921.210 & +350748.30 & * & 17.03 & 18.53 & 1.49 & 0.05 & 0.40 & 1.17 & 0.56 & 0.20 & \\
\hline 114048.084 & +332908.50 & $*$ & 17.71 & 18.31 & 0.60 & 0.06 & 0.19 & 21.07 & 22.73 & 0.88 & \\
\hline 114111.118 & +300442.66 & * & 17.18 & 17.72 & 0.54 & 0.06 & 0.75 & 1.00 & 1.35 & 0.84 & \\
\hline 115244.450 & +311123.88 & $\mathrm{gE}$ & 17.37 & 19.29 & 1.92 & 0.05 & 0.84 & 1.15 & 1.38 & 0.11 & \\
\hline 115943.881 & +303348.95 & $*$ & 17.54 & 18.92 & 1.38 & 0.04 & 0.51 & 1.49 & 2.01 & 0.11 & \\
\hline 120354.768 & +371137.36 & $*$ & 17.67 & 17.79 & 0.12 & 0.05 & 0.13 & 4.59 & 4.57 & 0.88 & $\mathrm{X}$ \\
\hline 120908.415 & +265131.94 & $\mathrm{gE}$ & 17.66 & 18.13 & 0.47 & 0.05 & 0.98 & 1.19 & 1.70 & 0.69 & \\
\hline 121147.120 & +240736.69 & * & 17.61 & 19.36 & 1.75 & 0.09 & 0.80 & 1.40 & 1.61 & 0.11 & \\
\hline $12 \quad 1232.373$ & +425821.99 & * & 17.40 & 18.43 & 1.03 & 0.04 & 0.05 & 37.58 & 41.96 & 0.87 & \\
\hline 121355.332 & +365255.74 & $*$ & 17.37 & 18.53 & 1.16 & 0.06 & 0.82 & 1.14 & 1.77 & 0.11 & \\
\hline 121529.648 & +391200.51 & $*$ & 17.65 & 19.26 & 1.61 & 0.05 & 1.12 & 1.01 & 1.32 & 0.11 & \\
\hline 121727.863 & +290449.59 & $\mathrm{gE}$ & 17.65 & 19.24 & 1.59 & 0.05 & 0.59 & 1.10 & 4.04 & 0.11 & \\
\hline 121902.540 & +222416.59 & $\mathrm{gE}$ & 17.78 & 19.65 & 1.86 & 0.07 & 0.03 & 7.32 & 7.49 & 0.31 & \\
\hline 122004.385 & +311148.08 & * & 17.52 & 17.95 & 0.43 & 0.05 & 0.05 & 26.21 & 28.41 & 0.88 & \\
\hline 122034.613 & +363357.38 & $\mathrm{gO}$ & 17.07 & 18.98 & 1.91 & 0.05 & 0.11 & 1.35 & 1.28 & 0.11 & \\
\hline 122208.184 & +240012.31 & $*$ & 17.66 & 19.26 & 1.60 & 0.06 & 0.26 & 7.21 & 7.12 & 0.14 & \\
\hline 122221.377 & +372335.73 & $\mathrm{gO}$ & 17.15 & 18.06 & 0.91 & 0.04 & 0.43 & 10.38 & 10.21 & 0.65 & \\
\hline 122251.382 & +331640.80 & $*$ & 17.45 & 19.08 & 1.63 & 0.04 & 0.59 & 2.55 & 2.96 & 0.56 & \\
\hline 122407.371 & +375332.65 & $*$ & 17.79 & 19.38 & 1.59 & 0.03 & 0.56 & 1.22 & 0.88 & 0.11 & \\
\hline 122520.497 & +292420.25 & $*$ & 17.76 & 19.04 & 1.28 & 0.06 & 0.46 & 2.30 & 1.93 & 0.85 & \\
\hline 122856.688 & +355635.38 & $*$ & 17.50 & 17.61 & 0.11 & 0.04 & 0.86 & 8.68 & 9.99 & 0.88 & \\
\hline 123659.550 & +423641.56 & * & 17.27 & 17.99 & 0.72 & 0.07 & 0.13 & 193.40 & 197.82 & 0.87 & I \\
\hline 123757.924 & +223430.41 & * & 17.65 & 18.03 & 0.37 & 0.06 & 0.32 & 155.96 & 176.43 & 0.88 & \\
\hline 124327.825 & +232811.35 & $\mathrm{gE}$ & 17.31 & 18.21 & 0.90 & 0.06 & 0.68 & 2.33 & 2.63 & 0.64 & I \\
\hline 124444.575 & +223305.35 & $\mathrm{gO}$ & 17.60 & 19.47 & 1.87 & 0.08 & 0.11 & 1.75 & 2.58 & 0.15 & \\
\hline 124840.446 & +241240.78 & $*$ & 17.50 & 18.12 & 0.61 & 0.04 & 0.13 & 2.91 & 3.40 & 0.88 & \\
\hline 124958.854 & +245233.40 & $*$ & 17.74 & 18.75 & 1.01 & 0.04 & 0.45 & 7.54 & 8.43 & 0.87 & \\
\hline 125018.184 & +364914.06 & $\mathrm{gO}$ & 17.68 & 19.58 & 1.91 & 0.05 & 1.00 & 1.49 & 1.15 & 0.11 & I \\
\hline 125142.267 & +240435.10 & * & 17.34 & 17.84 & 0.50 & 0.03 & 0.60 & 1.62 & 1.51 & 0.86 & $X$ \\
\hline 125256.997 & +252503.27 & * & 17.41 & 17.68 & 0.28 & 0.03 & 0.49 & 1.39 & 1.43 & 0.88 & \\
\hline 125444.719 & +425305.23 & $*$ & 17.15 & 18.16 & 1.01 & 0.05 & 0.06 & 1.95 & 1.62 & 0.84 & \\
\hline 131823.430 & +262623.00 & $\mathrm{gE}$ & 17.75 & 18.18 & 0.44 & 0.04 & 0.34 & 6.35 & 6.36 & 0.87 & \\
\hline 131848.337 & +252815.40 & $*$ & 17.65 & 18.26 & 0.62 & 0.04 & 0.90 & 1.34 & 1.44 & 0.70 & \\
\hline 132324.153 & +251809.58 & $\mathrm{gO}$ & 17.79 & 18.59 & 0.79 & 0.03 & 0.23 & 5.77 & 5.59 & 0.87 & \\
\hline 132557.665 & +33 1334.10 & $\mathrm{gO}$ & 17.40 & 19.30 & 1.90 & 0.03 & 0.54 & 1.41 & 2.01 & $\ldots$ & E(APS), \\
\hline 134034.727 & +423232.32 & * & 17.70 & 18.44 & 0.74 & 0.03 & 0.61 & 5.39 & 5.17 & .. & E(APS), O(APS) \\
\hline 134531.092 & +255504.47 & $*$ & 13.08 & 14.00 & 0.92 & 0.03 & 1.07 & 4.20 & 4.14 & 0.12 & \\
\hline
\end{tabular}


TABLE 8-Continued

\begin{tabular}{|c|c|c|c|c|c|c|c|c|c|c|c|}
\hline $\begin{array}{l}\text { RA } \\
\text { (1) }\end{array}$ & $\begin{array}{c}\text { Dec } \\
(2)\end{array}$ & $\begin{array}{l}\mathrm{Cl} \\
(3)\end{array}$ & $\begin{array}{c}E \\
(4)\end{array}$ & $\begin{array}{c}\mathrm{O} \\
(5)\end{array}$ & $\begin{array}{c}\text { O-E } \\
(6)\end{array}$ & $\begin{array}{c}\mathrm{A}(\mathrm{E}) \\
(7)\end{array}$ & $\begin{array}{c}\text { Sep } \\
(8)\end{array}$ & $\begin{array}{l}S_{p} \\
(9)\end{array}$ & $\begin{array}{c}S_{i} \\
(10)\end{array}$ & $\begin{array}{c}\mathrm{P}(\mathrm{Q}) \\
(11)\end{array}$ & $\begin{array}{l}\text { Notes } \\
\text { (12) }\end{array}$ \\
\hline 134540.043 & 23.35 & $\mathrm{gE}$ & 17.79 & 19.66 & 1.87 & 0.04 & 0.58 & 13.25 & 13.83 & 0.13 & \\
\hline 140819.354 & +294950.57 & $*$ & 17.78 & 18.12 & 0.34 & 0.04 & 0.06 & 21.51 & 22.21 & 0.88 & \\
\hline 141257.767 & +232618.93 & $*$ & 17.76 & 18.05 & 0.29 & 0.06 & 0.34 & 7.47 & 8.83 & 0.88 & \\
\hline 142134.300 & +421917.22 & $\mathrm{gE}$ & 15.17 & 16.85 & 1.67 & 0.03 & 0.57 & 2.11 & 2.06 & $\ldots$ & $\mathrm{O}(\mathrm{APS})$ \\
\hline 143655.700 & +234928.33 & $\mathrm{gO}$ & 17.62 & 19.26 & 1.64 & 0.09 & 0.95 & 1.86 & 2.02 & 0.15 & \\
\hline 144053.990 & +270642.23 & $*$ & 17.49 & 17.27 & -0.23 & 0.06 & 1.05 & 1.21 & 0.83 & 0.87 & \\
\hline 144755.745 & +382813.50 & $*$ & 17.79 & 19.06 & 1.27 & 0.03 & 0.73 & 1.18 & 2.28 & 0.11 & \\
\hline 145007.236 & +315050.77 & $*$ & 17.78 & 17.98 & 0.20 & 0.03 & 0.45 & 1.77 & 1.68 & 0.88 & \\
\hline 150228.568 & +354455.51 & $\mathrm{gO}$ & 17.12 & 19.01 & 1.90 & 0.04 & 0.26 & 2.50 & 2.63 & 0.11 & \\
\hline 150428.039 & +262419.54 & $*$ & 17.57 & 17.90 & 0.33 & 0.13 & 0.13 & 4.17 & 8.82 & 0.88 & \\
\hline 150435.882 & +335728.60 & $\mathrm{gO}$ & 17.76 & 19.39 & 1.63 & 0.04 & 0.28 & 1.58 & 1.18 & 0.11 & \\
\hline 150555.493 & +424415.86 & $*$ & 17.59 & 18.49 & 0.89 & 0.05 & 0.80 & 1.41 & 1.07 & 0.72 & \\
\hline 150624.223 & +322551.05 & $\mathrm{gE}$ & 14.11 & 15.09 & 0.99 & 0.05 & 0.21 & 3.39 & 3.21 & $\ldots$ & O(APS),I \\
\hline 151314.945 & +342111.86 & $*$ & 17.48 & 18.61 & 1.13 & 0.04 & 0.62 & 1.36 & 1.32 & 0.68 & \\
\hline 151627.376 & +305220.46 & $*$ & 17.52 & 19.05 & 1.53 & 0.05 & 0.62 & 1.08 & 0.87 & 0.63 & \\
\hline 151913.471 & +252134.24 & $*$ & 17.65 & 18.32 & 0.67 & 0.13 & 1.06 & 1.38 & 0.93 & 0.87 & \\
\hline 151958.370 & +352036.67 & $\mathrm{gE}$ & 15.20 & 16.13 & 0.93 & 0.05 & 0.89 & 2.10 & 2.51 & $\ldots$ & $\mathrm{O}(\mathrm{APS}), \mathrm{I}$ \\
\hline 152049.134 & +375219.18 & $*$ & 17.48 & 18.02 & 0.54 & 0.04 & 0.37 & 1.83 & 1.38 & 0.88 & \\
\hline 152149.082 & +373942.42 & $\mathrm{gO}$ & 17.51 & 18.50 & 0.99 & 0.04 & 0.64 & 1.64 & 1.68 & $\ldots$ & E(APS),O(APS),I \\
\hline 152158.455 & +3818 14.12 & $*$ & 17.65 & 18.71 & 1.06 & 0.04 & 1.12 & 1.17 & 1.30 & 0.61 & \\
\hline 152547.234 & +425210.75 & $*$ & 17.72 & 18.31 & 0.59 & 0.08 & 0.08 & 1.49 & 0.89 & 0.88 & \\
\hline 153402.247 & +425249.41 & $*$ & 17.74 & 18.28 & 0.54 & 0.07 & 0.18 & 8.33 & 8.54 & 0.88 & \\
\hline 153411.348 & +262124.53 & $\mathrm{gE}$ & 16.21 & 17.92 & 1.71 & 0.13 & 0.42 & 1.16 & 0.79 & 0.11 & \\
\hline 153420.266 & +413007.65 & $*$ & 17.36 & 19.26 & 1.91 & 0.07 & 0.40 & 5.36 & 5.39 & 0.42 & \\
\hline 153521.688 & +3318 26.48 & $*$ & 17.62 & 18.62 & 1.00 & 0.09 & 0.04 & 8.66 & 8.88 & 0.87 & \\
\hline 153818.661 & +410548.43 & $*$ & 17.51 & 17.88 & 0.36 & 0.07 & 0.86 & 26.03 & 50.37 & 0.87 & \\
\hline 154007.638 & +252836.79 & $\mathrm{gO}$ & 17.65 & 16.42 & -1.23 & 0.14 & 0.87 & 1.23 & 1.22 & 0.48 & \\
\hline 154049.233 & +390351.67 & $*$ & 17.59 & 18.16 & 0.57 & 0.06 & 0.72 & 1.03 & 1.61 & 0.86 & I \\
\hline 154433.216 & +322149.09 & $*$ & 17.69 & 17.79 & 0.09 & 0.08 & 0.19 & 11.39 & 12.02 & $\ldots$ & $\mathrm{E}(\mathrm{APS}), \mathrm{O}(\mathrm{APS})$ \\
\hline 155537.559 & +221327.11 & $\mathrm{gO}$ & 17.78 & 19.72 & 1.94 & 0.16 & 1.14 & 1.12 & 2.32 & 0.11 & \\
\hline 155723.917 & +420825.92 & $*$ & 17.64 & 18.44 & 0.80 & 0.05 & 0.52 & 1.16 & 0.70 & 0.87 & \\
\hline 160531.114 & +243147.59 & $*$ & 17.58 & 19.50 & 1.91 & 0.17 & 0.13 & 1.51 & 1.26 & 0.51 & \\
\hline 162237.898 & +235943.51 & $*$ & 17.76 & 19.51 & 1.75 & 0.17 & 0.96 & 1.02 & 2.80 & 0.11 & \\
\hline 162645.766 & +425709.38 & $*$ & 17.72 & 18.36 & 0.64 & 0.03 & 0.18 & 6.70 & 7.00 & $\cdots$ & $\mathrm{O}$ (APS) \\
\hline 163718.861 & +272607.71 & $*$ & 17.80 & 17.96 & 0.16 & 0.15 & 0.43 & 30.26 & 32.36 & 0.88 & \\
\hline 164733.911 & +364055.40 & $*$ & 17.77 & 18.56 & 0.79 & 0.07 & 0.66 & 1.12 & 0.75 & 0.87 & \\
\hline 170753.932 & +272418.64 & $*$ & 17.37 & 18.25 & 0.89 & 0.23 & 1.11 & 1.22 & 3.16 & 0.27 & \\
\hline
\end{tabular}

NotE.-Table columns are same as Table 7, with the addition of Col. $11 . P(Q)$ is the probability that the candidate is a quasar, computed using decision trees as described in the text. All source parameters including color were used. APS objects do not have values because the APS catalog does not have the APM PSF parameters used by the classifier. 
FIG. 16.- Spectra of FBQS candidates identified as quasars, sorted by decreasing redshift. The dotted lines show expected positions of prominent emission lines: Ly $\alpha$ 1216, N V 1240, Si IV 1400, C IV 1550, C III] 1909, Mg II 2800, H $\delta$ 4102, H $\gamma$ 4341, H $\beta$ 4862, [O III] 4959, [O III] 5007, H $\alpha$ 6563. Note that most of the spectra have atmospheric $\mathrm{A}$ and $\mathrm{B}$ band absorption at $\sim 6880 \mathrm{~A}$ and 7620 A. PLEASE SEE THE RELATED GIF FILES FOR THE ACTUAL SPECTRA. 
This figure "whiter16_01.gif" is available in "gif" format from: http://arxiv.org/ps/astro-ph/9912215v1 
This figure "whiter16_02.gif" is available in "gif" format from: http://arxiv.org/ps/astro-ph/9912215v1 
This figure "whiter16_03.gif" is available in "gif" format from: http://arxiv.org/ps/astro-ph/9912215v1 
This figure "whiter16_04.gif" is available in "gif" format from: http://arxiv.org/ps/astro-ph/9912215v1 
This figure "whiter16_05.gif" is available in "gif" format from: http://arxiv.org/ps/astro-ph/9912215v1 
This figure "whiter16_06.gif" is available in "gif" format from: http://arxiv.org/ps/astro-ph/9912215v1 
This figure "whiter16_07.gif" is available in "gif" format from: http://arxiv.org/ps/astro-ph/9912215v1 
This figure "whiter16_08.gif" is available in "gif" format from: http://arxiv.org/ps/astro-ph/9912215v1 
This figure "whiter16_09.gif" is available in "gif" format from: http://arxiv.org/ps/astro-ph/9912215v1 
This figure "whiter16_10.gif" is available in "gif" format from: http://arxiv.org/ps/astro-ph/9912215v1 
This figure "whiter16_11.gif" is available in "gif" format from: http://arxiv.org/ps/astro-ph/9912215v1 
This figure "whiter16_12.gif" is available in "gif" format from: http://arxiv.org/ps/astro-ph/9912215v1 
This figure "whiter16_13.gif" is available in "gif" format from: http://arxiv.org/ps/astro-ph/9912215v1 
This figure "whiter16_14.gif" is available in "gif" format from: http://arxiv.org/ps/astro-ph/9912215v1 
This figure "whiter16_15.gif" is available in "gif" format from: http://arxiv.org/ps/astro-ph/9912215v1 
This figure "whiter16_16.gif" is available in "gif" format from: http://arxiv.org/ps/astro-ph/9912215v1 
This figure "whiter16_17.gif" is available in "gif" format from: http://arxiv.org/ps/astro-ph/9912215v1 
This figure "whiter16_18.gif" is available in "gif" format from: http://arxiv.org/ps/astro-ph/9912215v1 
This figure "whiter16_19.gif" is available in "gif" format from: http://arxiv.org/ps/astro-ph/9912215v1 
This figure "whiter16_20.gif" is available in "gif" format from: http://arxiv.org/ps/astro-ph/9912215v1 
This figure "whiter16_21.gif" is available in "gif" format from: http://arxiv.org/ps/astro-ph/9912215v1 
This figure "whiter16_22.gif" is available in "gif" format from: http://arxiv.org/ps/astro-ph/9912215v1 
This figure "whiter16_23.gif" is available in "gif" format from: http://arxiv.org/ps/astro-ph/9912215v1 
This figure "whiter16_24.gif" is available in "gif" format from: http://arxiv.org/ps/astro-ph/9912215v1 
This figure "whiter16_25.gif" is available in "gif" format from: http://arxiv.org/ps/astro-ph/9912215v1 
This figure "whiter16_26.gif" is available in "gif" format from: http://arxiv.org/ps/astro-ph/9912215v1 
This figure "whiter16_27.gif" is available in "gif" format from: http://arxiv.org/ps/astro-ph/9912215v1 
This figure "whiter16_28.gif" is available in "gif" format from: http://arxiv.org/ps/astro-ph/9912215v1 
This figure "whiter16_29.gif" is available in "gif" format from: http://arxiv.org/ps/astro-ph/9912215v1 
This figure "whiter16_30.gif" is available in "gif" format from: http://arxiv.org/ps/astro-ph/9912215v1 
This figure "whiter16_31.gif" is available in "gif" format from: http://arxiv.org/ps/astro-ph/9912215v1 
This figure "whiter16_32.gif" is available in "gif" format from: http://arxiv.org/ps/astro-ph/9912215v1 
This figure "whiter16_33.gif" is available in "gif" format from: http://arxiv.org/ps/astro-ph/9912215v1 
This figure "whiter16_34.gif" is available in "gif" format from: http://arxiv.org/ps/astro-ph/9912215v1 
This figure "whiter16_35.gif" is available in "gif" format from: http://arxiv.org/ps/astro-ph/9912215v1 
This figure "whiter16_36.gif" is available in "gif" format from: http://arxiv.org/ps/astro-ph/9912215v1 
This figure "whiter16_37.gif" is available in "gif" format from: http://arxiv.org/ps/astro-ph/9912215v1 
This figure "whiter16_38.gif" is available in "gif" format from: http://arxiv.org/ps/astro-ph/9912215v1 
This figure "whiter16_39.gif" is available in "gif" format from: http://arxiv.org/ps/astro-ph/9912215v1 